\title{
Periodic Manifolds, Spectral Gaps, and Eigenvalues in Gaps
}

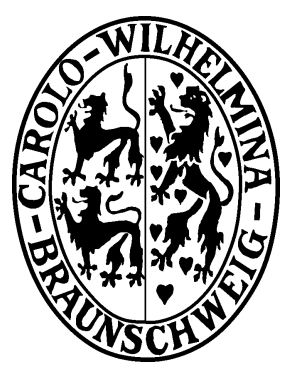

Vom Fachbereich für Mathematik und Informatik der Technischen Universität Braunschweig genehmigte

\author{
Dissertation \\ zur Erlangung des Grades eines \\ Doktors der Naturwissenschaften \\ (Dr. rer. nat.) \\ von \\ Olaf Post
}


Eingereicht am 30. Mai 2000

Datum der mündlichen Prüfung: 13. Juli 2000

1. Referent: Prof. Dr. Rainer Hempel

2. Referent: Priv.-Doz. Dr. Norbert Knarr 
Für Claudia 



\section{Contents}

$\begin{array}{ll}\text { Introduction } & 3\end{array}$

1 Preliminaries $\quad 11$

1.1 Hilbert spaces . . . . . . . . . . . . . . . . . . . 11

1.2 Operators and quadratic forms . . . . . . . . . . . . . . 11

1.3 Spectrum and Min-max Principle . . . . . . . . . . . . . . . . . . 13

1.4 Parameter-dependent Hilbert spaces . . . . . . . . . . . . . . . . . 15

1.5 Interchange of norm and quadratic form . . . . . . . . . . . . 20

2 Analysis on manifolds $\quad 23$

2.1 Spaces of square integrable functions . . . . . . . . . . . . . 23

2.2 Sobolev spaces . . . . . . . . . . . . . . . . . . 24

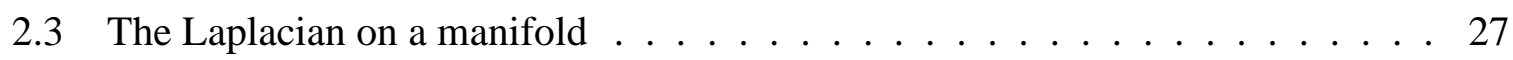

2.4 Metric perturbations . . . . . . . . . . . . . . . . 31

2.5 The Decomposition Principle . . . . . . . . . . . . . . . . 37

3 Floquet theory 39

3.1 Fourier analysis on abelian groups . . . . . . . . . . . . . . . . . . 39

3.2 Periodic manifolds and vector bundles . . . . . . . . . . . . . . . 39

3.3 Floquet decomposition . . . . . . . . . . . . . . . . 41

3.4 Periodic Laplacian on a manifold . . . . . . . . . . . . . . . . . . . . . . . . . . . . . . . . . . . .

3.5 Harmonic extension . . . . . . . . . . . . . . . . . . . . . . . . . . . . . . . . . . .

3.6 Periodic coverings . . . . . . . . . . . . . . . . . . . . . . . 49

4 Construction of a periodic manifold $\quad \mathbf{5 1}$

4.1 Metric estimates . . . . . . . . . . . . . . . . . . . 51

4.2 Construction of the period cell . . . . . . . . . . . . 53

4.3 Convergence of the eigenvalues . . . . . . . . . . . . . . . 54

4.4 Estimate on the cylindrical ends . . . . . . . . . . . . . . 56

5 Periodic manifold joined by cylinders 59

5.1 Construction of the period cell . . . . . . . . . . . . . . 59

5.2 Convergence of the eigenvalues . . . . . . . . . . . . . . . . 59

5.3 Estimate of the harmonic extension . . . . . . . . . . . . . 62 
6 Conformal deformation $\quad 65$

6.1 Conformal deformation . . . . . . . . . . . . . . . . . 65

6.2 Lower bounds for the eigenvalues . . . . . . . . . . . . . . . 67

6.3 Upper bounds for the eigenvalues . . . . . . . . . . . . . . . . . . . 69

7 The two-dimensional case $\quad 71$

7.1 What is different in the two-dimensional case? . . . . . . . . . . . . . . 71

7.2 Limit form in two dimensions . . . . . . . . . . . . . . . . . 72

7.3 Example . . . . . . . . . . . . . . . . . . 75

7.4 Mid-degree forms . . . . . . . . . . . . . . . . . . . . 79

8 Eigenvalues in spectral gaps $\quad 81$

8.1 Approximating problem . . . . . . . . . . . . . . . 81

8.2 Eigenvalue counting functions . . . . . . . . . . . . . 87

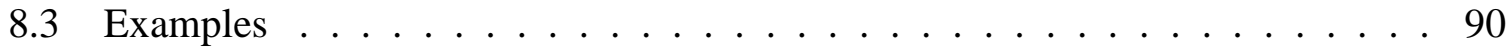

$\begin{array}{ll}\text { Bibliography } & 93\end{array}$

$\begin{array}{ll}\text { Zusammenfassung } & 97\end{array}$ 


\section{Introduction}

We investigate spectral properties of the Laplace operator on a class of non-compact Riemannian manifolds. We prove that for a given number $N$ we can construct a periodic manifold such that the essential spectrum of the corresponding Laplacian has at least $N$ open gaps. Furthermore, by perturbing the periodic metric of the manifold locally we can prove the existence of eigenvalues in a gap of the essential spectrum.

\section{Gaps in the spectrum}

In our context a periodic Riemannian manifold $M^{\text {per }}$ is a non-compact $d$-dimensional Riemannian manifold $(d \geq 2)$ with a properly discontinuous isometric action of an abelian group $\Gamma$ of infinite order such that the orbit space $M^{\text {per }} / \Gamma$ is compact. As in the case of periodic Schrödinger operators one can apply Floquet theory to show that the spectrum of the Laplacian $\Delta_{\mathbf{A}^{p} T M^{\text {per }}}$ on $M^{\text {per }}$ acting on $p$-forms (see Definition 2.3.1) has band structure, i.e., the spectrum $\operatorname{spec} \Delta_{\mathbf{A}^{p} T M^{\text {per }}}$ is the locally finite union of compact intervals $B_{k}\left(\Delta_{\mathbf{A}^{p} T M^{\mathrm{per}}}\right)$, called bands (see [Don81] if $\Gamma$ is abelian, [BS92] or [Gru98] for certain non-abelian groups $\Gamma$ or [RS78] in the Schrödinger operator case).

Here, we restrict ourselves to the Laplacian on functions, i.e., we suppose $p=0$. However, via the Hodge $*$-operator one can show that the spectrum of the Laplacian on functions is the same as the spectrum of the Laplacian on $d$-forms. Furthermore, supersymmetry in dimension 2 allows us to show that the spectrum of $\Delta_{\mathbf{A}^{p} T M^{\mathrm{per}}}$ is the same for $p=0, p=1$ and $p=2$. Therefore, all our results for the spectrum of the Laplacian on functions remain true in these special cases (see Theorems 2.3.8 and 3.4.6 and Corollaries 2.3.10 and 3.4.8).

In general, an infinite number of bands $B_{k}\left(\Delta_{\mathbf{A}^{p} T M^{\mathrm{per}}}\right)$ will overlap as in the case of the Laplacian $\Delta_{\mathbb{R}^{d}}=-\sum_{i=1}^{d} \partial_{i}^{2}$ on $\mathbb{R}^{d}$. Here, the spectrum is $[0, \infty[$.

Our first aim is to construct classes of (non-compact) periodic manifolds $M^{\text {per }}$ with gaps in the essential spectrum of the Laplacian $\Delta_{M^{\text {per }}}$ on $M^{\text {per }}$ acting on functions, i.e., we prove the existence of non-void intervals $] a, b[$ with

$$
\left.\operatorname{spec} \Delta_{M \operatorname{per}} \cap\right] a, b[=\emptyset .
$$

To exclude trivial cases we suppose that $a>\operatorname{infess} \operatorname{spec} \Delta_{M^{\text {per }}}$. Note that for (abelian-)periodic manifolds $M^{\text {per }}$ we always have infess $\operatorname{spec} \Delta_{M^{\text {per }}}=0$.

We prove the existence of gaps in two different ways. In both cases the main idea is to analyse a family of periodic manifolds $\left(M_{\varepsilon}^{\text {per }}\right)_{\varepsilon}$ such that $M_{\varepsilon}^{\text {per }}$ decouples in some sense as $\varepsilon \rightarrow 0$. By decoupling we mean that the junction between two period cells (see Section 3.2) is geometrically small. 


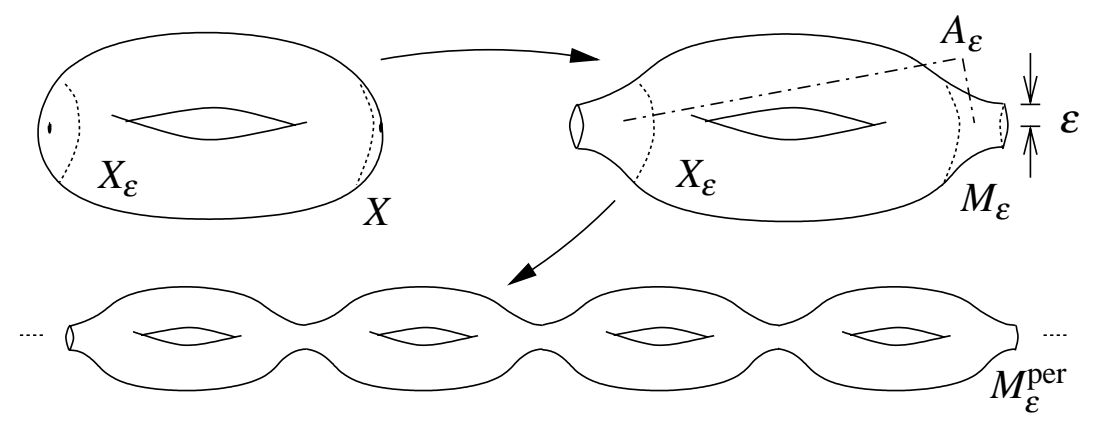

Figure 0.1: Construction of a periodic manifold in Case A.

Case A: We start with a compact $d$-dimensional Riemannian manifold $X$ (without boundary for simplicity). If $\Gamma=\mathbb{Z}$ we glue together $\mathbb{Z}$ copies of $X$ modified in the neighbourhood of two distinct points in such a way that we have two small cylindrical ends. The boundary of the modified manifold $M_{\varepsilon}$ is a $(d-1)$-dimensional sphere of radius $\varepsilon>0$ (see Figure 0.1 ). The resulting manifold $M_{\varepsilon}^{\text {per }}$ is $\mathbb{Z}$-periodic. Note that $M_{\varepsilon}^{\text {per }}$ still depends on $\varepsilon$. By Floquet theory, the analysis of the spectrum of $\Delta_{M_{\varepsilon}^{\text {per }}}$ is reduced to the analysis of the spectrum of the Laplacian on a period cell $M_{\varepsilon}$ with $\theta$-periodic boundary conditions where $\theta \in \hat{\mathbb{Z}}$ (see Section 3.4). The dual group $\hat{\Gamma}=\hat{\mathbb{Z}} \cong \mathbb{S}^{1}$ is usually identified with $[0,2 \pi[$ (see Section 3.1 ). Here, a period cell $M_{\varepsilon}$ is a closed subset of the periodic manifold $M_{\varepsilon}^{\text {per }}$ such that $M_{\varepsilon}^{\text {per }}$ is the union of all translates of $M_{\varepsilon}$ and such that $\stackrel{\circ}{M}_{\varepsilon}$ does not intersect any other translate of $M_{\varepsilon}$. Note that the spectrum of $\Delta_{M_{\varepsilon}}^{\theta}$ is discrete. We denote the eigenvalues written in increasing order by $\lambda_{k}^{\theta}\left(M_{\varepsilon}\right)$ counting multiplicities. In the same way, let $\lambda_{k}^{\theta}(X)$ denote the spectrum of the Laplacian $\Delta_{X}$ on $X$.

We prove the following (see Theorem 4.3.1 and Corollary 4.3.2):

Theorem. The $\theta$-periodic eigenvalues $\lambda_{k}^{\theta}\left(M_{\varepsilon}\right)$ converge uniformly in $\theta \in \hat{\mathbb{Z}} \cong \mathbb{S}^{1}$ to the eigenvalue $\lambda_{k}(X)$ as $\varepsilon \rightarrow 0$ for every $k \in \mathbb{N}$. In particular, if the $k$-th and the $(k+1)$-st eigenvalue of the Laplacian $\Delta_{X}$ on $X$ satisfy $\lambda_{k}(X)<\lambda_{k+1}(X)$, then there is a gap between the $k$-th and the $(k+1)$-st band of $\Delta_{M_{\varepsilon}^{\text {per }}}$, i.e.,

$$
B_{k}\left(\Delta_{M_{\varepsilon}^{\text {per }}}\right) \cap B_{k+1}\left(\Delta_{M_{\varepsilon}^{\text {per }}}\right)=\emptyset
$$

provided $\varepsilon$ is small enough.

Note that the convergence of the eigenvalue $\lambda_{k}^{\theta}\left(M_{\varepsilon}\right)$ is not uniform in $k$ (see page 8). Therefore we can prove that an arbitrary finite number of gaps occur if $\varepsilon$ is small enough. We can extend the theorem to the case of an arbitrary finitely generated abelian group $\Gamma$ (see Figure 0.2). We can also admit long thin cylinders of fixed length $L>0$ between the cylindrical ends as in Figure 0.3: The Laplacian of the resulting periodic manifold $\widetilde{M}_{\varepsilon}^{\text {per }}$ still has gaps if $\varepsilon$ is small enough (cf. Theorem 5.2.1 and Corrollary 5.2.2). This result was originally motivated by work of C. Anné (see [Ann87] and [Ann99]).

Case B: In the second class of examples, we start with a $\Gamma$-periodic Riemannian manifold $M^{\text {per }}$ (for simplicity) without boundary. We perturb the metric $g^{\text {per }}$ of $M^{\text {per }}$ conformally by a factor $\rho_{\varepsilon}^{2}$, i.e., we set $g_{\varepsilon}^{\text {per }}:=\rho_{\varepsilon}^{2} g^{\text {per }}$ and denote the resulting Riemannian manifold by $M_{\varepsilon}^{\text {per }}$. Here $\left(\rho_{\varepsilon}\right)$ is a family of strictly positive smooth periodic functions on $M^{\text {per }}$ converging pointwise to the indicator function of a set $X^{\text {per }}$. We suppose that $X^{\text {per }}$ is the disjoint union of the 


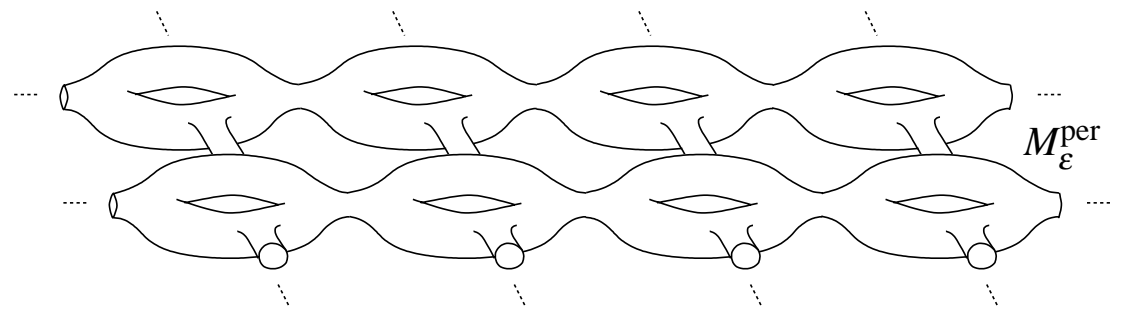

Figure 0.2: A manifold periodic with respect to a group generated by two elements (like $\mathbb{Z}^{2}$ or $\mathbb{Z} \times \mathbb{Z}_{p}$ ).

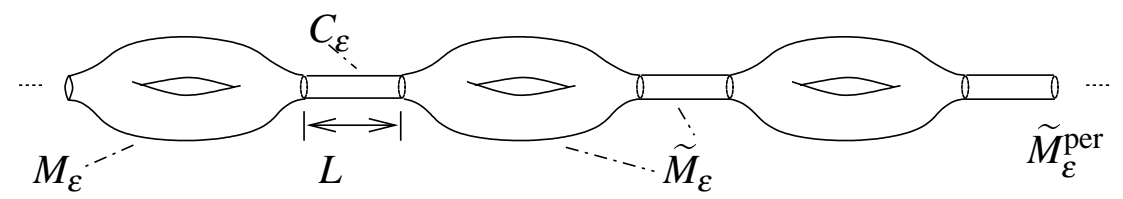

Figure 0.3: A periodic manifold with long thin cylinders obtained by taking $M_{\varepsilon}$ and $C_{\varepsilon}$ as new period cell $\widetilde{M}_{\varepsilon}$.

translates of a closed subset $X$ of $M^{\text {per }}$ such that there exists a period cell $M$ with $X \subset \stackrel{\circ}{M}$. Suppose further that normal coordinates with respect to $\partial X$ are defined on $\overline{M \backslash X}$ (see Section 6.1). This condition restricts the geometry of $X$. For example, a centered sphere in a cube as period cell satisfies this condition. Denote by $M_{\varepsilon}$ the manifold $M$ with metric $g_{\varepsilon}^{\text {per }}$ (see Figure 0.4 and Figure 0.5). Our second result is the following (see Theorem 6.1.2 and Corollary 6.1.3, for the Definition of the Neumann Laplacian $\Delta_{X}^{\mathrm{N}}$ see Definition 2.3.3):

Theorem. Suppose that $M^{\mathrm{per}}$ is of dimension $d \geq 3$. Then the $\theta$-periodic eigenvalues $\lambda_{k}^{\theta}\left(M_{\varepsilon}\right)$ converge uniformly in $\theta \in \hat{\Gamma}$ to the eigenvalue $\lambda_{k}^{\mathrm{N}}(X)$ of the Neumann Laplacian on $X$ as $\varepsilon \rightarrow 0$, for every $k \in \mathbb{N}$. In particular, if the $k$-th and the $(k+1)$-st eigenvalue of the Neumann Laplacian $\Delta_{X}^{\mathrm{N}}$ on $X$ satisfy $\lambda_{k}^{\mathrm{N}}(X)<\lambda_{k+1}^{\mathrm{N}}(X)$, then there is a gap between the $k$-th and the $(k+1)$-st band of $\Delta_{M_{\varepsilon}^{\text {per }}}$ provided $\varepsilon$ is small enough.

The two-dimensional case has to be treated separately. In this case we only prove that at least an arbitrary finite number of gaps exists if $M^{\text {per }}$ is a cylinder $\mathbb{R} \times \mathbb{S}^{1}$, see Chapter 7 .

The proof of the preceding two theorems is based on the Min-Max Principle (see Theorem 1.3.3). The main difficulty here comes from the fact that not only the quadratic form (corresponding to the Laplacian on $M_{\varepsilon}$ ) but also the $L_{2}$-norm on $M_{\varepsilon}$ depends on $\varepsilon$. We therefore compare the Rayleigh quotients for parameter-dependent Hilbert spaces (see Theorem 1.4.2). This idea is motivated by [Fuk87] and [Ann87], but we prove a slightly different version. One important ingredient in proving the preceding two theorems is a bound of the $L_{2}$-norm of eigenfunctions of $\Delta_{M_{\varepsilon}}^{\theta}$ on the cylindrical ends (Case A) resp. on $M \backslash X$ (Case B) converging to 0 as $\varepsilon \rightarrow 0$ (see Theorem 4.4.1 resp. Theorem 6.2.1, the estimates used there are motivated by [Ann94]).

In both cases gaps occur when there is a period cell $M_{\varepsilon}$ such that a neighbourhood of the boundary of $M_{\varepsilon}$ is small in some sense. Note that in Case A and Case B the volume of the $\varepsilon$-depending part $A_{\varepsilon}$ (resp. $A_{\varepsilon} \cup C_{\varepsilon}$ in the case of $\widetilde{M}_{\varepsilon}^{\text {per }}$ ) and $M_{\varepsilon} \backslash X$ converges to 0 . It seems that it is important to have a mechanism which "separates" or "decouples" in some sense the different translates of a period cell. 


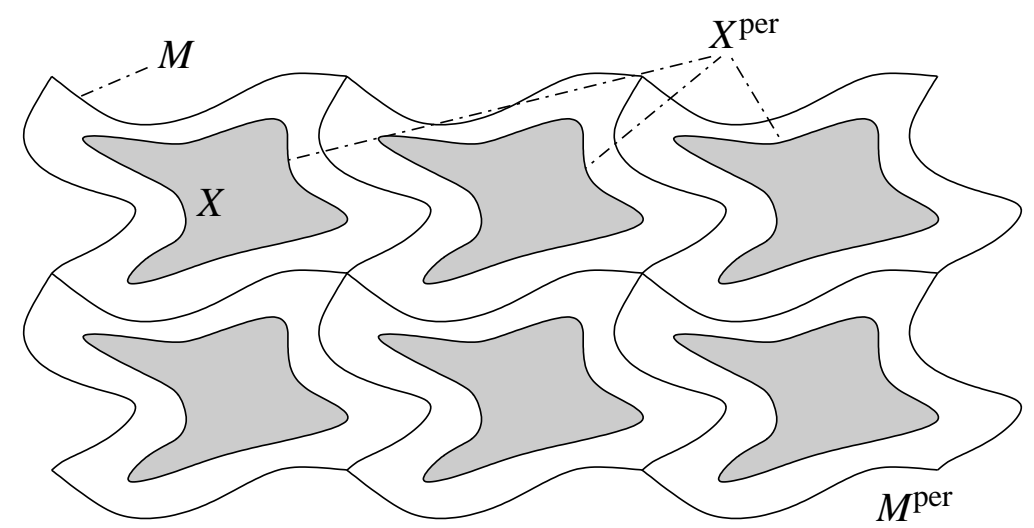

Figure 0.4: In Case B the $\mathbb{Z}^{2}$-periodic manifold $M^{\text {per }}$ is given. We choose a period cell $M$ such that the periodic subset $X^{\text {per }}$ does not intersect the boundary of $M$. We further suppose that normal coordinates with respect to $\partial X$ are defined on $\overline{M \backslash X}$.

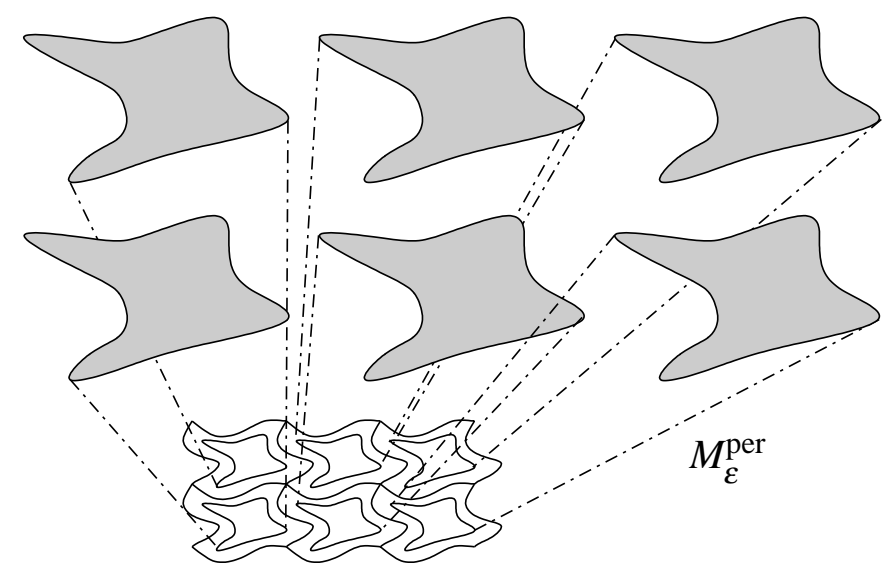

Figure 0.5: An, alas imperfect, attempt to picture the conformally perturbed manifold $M_{\varepsilon}^{\text {per }}$ obtained by scaling the manifold $M^{\text {per }}$ of Figure 0.4 outside the grey area $X^{\text {per }}$.

\section{Eigenvalues in gaps}

As an application of our results on spectral gaps, we perturb the metric of the periodic manifold $M^{\text {per }}$ locally and obtain eigenvalues in a gap of the periodic Laplacian. Suppose that $M^{\text {per }}=$ $M_{\varepsilon}^{\text {per }}$ is one of the periodic manifolds with period cell $M$ constructed before with metric $g^{\text {per }}$ such that $(*)$ holds. Since we will apply regularity theory we suppose that $\partial M$ is smooth (see e.g. the periodic manifold in Figure 0.2). Let $\lambda \in] a, b[$. If $\lambda$ is too close to $a$ or $b$ we possibly have to choose a smaller $\varepsilon$ (see Corollary 8.1.2). Suppose further that $(\rho(\tau))_{\tau>0}$ is a family of strictly positive smooth functions on $M^{\text {per }}$ such that $\rho(0)=1$ and such that $\rho(\tau)$ is equal to 1 outside a compact set $K_{1}$ and such that $\rho(\tau)$ is equal to $\tau+1$ on a compact set $K_{2} \subset \stackrel{\circ}{K}_{1}$ for all $\tau \geq 0$. Suppose further that $K_{1}$ and $K_{2}$ have (piecewise) smooth boundary and non-empty interior. We denote by $M(\tau)$ (resp. $K_{1}(\tau)$ and $\left.K_{2}(\tau)\right)$ the manifold $M^{\text {per }}\left(\right.$ resp. $K_{1}$ and $\left.K_{2}\right)$ with metric $g(\tau)=\rho(\tau)^{2} g^{\text {per }}$ conformal to $g^{\text {per }}$. Rougly speaking, we blow up the area $K_{1}$. In particular, the area $K_{2}$ is scaled by the factor $\tau+1 \geq 1$ (see Figure 0.6). 


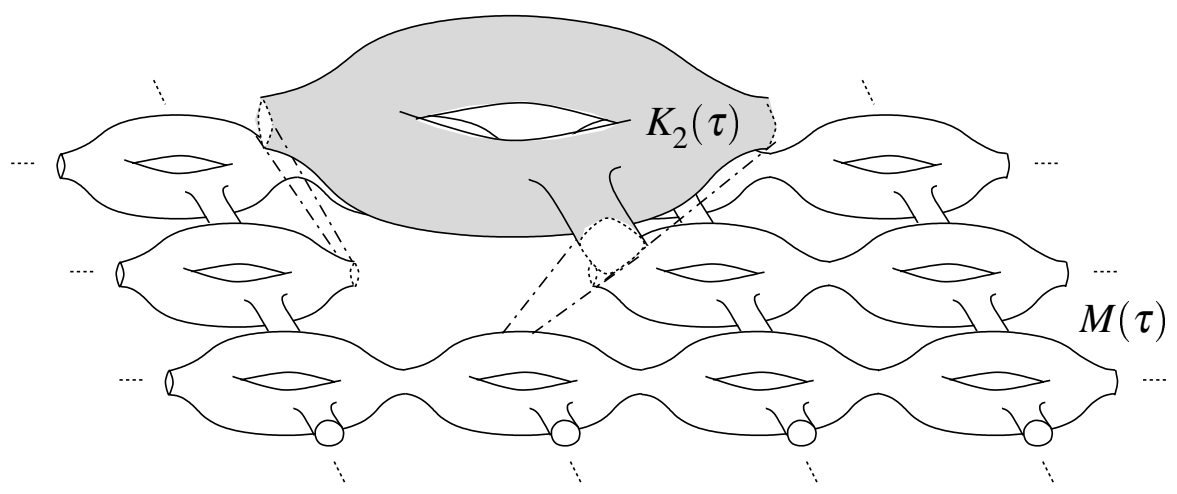

Figure 0.6: The perturbed manifold $M(\tau)$. Again, this picture is only an attempt since one cannot draw correctly the conformally perturbed area $K_{1}(\tau) \backslash K_{2}(\tau)$ where the conformal factor $\rho(\tau)$ is a nonconstant function.

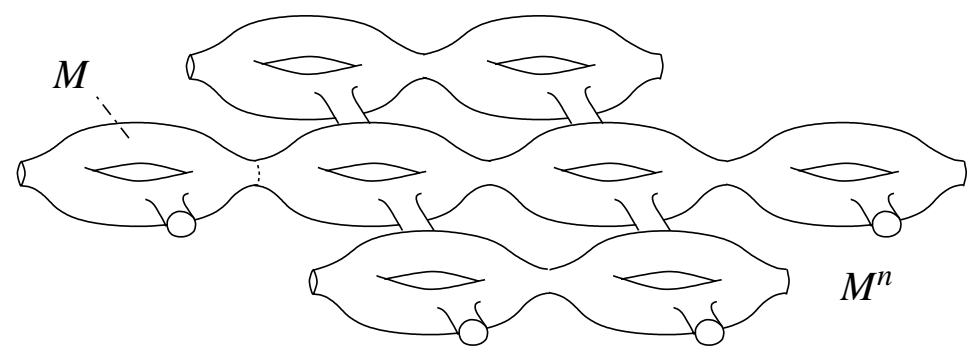

Figure 0.7: A periodic cell $M$ and an approximating submanifold $M^{n}$ of $M^{\text {per }}$ with $n=8$ elements.

By the Decomposition Principle (Theorem 2.5.1) the essential spectra of $\Delta_{M^{\text {per }}}$ and $\Delta_{M(\tau)}$ are the same, but in addition to the essential spectrum, $\Delta_{M(\tau)}$ could have discrete eigenvalues of finite multiplicity, accumulating only at the band edges of $\operatorname{spec} \Delta_{M^{\mathrm{per}}}$. Let

$$
\mathscr{N}_{\tau}\left(\Delta_{M(\cdot)}, \lambda\right):=\sum_{0 \leq \tau^{\prime} \leq \tau} \operatorname{dim} \operatorname{ker}\left(\Delta_{M\left(\tau^{\prime}\right)}-\lambda\right)
$$

denote the number of eigenvalues $\lambda$ (counted with respect to multiplicity) of the operator family $\left(\Delta_{M\left(\tau^{\prime}\right)}\right)_{0 \leq \tau^{\prime} \leq \tau}$. Among other results we prove the following (see Section 8.3.1):

Theorem. Let $] a, b\left[\right.$ be a gap in the spectrum of the unperturbed Laplacian $\Delta_{M^{\mathrm{per}}}$ and let $\lambda \in] a, b\left[\right.$. Then $\mathscr{N}_{\tau}\left(\Delta_{M(\cdot)}, \lambda\right)$ tends to infinity as $\tau \rightarrow \infty$. In particular, there exist $\tau>0$ such that $\lambda$ is an eigenvalue of the perturbed Laplacian $\Delta_{M(\tau)}$.

The idea is quite simple (cf. [AADH94] and [HB00]). We restrict ourselves to a compact approximating submanifold $M^{n}$ consisting of $n$ translates of a fixed period cell $M$ (see Figure 0.7). We further suppose that $M^{n}$ contains the perturbed domain $K_{1}$. Then the spectrum of the corresponding Dirichlet-Laplacian is purely discrete and we can count the eigenvalues arising from the perturbation by the Min-max principle. The main difficulty is to prove that eigenfunctions corresponding to a fixed eigenvalue $\lambda$ in a gap converge to eigenfunctions of the whole problem when $M^{n} \nearrow M^{\text {per }}$ (see Theorems 8.1.6 and 8.2.6). Again, we have to deal 
with the technical difficulty (which does not occur in [AADH94] or [HB00]) that our Hilbert spaces depend on the perturbation.

Note that the Dirichlet boundary condition on $M^{n}$ produces no eigenvalues in the gap ]$a+\eta, b-\eta$ [ (for some $\eta>0$ ) since the boundary of $M^{n}$ is small (see Figure 0.7 and Theorem 8.1.2). This fact simplifies our proof and we do not need the more complicated construction in [AADH94].

Note that we can express our results in the following way. Two metrics $g$ and $\widetilde{g}$ on $M^{\text {per }}$ are called conformally equivalent, if there is a strictly positive smooth function $\rho$ on $M^{\text {per }}$ such that $\widetilde{g}=\rho^{2} g$. A conformal structure given by $g$ is the equivalence class of $g$ (cf. [AG96]). Therefore we have proven:

Theorem. In every conformal structure on $M^{\text {per }}$ given by a periodic metric $g^{\text {per }}$ there are periodic metrics such that the corresponding Laplacian has at least an arbitrary finite number of gaps in its essential spectrum.

Furthermore there are (non-periodic) metrics $g$ in the conformal structure given by $g^{\text {per }}$ such that the spectrum of the Laplacian corresponding to $g$ has eigenvalues in a gap of its essential spectrum.

\section{Related results}

The question whether gaps exist in the spectrum of the Laplacian on a manifold and whether eigenvalues in gaps occur was motivated by similar results holding for divergence type operators (see [Hem92], [AADH94] or [HL99]). Note that, locally, the Laplacian on a manifold is a divergence type operator in a weighted $L_{2}$-space. As already mentioned, we cannot apply directly the existing results since the weight and therefore the Hilbert space depend on the metric which we want to perturb. Our work is also motivated by similar results in the case of Schrödinger operators; e.g., R. Hempel and I. Herbst have shown in [HH95a] and [HH95b] the existence of gaps in the spectrum of magnetic Schrödinger operators.

E. B. Davies and E. M. Harrell II proved in [DH87] the existence of at least one gap in the spectrum of the Laplacian defined by a conformally flat periodic metric on $\mathbb{R}^{d}(d \geq 2)$, i.e., $g_{i j}=\rho^{2} \delta_{i j}$ where $\rho$ is a strictly positive smooth function on $\mathbb{R}^{d}$. Here, a relation between the conformally flat Laplacian and certain Schrödinger operators is established. Furthermore, E. L. Green proved in [Gre97] the existence of a finite number of gaps of a conformally flat Laplacian with periodic metric on $\mathbb{R}^{2}$.

Recently, Lott proved the following result in [Lot99, Theorem 3]. For every $\varepsilon>0$ there exists a complete connected non-compact finite-volume Riemannian manifold whose sectional curvatures lie in $[-1-\varepsilon,-1+\varepsilon]$ and whose Laplacian on functions has an infinite number of gaps in its spectrum. The gaps tend towards infinity. He starts with a complete finite-volume hyperbolic metric on a punctured 2-torus and changes the metric on the cusp.

A more general context is given in [BS92]. The authors establish an asymptotic upper bound on the number of gaps in the spectrum of a $\Gamma$-periodic elliptic semi-bounded operator. Here, even certain non-abelian groups $\Gamma$ are admitted.

Note that the convergence of the eigenvalue $\lambda_{k}^{\theta}\left(M_{\varepsilon}\right)$ in Case A and B is not uniform in $k$ : there are topological obstructions that prevent the uniform convergence (cf. [CF81]). This result is in accordance with the general Bethe-Sommerfeld conjecture which says that the spectrum of any $\Gamma$-periodic Schrödinger operator $\Delta_{M^{\text {per }}}+V$ with potential $V$ on a periodic manifold 
$M^{\text {per }}$ of dimension $d \geq 2$ has only a finite number of gaps, i.e., the intersection of the resolvent set with $\mathbb{R}$ has only a finite number of components. Note that if $\lambda_{k}^{\theta}\left(M_{\varepsilon}\right)$ would converge uniformly in $\theta \in \hat{\Gamma}$ and $k \in \mathbb{N}$ then there would be an infinite number of gaps in $\operatorname{spec} \Delta_{M_{\varepsilon}^{\text {per }}}$ if $\varepsilon$ is small enough.

In [SY94, Open Problem 37], Yau proposed to analyse the spectrum of the Laplacian acting on differential forms of a non-compact manifold. In particular, he posed the question whether the continuous spectrum of the Laplacian has band structure. It is well-known that the answer is yes on a periodic manifold (by using Floquet Theory, see Chapter 3). He further asked about the stability of the continuous spectrum when changing the metric uniformly. In Chapter 8 , we prove that the essential spectrum is stable provided the perturbation is small outside a compact set by using the well-known decomposition principle (see Theorem 8.1.7).

\section{Applications}

In the theory of continuum mechanics Laplace operators on manifolds can be used to give the necessary equations for dilational waves on a surface (see [Jau67, pp. 341-346] or [Jau72]).

Another application is connected with the recent development of the theory of quantum wave guides or quantum wires (cf. [EŠ89, pp. 257-266] and [LCM99]). Here, a quantum wire is a two-dimensional curved planar strip $\Omega$ of a fixed width $d$. If $d$ is small, the DirichletLaplacian on $\Omega$ describes the motion of a free electron living on $\Omega$. Such models could be appropriated for microelectronics. Since the scales become smaller and smaller one should also consider quantum effects. Here, a quantum wire is a thin layer of shape $\Omega$ in a semiconductor. Yoshitomi proved the existence of gaps in the spectrum of the (flat) Laplacian with Dirichlet boundary conditions on a periodically curved strip in $\mathbb{R}^{2}$ (see [Yos98]).

Our results show the same behaviour more generally for certain periodically curved surfaces, i.e., periodic manifolds of dimension 2. This could model very thin non-planar periodic layers made out of metallic or semi-conducting materials. Therefore we have proven the existence of gaps in the energy spectrum of an electron moving along this layer. Note that the band-gap structure and eigenvalues in gaps are important for the conductor properties of the material. In particular, local perturbation of a periodic surface leads to impurity levels in an energy gap (i.e., eigenvalues in the gap).

\section{Outlook}

In the proof of the existence of eigenvalues in a gap of the unperturbed Laplacian (see Chapter 8) we have assumed that the periodic manifold $M^{\text {per }}$ possesses a period cell $M$ with smooth boundary. This is necessary since we want to apply regularity theory on a domain given by (the union of translates of) $M$. In particular, the case $M^{\text {per }}=\mathbb{R}^{d}$ is excluded, since any period cell has singularities. Suppose that the group $\Gamma$ with $r$ generators acts on $M^{\text {per }}$ properly discontinuously and cocompactly. What kind of singularities could appear if one chooses connected period cells with piecewise smooth boundary? Furthermore, can one choose a period cell $M$ with "tame" singularities such that we still can apply regularity theory? A positive answer to these questions would give further examples of eigenvalues in a gap. 
It is an interesting question wether our results extend also to the non-ablian case, i.e., whether there exists a $\Gamma$-periodic manifold with a non-abelian group $\Gamma$ such that the corresponding Laplacian has gaps. Note that such manifolds are of interest since for any Riemannian manifold $M^{\text {per }}$ with strictly negative sectional curvature, $\mathbb{Z}$ is the maximal abelian subgroup of any group $\Gamma$ acting isometrically and properly discontinuously on $M^{\text {per }}$ by a theorem of Preissmann (see [Pre42, Theorème $7 *]$ or [Bye70]). Therefore if we only allow abelian groups $\Gamma$ with more than one generator our results (e.g. on the existence of gaps in the spectrum of the Laplacian) do not apply to the case of (non-compact) Riemannian manifolds with strictly negative curvature (i.e., hyperbolic spaces).

Furthermore, we have not proved the existence of gaps in the spectrum of the Laplacian $\Delta_{\mathbf{A}^{p} T M \text { per }}$ acting on $p$-forms. Here, our methods do not directly apply exept for the case of $d$-forms or for dimension 2 .

\section{An Overview of the Text}

In Chapter 1 we quote some basic facts on Hilbert spaces and Spectral Theory. We also develop some results on parameter-dependent Hilbert spaces (see Section 1.4). In Chapter 2 we give the necessary background to define the Laplacian on a manifold. Furthermore, in Section 2.4 we analyse how several estimates depend on the metric. We give quite explicit proofs here since we need control of the constants. In Section 2.5 we quote the decomposition principle, i.e., the stability of the essential spectrum under local perturbations. Chapter 3 is devoted to Floquet Theory. Here, we define the term "periodic" for manifolds. In Chapters 4 and 5 we construct a first class of periodic manifolds such that the corresponding Laplacians have gaps in the essential spectrum (see Case A). In Chapters 6 and 7 we produce gaps by a conformal periodic perturbation of a given metric (see Case B). Finally, in Chapter 8 we prove the existence of eigenvalues in a spectral gap obtained by a local perturbation of the metric.

\section{Acknowledgements}

I am indebted to my thesis advisor R. Hempel for his kind attention and innumerable discussions. I would like to thank N. Knarr for his willingness to be the co-referee of this work. I would also like to thank I. Chavel for drawing our attention to an article of C. Anné, C. Anné for the helpful discussion concerning her article and C. Bär, W. Ballmann and G. Carron for making useful suggestions on Section 7.4. 


\section{Preliminaries}

In this chapter we introduce the necessary Hilbert space notation and some related results. Most of the statements are standard, see e. g. [Kat66], [RS80] or [Dav96].

\subsection{Hilbert spaces}

Let $\mathscr{H}$ be a complex, separable Hilbert space with inner product $\langle\cdot, \cdot\rangle_{\mathscr{H}}$. We always suppose that the inner product (or any other sesquilinear form) is linear in the first and antilinear in the second argument. The norm induced by the inner product is denoted by $\|u\|_{\mathscr{H}}=\|u\|:=$ $\left(\langle u, u\rangle_{\mathscr{C}}\right)^{\frac{1}{2}}$. It is well-known that bounded subsets of a Hilbert space are sequentially compact in the weak topology:

1.1.1. Theorem. Let $\left(u_{m}\right)_{m}$ be a bounded sequence in the Hilbert space $\mathscr{H}$. Then we can find a subsequence $\left(u_{m_{n}}\right)_{n}$ such that $u_{m_{n}}$ converges to an element $u \in \mathscr{H}$ in the weak topology, i.e.,

$$
\left\langle u_{m_{n}}, v\right\rangle_{\mathscr{H}} \rightarrow\langle u, v\rangle_{\mathscr{H}}, \quad n \rightarrow \infty
$$

for all $v \in \mathscr{H}$.

Note that norm-convergence of a sequence of vectors in a Hilbert space is sometimes called strong convergence.

A simple consequence of the Cauchy-Schwarz Inequality is the Cauchy-Young Inequality

$$
\left|\langle u, v\rangle_{\mathscr{H}}\right| \leq\|u\|_{\mathscr{H}}^{2}+\|v\|_{\mathscr{H}}^{2} \text {. }
$$

\subsection{Operators and quadratic forms}

We briefly give some facts on (unbounded) operators in Hilbert spaces. Suppose that $\mathscr{H}, \mathscr{H}_{1}$ and $\mathscr{H}_{2}$ are separable Hilbert spaces.

A linear operator $Q: \operatorname{dom} Q \longrightarrow \mathscr{H}_{2}$ on a linear subspace $\operatorname{dom} Q$ of $\mathscr{H}_{1}$ is called densely defined if $\operatorname{dom} Q$ is dense in $\mathscr{H}_{1}$. The operator $Q$ is called closed if its graph in $\mathscr{H}_{1} \times \mathscr{H}_{2}$ is a closed subspace.

For a densely defined operator $Q: \mathscr{H}_{1} \longrightarrow \mathscr{H}_{2}$ we let $\operatorname{dom} Q^{*}$ be the set of $v \in \mathscr{H}_{2}$ such that the map

$$
\langle Q \cdot v\rangle_{\mathscr{H}_{2}}: \operatorname{dom} Q \longrightarrow \mathbb{C}, \quad u \longmapsto\langle Q u, v\rangle_{\mathscr{H}_{2}}
$$

is continuous. Therefore $\operatorname{dom} Q^{*}$ is a linear subspace of $\mathscr{H}_{2}$ and for $v \in \operatorname{dom} Q^{*}$ the map $u \mapsto\langle Q u, v\rangle_{\mathscr{H}_{2}}$ can be extended uniquely to a continuous linear functional on $\mathscr{H}_{1}$. By the 
representation theorem of Riesz, this functional can be written as $\langle\cdot, w\rangle_{\mathscr{H}_{1}}$ for precisely one $w \in \mathscr{H}_{1}$. We set $Q^{*} v:=w$ and we obtain

$$
\langle Q u, v\rangle_{\mathscr{H}_{2}}=\left\langle u, Q^{*} v\right\rangle_{\mathscr{H}_{1}}
$$

for all $u \in \operatorname{dom} Q$ and $v \in \operatorname{dom} Q^{*}$.

We can prove the following result by using the adjoint operator:

1.2.1. Lemma. A bounded (i.e., norm-continuous) operator $Q: \mathscr{H}_{1} \longrightarrow \mathscr{H}_{2}$ is also weakly continuous, i.e., $u_{n} \rightarrow 0$ weakly in $\mathscr{H}_{1}$ implies $Q u_{n} \rightarrow 0$ weakly in $\mathscr{H}_{2}$.

Proof. We have $\left\langle Q u_{n}, v\right\rangle_{\mathscr{H}_{2}}=\left\langle u_{n}, Q^{*} v\right\rangle_{\mathscr{H}_{1}} \rightarrow 0$ for all $v \in \mathscr{H}_{2}$.

If $\mathscr{H}:=\mathscr{H}_{1}=\mathscr{H}_{2}$, an operator $Q$ is called symmetric if $Q$ is densely defined and $\langle Q u, v\rangle_{\mathscr{H}}=\langle u, Q v\rangle_{\mathscr{H}}$ holds for all $u, v \in \operatorname{dom} Q$. The symmetric operator $Q$ is called positive $(Q \geq 0)$ if $\langle Q u, u\rangle_{\mathscr{H}} \geq 0$ for all $u \in \operatorname{dom} Q$. A densely defined operator $Q$ is called self-adjoint if $Q=Q^{*}$, i.e., $\operatorname{dom} Q=\operatorname{dom} Q^{*}$ and $Q u=Q^{*} u$ for all $u \in \operatorname{dom} Q$. Self-adjoint operators are symmetric, the converse is not true in general (for unbounded operators).

A sesquilinear map $q: \operatorname{dom} q \times \operatorname{dom} q \longrightarrow \mathbb{C}$ (linear in the first, antilinear in the second argument) is called densely defined if $\operatorname{dom} q$ is dense in $\mathscr{H}$. We often call a sesquilinear map a (sesquilinear) form.

A sesquilinear form $q$ is called symmetric if $q(u, v)=\overline{q(v, u)}$ or, equivalently, $q(u, u) \in \mathbb{R}$ is satisfied for all $u, v \in \operatorname{dom} q$. It is called positive if $q(u) \geq 0$ for all $u \in \operatorname{dom} q$.

A positive form $q$ is called closed if $\operatorname{dom} q$ equipped with the inner product

$$
\langle u, v\rangle_{q}:=\langle u, v\rangle_{\mathscr{H}}+q(u, v)
$$

is complete, therefore itself a Hilbert space. We call the corresponding norm q-norm or form norm (of $q$ ). If we speak of topological properties of $\operatorname{dom} q$ we always mean the corresponding topology arising from (1.2), e.g., a $q$-norm-dense subspace $\mathscr{D}$ in $\operatorname{dom} q$ is called a form core. If $q$ is positive and densely defined, we can always construct the (unique) smallest closed extension $\bar{q}$, called the closure of $q$. By construction, $\operatorname{dom} q$ is a form core of $\operatorname{dom} \bar{q}$. In the sequel we do not distinguish between $q$ and $\bar{q}$.

By the Polarisation Identity

$$
q(u, v)=\frac{1}{4}(q(u+v)-q(u-v)+\mathrm{i} q(u+\mathrm{i} v)-\mathrm{i} q(u-\mathrm{i} v))=\frac{1}{4} \sum_{n=0}^{3} \mathrm{i}^{n} q\left(u+\mathrm{i}^{n} v\right)
$$

every sesquilinear form is determined by its (quadratic) form $q(u):=q(u, u)$.

The following representation theorem (see [Kat66, theorem VI.2.1]) associates to every positive (densely defined) form $q$ a unique self-adjoint operator $Q$ :

1.2.2. Theorem. For every densely defined closed positive sesquilinear form $q$ there exists precisely one positive self-adjoint operator $Q$ such that $\operatorname{dom} Q \subset \operatorname{dom} q$ and

$$
q(u, v)=\langle Q u, v\rangle_{\mathscr{H}}
$$

for all $u \in \operatorname{dom} Q$ and $v \in \operatorname{dom} q$. The domain $\operatorname{dom} Q$ of $Q$ consists of $u \in \operatorname{dom} q$ such that

$$
q(u, \cdot): \operatorname{dom} q \longrightarrow \mathbb{C}, \quad v \longmapsto q(u, v)
$$

is norm-continuous. Furthermore, $\operatorname{dom} Q$ is a form core. 
As in the case of the adjoint operator, we have the following characterization of the operator domain: $\operatorname{dom} Q$ consists of exactly those $u \in \operatorname{dom} q$ for which there exists a (unique) element $w=: Q u$ such that (1.4) is satisfied for all $v \in \operatorname{dom} q$. It is sufficient to prove (1.4) for all $v$ in a form core.

We often deal rather with the quadratic form than with the operator since it is much easier to deal with the form domain $\operatorname{dom} q$ than the operator $\operatorname{domain} \operatorname{dom} Q$. Note that $\operatorname{dom} q=\operatorname{dom} Q^{\frac{1}{2}}$.

In the sequel, we will assume without mentioning that our forms and operators are densely defined.

\subsection{Spectrum and Min-max Principle}

We define the spectrum $\operatorname{spec} Q$ of a closed operator $Q$ as the set of those $\lambda \in \mathbb{C}$ for which $Q-\lambda$ does not have a bounded inverse. From now on we suppose that all our operators are self-adjoint. In particular, if $Q$ is self-adjoint, then $\operatorname{spec} Q \subset \mathbb{R}$.

The discrete spectrum of a self-adjoint operator $Q$ consists of the eigenvalues $\lambda$ of finite multiplicity which are isolated in the sense that $] \lambda-\varepsilon, \lambda[$ and $] \lambda, \lambda+\varepsilon[$ are disjoint from $\operatorname{spec} Q$ for some $\varepsilon>0$. The essential spectrum ess $\operatorname{spec} Q$ is the non-discrete part of $\operatorname{spec} Q$. We have the following characterisation of the essential spectrum:

1.3.1. Lemma (Weyl's criterion). Let $Q$ be a self-adjoint operator. Then $\lambda \in \operatorname{ess} \operatorname{spec} Q$ if and only if there exists a sequence $\left(u_{n}\right) \subset \operatorname{dom} Q$ such that $\left\|u_{n}\right\|=1, u_{n} \rightarrow 0$ weakly and $\left\|(Q-\lambda) u_{n}\right\| \rightarrow 0$ as $n \rightarrow \infty$.

The sequence $\left(u_{n}\right)$ is called a singular sequence for $\lambda$ and $Q$.

An operator $Q$ has purely discrete spectrum if ess $\operatorname{spec} Q=\emptyset$. From now on, we denote the eigenvalues of a positive operator with purely discrete spectrum by $\lambda_{k}$ or $\lambda_{k}(Q)$ (or similar notations) written in increasing order and repeated according to multiplicity. Thus the eigenvalues of such an operator satisfy $\lim _{k \rightarrow \infty} \lambda_{k}=\infty$.

An operator $K: \mathscr{H}_{1} \longrightarrow \mathscr{H}_{2}$ is compact if $\left(K u_{n}\right)$ converges to 0 in norm for any sequence $\left(u_{n}\right)$ converging weakly to 0 . If $\mathscr{H}=\mathscr{H}_{1}=\mathscr{H}_{2}$ and $K \geq 0$ then this is equivalent to the fact that spec $K \backslash\{0\}$ consists only of discrete points. These points are eigenvalues of finite multiplicity converging to 0 if they are ordered in decreasing order and repeated according to multiplicity. Note that $(Q+1)^{-1}$ is compact if and only if $Q \geq 0$ has purely discrete spectrum. We have another criterion:

1.3.2. Lemma. Let $q$ be a positive form with corresponding operator $Q$. Then $(Q+1)^{-1}$ is compact if and only if the embedding map

$$
\imath:\left(\operatorname{dom} q,\|\cdot\|_{q}\right) \hookrightarrow\left(\mathscr{H},\|\cdot\|_{\mathscr{H}}\right)
$$

is compact.

The Min-max Principle allows us to obtain quantitative estimates of eigenvalues and to compare the eigenvalues of different operators. Let $Q$ be a positive self-adjoint operator with corresponding (densely defined, closed, positive) form $q$. Suppose further that $Q$ has purely discrete spectrum. Let $L$ be any finite-dimensional subspace of dom $q$. We define

$$
\lambda_{L}(q):=\sup \left\{\frac{q(u)}{\|u\|^{2}} \mid u \in L, u \neq 0\right\} .
$$


The quotient $q(u) /\|u\|^{2}$ is called Raleigh-quotient of $q$. The following theorem is called Minmax Principle:

1.3.3. Theorem. The eigenvalues $\lambda_{k}(Q)$ of a positive self-adjoint operator $Q$ with corresponding form q satisfy

$$
\lambda_{k}(Q)=\inf \left\{\lambda_{L}(q) \mid L \subset \operatorname{dom} q \text { and } \operatorname{dim} L=k\right\} .
$$

1.3.4. Definition. Let $q_{1}$ resp. $q_{2}$ be a sesquilinear form with domain dom $q_{1}$ resp. $\operatorname{dom} q_{2}$. We say that $q_{1}$ is smaller or equal than $q_{2}\left(q_{1} \leq q_{2}\right)$ iff

$$
\operatorname{dom} q_{1} \supset \operatorname{dom} q_{2} \quad \text { and } \quad q_{1}(u) \leq q_{2}(u)
$$

for all $u \in \operatorname{dom} q_{2}$.

Note that the inclusion of the domains in (1.6) is motivated by setting $q_{i}(u):=\infty$ for all $u \notin \operatorname{dom} q_{i}$ for $i=1$ or $i=2$. Then we have $q_{1} \leq q_{2}$ if and only if $q_{1}(u) \leq q_{2}(u)$ for all $u \in \mathscr{H}$. With this convention we can allow any $k$-dimensional subspace $L \subset \mathscr{H}$ in (1.5).

Since the Min-max Principle for an operator with purely discrete spectrum describes the $k$-th eigenvalue only in terms of the quadratic form and its domain, we have the following corollary of the Min-max Principle:

1.3.5. Corollary. Let $q_{1}$ and $q_{2}$ be closed positive forms with corresponding operators $Q_{1}$ and $Q_{2}$. Suppose that $Q_{1}$ has purely discrete spectrum. Furthermore suppose $q_{1} \leq q_{2}$ or, in particular, that $q_{2}$ is the restriction of $q_{1}$ to the subspace $\operatorname{dom} q_{2}$ of $\operatorname{dom} q_{1}$. Then $Q_{2}$ has purely discrete spectrum and we have $\lambda_{k}\left(Q_{1}\right) \leq \lambda_{k}\left(Q_{2}\right)$.

We will make frequent use of this corollary since in many of our applications the forms will only differ in their domain, not in their formal expression.

Next, we define the eigenvalue counting function:

1.3.6. Definition. Suppose $Q \geq 0$ is a self-adjoint operator in $\mathscr{H}$. We set

$$
\operatorname{dim}_{I}(Q):=\operatorname{dim} \operatorname{Ran} E_{I}(Q)
$$

where $E_{I}(Q)$ denotes the spectral projection of $Q$ on the measurable set $I \subset \mathbb{R}_{+}=[0, \infty[$. If furthermore $Q$ has discrete spectrum then

$$
\operatorname{dim}_{I}(Q)=\sum_{\lambda^{\prime} \in I} \operatorname{dim} \operatorname{ker}\left(Q-\lambda^{\prime}\right)=\operatorname{card}\left\{k \in \mathbb{N} \mid \lambda_{k}(Q) \in I\right\}
$$

denotes the number of eigenvalues lying in $I$. In particular, $\operatorname{dim}_{\lambda}(Q):=\operatorname{dim}_{[0, \lambda]}(Q)$ denotes the number of eigenvalues $\lambda_{k}(Q)$ of $Q$ not greater than $\lambda$.

The following simple statements will be needed in Chapter 8. More details can be found in [RS78, Section XIII.15].

1.3.7. Lemma. Let $Q_{1}, Q_{2} \geq 0$ be operators with purely discrete spectrum.

1. If $Q_{1} \leq Q_{2}$ then $\operatorname{dim}_{\lambda}\left(Q_{1}\right) \geq \operatorname{dim}_{\lambda}\left(Q_{2}\right)$. 
2. Suppose $\mathscr{H}=\mathscr{H}_{1} \oplus \mathscr{H}_{2}$ and $Q_{i}\left(\mathscr{H}_{i}\right) \subset \mathscr{H}_{i}$ for $i=1$ and $i=2$. Then $\operatorname{dim}_{\lambda}\left(Q_{1} \oplus Q_{2}\right)=$ $\operatorname{dim}_{\lambda}\left(Q_{1}\right)+\operatorname{dim}_{\lambda}\left(Q_{2}\right)$.

Finally, we sketch the idea of supersymmetry to obtain spectral information (see [CFKS87, Section 6.3] or [BGV92, Section 1.3]). Let $H$ and $Q$ be self-adjoint operators and let $P$ be a self-adjoint, bounded operator on the Hilbert space $\mathscr{H}$.

1.3.8. Definition. The triple $(H, P, Q)$ has supersymmetry, if

$$
H=Q^{2} \geq 0, \quad P^{2}=1, \quad \text { and } \quad\{P, Q\}:=P Q+Q P=0 .
$$

From the definition we conclude:

1.3.9. Lemma. Denote by $\mathscr{H}_{ \pm}$the eigenspace corresponding to the eigenvalue \pm 1 of $P$. Then $\mathscr{H}=\mathscr{H}_{+} \oplus \mathscr{H}_{-}$. Furthermore, $Q_{ \pm}:=Q \uparrow_{\mathscr{H}_{ \pm}}$maps (elements of a subset of) $\mathscr{H}_{ \pm}$into $\mathscr{H}_{\mp}$ and $H=Q_{-} Q_{+} \oplus Q_{+} Q_{-}$.

Proof. Clearly, $\operatorname{spec} P \subset\{ \pm 1\}$. Let $u \in \operatorname{dom} Q \subset \mathscr{H}_{ \pm}$. Then $Q u= \pm Q P u=\mp P Q u$, i.e., $Q u \in \mathscr{H}_{\mp}$. Furthermore $H u=Q_{\mp} Q_{ \pm} u$.

The following theorem shows that supersymmetry is useful to analyse the spectrum of $H$.

1.3.10. Theorem. We have $\operatorname{dim}_{I}\left(H_{+}\right)=\operatorname{dim}_{I}\left(H_{-}\right)$for all measurable sets $\left.I \subset\right] 0, \infty[$. In particular, $\operatorname{spec} H_{+} \backslash\{0\}=\operatorname{spec} H_{-} \backslash\{0\}$.

Proof. For a detailed proof see [CFKS87, Theorem 6.3]. If $H$ has purely discrete spectrum, then $H_{ \pm} u=\lambda u$ implies $H_{\mp} Q_{ \pm} u=Q_{ \pm} H_{ \pm} u=\lambda Q_{ \pm} u$. Since $\lambda \neq 0$, it follows that $u \notin \operatorname{ker} H$ and therefore $u \notin \operatorname{ker} Q$, i.e., $Q_{ \pm} u \neq 0$ if and only if $u \neq 0$.

\subsection{Parameter-dependent Hilbert spaces}

Since we will consider $L_{2}$-spaces on Riemannian manifolds with metrics $g_{\varepsilon}$ depending on a parameter $\varepsilon$, all Hilbert spaces will depend on $\varepsilon$. In most cases only the inner product and thus the norm will depend on $\varepsilon$ while the vector space structure will be the same. We nevertheless prefer to discuss the general context even if it is quite technical.

To obtain convergence of the corresponding eigenvalues of the Laplacian we need several estimates for the parameter-dependent eigenvalues, each of the Laplacians acting in a different Hilbert space. We formulate the result in an abstract Hilbert space framework. Our Theorem 1.4.2 is influenced by [Ann87, Théorème 1], [Ann99] and [Fuk87, Section 5], but we prove a slightly different version of Lemma 5.1 in [Fuk87].

We assume that $\mathscr{H}_{\varepsilon}$ and $\mathscr{H}_{\varepsilon}^{\prime}$ are separable Hibert spaces for each $\varepsilon>0$. Furthermore, we suppose that $q_{\varepsilon}$ and $q_{\varepsilon}^{\prime}$ are positive quadratic forms for each $\varepsilon>0$ with domains dom $q_{\varepsilon}$ and $\operatorname{dom} q_{\varepsilon}^{\prime}$ such that the associated self-adjoint operators $Q_{\varepsilon}$ and $Q_{\varepsilon}^{\prime}$ have purely discrete spectrum denoted by $\lambda_{k}\left(Q_{\varepsilon}\right)$ and $\lambda_{k}\left(Q_{\varepsilon}^{\prime}\right)$ (as in the previous section).

1.4.1. Definition. A family $\left(u_{\varepsilon}\right)$ with $u_{\varepsilon} \in \operatorname{dom} q_{\varepsilon}$ will be called $\left(q_{\varepsilon}\right)$-bounded if there exists a constant $c>0$ such that

$$
\left\|u_{\varepsilon}\right\|_{q_{\varepsilon}}^{2}=\left\|u_{\varepsilon}\right\|_{\mathscr{C}_{\varepsilon}}^{2}+q_{\varepsilon}\left(u_{\varepsilon}\right) \leq c
$$

for all $\varepsilon>0$. 
The following theorem is a simple consequence of the Min-max Principle for discrete eigenvalues:

1.4.2. Theorem (Main Lemma). With the notation from above, for each $\varepsilon>0$ let

$$
\Phi_{\varepsilon}: \operatorname{dom} q_{\varepsilon} \longrightarrow \operatorname{dom} q_{\varepsilon}^{\prime}
$$

be a linear map which satisfies the following conditions for each $\left(q_{\varepsilon}\right)$-bounded family $\left(u_{\varepsilon}\right)$ :

1. $\lim _{\varepsilon \rightarrow 0}\left(\left\|\Phi_{\varepsilon} u_{\varepsilon}\right\|_{\mathscr{H}_{\varepsilon}^{\prime}}^{2}-\left\|u_{\varepsilon}\right\|_{\mathscr{H}_{\varepsilon}}^{2}\right)=0$ or $\left\|u_{\mathcal{\varepsilon}}\right\|_{\mathscr{H}_{\varepsilon}}^{2} \leq\left\|\Phi_{\varepsilon} u_{\varepsilon}\right\|_{\mathscr{H}_{\varepsilon}^{\prime}}^{2}$.

2. $\lim _{\varepsilon \rightarrow 0}\left(q_{\varepsilon}^{\prime}\left(\Phi_{\varepsilon} u_{\varepsilon}\right)-q_{\varepsilon}\left(u_{\varepsilon}\right)\right)=0$ or $q_{\varepsilon}\left(u_{\varepsilon}\right) \geq q_{\varepsilon}^{\prime}\left(\Phi_{\varepsilon} u_{\varepsilon}\right)$.

3. There exist constants $c_{k}>0$ such that $\lambda_{k}\left(q_{\varepsilon}\right) \leq c_{k}$ for each $\varepsilon>0$.

Then there exists for each $k \in \mathbb{N}$ a function $\delta_{k}(\varepsilon) \geq 0$ converging to 0 as $\varepsilon \rightarrow 0$ with

$$
\lambda_{k}\left(Q_{\varepsilon}^{\prime}\right) \leq \lambda_{k}\left(Q_{\varepsilon}\right)+\delta_{k}(\varepsilon)
$$

for small enough $\varepsilon>0$. If both inequalities in condition 1 and 2 are satisfied condition 3 is unnecessary and we can choose $\delta_{k}(\varepsilon)=0$.

Note that the convergence in Conditions 1 and 2 may depend on the family $\left(u_{\varepsilon}\right)$. In [Fuk87] a uniform control as in (1.15) is needed. Our result is in some sense more general, but the non-uniformness of the convergence complicates our proof (see the remark after the following proof).

Proof. Let $\left(\varphi_{k}^{\varepsilon}\right)_{k} \subset \operatorname{dom} q_{\varepsilon}$ be an orthonormal system of eigenvectors for the corresponding eigenvalues $\lambda_{k}\left(q_{\varepsilon}\right)$ and $u_{\varepsilon}=\sum_{j=1}^{k} \alpha_{j}^{\varepsilon} \varphi_{j}^{\varepsilon}$ be an element of the space $E_{k}^{\varepsilon}$ generated by the first $k$ eigenvalues. Condition 3 guarantees that $\left(\varphi_{j}^{\varepsilon}\right)_{\varepsilon}$ is a $q_{\varepsilon}$-bounded family, in particular, $q_{\varepsilon}\left(u_{\varepsilon}\right) \leq$ $c_{k}\left\|u_{\varepsilon}\right\|^{2}$. We have

$$
\begin{aligned}
\frac{q_{\varepsilon}^{\prime}\left(\Phi_{\varepsilon} u_{\varepsilon}\right)}{\left\|\Phi_{\varepsilon} u_{\varepsilon}\right\|^{2}}-\frac{q_{\varepsilon}\left(u_{\varepsilon}\right)}{\left\|u_{\varepsilon}\right\|^{2}} & = \\
& =\frac{1}{\left\|\Phi_{\varepsilon} u_{\varepsilon}\right\|^{2}}\left(\frac{q_{\varepsilon}\left(u_{\varepsilon}\right)}{\left\|u_{\varepsilon}\right\|^{2}}\left(\left\|u_{\varepsilon}\right\|^{2}-\left\|\Phi_{\varepsilon} u_{\varepsilon}\right\|^{2}\right)+\left(q_{\varepsilon}^{\prime}\left(\Phi_{\varepsilon} u_{\varepsilon}\right)-q_{\varepsilon}\left(u_{\varepsilon}\right)\right)\right) .
\end{aligned}
$$

Furthermore, we have the estimate

$$
\begin{aligned}
\left\|u_{\varepsilon}\right\|^{2}-\left\|\Phi_{\varepsilon} u_{\varepsilon}\right\|^{2}=\left|\sum_{i, j=1}^{k} \alpha_{i}^{\varepsilon} \overline{\alpha_{j}^{\varepsilon}}\left(\delta_{i j}-\left\langle\Phi_{\varepsilon} \varphi_{i}^{\varepsilon}, \Phi_{\varepsilon} \varphi_{j}^{\varepsilon}\right\rangle\right)\right| & \\
& \leq \delta_{k}^{\prime}(\varepsilon) \sum_{j=1}^{k}\left|\alpha_{j}^{\varepsilon}\right|^{2}=\delta_{k}^{\prime}(\varepsilon)\left\|u_{\varepsilon}\right\|^{2}
\end{aligned}
$$

with

$$
\delta_{k}^{\prime}(\varepsilon):=k \max _{i, j=1, \ldots, k}\left|\delta_{i j}-\left\langle\Phi_{\varepsilon} \varphi_{i}^{\varepsilon}, \Phi_{\varepsilon} \varphi_{j}^{\varepsilon}\right\rangle\right|
$$


Note that we have used the Cauchy-Schwarz Inequality in Estimate (1.9). We have $\delta_{k}^{\prime}(\varepsilon) \rightarrow 0$ by condition 1 and the Polarisation Identity (1.3):

$$
\begin{aligned}
\left|\delta_{i j}-\left\langle\Phi_{\varepsilon} \varphi_{i}^{\varepsilon}, \Phi_{\varepsilon} \varphi_{j}^{\varepsilon}\right\rangle\right|=\mid\left\langle\varphi_{i}^{\varepsilon}, \varphi_{j}^{\varepsilon}\right\rangle- & \left\langle\Phi_{\varepsilon} \varphi_{i}^{\varepsilon}, \Phi_{\varepsilon} \varphi_{j}^{\varepsilon}\right\rangle \mid \\
& \leq \frac{1}{4} \sum_{n=0}^{3}\left|\left\|\varphi_{i}^{\varepsilon}+\mathrm{i}^{n} \varphi_{j}^{\varepsilon}\right\|^{2}-\left\|\Phi_{\varepsilon} \varphi_{i}^{\varepsilon}+\mathrm{i}^{n} \Phi_{\varepsilon} \varphi_{j}^{\varepsilon}\right\|^{2}\right|
\end{aligned}
$$

Therefore the first term of the right hand side of Equation (1.8) will be smaller than $c_{k} \delta_{k}^{\prime}(\varepsilon)\left\|u_{\varepsilon}\right\|^{2} /\left\|\Phi_{\varepsilon} u_{\varepsilon}\right\|^{2}$. If we have only the second alternative of condition 1 we simply set $\delta_{k}^{\prime}(\varepsilon)=0$.

By a similar argument we can show the existence of a function $\delta_{k}^{\prime \prime}(\varepsilon) \geq 0$ converging to 0 as $\varepsilon \rightarrow 0$ with

$$
q_{\varepsilon}^{\prime}\left(\Phi_{\varepsilon} u_{\varepsilon}\right)-q_{\varepsilon}\left(u_{\varepsilon}\right) \leq \delta_{k}^{\prime \prime}(\varepsilon)\left\|u_{\varepsilon}\right\|^{2}
$$

From Estimate (1.9) we also conclude

$$
\left(1-\delta_{k}^{\prime}(\varepsilon)\right)\left\|u_{\varepsilon}\right\|^{2} \leq\left\|\Phi_{\varepsilon} u_{\varepsilon}\right\|^{2} .
$$

Assume that $\varepsilon$ is so small so that $\delta_{k}^{\prime}(\varepsilon)<1$ is valid. If we set

$$
\delta_{k}(\varepsilon):=\frac{1}{1-\delta_{k}^{\prime}(\varepsilon)}\left(c_{k} \delta_{k}^{\prime}(\varepsilon)+\delta_{k}^{\prime \prime}(\varepsilon)\right)
$$

then we can estimate (1.8) by

$$
\frac{q_{\varepsilon}^{\prime}\left(\Phi_{\varepsilon} u_{\varepsilon}\right)}{\left\|\Phi_{\varepsilon} u_{\varepsilon}\right\|^{2}}-\frac{q_{\varepsilon}\left(u_{\varepsilon}\right)}{\left\|u_{\varepsilon}\right\|^{2}} \leq \delta_{k}(\varepsilon)
$$

where we have used (1.9), (1.12) and (1.13).

Next we want to show that $\Phi_{\varepsilon}\left(E_{k}^{\varepsilon}\right)$ is $k$-dimensional. Suppose $u_{\varepsilon}=\sum_{j=1}^{k} \alpha_{j}^{\varepsilon} \varphi_{j}^{\varepsilon}$ with $\Phi_{\varepsilon} u_{\varepsilon}=0$. Then we have

$$
\sum_{j=1}^{k}\left|\alpha_{j}^{\varepsilon}\right|^{2}=\left\|u_{\varepsilon}\right\|^{2} \leq \frac{1}{1-\delta_{k}^{\prime}(\varepsilon)}\left\|\Phi_{\varepsilon} u_{\varepsilon}\right\|^{2}=0
$$

by Estimate (1.13) for small enough $\varepsilon$. So we have $\alpha_{j}^{\varepsilon}=0$ for all $j=1, \ldots, k$, thus $u_{\varepsilon}=0$ which implies the injectivity of $\Phi_{\varepsilon} \uparrow_{E_{k}^{\varepsilon}}$.

Finally, we apply the Min-max Principle (Theorem 1.3.3) to the quadratic form $q_{\varepsilon}^{\prime}$ and we obtain the estimate

$$
\lambda_{k}\left(Q_{\varepsilon}^{\prime}\right) \leq \lambda_{\Phi_{\varepsilon}\left(E_{k}^{\varepsilon}\right)}\left(q_{\varepsilon}^{\prime}\right)=\sup _{\substack{u_{\varepsilon} \in E_{k}^{\varepsilon} \\ u_{\varepsilon} \neq 0}} \frac{q_{\varepsilon}^{\prime}\left(\Phi_{\varepsilon} u_{\varepsilon}\right)}{\left\|\Phi_{\varepsilon} u_{\varepsilon}\right\|^{2}} \leq \sup _{\substack{u_{\varepsilon} \in E_{k}^{\varepsilon} \\ u_{\varepsilon} \neq 0}} \frac{q_{\varepsilon}\left(u_{\varepsilon}\right)}{\left\|u_{\varepsilon}\right\|^{2}}+\delta_{k}(\varepsilon)=\lambda_{k}\left(Q_{\varepsilon}\right)+\delta_{k}(\varepsilon)
$$

where we have used Estimate (1.14). Here, it is essential that $\delta_{k}(\varepsilon)$ is independent of $u_{\varepsilon}$. 
1.4.3. Remark. Note that we cannot apply directly our Conditions 1 and 2 to (1.8) since we need the error terms $\delta_{k}(\varepsilon)$ to be independent of $u_{\varepsilon}$. But in the conditions the convergence depends a priori on the family $\left(u_{\varepsilon}\right)$. This is the reason why we apply the conditions only to the eigenvectors $\left(\varphi_{i}^{\varepsilon}\right)$ but not directly to the family $\left(u_{\varepsilon}\right)$.

The alternative in the assumptions of the preceding theorem gives us the liberty either to prove the convergence or the inequality in Condition 1 resp. Condition 2 . We will use this fact to simplify our calculations when this theorem will be applied to parameter-dependent manifolds. We often use the preceding theorem with the following assumptions (for the definition of $\|\cdot\|_{q_{\varepsilon}}$ see (1.2)):

1.4.4. Corollary. Suppose there exists a positive function $\omega(\varepsilon) \rightarrow 0$ as $\varepsilon \rightarrow 0$ such that

$$
\begin{gathered}
\left|\left\|\Phi_{\varepsilon} u_{\varepsilon}\right\|_{\mathscr{H}_{\varepsilon}^{\prime}}^{2}-\left\|u_{\varepsilon}\right\|_{\mathscr{H}_{\varepsilon}}^{2}\right| \leq \omega(\varepsilon)\left\|u_{\varepsilon}\right\|_{q_{\varepsilon}}^{2} \\
q_{\varepsilon}\left(u_{\varepsilon}\right) \geq q_{\varepsilon}^{\prime}\left(\Phi_{\varepsilon} u_{\varepsilon}\right)
\end{gathered}
$$

for all $u_{\varepsilon} \in \mathscr{H}_{\varepsilon}$ and all $\varepsilon>0$ near 0. Suppose further that Condition 3 of Theorem 1.4.2 is fulfilled. Then the result of Theorem 1.4.2 holds.

The following simple result shows that the eigenvalues depend continuously on the norm and the quadratic form.

1.4.5. Lemma. Suppose that $\mathscr{H}$ and $\mathscr{H}^{\prime}$ are Hilbert spaces with the same underlying vector space structure. Suppose further that $q$ and $q^{\prime}$ are positive quadratic forms such that the corresponding operators $Q$ and $Q^{\prime}$ in $\mathscr{H}$ and $\mathscr{H}^{\prime}$ have purely discrete spectrum $\lambda_{k}(Q)$ and $\lambda_{k}\left(Q^{\prime}\right)$. If there exists a number $0<\eta \leq \frac{1}{2}$ such that

$$
\begin{aligned}
\left|\|u\|_{\mathscr{H}}^{2}-\|u\|_{\mathscr{H}^{\prime}}^{2}\right| & \leq \eta\|u\|_{\mathscr{H}}^{2} \\
\left|q(u)-q^{\prime}(u)\right| & \leq \eta q(u)
\end{aligned}
$$

for all $u \in \mathscr{H}$ then $\left|\lambda_{k}\left(Q^{\prime}\right)-\lambda_{k}(Q)\right| \leq 4 \eta$.

Proof. From the assumptions we obtain

$$
\frac{1-\eta}{1+\eta} \frac{q^{\prime}(u)}{\|u\|_{\mathscr{H}^{\prime}}^{2}} \leq \frac{q(u)}{\|u\|_{\mathscr{H}}^{2}} \leq \frac{1+\eta}{1-\eta} \frac{q^{\prime}(u)}{\|u\|_{\mathscr{H}^{\prime}}^{2}}
$$

The result follows by the Min-max Principle.

Next we consider how eigenvectors can be approximated in the context of parameterdependent Hilbert spaces. Suppose that $\mathscr{H}, \mathscr{H}_{n}$ are Hilbert spaces such that $\mathscr{H}_{n} \subset \mathscr{H}$ as vector spaces for all $n \in \mathbb{N}$, i.e., the inner product of $\mathscr{H}_{n}$ may depend on $n$. Suppose further that $q$ and $q_{n}$ are positive, closed quadratic forms in $\mathscr{H}$ and $\mathscr{H}_{n}$ such that $\operatorname{dom} q_{n} \subset \operatorname{dom} q$ for all $n \in \mathbb{N}$. Denote the corresponding operators by $Q$ and $Q_{n}$.

Let $\mathscr{D}$ be a form core of $q$ such that for every $v \in \mathscr{D}$ there exists a number $n_{0}=n_{0}(v) \in \mathbb{N}$ with $v \in \operatorname{dom} q_{n}$ for all $n \geq n_{0}$. 
1.4.6. Theorem. Suppose that there exist a constant $c>1$ and a sequence $\delta_{n} \rightarrow 0$ such that

$$
\begin{gathered}
\left\|u_{n}\right\|_{q}^{2} \leq c\left\|u_{n}\right\|_{q_{n}}^{2} \\
\left|\left\langle u_{n}, v\right\rangle_{\mathscr{H}_{n}}-\left\langle u_{n}, v\right\rangle_{\mathscr{H}}\right| \leq \delta_{n}\left(\left\|u_{n}\right\|_{q_{n}}^{2}+\|v\|_{q}^{2}\right) \\
\left|q_{n}\left(u_{n}, v\right)-q\left(u_{n}, v\right)\right| \leq \delta_{n}\left(\left\|u_{n}\right\|_{q_{n}}^{2}+\|v\|_{q}^{2}\right)
\end{gathered}
$$

for all $v \in \mathscr{D}, n \geq n_{0}(v)$ and $u_{n} \in \operatorname{dom} q_{n}$. Suppose further that $\lambda_{n} \rightarrow \lambda$ and that there exist $\varphi_{n} \in \operatorname{dom} Q_{n}$ such that

$$
Q_{n} \varphi_{n}=\lambda_{n} \varphi_{n}, \quad\left\|\varphi_{n}\right\|_{\mathscr{H}_{n}}=1
$$

Then there exists a subsequence $\left(\varphi_{n_{m}}\right)_{m}$ of $\left(\varphi_{n}\right)_{n}$ and an element $\varphi \in \operatorname{dom} Q$ such that $\varphi_{n_{m}} \rightarrow \varphi$ weakly in $\operatorname{dom} q$, weakly in $\mathscr{H}$ and

$$
Q \varphi=\lambda \varphi
$$

Note that we have not shown that the limit satisfies $\varphi \neq 0$. To prove this will be the main difficulty in Chapter 8. Most of Section 2.4 is dedicated to deliver tools to solve this problem.

Proof. By Assumptions (1.19) and (1.22) we estimate

$$
\left\|\varphi_{n}\right\|_{\mathscr{H}}^{2} \leq\left\|\varphi_{n}\right\|_{q}^{2} \leq c\left\|\varphi_{n}\right\|_{q_{n}}^{2}=c\left(1+\lambda_{n}\right)\left\|\varphi_{n}\right\|_{\mathscr{H}_{n}}^{2}=c\left(1+\lambda_{n}\right) .
$$

Therefore, $\left(\varphi_{n}\right)_{n}$ is a norm-bounded sequence in $\mathscr{H}$. By Theorem 1.1.1 there exist a subsequence $\left(\varphi_{n_{m}}\right)_{m}$ and an element $\varphi \in \mathscr{H}$ such that $\varphi_{n_{m}} \rightarrow \varphi$ weakly in dom $q$. Since the embedding map $\operatorname{dom} q \hookrightarrow \mathscr{H}$ is norm-continuous it is also continuous with respect to the weak topologies on both spaces (see Lemma 1.2.1). We therefore conclude

$$
\left\langle\varphi_{n_{m}}, v\right\rangle_{\mathscr{H}} \rightarrow\langle\varphi, v\rangle_{\mathscr{H}} \quad \text { and } \quad q\left(\varphi_{n_{m}}, v\right) \rightarrow q(\varphi, v)
$$

for all $v \in \operatorname{dom} q$. Furthermore,

$$
\begin{aligned}
\left|q(\varphi, v)-\lambda\langle\varphi, v\rangle_{\mathscr{H}}\right| \leq\left|q\left(\varphi-\varphi_{n_{m}}, v\right)\right|+\left|q\left(\varphi_{n_{m}}, v\right)-q_{n_{m}}\left(\varphi_{n_{m}}, v\right)\right|+ \\
\quad+\lambda_{n_{m}}\left|\left\langle\varphi_{n_{m}}, v\right\rangle_{\mathscr{H}_{n}}-\left\langle\varphi_{n_{m}}, v\right\rangle_{\mathscr{H}}\right|+\lambda_{n_{m}}\left|\left\langle\varphi_{n_{m}}-\varphi, v\right\rangle_{\mathscr{H}}\right|+\left|\lambda_{n_{m}}-\lambda\right|\left|\langle\varphi, v\rangle_{\mathscr{H}}\right|
\end{aligned}
$$

for all $v \in \mathscr{D}$ and $m \in \mathbb{N}$ such that $n_{m} \geq n_{0}(v)$. Clearly, the last term of the right hand side converges to 0 as $m \rightarrow \infty$. By (1.24) the same is true for the first and forth term. The second and third term can be estimated by

$$
\left(1+\lambda_{n_{m}}\right) \delta_{n_{m}}\left(1+\lambda_{n_{m}}\|v\|_{q}^{2}\right) \rightarrow 0
$$

with regard to assumptions (1.20), (1.21) and (1.22). Therefore we have shown that $\varphi \in \operatorname{dom} Q$ and (1.23).

We give another criterion for the assumptions made in the preceding theorem: 
1.4.7. Lemma. Suppose there exists a sequence $\eta_{n} \rightarrow 0$ such that

$$
\begin{gathered}
\left|\left\|u_{n}\right\|_{\mathscr{H}^{2}}^{2}-\left\|u_{n}\right\|_{\mathscr{H}_{n}}^{2}\right| \leq \eta_{n}\left\|u_{n}\right\|_{q_{n}}^{2} \\
\left|q\left(u_{n}\right)-q_{n}\left(u_{n}\right)\right| \leq \eta_{n}\left\|u_{n}\right\|_{q_{n}}^{2}
\end{gathered}
$$

for all $n \in \mathbb{N}$ and $u_{n} \in \operatorname{dom} q_{n}$. Then conditions (1.19), (1.20) and (1.21) are fulfilled with $\delta_{n}:=\frac{2 \eta_{n}}{1-2 \eta_{n}}$. Furthermore there exists $c>1$ such that

$$
\left\|u_{n}\right\|_{q_{n}}^{2} \leq c\left\|u_{n}\right\|_{q}^{2}
$$

for all $u_{n} \in \operatorname{dom} q_{n}$.

Proof. From (1.25) and (1.26) one gets

$$
\left\|u_{n}\right\|_{q}^{2} \leq\left(1+2 \eta_{n}\right)\left\|u_{n}\right\|_{q_{n}}^{2} \quad \text { and } \quad\left\|u_{n}\right\|_{q_{n}}^{2} \leq \frac{1}{1-2 \eta_{n}}\left\|u_{n}\right\|_{q}^{2}
$$

Therefore (1.19) and (1.27) follow. Furthermore

$$
\begin{aligned}
& \mid\left\langle u_{n}, v\right\rangle_{\mathscr{H}_{n}}-\left\langle u_{n}, v\right\rangle_{\mathscr{H}} \mid \leq \frac{1}{4} \sum_{k=0}^{3}\left(\left\|u_{n}+\mathrm{i}^{k} v\right\|_{\mathscr{H}_{n}}^{2}-\left\|u_{n}+\mathrm{i}^{k} v\right\|_{\mathscr{H}^{\prime}}^{2}\right) \leq \\
& \quad \leq \frac{\eta_{n}}{4} \sum_{k=0}^{3}\left\|u_{n}+\mathrm{i}^{k} v\right\|_{\mathscr{H}_{n}}^{2} \leq 2 \eta_{n}\left(\left\|u_{n}\right\|_{\mathscr{H}_{n}}^{2}+\|v\|_{q_{n}}^{2}\right) \leq \frac{2 \eta_{n}}{1-2 \eta_{n}}\left(\left\|u_{n}\right\|_{\mathscr{H}_{n}}^{2}+\|v\|_{q}^{2}\right) .
\end{aligned}
$$

by using the Polarisation Identity (1.3), Estimate (1.27) and Assumption (1.25). We therefore have proven (1.20). Condition (1.21) can be shown in a similar way.

\subsection{Interchange of norm and quadratic form}

The next result will be needed in Section 7.2. Suppose we are given a strictly positive closed quadratic form $q$ in the Hilbert space $\mathscr{H}$, i.e., 0 is not in the spectrum of the corresponding operator $Q$. Sometimes it is useful to interchange the rôle of the (squared) norm and the quadratic form $q$, i.e., we define a new Hilbert space $\widetilde{\mathscr{H}}$ on dom $q$ with norm $q$ and regard the original norm $\|\cdot\|_{\mathscr{H}}^{2}$ as a quadratic form with corresponding operator $\widetilde{Q}$. Note that the new norm $q$ on $\operatorname{dom} q$ is different from the norm $\|u\|_{q}^{2}=\|u\|_{\mathscr{H}}^{2}+q(u)$ arising from the inner product in (1.2).

We have the following correspondence between the spectrum of $Q$ and the spectrum of $\widetilde{Q}$ :

1.5.1. Lemma. Let $Q$ be a positive operator with $0 \notin \operatorname{spec} Q$ in a Hilbert space $\mathscr{H}$ with corresponding quadratic form $q$ on $\widetilde{\mathscr{H}}:=\operatorname{dom} q$. We further suppose that $Q$ has purely discrete spectrum, i.e., $\operatorname{spec} Q=\left\{\lambda_{k} \mid k \in \mathbb{N}\right\}$ with $0<\lambda_{k} \nearrow \infty$ as $k \rightarrow \infty$.

Then $\widetilde{\mathscr{H}}$ equipped with $\|u\|_{\widetilde{\mathscr{H}}}^{2}:=q(u)$ is a Hilbert space and the operator $\widetilde{Q}$ corresponding to the quadratic form $\widetilde{q}(u):=\|u\|_{\mathscr{H}}^{2}$ for $u \in \widetilde{\mathscr{H}}$ in the Hilbert space $\widetilde{\mathscr{H}}$ has spectrum

$$
\operatorname{spec} \widetilde{Q}=\left\{\frac{1}{\lambda_{k}} \mid k \in \mathbb{N}\right\} \cup\{0\} \text {. }
$$

In particular, $\widetilde{Q}$ is compact. 
Proof. Clearly, $\langle u, v\rangle_{\widetilde{\mathscr{C}}}=q(u, v)$ is sesquilinear and positive definite. The Min-max Principle implies

$$
\lambda_{1}\|u\|_{\mathscr{H}}^{2} \leq q(u)
$$

and

$$
\frac{\lambda_{1}}{1+\lambda_{1}}\left(q(u)+\|u\|_{\mathscr{H}}^{2}\right) \leq q(u) \leq q(u)+\|u\|_{\mathscr{H}}^{2} .
$$

Since $\lambda_{1}>0$ and $q$ is a closed form, $\|\cdot\|_{\widetilde{\mathscr{H}}}^{2}$ is equivalent to a complete norm.

Let $\left(u_{k}\right)_{k}$ be an orthonormal basis of $\mathscr{H}$ consisting of eigenvectors of $Q$ with corresponding eigenvalues $\lambda_{k}$. Then $\widetilde{u}_{k}:=\frac{1}{\sqrt{\lambda_{k}}} u_{k}$ is orthonormal in $\widetilde{\mathscr{H}}$. Suppose $\widetilde{v}$ is orthogonal in $\widetilde{\mathscr{H}}$ to $\widetilde{u}_{k}$ for all $k \in \mathbb{N}$. Then we have

$$
0=\left\langle\widetilde{u}_{k}, \widetilde{v}\right\rangle_{\mathscr{\mathscr { H }}}=q\left(\widetilde{u}_{k}, \widetilde{v}\right)=\sqrt{\lambda_{k}}\left\langle u_{k}, \widetilde{v}\right\rangle_{\mathscr{H}}
$$

for every $k$. Since $\lambda_{k}>0$ it follows that $\widetilde{v}$ is orthogonal to every $u_{k}$ in $\mathscr{H}$, thus $\widetilde{v}=0$. We have therefore shown that $\left(\widetilde{u}_{k}\right)_{k}$ is a orthonormal basis of $\widetilde{\mathscr{H}}$.

We further have

$$
\left\langle\widetilde{Q} \widetilde{u}_{k}, \widetilde{v}\right\rangle_{\widetilde{\mathscr{H}}}=\widetilde{q}\left(\widetilde{u}_{k}, \widetilde{v}\right)=\left\langle\widetilde{u}_{k}, \widetilde{v}\right\rangle_{\mathscr{H}}=\frac{1}{\lambda_{k}}\left\langle Q \widetilde{u}_{k}, \widetilde{v}\right\rangle_{\mathscr{H}}=\frac{1}{\lambda_{k}} q\left(\widetilde{u}_{k}, \widetilde{v}\right)=\frac{1}{\lambda_{k}}\left\langle\widetilde{u}_{k}, \widetilde{v}\right\rangle_{\widetilde{\mathscr{H}}}
$$

for every $\widetilde{v} \in \widetilde{\mathscr{H}}$. We have shown that $\widetilde{u}_{k}$ is an eigenvector with eigenvalue $\frac{1}{\lambda_{k}}$ for $\widetilde{Q}$ in $\widetilde{\mathscr{H}}$. 


\section{Analysis on manifolds}

In this chapter we quote the necessary results needed later on. Sometimes, proofs are given if we need special features which are not in standard textbooks. In the sequel we assume that our manifolds are oriented and connected unless stated otherwise.

\subsection{Spaces of square integrable functions}

Suppose $M$ is a Riemannian manifold with metric $g$. Let $\varphi: U \longrightarrow V$ be a chart where $U \subset M$ and $V \subset \mathbb{R}^{d}$ are open subsets. Denote by $\mathrm{d} x$ the unique volume measure given locally by

$$
\int_{M} u:=\int_{M} u(x) \mathrm{d} x=\int_{\varphi U} u(y)(\operatorname{det} g(y))^{\frac{1}{2}} \mathrm{~d} y
$$

for all integrable functions $u: U \longrightarrow \mathbb{R}$. Here, $\operatorname{det} g(y)$ denotes the determinant of the usual Riemannian metric coefficients $\left(g_{i j}(y)\right)_{i j}$ of $g$ in the chart $U$. Furthermore dy denotes the Lebesgue measure on $\mathbb{R}^{d}$. Note that the volume measure $\mathrm{d} x$ exists even on non-orientable manifolds, see [BGM71], but here we will restrict ourselves to oriented manifolds. Note that we do not distinguish between $u$ and its representation in a chart $u \circ \varphi^{-1}$, i.e., we identify $x \in U$ with $y=\varphi(x) \in V$ and write $u(y)$ instead of $u\left(\varphi^{-1} y\right)$.

If $M$ has a boundary $\partial M \neq \emptyset$ we always assume that $\partial M$ is (piecewise) smooth and endowed with its natural Riemannian metric and volume measure inherited from $M$. Denote by $\stackrel{\circ}{M}$ the open set $M \backslash \partial M$. If $\partial M$ is smooth we need charts $\varphi: U \longrightarrow V$ with open subsets $V$ of the half space $\mathbb{R}_{+}^{d}:=\left\{x \in \mathbb{R}^{d} \mid x_{d} \geq 0\right\}$.

Let $p_{E}: E \longrightarrow M$ be a hermitian vector bundle, i.e., a vector bundle with inner product $\langle\cdot, \cdot\rangle_{E_{x}}$ in each fiber $E_{x}$ of $E$. In particular, $E_{x}$ is a complex Hilbert space of constant finite dimension $r$. We often write $\langle\cdot, \cdot\rangle$ for the inner product and $|\cdot|$ for the corresponding norm (suppressing $E_{x}$ in the notation).

In our applications, $E$ will be the exterior tangent bundle $\mathbf{A}^{p} T M$ of degree $p=0, \ldots, d$ (with the convention $\mathbf{A}^{0} T M:=M \times \mathbb{C}$ and $\left.T^{*} M:=\mathbf{A}^{1} T M\right)$. This bundle inherits in a natural way a hermitian structure $\langle\cdot, \cdot\rangle_{\mathbf{A}^{p} T M}=g_{x}^{\mathbf{A}^{p} T M}(\cdot, \cdot)$ on each fibre $\mathbf{A}^{p} T M_{x}=\mathbf{A}^{p} T_{x} M$ from the tangent bundle $T M$ with inner product $\langle\cdot, \cdot\rangle_{T_{x} M}=g_{x}(\cdot, \cdot)$ on the (complexified) fibre $T_{x} M$. If $\left(g^{i j}\right)_{i j}$ denotes the inverse matrix of $\left(g_{i j}\right)_{i j}$ in a given chart $y=\varphi(x)$, we have the local expression

$$
\begin{aligned}
\left(g^{\mathbf{A}^{p} T M}\right)_{y}^{i_{1}, \ldots, i_{p}, j_{1}, \ldots, j_{p}}=\frac{1}{p !} g_{y}^{\mathbf{A}^{p} T M}\left(\mathrm{~d} y^{i_{1}} \wedge \cdots \wedge \mathrm{d} y^{i_{p}}, \mathrm{~d} y^{j_{1}}\right. & \left.\wedge \cdots \wedge \mathrm{d} y^{j_{p}}\right)= \\
& =\sum_{\sigma \in \mathscr{S} p} \operatorname{sgn} \sigma g_{y}^{i_{1} j_{\sigma(1)}} \ldots g_{y}^{i_{p} j_{\sigma(p)}}
\end{aligned}
$$


where $\mathscr{S}^{p}$ denotes the group of all permutations of the set $\{1, \ldots, p\}$. Note that the factor $\frac{1}{p !}$ is necessary to have a definition consistent with (2.20).

A section in $E$ is a differential map $u: M \longrightarrow E$ with $p_{E}(u(x))=x$, i.e., a smooth map which associates to each point $x$ an element $u(x) \in E_{x}$. If $\psi: U \longrightarrow V \times \mathbb{C}^{r}$ denotes a trivalising map of $E$, we can identify the restricted section $\left.u\right|_{U}$ with a map $V \longrightarrow \mathbb{C}^{r}$, the local representation of the section in the chart $\varphi: U \longrightarrow V$. Let $C_{c}^{k}(E)$ denote the space of all compactly supported $C^{k}$-sections of $E$ such that the support does not intersect the boundary of $M$. For $u, v \in C_{c}^{\infty}(E)$, we define the inner product

$$
\langle u, v\rangle_{L_{2}(E)}:=\int_{M}\langle u(x), v(x)\rangle_{E_{x}} \mathrm{~d} x
$$

We denote by $L_{2}(E)$ the completion of $C_{c}^{\infty}(E)$ under the corresponding norm. We therefore obtain a Hilbert space. As an example, $L_{2}\left(\mathbf{A}^{p} T M\right)$ is the space of all square integrable differential forms of degree $p$. In the case of the trivial bundle $\mathbf{A}^{0} T M=M \times \mathbb{C}$ we write $L_{2}(M)$ instead of $L_{2}(M \times \mathbb{C})$ for the space of all square integrable functions on $M$ and similarly for other function spaces. Therefore we obtain

$$
\langle u, v\rangle_{L_{2}(M)}=\int_{M} u(x) \overline{v(x)} \mathrm{d} x
$$

We say that $u$ lies in $L_{2, \text { loc }}(E)$ if $u \uparrow_{K}$ lies in $L_{2}\left(E \uparrow_{K}\right)$ for all compact subsets $K$ of $M$. Here, $E \uparrow_{K}$ denotes the bundle $E$ restricted to $K$. We say that $u_{n} \in L_{2, \text { loc }}(E)$ converges strongly in $L_{2, \mathrm{loc}}(E)$ if $u_{n} \uparrow_{K}$ converges strongly in $L_{2}\left(E \uparrow_{K}\right)$ for all compact subsets $K$ of $M$.

\subsection{Sobolev spaces}

For more details see e.g., [Hör85, Appendix B], [Gi195], [Ros97] or [Heb96].

Define the $k$-th Sobolev space on the open subset $V$ of $\mathbb{R}^{d}$ as the completion of the space of smooth maps $u: V \longrightarrow \mathbb{C}^{r}$ such that

$$
\|u\|_{\mathscr{H}^{k}(V)}^{2}:=\sum_{|\kappa| \leq k}\left\|\partial_{\kappa} u\right\|_{L_{2}(V)}^{2}
$$

is finite under the norm defined by (2.4). Denote this space by $\mathscr{H}^{k}(V)$. Here, $\|u\|_{L_{2}(V)}^{2}=$ $\sum_{i}\left\|u_{i}\right\|_{L_{2}(V)}^{2}$ denotes the (square of the) usual $L_{2}$-norm of the vector-valued function $u$, and $\partial_{\kappa} u$ the derivative in each component of $u$ with respect to the multi-index $\kappa$. Denote by $\mathscr{H}^{k}(V)$ the closure of $C_{c}^{\infty}(V)$ in $\mathscr{H}^{k}(V)$.

To define the $k$-th Sobolev space on vector bundles $E \longrightarrow M$ we have to use a localisation procedure. Let $\left(\chi_{\alpha}\right)_{\alpha \in A}$ be a partition of unity subordinate to the atlas $\mathscr{A}=\left(\varphi_{\alpha}: U_{\alpha} \longrightarrow\right.$ $\left.V_{\alpha}\right)_{\alpha \in A}$ of $M$ where $\varphi_{\alpha}$ is a chart and $\left(U_{\alpha}\right)_{\alpha}$ a locally finite cover. Denote by $C^{k}(E)$ the space of all functions of class $C^{k}$ up to the boundary. Then $\mathscr{H}^{k}(E)$ is the completion of the space of all $u \in C^{\infty}(E)$ such that

$$
\|u\|_{\mathscr{H}^{k}(E)}^{2}:=\|u\|_{\mathscr{H}^{k}(E), \mathscr{A}}^{2}:=\sum_{\alpha}\left\|\chi_{\alpha} u_{\alpha}\right\|_{\mathscr{H}^{k}\left(V_{\alpha}\right)}^{2}
$$




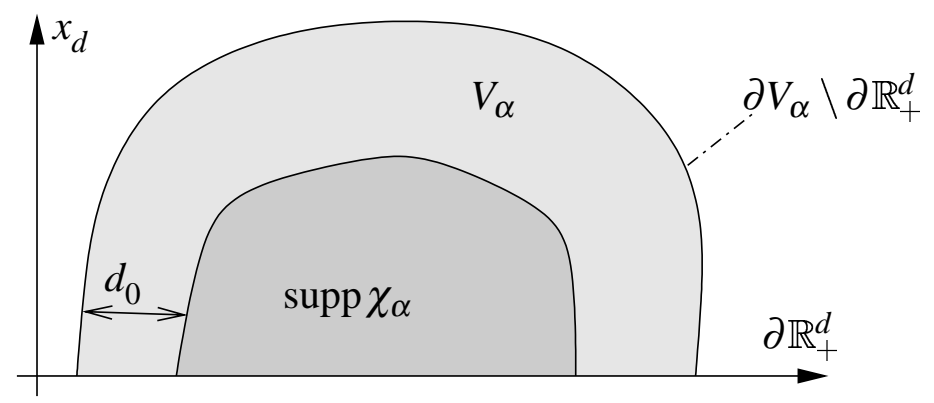

Figure 2.1: The function $\chi_{\alpha}$ with distance $d_{0}$ from the boundary.

is finite. Here, $u_{\alpha}: V_{\alpha} \longrightarrow \mathbb{C}^{r}$ denotes the local representation of the section $u$ in the chart $\varphi_{\alpha}$. Again, $\mathscr{H}^{k}(E)$ is the closure of $C_{c}^{\infty}(E)$ in $\mathscr{H}^{k}(E)$. Note that this norm depends on the choice of the cover and the atlas. Nevertheless, we give a criterion under which the norms are equivalent (see Lemma 2.2.3). Define the supremum norm on the space $C^{k}(V)$ of all $C^{k}$ functions $u: V \longrightarrow \mathbb{C}^{d}$ by

$$
\|u\|_{C(V)}:=\sup _{x \in V} \max _{i}\left|u_{i}(x)\right| \quad \text { and } \quad\|u\|_{C^{k}(V)}:=\max _{|\kappa| \leq k}\left\|\partial_{\kappa} u\right\|_{C(V)} .
$$

2.2.1. Definition. Let $k>0$. An atlas $\mathscr{A}=\left(\varphi_{\alpha}: U_{\alpha} \longrightarrow V_{\alpha}\right)_{\alpha \in A}$ will be called $C^{k}$-bounded (with bound $c_{0}$ and maximal number of neighbours $N$ ) if $V_{\alpha}$ is relatively compact for all $\alpha \in A$ and if there exists a constant $c_{0}$ and a number $N \in \mathbb{N}$ such that

$$
\begin{gathered}
\sup _{\alpha, \alpha^{\prime} \in A}\left\|\varphi_{\alpha} \circ \varphi_{\alpha^{\prime}}^{-1}\right\|_{C^{k}\left(V_{\alpha} \cap V_{\alpha^{\prime}}\right)}=c_{0}<\infty \\
\operatorname{card}\left\{\alpha^{\prime} \in A \mid U_{\alpha} \cap U_{\alpha^{\prime}} \neq \emptyset\right\} \leq N \quad \text { for all } \alpha \in A .
\end{gathered}
$$

A partition of unity $\left(\chi_{\alpha}\right)$ subordinate to the $C^{k}$-bounded atlas $\mathscr{A}$ will be called $C^{k}$-bounded with respect to the atlas $\mathscr{A}$ (with bound $c_{0}$ and distance $d_{0}$ from the boundary) if there exist constants $c_{0}, d_{0}>0$ such that

$$
\begin{gathered}
\sup _{\alpha \in A}\left\|\chi_{\alpha}\right\|_{C^{k}\left(V_{\alpha}\right)}=c_{0}<\infty \\
\inf _{\alpha \in A} \operatorname{dist}\left(\operatorname{supp} \chi_{\alpha}, \partial V_{\alpha} \backslash \partial \mathbb{R}_{+}^{d}\right)=d_{0}>0 .
\end{gathered}
$$

Note that on a compact manifold, every (finite) partition of unity is $C^{k}$-bounded. In Section 3.6 we show that $C^{k}$-bounded partitions of unity also exist on periodic (non-compact) manifolds.

Condition (2.10) says that the distance of $\operatorname{supp} \chi_{\alpha}$ from the "artificial" boundary $\partial V_{\alpha} \backslash \partial \mathbb{R}_{+}^{d}$ of the chart has a lower bound (see Figure 2.1). This will be needed to apply regularity theory.

Condition (2.8) says, roughly speaking, that every set $U_{\alpha}$ has at most $N$ neighbours. This is necessary in the following lemma:

2.2.2. Lemma. Let $\left(\chi_{\alpha}\right)$ be a partition of unity subordinate to the cover $\left(U_{\alpha}\right)$ of $M$ with at most $N$ neighbours, i.e., (2.8) is satisfied. Suppose further that $D$ is an operator in $L_{2}(M)$. 
Then we have

$$
\begin{gathered}
\|D u\|_{L_{2}(M)}^{2} \leq 2 N \sum_{\alpha}\left\|D \chi_{\alpha} u\right\|_{L_{2}(M)}^{2} \\
\frac{1}{2 N}\|u\|_{L_{2}(M)}^{2} \leq \sum_{\alpha}\left\|\chi_{\alpha} u\right\|_{L_{2}\left(U_{\alpha}\right)}^{2} \leq\|u\|_{L_{2}(M)}^{2}
\end{gathered}
$$

for all $u \in \operatorname{dom} D$ with $\chi_{\alpha} u \in \operatorname{dom} D$ resp. $u \in L_{2}(M)$.

Proof. We estimate

$$
\begin{aligned}
\|D u\|^{2}= & \sum_{\substack{\alpha, \alpha^{\prime} \\
U_{\alpha} \cap U_{\alpha^{\prime}} \neq \emptyset}}\left\langle D\left(\chi_{\alpha} u\right), D\left(\chi_{\alpha^{\prime}} u\right)\right\rangle \leq \\
& \leq \sum_{\substack{\alpha, \alpha^{\prime} \\
U_{\alpha} \cap U_{\alpha^{\prime}} \neq \emptyset}}\left(\left\|D\left(\chi_{\alpha} u\right)\right\|^{2}+\left\|D\left(\chi_{\alpha^{\prime}} u\right)\right\|^{2}\right) \leq 2 N \sum_{\alpha}\left\|D\left(\chi_{\alpha} u\right)\right\|^{2}
\end{aligned}
$$

by using the Cauchy-Young Inequality (1.1). The second statement follows form the first with $D=$ id and by the fact that

$$
\sum_{\alpha}\left\|\chi_{\alpha} u\right\|_{L_{2}\left(U_{\alpha}\right)}^{2} \leq \sum_{\alpha} \int_{U_{\alpha}} \chi_{\alpha}|u|^{2}=\|u\|_{L_{2}(M)}^{2}
$$

since $\chi_{\alpha}^{2} \leq \chi_{\alpha} \leq 1$.

Now we give a criterion under which the different Sobolev norms are equivalent.

2.2.3. Lemma. Suppose that $\left(\chi_{\alpha}\right)$ and $\left(\tilde{\chi}_{\widetilde{\alpha}}\right)$ are $C^{k}$-bounded partitions of unity subordinate to the $C^{k}$-bounded atlases $\mathscr{A}$ and $\widetilde{\mathscr{A}}$ with at most $N$ neighbours and $C^{k}$-bound $c_{0}$. Then there exists a constant $c=c\left(N, c_{0}\right) \geq 1$ such that

$$
\frac{1}{c}\|u\|_{\mathscr{H}^{k}(E), \mathscr{A}} \leq\|u\|_{\mathscr{H}^{k}(E), \widetilde{A}} \leq c\|u\|_{\mathscr{H}^{k}(E), \mathscr{A}} \cdot
$$

for all $u \in \mathscr{H}^{k}(E)$. In particular, the estimate holds on a compact manifold.

Proof. Note that Theorem B.1.8 of [Hör85] is needed.

The following theorem follows easily from the Euclidian case:

2.2.4. Theorem (Rellich-Kondrachov Compactness Theorem). Suppose that E is a Hermitian vector bundle over the compact manifold $M$. Suppose further that $k_{1}>k_{2}$. Then the embedding

$$
\imath: \mathscr{H}^{k_{1}}(E) \hookrightarrow \mathscr{H}^{k_{2}}(E)
$$

is compact, i.e., every sequence $\left(u_{n}\right)_{n}$ converging weakly in the Hilbert space $\mathscr{H}^{k_{1}}(E)$ to an element $u \in \mathscr{H}^{k_{1}}(E)$ converges in norm in $\mathscr{H}^{k_{2}}(E)$.

Finally, we quote the following theorem:

2.2.5. Theorem. Let $M$ be a Riemannian manifold with smooth boundary $\partial M$ and let $E$ be a Hermitian bundle over $M$. Then the restriction $u \uparrow_{\partial M}$ of a section $u \in \mathscr{H}^{1}(E)$ is well-defined and lies in $L_{2}\left(E \uparrow_{\partial M}\right)$.

Proof. (see [Ste70] or [Hör85, Appendix B]) 


\subsection{The Laplacian on a manifold}

Now we can define the Laplacian on a manifold. Many of the following statements on the Laplacian can be found in [Cha84], [Tay96] or in [DS99, pp. 30-75], for example.

Let $M$ be a (possibly non-compact) Riemannian manifold without boundary. We denote the exterior derivative on $M$ by

$$
\mathrm{d}: C_{c}^{\infty}\left(\mathbf{A}^{p} T M\right) \longrightarrow C_{c}^{\infty}\left(\mathbf{A}^{p+1} T M\right)
$$

and by $\mathrm{d}^{*}$ its formal adjoint with respect to the inner product given by (2.2). Define a quadratic form in the Hilbert space $L_{2}\left(\mathbf{A}^{p} T M\right)$ by

$$
q_{\mathbf{A}^{p} T M}(u):=\int_{M}\left(|\mathrm{~d} u|^{2}+\left|\mathrm{d}^{*} u\right|^{2}\right)
$$

for $u \in C_{c}^{\infty}\left(\mathbf{A}^{p} T M\right)$. Since $q_{\mathbf{A}^{p} T M}$ is a densely defined, positive form the closure exists and will also be denoted by $q_{\mathbf{A}^{p} T M}$. Cleary, $C_{c}^{\infty}\left(\mathbf{A}^{p} T M\right)$ is a form core. We sometimes call the integral in (2.13) the energy integral of $\mathbf{A}^{p} T M$ and its value the energy of $u$.

If $M$ is complete, i.e., $M$ is complete as a metric space or, equivalently, every maximal geodesic is defined on $\mathbb{R}$, one can prove that $\mathscr{H}^{1}(M)=\operatorname{dom} q_{M}$ (this is a part of Gaffney's Theorem, see [DS99, p. 53]). If the metric is uniformly elliptic with respect to an atlas $\mathscr{A}$ then the norms $\|\cdot\|_{\mathscr{H}^{1}(M), \mathscr{A}}$ and $\|\cdot\|_{q_{M}}$ are equivalent. In particular, this is the case if $M$ is compact.

If we deal with functions, i.e., $p=0$, the quadratic form is given by

$$
q_{M}(u)=\int_{M}|\mathrm{~d} u|^{2}=\int_{U} \sum_{i, j=1}^{d} g_{y}^{i j} \partial_{i} u(y) \partial_{j} \overline{u(y)}(\operatorname{det} g(y))^{\frac{1}{2}} \mathrm{~d} y .
$$

Here, the second equality is valid if $\operatorname{supp} u$ lies in the coordinate chart $U$ (see (2.1)). Note that $\mathrm{d} u=\sum_{i} \partial_{i} u \mathrm{~d} y^{i}$.

2.3.1. Definition. Suppose $M$ is a (possibly non-compact) manifold without boundary. Let $\Delta_{\mathbf{A}^{p} T M}$ be the operator corresponding to the closed, positive sesquilinear form $q_{\mathbf{A}^{p} T M}$ (see Theorem 1.2.2), called the Laplacian on $\mathbf{A}^{p} T M$. For the Laplacian on functions we simply write $\Delta_{M}$. If the context is clear, we sometimes write $\Delta$.

Formally, we have $\Delta_{\mathbf{A}^{p} T M}=\mathrm{d}^{*} \mathrm{~d}+\mathrm{dd}^{*}$ and $\Delta_{M}=\mathrm{d}^{*} \mathrm{~d}$.

Note that if $M$ is complete, the Laplacian on functions is unique in the following sense: By Gaffney's Theorem, $\left.\Delta_{M}\right\rceil_{C_{c}^{\infty}(M)}$ is essentially self-adjoint, i.e., the smallest closed extension is self-adjoint and agrees with our definition of the Laplacian.

If we deal with functions $(p=0)$, we specify different boundary conditions. Therefore we suppose that $M$ is a compact manifold with boundary $\partial M \neq \emptyset$. We do this by changing the domain, the quadratic form corresponding to the Laplacian is formally the same.

2.3.2. Definition. We define the Dirichlet Laplacian on $M$ by the quadratic form $q_{M}^{\mathrm{D}}(u)$ defined in Equation (2.14) for all $u \in \operatorname{dom} q_{M}^{\mathrm{D}}:=\mathscr{H}^{1}(M)$. Denote the corresponding operator by $\Delta_{M}^{\mathrm{D}}$

2.3.3. Definition. We define the Neumann Laplacian on $M$ by the quadratic form $q_{M}^{\mathrm{N}}(u)$ defined in Equation (2.14) for all $u \in \operatorname{dom} q_{M}^{\mathrm{N}}:=\mathscr{H}^{1}(M)$. Denote the corresponding operator by $\Delta_{M}^{\mathrm{N}}$ 
Note that the form norms $\|\cdot\|_{q_{M}^{\mathrm{D}}}$ and $\|\cdot\|_{q_{M}^{\mathrm{N}}}$ are equivalent to the first Sobolev norm $\|\cdot\|_{\mathscr{H}^{1}(M)}$. Remember that $\mathscr{H}^{1}(M)$ is the completion of $C^{\infty}(M)$ and that $\mathscr{\mathscr { H }}^{1}(M)$ is the closure of $C_{c}^{\infty}(M)$, the space of smooth functions with compact support away form $\partial M$.

It is clear that we can define mixed boundary conditions. Let $Z$ be a component of $\partial M$. Let $C_{Z}^{\infty}(M)$ denote the space of smooth functions with compact support away from $Z$. Thus $C_{c}^{\infty}(M)=C_{\partial M}^{\infty}(M)$. We define a quadratic form by (2.14) on the $\mathscr{H}^{1}$-closure of $C_{Z}^{\infty}(M)$. We call the corresponding Laplacian the Dirichlet-Neumann Laplacian (satisfying Dirichlet boundary conditions on $Z$ and Neumann boundary conditions on $\partial M \backslash Z$ ). In particular, if $M$ is the disjoint union of two manifolds $M^{1}$ and $M^{2}$ we denote the corresponding quadratic form with Dirichlet boundary conditions on $\partial M^{1}$ and Neumann boundary conditions on $\partial M^{2}$ by $q_{M^{1} \cup M^{2}}^{\mathrm{DN}}$. Similar notations like $q_{M^{1} \cup M^{2}}^{\mathrm{ND}}$ or $q_{M^{1} \cup M^{2}}^{\mathrm{DD}}$ are understood in an obvious manner.

In the next lemma, the eigenvalues are written in increasing order and repeated according to multiplicity as assumed in Section 1.3.

2.3.4. Lemma. $\quad 1$. Let $M$ be a compact manifold with boundary $\partial M$. Let $M^{\prime}$ be a submanifold of $M$ of the same dimension. Then the spectra of $\Delta_{M^{\prime}}^{\mathrm{D}}$ and $\Delta_{M}^{\mathrm{N}}$ are purely discrete and the eigenvalues satisfy

$$
\lambda_{k}^{\mathrm{N}}(M) \leq \lambda_{k}^{\mathrm{D}}\left(M^{\prime}\right)
$$

2. Suppose further that $M=M_{1} \cup M_{2}$ such that $M_{1} \cap M_{2}$ has measure 0 . Denote by $\lambda_{k}^{\mathrm{N}}\left(M_{1} \dot{\cup} M_{2}\right)$ and $\lambda_{k}^{\mathrm{D}}\left(M_{1} \dot{\cup} M_{2}\right)$ the eigenvalues of the quadratic forms defined on $\mathscr{H}^{1}\left(M_{1}\right) \oplus \mathscr{H}^{1}\left(M_{2}\right)$ and $\dot{\mathscr{H}}^{1}\left(M_{1}\right) \oplus \dot{\mathscr{H}}^{1}\left(M_{2}\right)$. Then we have

$$
\lambda_{k}^{\mathrm{N}}\left(M_{1} \dot{\cup} M_{2}\right) \leq \lambda_{k}^{\mathrm{N}}(M) \leq \lambda_{k}^{\mathrm{D}}(M) \leq \lambda_{k}^{\mathrm{D}}\left(M_{1} \dot{\cup} M_{2}\right) .
$$

This estimate is called Dirichlet-Neumann bracketing.

Proof. Theorem 2.2.4 ensures that the resolvent $\left(\Delta_{M}^{\mathrm{N}}+1\right)^{-1}$ is compact (see Lemma 1.3.2, note that $\mathscr{H}^{1}(M)$ and $\operatorname{dom} q_{M}^{\mathrm{N}}$ have equivalent norms). Therefore $\Delta_{M}^{\mathrm{N}}$ has purely discrete spectrum. By construction we have $\mathscr{H}^{1}\left(M^{\prime}\right) \subset \mathscr{H}^{1}(M)$. The Min-max Principle (Corollary 1.3.5) implies that $\Delta_{M^{\prime}}^{\mathrm{D}}$ has also purely discrete spectrum and that Inequality (2.15) is satisfied. Inequality (2.16) follows from the inclusions (resp. embedding maps by obvious identifications)

$$
\mathscr{\mathscr { H }}^{1}\left(M_{1}\right) \oplus \mathscr{H}^{1}\left(M_{2}\right) \subset \mathscr{H}^{1}(M) \subset \mathscr{H}^{1}(M) \subset \mathscr{H}^{1}\left(M_{1}\right) \oplus \mathscr{H}^{1}\left(M_{2}\right)
$$

in the same way.

Note that the eigenvalue 0 corresponds to functions constant on every component. If $M$ is connected the eigenspace corresponding to the eigenvalue 0 has dimension at most 1 , but the constant lies only in the domain of the Neumann Laplacian (quadratic form). Thus $\lambda_{1}^{\mathrm{N}}(M)=0$, $\lambda_{k}^{\mathrm{N}}(M)>0$ for $k \geq 2$ and $\lambda_{k}^{\mathrm{D}}(M)>0$ for $k \in \mathbb{N}$.

Since the Laplacians have purely discrete spectrum there exists an orthonormal basis consisting of eigenfunctions. The following result justifies the names "Dirichlet" and "Neumann" Laplacian (see [Tay96, Chapter 5] or [Hör85, Section 20.1].

2.3.5. Theorem. The eigenfunctions $\varphi$ of $\Delta_{M}^{\mathrm{D}}$ and $\Delta_{M}^{\mathrm{N}}$ belong to $C^{\infty}(M)$ and satisfy $\varphi \uparrow_{\partial M}=0$ resp. $\partial_{\mathrm{n}} \varphi \uparrow_{\partial M}=0$. 
Here we have set $\partial_{\mathrm{n}} u:=\mathrm{d} u(\vec{n})$ on $\partial M$ where $\vec{n}$ denotes the outward normal unit vector field of $\partial M$. Furthermore, denote by $v$ the corresponding 1-form defined on a neighbourhood of $\partial M$. Furthermore, denote by $l_{v}$ the interior product given by

$$
\left\langle u, \imath_{v} v\right\rangle=\langle v \wedge u, v\rangle
$$

i.e., $l_{v}$ is the formal adjoint of the exterior product $u \mapsto v \wedge u$. A proof of the following standard result can be found in [Tay96, Section 2.10].

2.3.6. Lemma (Gauss-Green Formula). Let $M$ be a Riemannian manifold with (piecewise) smooth boundary $\partial M$. Then we have

$$
\begin{gathered}
\langle\mathrm{d} u, \mathrm{~d} v\rangle+\left\langle\mathrm{d}^{*} u, \mathrm{~d}^{*} v\right\rangle=\left\langle\Delta_{\mathbf{A}^{p} T M} u, v\right\rangle+\int_{\partial M}\left(\langle\mathrm{~d} u, v \wedge v\rangle-\left\langle\mathrm{d}^{*} u, \imath_{v} v\right\rangle\right) \\
\langle\mathrm{d} u, \mathrm{~d} v\rangle=\left\langle\Delta_{M} u, v\right\rangle+\int_{\partial M} \partial_{\mathrm{n}} u \bar{v}
\end{gathered}
$$

for all $u \in \mathscr{H}^{2}\left(\mathbf{A}^{p} T M\right)$ and $v \in \mathscr{H}^{1}\left(\mathbf{A}^{p} T M\right)$ resp. $u \in \mathscr{H}^{2}(M)$ and $v \in \mathscr{H}^{1}(M)$.

Note that $\mathrm{d} u$ and $v$ can be restricted to $\partial M$ (see Theorem 2.2.5).

Now we introduce the Hodge duality operator (see e.g., [Tay96, Section 5.8]). We still assume that $M$ is orientable. By $\omega \in C^{\infty}\left(\mathbf{A}^{d} T M\right)$ we denote the volume form of the Riemannian manifold $M$. Let

$$
*: C^{\infty}\left(\mathbf{A}^{p} T M\right) \longrightarrow C^{\infty}\left(\mathbf{A}^{d-p} T M\right)
$$

be the Hodge duality operator characterised by

$$
u \wedge * v=\langle u, v\rangle_{\mathbf{A}^{p} T M} \omega
$$

for all $u, v \in C^{\infty}\left(\mathbf{A}^{p} T M\right)$. Since $* * u=(-1)^{p(d-p)} u$ on $p$-forms, $*$ is bijective. Furthermore, $*$ extends to a bounded operator onto the corresponding $L_{2}$-spaces. Here, we do not want to specify boundary conditions on $p$-forms (except for $\theta$-periodic boundary conditions in Section 3.4). Therefore, we suppose that $\partial M=\emptyset$. For the definition of natural boundary conditions on $p$-forms see e.g. [Tay96, Section 5.9].

2.3.7. Lemma. The *-operator commutes with the Laplacian, i.e.,

$$
\Delta_{\mathbf{A}^{d-p} T M} * u=* \Delta_{\mathbf{A}^{p} T M} u
$$

for all $u \in \mathscr{H}^{2}\left(\mathbf{A}^{p} T M\right)$.

We can apply the Hodge $*$-operator to obtain the following result:

2.3.8. Theorem. Suppose that $M$ is an orientable Riemannian manifold of dimension d (without boundary). Then we have the equality $\operatorname{dim}_{I}\left(\Delta_{\mathbf{A}^{p} T M}\right)=\operatorname{dim}_{I}\left(\Delta_{\mathbf{A}^{d-p} T M}\right)$ for all measurable sets $I \subset[0, \infty[$ and all $0 \leq p \leq d$. In particular,

$$
\operatorname{spec} \Delta_{\mathbf{A}^{p} T M}=\operatorname{spec} \Delta_{\mathbf{A}^{d-p} T M}
$$

If $M$ is compact then $\lambda_{k}\left(\mathbf{A}^{p} T M\right)=\lambda_{k}\left(\mathbf{A}^{d-p} T M\right)$ for all $k \in \mathbb{N}$. 
Proof. Denote by $E_{I}(H)$ the spectral projection of $H$ on $I$. By Lemma 2.3.7 we also have

$$
E_{I}\left(\Delta_{\mathbf{A}^{d-p} T M}\right) *=* E_{I}\left(\Delta_{\mathbf{A}^{p} T M}\right) .
$$

Since $*$ is bijective we conclude the first statement. The other statements follow.

Set $\mathbf{A}^{+} T M:=\bigoplus_{p \text { even }} \mathbf{A}^{p} T M$ and $\mathbf{A}^{-} T M:=\bigoplus_{p \text { odd }} \mathbf{A}^{p} T M$. We use supersymmetry to prove the following:

2.3.9. Theorem. Suppose that $\partial M=\emptyset$. Then we have $\operatorname{dim}_{I}\left(\Delta_{\mathbf{A}^{+} T M}\right)=\operatorname{dim}_{I}\left(\Delta_{\mathbf{A}^{-} T M}\right)$ for all measurable sets $I \subset] 0, \infty[$. In particular,

$$
\operatorname{spec} \Delta_{\mathbf{A}^{+} T M} \backslash\{0\}=\operatorname{spec} \Delta_{\mathbf{A}^{-} T M} \backslash\{0\} .
$$

Proof. Let $\mathscr{H}_{ \pm}:=L_{2}\left(\mathbf{A}^{ \pm} T M\right), Q:=\mathrm{d}+\mathrm{d}^{*}$. Then $H=Q^{2}=\Delta_{\mathbf{A} T M}$. Set $P u:= \pm u$ if $u \in \mathscr{H}_{ \pm}$. Furthermore, if $u \in \mathscr{H}_{ \pm}$, then $Q u \in \mathscr{H}_{\mp}$ and $P Q u=\mp Q u=-Q P u$. This proves that $(H, P, Q)$ has supersymmetry. The result follows by Theorem 1.3.10.

In dimension 2 the spectrum of the Laplacian on forms is completely determined by the spectrum of the Laplacian on functions:

2.3.10. Corollary. If $M^{\text {per }}$ is of dimension $d=2$ (without boundary) then we have $\operatorname{dim}_{I}\left(\Delta_{M}\right)=$ $\operatorname{dim}_{I}\left(\Delta_{\mathbf{A}^{1} T M}\right)=\operatorname{dim}_{I}\left(\Delta_{\mathbf{A}^{2} T M}\right)$ for any measurable set $\left.I \subset\right] 0, \infty[$. In particular,

$$
\operatorname{spec} \Delta_{M} \backslash\{0\}=\operatorname{spec} \Delta_{\mathbf{A}^{1} T M} \backslash\{0\}=\operatorname{spec} \Delta_{\mathbf{A}^{2} T M} \backslash\{0\} .
$$

Proof. The corollary follows directly from the preceding two theorems. Note that in dimension $2, \mathbf{A}^{+} T M=\mathbf{A}^{0} T M \oplus \mathbf{A}^{2} T M$ and $\mathbf{A}^{-} T M=\mathbf{A}^{1} T M$.

The next lemma shows that harmonic functions minimize the energy integral in the following sense:

2.3.11. Lemma. Let $M$ be a Riemannian manifold. Let $f$ be a Dirichlet function, i.e., $f \in$ $\ddot{\mathscr{H}}^{1}(M)$, and let $h \in C^{\infty}(M)$ be harmonic, i.e., $\Delta_{M} h=0$. Then we have $\langle\mathrm{d} h, \mathrm{~d} f\rangle_{L_{2}\left(T^{*} M\right)}=0$. In particular, we have

$$
\|\mathrm{d} h\|_{L_{2}\left(T^{*} M\right)}^{2} \leq\|\mathrm{d}(f+h)\|_{L_{2}\left(T^{*} M\right)}^{2} \quad \text { and } \quad\|\mathrm{d} f\|_{L_{2}\left(T^{*} M\right)}^{2} \leq\|\mathrm{d}(f+h)\|_{L_{2}\left(T^{*} M\right)}^{2} .
$$

Proof. Using the Gauss-Green Formula (2.18) with $f \in C_{c}^{\infty}(M)$, we obtain

$$
\langle\mathrm{d} h, \mathrm{~d} f\rangle_{L_{2}\left(T^{*} M\right)}=\langle\Delta h, f\rangle_{L_{2}(M)}=0 .
$$

A limiting argument shows that this is true for all $f \in \stackrel{\mathscr{H}}{ }^{1}(M)$.

The next result follows from elliptic regularity theory (see [Tay96, Chapter 5])

2.3.12. Lemma (Weyl). Suppose $h \in L_{2}(M)$ satisfies

$$
\int_{M} h \overline{\Delta v}=0
$$

for all $v \in C_{c}^{\infty}(M)$. Then $h \in C^{\infty}(\stackrel{\circ}{M})$ and $\Delta h=0$. 
Finally we quote another result due to Weyl. A proof can be found in [SV98, Theorem 1.2.1]. For the definition of the eigenvalue counting function see Definition 1.3.6.

2.3.13. Theorem (Weyl asymptotic). Let $M$ be a Riemannian manifold and denote by $\Delta_{M}^{\mathrm{D} / \mathrm{N}}$ the corresponding Laplacian with arbitrary boundary conditions (if $\partial M \neq \emptyset$ ). Then we have

$$
\operatorname{dim}_{\lambda}\left(\Delta_{M}^{\mathrm{D} / \mathrm{N}}\right)-\frac{\operatorname{vol}\left(\mathbb{B}^{d}\right)}{(2 \pi)^{d}} \operatorname{vol}(M) \lambda^{\frac{d}{2}}=O\left(\lambda^{\frac{d-1}{2}}\right)
$$

as $\lambda \rightarrow \infty$. Here, $\mathbb{B}^{d}:=\left\{x \in \mathbb{R}^{d}|| x \mid \leq 1\right\}$ denotes the unit ball in $\mathbb{R}^{d}$.

Physically, the asymptotic behaviour of the eigenvalue counting function can be motivated in the following way (see [RS78, Section XXX.15]):

2.3.14. Remark. If $\Delta_{M}^{\mathrm{D} / \mathrm{N}}$ describes the energy of a quantum mechanical system (with normalized mass and units such that the Planck constant satisfies $\mathrm{h}=1)$ then $\operatorname{dim}_{\lambda}\left(\Delta_{M}^{\mathrm{D} / \mathrm{N}}\right)$ is the number of states with energy not greater than $\lambda$. Therefore we can understand the Weyl asymptotic in a semi-classical picture: In classical mechanics, the energy level $\lambda$ determines a certain allowed region in the phase space $T^{*} M$ with respect to the Hamiltonian $h(x, \xi)=g_{x}(\xi, \xi)$, $(x, \xi) \in T^{*} M$ where $g$ denotes the Riemannian metric on $T^{*} M$. Here, an arbitrary number of particles could be accomodated in this region of volume

$$
\operatorname{vol}_{T^{*} M}\left\{(x, \xi) \in T^{*} M \mid h(x, \xi) \leq \lambda\right\}=\lambda^{d / 2} \operatorname{vol}\left(\mathbb{B}^{d}\right) \operatorname{vol} M .
$$

In quantum mechanics, however, the uncertainty principle implies that any particle occupies a volume $(2 \pi \mathrm{h})^{d}$. Therefore the number of states of the corresponding Hamiltonian $\Delta_{M}$ in quantum mechanics is approximately the allowed volume in phase space devided by $(2 \pi \mathrm{h})^{d}=$ $(2 \pi)^{d}$ in our units.

\subsection{Metric perturbations}

In this section, we give some results on how to deal with Riemannian manifolds with changing metric. Some of the results here can be found in standard text books, but we need an explicit control on the constants in the estimates. This is necessary since in the application in Chapter 8 we deal with an increasing sequence of manifolds and we need bounds independent of this sequence.

To construct perturbations of the metric on the manifold $M$ we define a norm on $C^{k}\left(\mathbf{S}^{2} T M\right)$. Here, $C^{k}\left(\mathbf{S}^{2} T M\right)$ denotes the space of $C^{k}$-sections in the bundle of bilinear symmetric forms $\mathbf{S}^{2} T M$, i.e., the fibers $\mathbf{S}^{2} T M_{x}=\mathbf{S}^{2} T_{x} M$ consist of all bilinear symmetric forms on $T_{x} M$. Therefore, a Riemannian metric $g$ is a section in $\mathbf{S}^{2} T M$ such that $g_{x}$ is strictly positive definite for all $x \in M$.

2.4.1. Definition. A Riemannian metric $g$ on $M$ will be called $C^{k}$-bounded (with respect to the $C^{k+1}$-bounded atlas $\left.\mathscr{A}=\left(\varphi_{\alpha}: U_{\alpha} \longrightarrow V_{\alpha}\right)_{\alpha \in A}\right)$ if

$$
\|g\|_{C^{k}(M)}:=\|g\|_{C^{k}(M), \mathscr{A}}:=\sup _{\alpha \in A}\left\|g_{\alpha}\right\|_{C^{k}\left(V_{\alpha}\right)}<\infty
$$

where $g_{\alpha}=\left(g_{\alpha, i j}\right)$ denotes the matrix-valued local representation of $g$ in the chart $U_{\alpha}$. 
Note that this definition is independent of the choice of the atlas $\mathscr{A}$. If we choose another $C^{k+1}$-bounded atlas $\widetilde{\mathscr{A}}$ then there exists a constant $c=c\left(c_{0}\right) \geq 1$ such that

$$
\frac{1}{c}\|g\|_{C^{k}(M), \mathscr{A}} \leq\|g\|_{\left.C^{k}(M), \widetilde{A}\right)} \leq c\|g\|_{C^{k}(M), \mathscr{A}}
$$

for all $g \in C^{k}\left(\mathbf{S}^{2} T M\right)$. This is true because all transition maps have the uniform $C^{k+1}$-bound $c_{0}$. Note that we need the derivative of $\varphi_{\alpha} \circ \varphi_{\alpha^{\prime}}^{-1}$ to transform $g_{\alpha}$ into $g_{\alpha^{\prime}}$.

The next definition is necessary in order to apply regularity theory. Let

$$
\underline{g}_{\alpha}(y):=\inf _{v \in \mathbb{R}^{d} \backslash\{0\}} \frac{g_{\alpha, y}(v, v)}{|v|^{2}} \quad \text { and } \quad \bar{g}_{\alpha}(y):=\sup _{v \in \mathbb{R}^{d} \backslash\{0\}} \frac{g_{\alpha, y}(v, v)}{|v|^{2}}
$$

be the smallest resp. greatest eigenvalue of the local representation $g_{\alpha}$ of $g$ at the point $y \in V_{\alpha}$, i.e., the smallest resp. geratest eigenvalue of the matrix $\left(g_{\alpha, i j}(y)\right)_{i j}$.

2.4.2. Definition. We say that $g$ is uniformly elliptic with respect to the altlas $\mathscr{A}$ if there exist constants $0<\underline{g}_{0} \leq \bar{g}_{0}<\infty$ such that

$$
\underline{g}_{0} \leq \inf _{\alpha \in A} \underline{g}_{\alpha}(y) \leq \inf _{\alpha \in A} \bar{g}_{\alpha}(y) \leq \bar{g}_{0}
$$

for all $y \in M$. We briefly write $\underline{g}_{0} \leq g \leq \bar{g}_{0}$ (with respect to $\mathscr{A}$ ).

Note that on a compact manifold, every metric $g$ on $M$ is uniformly elliptic with respect to a suitable atlas $\mathscr{A}$.

There are two useful tools when dealing with estimates. Let $E$ and $F$ be normed vector spaces.

2.4.3. Lemma. Let $D$ be an open subset of $E \times E$ such that $(x, x) \in D$ for all $x \in E$. Suppose that $R: D \longrightarrow F$ satisfies $R(x, x)=0$. Then the following statements are equivalent:

1. The map $R$ is uniformly continuous on a neighbourhood of the diagonal $\{(x, x) \mid x \in D\}$.

2. There exists a monotone increasing function $\eta(\delta) \rightarrow 0$ as $\delta \rightarrow 0$ such that $\|R(x, y)\|_{F} \leq$ $\eta\left(\|x-y\|_{E}\right)$ for all $(x, y) \in D$.

In particular, a function $f: U \longrightarrow F$ defined on some open subset $U$ of $E$ is uniformly continuous if and only if there exists a monotone increasing function $\eta(\delta) \rightarrow 0$ as $\delta \rightarrow 0$ such that $\|f(x)-f(y)\| \leq \eta(\|x-y\|)$.

Proof. The function $\eta$ can be defined by

$$
\eta(\delta):=\sup \left\{\|R(x, y)\|_{F} \mid(x, y) \in D,\|x-y\|_{E} \leq \delta\right\} .
$$

Clearly, $\eta$ is monotone. The uniform continuity of $R$ implies the continuity of $\eta$.

2.4.4. Lemma. Let $f: M \longrightarrow F$ be continuous in $x_{0} \in M \subset E$. Then there exists a monotone increasing function $c(\delta)$ such that $\|f(x)\| \leq c(\|x\|)$ for all $x \in M$.

Note that $c\left(\delta_{n}\right)$ is a bounded sequence if $\left(\delta_{n}\right)$ is bounded. 
Proof. We set

$$
\eta(\delta):=\sup \left\{\left|f(x)-f\left(x_{0}\right)\right||| x-x_{0} \mid \leq \delta, x \in M\right\} .
$$

Since $f$ is continuous in $x_{0}, \eta(\delta)<\infty$. Furthermore, from the definition and the monotonicity of $\eta(\delta)$ we obtain

$$
|f(x)| \leq\left|f\left(x_{0}\right)\right|+\eta\left(|x|+\left|x_{0}\right|\right)=: c(|x|) .
$$

Note that $c$ depends on $f$ and $x_{0} \in M$.

2.4.5. Remark. We will apply this lemma in the following situation: Let $V$ be open in $\mathbb{R}^{d}$ and let $G: V \longrightarrow L\left(\mathbb{R}^{d}\right)$ be a $C^{k}$-map. Suppose further that $f$ is a continuous function in $G$. We need a fixed space $E$ independent of the open set $V$. This can be achieved by extending the map $G$ to a map $\hat{G}: \mathbb{R}^{d} \longrightarrow L\left(\mathbb{R}^{d}\right)$ with $\|\hat{G}\|_{C^{k}} \leq e_{0}\|G\|_{C^{k}}$. Here, $e_{0}$ is a constant depending on $\partial V, d$ and $k$ (see [Ste70]). But we can choose an atlas which has only a finite number of different shapes $\partial V_{\alpha}$ by restricting the charts to smaller sets if necessary. Therefore, we can assume that $e_{0}$ is independent on $V$. Applying Lemma 2.4.4 with $E:=C^{k}\left(\mathbb{R}^{d}, \mathbf{L}\left(\mathbb{R}^{d}\right)\right)$ we obtain a monotone increasing function $c(\delta)$ independent of $V$ such that $\|f(G)\| \leq\|f(\hat{G})\| \leq c(\|\hat{G}\|) \leq c\left(e_{0}\|G\|\right)$.

From now on, we assume that there exists a $C^{2}$-bounded atlas $\mathscr{A}=\left(\varphi_{\alpha}: U_{\alpha} \longrightarrow V_{\alpha}\right)$ with subordinate $C^{2}$-bounded partition of unity $\left(\chi_{\alpha}\right)$ with bound $c_{0}$, distance from the boundary $d_{0}>0$ and maximal number $N$ of neighbours (see Definition 2.2.1). The assumption $0<\underline{g}_{0} \leq$ $g \leq \bar{g}_{0}<\infty$ always refers to the atlas $\mathscr{A}$ (cf. Definition 2.4.2). The existence of such metrics $g$ and atlases $\mathscr{A}$ will be proven in Section 3.6.

We always assume that $U$ and $V$ of a chart $\varphi: U \longrightarrow V$ are relatively compact. Note that we switch without mentioning between a function on $U$ and on $V$. On $U$, we always use the definition of the corresponding function space defined for manifolds, on $V$ we use the usual definition on $V \subset \mathbb{R}_{+}^{d}$. Furthermore, we only allow such sets $V$ for which an extension map of $C^{k}$-maps on $V$ to $C^{k}$-maps on $\mathbb{R}^{d}$ with norm smaller than $e_{0}$ exists (see Remark 2.4.5). Here, $e_{0}$ is a constant independent on $V$.

Let $M$ resp. $\widetilde{M}$ be the Riemannian manifold $M$ with metric $g$ resp. $\widetilde{g}$. Similarly, for an open subset $U \subset M$, we endow $U$ with the metric $g$ and $\widetilde{U}$ with the metric $\widetilde{g}$. Sometimes it is convenient to write

$$
|\mathrm{d} u|_{T^{*} U}^{2}=\sum_{i, j=1}^{d} g^{i j} \partial_{i} u \overline{\partial_{j} u}=\left\langle G^{-1} \nabla u, \nabla u\right\rangle_{\mathbb{R}^{d}}
$$

with $G: V \longrightarrow \mathbf{L}\left(\mathbb{R}^{d}\right)$. Here, $G(y)$ denotes the strictly positive linear map associated with the matrix $\left(g_{i j}(y)\right)_{i j}$ for $y \in V$.

In the sequel, the various functions $\eta, \eta_{i}$ and $c, c_{i}$ depend only on $\underline{g}_{0}, \bar{g}_{0}, c_{0}, d_{0}, e_{0}$ and $N$. The following lemma gives estimates for the $L_{2}$-norm and the quadratic form of the Laplacian defined by different metrics on $M$.

2.4.6. Lemma. For all constants $0<\underline{g}_{0} \leq \bar{g}_{0}<\infty$ there exists a monotone increasing function $\eta(\delta) \rightarrow 0$ as $\delta \rightarrow 0$ such that

$$
\begin{aligned}
\left|\|u\|_{L_{2}(M)}^{2}-\|u\|_{L_{2}(\widetilde{M})}^{2}\right| & \leq \eta\left(\|g-\widetilde{g}\|_{C^{0}(M)}\right)\|u\|_{L_{2}(M)}^{2} \\
\left|\|\mathrm{~d} u\|_{L_{2}\left(T^{*} M\right)}^{2}-\|\mathrm{d} u\|_{L_{2}\left(T^{*} \widetilde{M}\right)}^{2}\right| & \leq \eta\left(\|g-\widetilde{g}\|_{C^{0}(M)}\right)\|\mathrm{d} u\|_{L_{2}\left(T^{*} M\right)}^{2}
\end{aligned}
$$


for all $u \in L_{2}(M)$ resp. $u \in \mathscr{H}^{1}(M)$, all metrics $g$ and $\widetilde{g}$ on $M$ such that $\underline{g}_{0} \leq g, \tilde{g} \leq \bar{g}_{0}$, and all manifolds $M$.

Proof. We only prove (2.25) since the proof of (2.24) is similar but simpler. By Equation (2.23) we have

$$
\int_{M}|\mathrm{~d} u|^{2}=\sum_{\alpha} \int_{V_{\alpha}} \chi_{\alpha}\left\langle G_{\alpha}^{-1} \nabla u, \nabla u\right\rangle_{\mathbb{R}^{d}}\left(\operatorname{det} G_{\alpha}\right)^{\frac{1}{2}}
$$

for all $u \in \mathscr{H}^{1}(M)$, and a similar remark holds for $\widetilde{G}_{\alpha}$. Now we estimate

$$
\begin{aligned}
\left.\left|\int_{M}\right| \mathrm{d} u\right|^{2}-\int_{\widetilde{M}}|\mathrm{~d} u|^{2} \mid & \leq \\
\leq \sum_{\alpha} \int_{V_{\alpha}} \chi_{\alpha} \mid & \left\langle\left(\mathbb{1}-\left(\frac{\operatorname{det} \widetilde{G}_{\alpha}}{\operatorname{det} G_{\alpha}}\right)^{\frac{1}{2}} G_{\alpha}^{\frac{1}{2}} \widetilde{G}_{\alpha}^{-1} G_{\alpha}^{\frac{1}{2}}\right) G_{\alpha}^{-\frac{1}{2}} \nabla u, G_{\alpha}^{-\frac{1}{2}} \nabla u\right\rangle \mid\left(\operatorname{det} G_{\alpha}\right)^{\frac{1}{2}} \\
& \leq \sup _{\alpha} \sup _{y \in V_{\alpha}}\left|R\left(G_{\alpha}(y), \widetilde{G}_{\alpha}(y)\right)\right|_{\mathbf{L}\left(\mathbb{R}^{d}\right)} \sum_{\alpha} \int_{V_{\alpha}} \chi_{\alpha}\left\langle G_{\alpha}^{-1} \nabla u, \nabla u\right\rangle\left(\operatorname{det} G_{\alpha}\right)^{\frac{1}{2}} .
\end{aligned}
$$

Here,

$$
R(G, \widetilde{G}):=\mathbb{1}-(\operatorname{det} \widetilde{G} / \operatorname{det} G)^{\frac{1}{2}} G^{\frac{1}{2}} \widetilde{G}^{-1} G^{\frac{1}{2}}
$$

for any two matrices $G$ and $\widetilde{G}$ is a continuous (matrix-valued) function on the compact set $D:=\left\{\left.(G, \widetilde{G})\right|_{g_{0}} \leq|G|_{\mathbf{L}\left(\mathbb{R}^{d}\right)},|\widetilde{G}|_{\mathbf{L}\left(\mathbb{R}^{d}\right)} \leq \bar{g}_{0}\right\}$ and therefore uniformly continuous. Since furthermore $R(G, G)=0$ there exists a monotone function $\eta(\delta) \rightarrow 0$ as $\delta \rightarrow 0$ (see Lemma 2.4.3) such that

$$
|R(G, \widetilde{G})|_{\mathbf{L}\left(\mathbb{R}^{d}\right)} \leq \eta\left(|G-\widetilde{G}|_{\mathbb{R}^{d^{2}}}\right)
$$

for all $(G, \widetilde{G}) \in D$ where we have chosen the equivalent norm $|G|_{\mathbb{R}^{d^{2}}}:=\sup _{i, j}\left|G_{i j}\right|$ on $E=$ $\mathbb{R}^{d^{2}} \cong \mathbf{L}\left(\mathbb{R}^{d}\right)$. Finally, we have

$$
\sup _{\alpha} \sup _{y \in V_{\alpha}} \eta\left(\left|R\left(G_{\alpha}(y), \widetilde{G}_{\alpha}(y)\right)\right|_{\mathbf{L}\left(\mathbb{R}^{d}\right)}\right) \leq \eta\left(\|g-\widetilde{g}\|_{C^{0}(M)}\right)
$$

which ends our proof.

In the rest of this section we give (among other estimates) a similar result for the difference of two Laplacians arising from different metrics.

Note that we have to localize the global functions. We cannot quote standard arguments here since we need bounds independent of the manifold $M$. In our application, $M=M^{n}$ will be an increasing sequence of compact manifolds converging to a non-compact manifold. Sometimes, we even need to apply the results with $M$ non-compact (see Theorem 8.1.7).

2.4.7. Lemma. For all constants $0<g_{0} \leq \bar{g}_{0}<\infty$ there exist monotone increasing functions $c_{1}(\delta)$ and $\eta_{1}(\delta) \rightarrow 0$ as $\delta \rightarrow 0$ such that

$$
\begin{aligned}
\left\|\Delta_{M} u\right\|_{L_{2}(M)}^{2} & \leq c_{1}\left(\|g\|_{C^{1}(M)}\right)\|u\|_{\mathscr{H}^{2}(M)}^{2} \\
\left\|\left(\Delta_{M}-\Delta_{\widetilde{M}}\right) u\right\|_{L_{2}(M)}^{2} & \leq \eta_{1}\left(\|g-\widetilde{g}\|_{C^{1}(M)}\right)\|u\|_{\mathscr{H}^{2}(M)}^{2}
\end{aligned}
$$

for all $u \in \mathscr{H}^{2}(M)$, all metrics $g$ and $\widetilde{g}$ on $U$ such that $\underline{g}_{0} \leq g, \widetilde{g} \leq \bar{g}_{0}$, and all manifolds $M$. 
Proof. First, we prove the local estimates in the chart $\varphi_{\alpha}: U_{\alpha} \longrightarrow V_{\alpha}$. The local proof of (2.26) is quite obvious. Applying Lemma 2.4 .4 we obtain a monotone increasing function $c_{1}^{\prime}(\delta)$ independent on $\alpha$ (see Remark 2.4.5).

The local proof of Estimate (2.27) is essentially the same as the proof of Lemma 2.4.6. Here, we have to estimate a continuous function $R$ depending on $g_{\alpha, i j}, \widetilde{g}_{\alpha, i j}, \partial_{k} g_{\alpha, i j}$ and $\partial_{k} \widetilde{g}_{\alpha, i j}$ which is 0 if $g_{\alpha}=\widetilde{g}_{\alpha}$. Again, since $g$ and $\widetilde{g}$ are uniformly elliptic, we can restrict the definition of $R$ to some compact set $D$. Therefore, a monotone function $\eta_{1}^{\prime}(\delta)$ exists by Lemma 2.4.3 such that the local Estimate (2.26) holds.

The step from local to global estimates is simple. Using Lemma 2.2.2 and the local version of (2.26) we obtain

$$
\begin{aligned}
&\left\|\Delta_{M} u\right\|^{2} \leq 2 N \sum_{\alpha}\left\|\Delta_{U_{\alpha}} u_{\alpha}\right\|^{2} \leq 2 N \sum_{\alpha} c_{1}^{\prime}\left(\|g\|_{C^{1}\left(U_{\alpha}\right)}\right)\left\|u_{\alpha}\right\|_{\mathscr{H}^{2}\left(U_{\alpha}\right)}^{2} \leq \\
& \leq 2 N c_{1}^{\prime}\left(\|g\|_{C^{1}(M)}^{2}\right)\|u\|_{\mathscr{H}^{2}(M, \mathscr{A})}^{2}
\end{aligned}
$$

where we have set $u_{\alpha}:=\chi_{\alpha} u$ (note that we have used the monotonicity of $c_{1}^{\prime}(\delta)$ ). Therefore $c_{1}(\delta):=2 N c_{1}^{\prime}(\delta)$ is sufficient. The global proof of (2.27) is similar.

Now we show that locally, the first order differential operator $\left[\Delta_{U}, \chi\right]$ can be estimated by a second order differential operator.

2.4.8. Lemma. For all constants $0<\underline{g}_{0} \leq \bar{g}_{0}<\infty$ there exists a monotone increasing function $c_{2}^{\prime}(\delta)$ such that

$$
\left\|\left[\Delta_{U}, \chi\right] u\right\|_{L_{2}(U)} \leq c_{2}^{\prime}\left(\|g\|_{C^{1}(U)}\right)\|\chi\|_{C^{1}(U)}\left(\|u\|_{L_{2}(U)}^{2}+\left\|\Delta_{U} u\right\|_{L_{2}(U)}^{2}\right)
$$

for all $u \in \mathscr{H}^{2}(U) \cap \stackrel{\mathscr{H}}{ }^{1}(U)$, all $\chi \in C^{\infty}(U)$, all metrics $g$ on $U$ such that $\underline{g}_{0} \leq g \leq \bar{g}_{0}$, and all charts $\varphi: U \longrightarrow V$.

Proof. Without loss of generality assume that $u \in C^{\infty}(\bar{U})$ with $u \uparrow_{\partial U}=0$. Again, $G(y)$ denotes the strictly positive linear map in $\mathbb{R}^{d}$ associated with the matrix $\left(g_{i j}(y)\right)_{i j}$ as in Equation (2.23). Since $T=\left[\Delta_{U}, \chi\right]$ is a first order differential operator we can write $T u=\sum_{i=1}^{d} t_{i} \partial_{i} u+t_{0} u$ with smooth coefficients $t=\left(t_{0}, \ldots, t_{d}\right)$. Denote by $\|t\|_{C^{0}(U)}$ the maximum of the supremum norms of $t_{i}$. Then we have

$$
\begin{aligned}
\|T u\|_{L_{2}(U)}^{2} & \leq 2 \int_{U}\left(\left|\sum_{i=1}^{d} t_{i} \partial_{i} u\right|^{2}+\left|t_{0} u\right|^{2}\right)(\operatorname{det} G)^{\frac{1}{2}} \\
& \leq 2 \int_{U}\langle A \nabla u, \nabla u\rangle(\operatorname{det} G)^{\frac{1}{2}}+2\left\|t_{0}\right\|_{C^{0}(U)}^{2}\|u\|_{L_{2}(U)}^{2}
\end{aligned}
$$

where $A(y)$ denotes the linear map represented by the matrix $\left(t_{i}(y) \overline{t_{j}(y)}\right)_{i j}$. Furthermore we estimate similarly as in the proof of Lemma 2.4.6

$$
\begin{aligned}
\int_{U}\langle A \nabla u, \nabla u\rangle(\operatorname{det} G)^{\frac{1}{2}} & \leq\|A G\|_{C^{0}\left(U, \mathbf{L}\left(\mathbb{R}^{d}\right)\right)} \int_{U}\left\langle G^{-1} \nabla u, \nabla u\right\rangle(\operatorname{det} G)^{\frac{1}{2}} \\
& \leq\|A\|_{C^{0}\left(U, \mathbf{L}\left(\mathbb{R}^{d}\right)\right)}\|G\|_{C^{0}\left(U, \mathbf{L}\left(\mathbb{R}^{d}\right)\right)}\left\langle\Delta_{U} u, u\right\rangle_{L_{2}(U)}
\end{aligned}
$$


where we have used the Gauss-Green Formula (2.18). Here, the boundary term vanishes since $u \Upsilon_{\partial U}=0$. By the Cauchy-Young Inequality (1.1) we obtain

$$
\left\langle\Delta_{U} u, u\right\rangle_{L_{2}(U)} \leq\left\|\Delta_{U} u\right\|_{L_{2}(U)}^{2}+\|u\|_{L_{2}(U)}^{2}
$$

By the construction of $A(y)$ we have $|A(y)|_{\mathbf{L}\left(\mathbb{R}^{d}\right)} \leq d \max _{i}\left|t_{i}(y)\right|$. Furthermore, $\|t\|_{C^{0}(U)}$ can be estimated by $c_{2}^{\prime \prime}\left(\|g\|_{C^{1}(U)}\right)\|\chi\|_{C^{1}(U)}$ for some monotone function $c_{2}^{\prime \prime}(\delta)$ (see Lemma 2.4.4 and Remark 2.4.5). Therefore we obtain

$$
\|T u\|_{L_{2}(U)}^{2} \leq f(G)\|t\|_{C^{0}(U)}\left(\left\|\Delta_{U} u\right\|_{L_{2}(U)}^{2}+\|u\|_{L_{2}(U)}^{2}\right)
$$

with the continuous function $f$ defined by

$$
f(G):=2 d\left(\|G\|_{C^{0}\left(\mathbb{R}^{d}, \mathbf{L}\left(\mathbb{R}^{d}\right)\right)}+1\right)
$$

Again, by applying Lemma 2.4.4 there exists $c_{2}^{\prime \prime \prime}(\delta)$ estimating $f(G)$. Therefore, we can set $c_{2}^{\prime}(\delta):=c_{2}^{\prime \prime}(\delta) c_{2}^{\prime \prime \prime}(\delta)$.

2.4.9. Lemma. For all constants $c_{0}>0,0<\underline{g}_{0} \leq \bar{g}_{0}<\infty$ there exists a monotone increasing function $c_{2}(\delta)$ such that

$$
\left\|\left[\Delta_{M}, \chi\right] u\right\|_{L_{2}(M)}^{2} \leq c_{2}\left(\|g\|_{C^{1}(M)}\right)\|\chi\|_{C^{1}(M)}\|u\|_{\mathscr{H}^{1}(M)}^{2}
$$

for all $u \in \mathscr{H}^{1}(M)$, all $\chi \in C^{\infty}(M)$, all metrics $g$ such that $\underline{g}_{0} \leq g \leq \bar{g}_{0}$, and all manifolds $M$.

Proof. Locally, this is clear since $\left[\Delta_{U_{\alpha}}, \chi_{\alpha} \chi\right]$ is a first order differential operator. In the global estimate we can set $c_{2}(\delta):=c_{0} c_{2}^{\prime \prime}(\delta)$ where $c_{2}^{\prime \prime}(\delta)$ is defined in the previous proof, and where $c_{0}$ is the $C^{2}$-bound of the partition of unity.

Finally, we quote results from regularity theory to obtain bounds on the $\mathscr{H}^{2}$-norm. Here, we need Assumption (2.10) on the partition of unity.

2.4.10. Theorem. For all constants $c_{0}, d_{0}>0,0<\underline{g}_{0} \leq \bar{g}_{0}<\infty$ there exists a monotone increasing function $c_{3}(\delta)$ such that

$$
\|u\|_{\mathscr{H}^{2}(M)}^{2} \leq c_{3}\left(\|g\|_{C^{1}(M)}\right)\left(\|u\|_{L_{2}(M)}^{2}+\left\|\Delta_{M} u\right\|_{L_{2}(M)}^{2}\right)
$$

for all $u \in \mathscr{H}^{2}(M) \cap \stackrel{\mathscr{H}}{ }^{1}(M)$, all metrics $g$ such that $\underline{g}_{0} \leq g \leq \bar{g}_{0}$, and all manifolds $M$ with smooth boundary $\partial M$.

Proof. The proof of the local estimate (with $U_{\alpha}$ and $u_{\alpha}=\chi_{\alpha} u$ instead of $M$ and $u$ ) is standard in elliptic regularity theory, see e. g. [GT77, Theorem 8.12] or [Tay96, Theorem 5.1.3]. The constant in Inequality (2.29) depends continuously on the coefficients $g_{\alpha}$ of the elliptic operator $\Delta_{U_{\alpha}}$ and its first derivatives. These coefficients are indeed controlled by $\left\|g_{\alpha}\right\|_{C^{1}\left(U_{\alpha}\right)}$. By Lemma 2.4.4 the desired function $c_{3}^{\prime}(\delta)$ in the local estimate exists (see also Remark 2.4.5). Note that we do not have a dependence on the boundary of $V_{\alpha}$ by Assumption (2.10). 
To obtain the global estimate we calculate

$$
\begin{aligned}
\|u\|_{\mathscr{H}^{2}(M), \mathscr{A}} & =\sum_{\alpha}\left\|u_{\alpha}\right\|_{\mathscr{H}^{2}\left(V_{\alpha}\right)}^{2} \\
& \leq c_{3}^{\prime}\left(\|g\|_{C^{1}}\right) \sum_{\alpha}\left(\left\|u_{\alpha}\right\|_{L_{2}\left(U_{\alpha}\right)}^{2}+\left\|\Delta_{U_{\alpha}} u_{\alpha}\right\|_{L_{2}\left(U_{\alpha}\right)}^{2}\right) \\
& \leq c_{3}^{\prime}\left(\|g\|_{C^{1}}\right)\left(\|u\|_{L_{2}(M)}^{2}+\left\|\Delta_{M} u\right\|_{L_{2}(M)}^{2}+\sum_{\alpha}\left\|\left[\Delta_{U_{\alpha}}, \chi_{\alpha}\right] u\right\|_{L_{2}(M)}^{2}\right)
\end{aligned}
$$

where we have used Lemma 2.2.2 and the monotonicity of $c_{3}^{\prime}(\delta)$. Applying Lemma 2.4.8 we obtain

$$
\left\|\left[\Delta_{U_{\alpha}}, \chi_{\alpha}\right] u\right\|^{2} \leq c_{2}^{\prime}\left(\left\|g_{\alpha}\right\|_{C^{1}}\right)\left\|\chi_{\alpha}\right\|_{C^{1}}\left(\|u\|_{L_{2}\left(U_{\alpha}\right)}^{2}+\left\|\Delta_{U_{\alpha}} u\right\|_{L_{2}\left(U_{\alpha}\right)}^{2}\right) .
$$

Finally, we can set $c_{3}(\delta):=c_{3}^{\prime}(\delta)\left(1+c_{0} c_{2}^{\prime}(\delta)\right)$. Here, we have applied Lemma 2.2.2 again.

\subsection{The Decomposition Principle}

In this section we prove that the essential spectrum is determined by the geometry at infinity, i.e., the essential spectrum remains invariant under compact perturbations of the manifold. This result will be needed in Chapter 8. A similar result for the continuous spectrum is proven in [DL79].

Suppose that $M$ is a manifold which admits an atlas with a finite number of neighbours and a $C^{2}$-bounded subordinate partition of unity with strictly positive distance from the boundary (see Definition 2.2.1). Furthermore, suppose that a $C^{1}$-bounded metric with respect to the atlas exists. Therefore the results of the last section can be applied.

Remember that $\lambda \in \operatorname{ess} \operatorname{spec} \Delta_{M}$ if and only if there exists a singular sequence for $\lambda$ and $\Delta_{M}$ (see Weyl's Criterion Lemma 1.3.1).

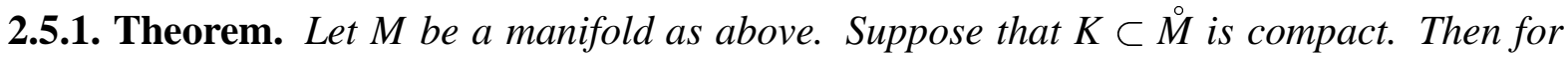
every $\lambda \in \operatorname{ess~} \operatorname{spec} \Delta_{M}$ there exists a singular sequence with support away from $K$.

Proof. Let $\left(u_{n}\right)$ be singular sequence for $\lambda$ and $\Delta_{M}$, i.e., $\left\|u_{n}\right\|=1, u_{n} \rightarrow 0$ weakly in $L_{2}(M)$ and $\left\|\left(\Delta_{M}-\lambda\right) u\right\| \rightarrow 0$. Let $U_{1}, U_{2}$ be open, relatively compact sets such that $K \subset U_{1} \subset \bar{U}_{1} \subset$

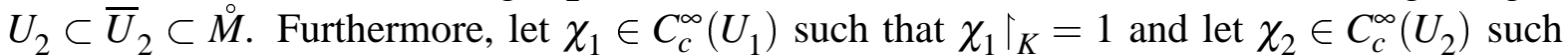
that $\chi_{2} \uparrow_{U 1}=1$. Since $\operatorname{dist}\left(\operatorname{supp} \chi_{2}, \partial M\right)>0$ we do not need that $\partial M$ is smooth, i.e., $\partial M$ could have singularities as corners etc. By Theorem 2.4.10 and Lemma 2.4.9 there exists a constant $c^{\prime}>0$ such that

$$
\begin{aligned}
\left\|\chi_{2} u_{n}\right\|_{\mathscr{H}^{2}\left(\bar{U}_{2}\right)}^{2} & \leq c^{\prime}\left(\left\|u_{n}\right\|_{L_{2}(M)}^{2}+\left\|\Delta_{M} u_{n}\right\|_{L_{2}(M)}^{2}\right) \\
& \leq c^{\prime}\left((1+\lambda)\left\|u_{n}\right\|_{L_{2}(M)}^{2}+\left\|\left(\Delta_{M}-\lambda\right) u_{n}\right\|_{L_{2}(M)}^{2}\right)
\end{aligned}
$$

for all $n \in \mathbb{N}$. Therefore $\left(\chi_{2} u_{n}\right)$ is bounded in $\mathscr{H}^{2}\left(\bar{U}_{2}\right)$. By passing to a subsequence also denoted by $\left(\chi_{2} u_{n}\right)$ we can assume that $\chi_{2} u_{n} \rightarrow 0$ weakly in $\mathscr{H}^{2}\left(\bar{U}_{2}\right)$ (Theorem 1.1.1). By the Rellich-Kondrachov Compactness Theorem (Theorem 2.2.4) we have $\chi_{n} u_{n} \rightarrow 0$ in norm in $\mathscr{H}^{1}\left(\bar{U}_{2}\right)$, in particular, $\chi_{2} u_{n} \rightarrow 0$ in $L_{2}\left(\bar{U}_{2}\right)$. 
Let $\widetilde{v}_{n}:=\left(1-\chi_{1}\right) u_{n}$. Since we have $1=\left\|u_{n}\right\| \leq\left\|\chi_{2} u_{n}\right\|+\left\|\widetilde{v}_{n}\right\|$ there exists a constant $c^{\prime \prime}>0$ such that $\left\|\widetilde{v}_{n}\right\| \geq 1 / c^{\prime \prime}$. Now we can show that $v_{n}:=\widetilde{v}_{n} /\left\|\widetilde{v}_{n}\right\|$ is the desired singular sequence. Clearly, $\operatorname{supp} v_{n} \subset M \backslash K$ and $v_{n} \rightarrow 0$ weakly in $L_{2}(M)$ by the existence of $c^{\prime \prime}$. Finally, note that

$$
\left\|\left(\Delta_{M}-\lambda\right) v_{n}\right\|_{L_{2}(M)} \leq c^{\prime \prime}\left(\left\|\left(\Delta_{M}-\lambda\right) u_{n}\right\|_{L_{2}(M)}+\left\|\left[\Delta_{M}, \chi_{1}\right] u_{n}\right\|_{L_{2}\left(\bar{U}_{1}\right)}\right) .
$$

The first term converges to 0 by our assumption on $u_{n}$, the second term converges to 0 since it can be estimated by some constant times $\left\|\chi_{2} u_{n}\right\|_{\mathscr{H}^{1}\left(\bar{U}_{2}\right)}$ by Lemma 2.4.9. Here, we have used that $\chi_{2} \uparrow_{U_{1}}=1$, i.e., the construction with the two sets $U_{1}$ and $U_{2}$ is necessary.

Therefore, the essential spectrum of a manifold describes the manifold at infinity, i.e., outside compact subsets, in contrast to the discrete spectrum determined by the behaviour of the metric on compact subsets. 


\section{Floquet theory}

The main idea in analysing periodic problems is the unitary equivalence of the operator on the full domain to a family of operators on a periodic cell, called Floquet theory or sometimes Bloch theory.

\subsection{Fourier analysis on abelian groups}

For the details of Fourier analysis on abelian groups see [Rud62] or [Pon57]. Let $\Gamma$ be a discrete, abelian group which is generated by $r$ elements $e_{1}, \ldots, e_{r} \in \Gamma$. Such a group is isomorphic to $\mathbb{Z}^{r_{0}} \times \mathbb{Z}_{p_{1}}^{r_{1}} \times \cdots \times \mathbb{Z}_{p_{a}}^{r_{a}}$ for some numbers $r_{0}, \ldots, r_{a} \in \mathbb{N}_{0}$ with $r=r_{0}+\cdots+r_{a}$. Here, $\mathbb{Z}_{p}$ denotes the abelian group of order $p \geq 2$ with one generator. We suppose that $\Gamma$ is endowed with its discrete topology.

We call the group of (continuous) homomorphism from $\Gamma$ into $\mathbb{S}^{1}$ the dual group or character group, denoted by $\hat{\Gamma}$. This group can be endowed in a natural way with a compact topology. The corresponding (unique) Haar measure with total mass 1 is denoted by $\mathrm{d} \theta$.

The dual group of $\mathbb{Z}$ is isomorphic (in the sense of topological groups) to $\mathbb{S}^{1}$. Furthermore, $\hat{\mathbb{Z}}_{p}$ is isomorphic to the closed subgroup $\left\{z \in \mathbb{C} \mid z^{p}=1\right\}$ (itself isomorphic to $\mathbb{Z}_{p}$ ) of $\mathbb{S}^{1}$. Therefore, we sometimes identify an element $\theta \in \hat{\Gamma}$ with its element $\left(\theta\left(e_{1}\right), \ldots, \theta\left(e_{r}\right)\right)$ in the corresponding subgroup of $\left(\mathbb{S}^{1}\right)^{r}$. In particular, if $\Gamma=\mathbb{Z}^{r}$ we sometimes identify the element $\theta \in \hat{\Gamma} \cong\left(\mathbb{S}^{1}\right)^{r}$ with $\left(\arg \left(\theta\left(e_{1}\right)\right), \ldots, \arg \left(\theta\left(e_{r}\right)\right)\right) \in \mathbb{R}^{r}$ (modulo $2 \pi$ in each component).

As in the case of the usual Fourier analysis on $\Gamma=\mathbb{Z}$, we have the following orthogonality relation

$$
\int_{\hat{\Gamma}} \theta(\gamma) \mathrm{d} \theta= \begin{cases}1 & \text { if } \gamma=1 \\ 0, & \text { otherwise }\end{cases}
$$

The Fourier inversion Formula in this context is

$$
f(\theta)=\sum_{\gamma \in \Gamma} \theta\left(\gamma^{-1}\right) \int_{\hat{\Gamma}} \theta^{\prime}(\gamma) f\left(\theta^{\prime}\right) \mathrm{d} \theta^{\prime}
$$

for allmost all $\theta \in \hat{\Gamma}$ and all $f \in L_{1}(\hat{\Gamma})$. Here, it is essential that $\Gamma$ is abelian.

In what follows we want the group $\Gamma$ to be of infinite order; thus the only restriction is $r_{0}>0$.

\subsection{Periodic manifolds and vector bundles}

For details see e.g. [Cha93, section 4.2] or [BGV92]. Let $\Gamma$ be a discrete, finitely generated group which acts isometrically on a $d$-dimensional Riemannian manifold $M^{\text {per }}$, i.e., we have a 
group action $\Gamma \times M^{\text {per }} \longrightarrow M^{\text {per }},(\gamma, x) \mapsto \gamma \cdot x$ such that $(\gamma \cdot): M^{\text {per }} \longrightarrow M^{\text {per }}$ is an isometry for every $\gamma \in \Gamma$.

We say that $\Gamma$ acts properly discontinuously on $M^{\text {per }}$ if to each $x \in M^{\text {per }}$ there is a neighborhood $U$ of $x$ such that the collection of open sets $\{\gamma U \mid \gamma \in \Gamma\}$ are pairwise disjoint (see Figure 3.1).

A manifold $M^{\text {per }}$ is called periodic or covering (Riemannian) manifold (with respect to $\Gamma$ ) if $\Gamma$ acts isometrically and properly discontinuously on $M^{\mathrm{per}}$.

Under these conditions, the orbit space $M^{\text {per }} / \Gamma$, i.e., the space of all equivalence classes $\Gamma x=\{\gamma \cdot x \mid \gamma \in \Gamma\}$, has a natural Riemannian metric such that the projection $\pi: M^{\text {per }} \longrightarrow$ $M^{\text {per }} / \Gamma, x \mapsto \Gamma x$ is a local isometry.

We call the action cocompact if the orbit space $M^{\text {per }} / \Gamma$ is compact.

3.2.1. Definition. An open set $D \subset M^{\text {per }}$ is called a fundamental domain of the $\Gamma$-action if $M:=\bar{D}$ is connected, if $\partial M$ is piecewise smooth and if the following two conditions are satisfied:

$$
\begin{gathered}
D \cap \gamma D=\emptyset \quad \text { for all } \gamma \in \Gamma \backslash\{1\} \\
\bigcup_{\gamma \in \Gamma} \gamma M=M^{\text {per }} .
\end{gathered}
$$

We call $M$ a period cell.

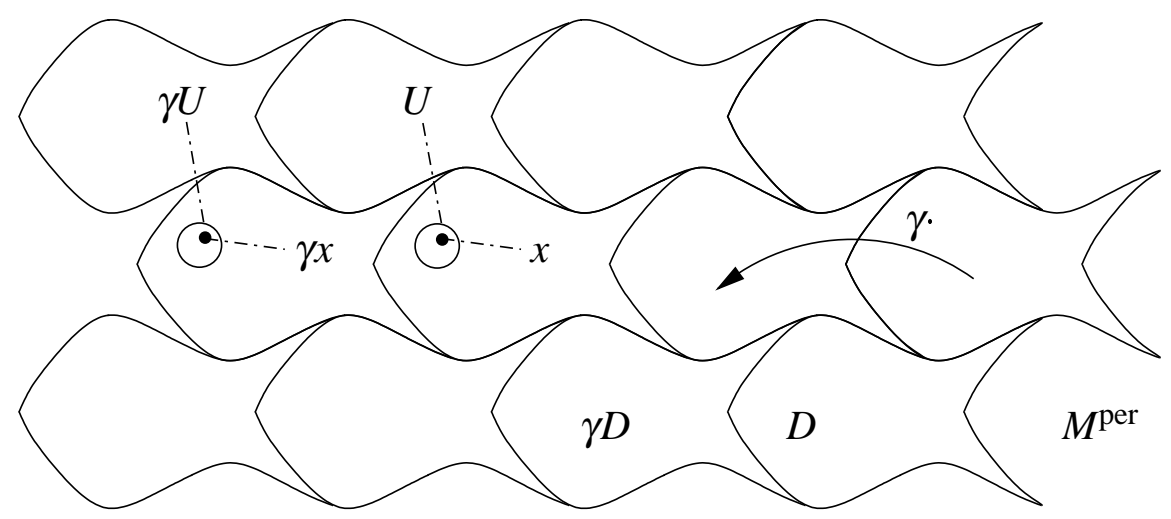

Figure 3.1: The group $\Gamma=\mathbb{Z}^{2}$ acting properly discontinuously on $M^{\text {per }}$. An element $\gamma \in \Gamma$ translates the fundamental domain $D$ to the disjoint copy $\gamma D$. We call the closure $M=\bar{D}$ a period cell.

Intuitively, if we fix a period cell $M$, one can imagine $M^{\text {per }}$ as a manifold glued from ( $\operatorname{card} \Gamma)$-many copies of $M$ such that $\gamma \in \Gamma$ moves $M$ to exactly one of the copies. Here, one can think of a tesselated pavement as in Figure 3.1 where we have chosen a fish-shaped period cell. Note that for a cocompact action any period cell is compact. For more details on fundamental domains, e.g. the existence of fundamental domains with piecewise smooth boundary see [Bea83, Chapter 9]. In particular, we have the following lemma:

3.2.2. Lemma. Suppose $\Gamma$ has $r$ generators. Then we can decompose the piecewise smooth boundary $\partial M$ into $2 r^{\prime}$ closed smooth components $Z^{i}$ with $r^{\prime} \geq r$ such that $Z^{i}$ are pairwise disjoint for $i=1, \ldots, 2 r^{\prime}$. Furthermore, for each $i=1, \ldots, r^{\prime}$ there exists $\gamma_{i} \in \Gamma$ such that 


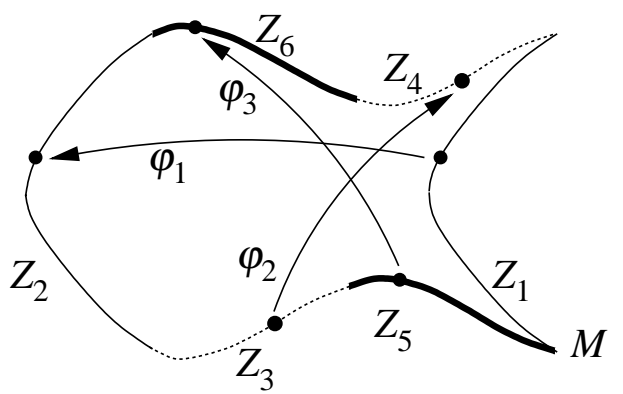

Figure 3.2: A period cell with the isometries $\varphi_{i}$ identifying elements on the boundary.

$\varphi_{i}: Z^{2 i-1} \longrightarrow Z^{2 i}$ are isometries of the form $\varphi_{i}(x)=\gamma_{i} \cdot x$ for all $x \in Z^{2 i-1}$ (see Figure 3.2). If we identify $Z^{2 i-1}$ with $Z^{2 i}$ via $\varphi_{i}$ we obtain a space isometric to the orbit space $M^{\text {per }} / \Gamma$.

Let $p: E^{\text {per }} \longrightarrow M^{\text {per }}$ be a hermitian vector bundle. We further suppose that $\Gamma$ acts on the left of both $E^{\text {per }}$ and $M^{\text {per }}$ in such a way that $p\left(\gamma_{*} e\right)=\gamma \cdot p(e)$ for all $e \in E^{\text {per }}$, i.e., we have maps

$$
\gamma_{*}: E_{x} \longrightarrow E_{\gamma \cdot x} \quad \text { for each } x \in M^{\text {per }}
$$

which we further suppose to be unitary with respect to $\langle\cdot, \cdot\rangle_{E_{x}}$. We call such a hermitian vector bundle periodic (with respect to $\Gamma$ ) or $\Gamma$-equivariant (see [BGV92, section 1.1]). If $E^{\text {per }}=\mathbf{A}^{p} T M^{\text {per }}$ is the exterior tangent bundle we define $\gamma_{*}$ to be the pull-back of

$$
\mathrm{d}\left(\gamma^{-1} \cdot\right): T_{\gamma \cdot x} M^{\text {per }} \longrightarrow T_{x} M^{\text {per }},
$$

the derivative of the isometry $\left(\gamma^{-1} \cdot\right)$.

For $u \in C_{c}^{\infty}\left(E^{\text {per }}\right)$ and $\gamma \in \Gamma$, we define the $\gamma$-translate of $u$ by

$$
\left(T_{\gamma} u\right)(y):=\gamma_{*} u\left(\gamma^{-1} \cdot y\right) \quad \text { for } y \in M^{\text {per }} .
$$

Since $\Gamma$ acts unitarily on $E^{\text {per }}$ and isometrically on $M^{\text {per }}$ we have

$$
\left\|T_{\gamma} u\right\|_{L_{2}\left(E^{\mathrm{per}}\right)}^{2}=\|u\|_{L_{2}\left(E^{\mathrm{per}}\right)}^{2} \quad \text { for } u \in C_{c}^{\infty}\left(E^{\mathrm{per}}\right) .
$$

Hence we can extend the operator $T_{\gamma}$ to a unitary operator on $L_{2}\left(E^{\text {per }}\right)$ also denoted by $T_{\gamma}$. We therefore have a unitary representation of $\Gamma$ on $L_{2}\left(E^{\text {per }}\right)$. The operator $T_{\gamma}$ acts as a translation operator.

\subsection{Floquet decomposition}

For details on Floquet theory on manifolds and vector bundles see [Don81] and [Gru98]. In the case $\Gamma=\mathbb{Z}^{d}$ and $M=\mathbb{R}^{d}$ we refer to [RS78].

Remember that $\Gamma$ is abelian. We start with the decomposition of the space $L_{2}\left(E^{\text {per }}\right)$. We define the space of smooth $(\Gamma, \theta)$-equivariant or $\theta$-periodic sections on $E^{\text {per }}$ by

$$
C^{\infty}\left(E^{\mathrm{per}}\right)^{\Gamma, \theta}:=\left\{u \in C^{\infty}\left(E^{\mathrm{per}}\right) \mid T_{\gamma} u=\theta(\gamma) u \text { for all } \gamma \in \Gamma\right\} .
$$


Clearly, any section $u \in C^{\infty}\left(E^{\text {per }}\right)^{\Gamma, \theta}$ is determined by its values $u \uparrow_{M}$ on a period cell $M \subset M^{\text {per }}$. Thus

$$
\|u\|_{L_{2}(E)}^{2}:=\int_{M}|u(x)|_{E_{x}}^{2} \mathrm{~d} x
$$

defines a norm on $C^{\infty}\left(E^{\text {per }}\right)^{\Gamma, \theta}$. We call the completion of $C^{\infty}\left(E^{\text {per }}\right)^{\Gamma, \theta}$ under this norm $L_{2}\left(E^{\text {per }}\right)^{\Gamma, \theta}$. If $E:=E^{\text {per }} \uparrow_{M}$ denotes the restricted vector bundle, it is easy to see that the restriction map $L_{2}\left(E^{\mathrm{per}}\right)^{\Gamma, \theta} \rightarrow L_{2}(E), u \mapsto u \uparrow_{M}$ is unitary. Furthermore,

$$
\left\|u \uparrow_{M}\right\|_{L_{2}\left(E^{\mathrm{per}}{ }_{M}\right)}=\left\|u \uparrow_{M^{\prime}}\right\|_{L_{2}\left(E^{\mathrm{per}} \Gamma_{M^{\prime}}\right)}
$$

for $\theta$-periodic $u \in L_{2}\left(E^{\mathrm{per}}\right)^{\Gamma, \theta}$ and any other periodic cell $M^{\prime}$. The inverse of the restriction map is given by extending a section $u \in L_{2}(E)$ on $M$ to a $\theta$-periodic section $\Theta^{\theta} u$ on $M^{\text {per }}$, i.e.,

$$
\left(\Theta^{\theta} u\right)(y):=\sum_{\gamma \in \Gamma} \theta\left(\gamma^{-1}\right) T_{\gamma} u(y) .
$$

Note that the $\theta$-periodicity is not a restriction in the case of $L_{2}$-spaces since we do not need any smoothness conditions at the boundary $\partial M$ for functions in $L_{2}$. Finally note that $\Theta^{\theta} u$ makes also sense for $u \in C_{c}^{\infty}\left(E^{\text {per }}\right)$.

In the sequel we often switch between a $\theta$-periodic section on $M^{\text {per }}$ and its restriction to a period cell $M$ (also called $\theta$-periodic). We set

$$
C_{\theta}^{\infty}(E):=\left\{u \uparrow_{M} \mid u \in C^{\infty}\left(E^{\mathrm{per}}\right)^{\Gamma, \theta}\right\} .
$$

Note that this space depends on $\theta$ in contrast to the corresponding $L_{2}$-space.

We define the direct (constant) fiber integral $\int_{\hat{\Gamma}}^{\oplus} L^{2}(E) \mathrm{d} \theta$ as in [RS78, XIII.16]. For a similar definition of nonconstant fiber integrals over $\theta$-associated vector bundles see e.g. [Don81] or [Gru98].

For $u \in C_{c}^{\infty}\left(E^{\text {per }}\right)$ we set $(U u)^{\theta}:=\Theta^{\theta} u$. Furthermore, we have

$$
\begin{aligned}
\|U u\|_{\int_{\hat{\Gamma}}^{\oplus} L_{2}(E) \mathrm{d} \theta}^{2} & =\int_{\hat{\Gamma}}\left\|(U u)^{\theta}\right\|_{L_{2}(E)}^{2} \mathrm{~d} \theta \\
& =\int_{\hat{\Gamma}} \int_{M} \sum_{\gamma, \gamma^{\prime} \in \Gamma} \theta\left(\gamma^{-1} \gamma^{\prime}\right)\left\langle T_{\gamma} u(y), T_{\gamma^{\prime}} u(y)\right\rangle_{E_{y}} \mathrm{~d} y \mathrm{~d} \theta \\
& =\sum_{\gamma \in \Gamma} \int_{M}\left|T_{\gamma} u(y)\right|_{E_{y}}^{2} \mathrm{~d} y \\
& =\sum_{\gamma \in \Gamma} \int_{\gamma M}|u(x)|_{E_{x}}^{2} \mathrm{~d} x \\
& =\int_{M^{\mathrm{per}}}|u(x)|_{E_{x}}^{2} \mathrm{~d} x \\
& =\|u\|_{L_{2}\left(M^{\mathrm{per}}\right)}^{2} .
\end{aligned}
$$

Thus this map extents to an isometry

$$
U: L_{2}\left(E^{\text {per }}\right) \longrightarrow \int_{\hat{\Gamma}}^{\oplus} L_{2}(E) \mathrm{d} \theta .
$$


The adjoint is given by

$$
U^{*} f(x)=\int_{\hat{\Gamma}} \theta(\gamma) \gamma_{*}^{-1} f^{\theta}(y) \mathrm{d} \theta \in E_{x}
$$

for $x \in M^{\text {per }}$ which can be written as $x=\gamma^{-1} \cdot y$ with $y \in M$ in a unique way (up to a set of measure 0). By the Fourier inversion Formula (3.2) we have

$$
\begin{aligned}
\left(U U^{*} f\right)^{\theta}(y) & =\sum_{\gamma \in \Gamma} \theta\left(\gamma^{-1}\right) T_{\gamma}\left(U^{*} f\right)(y) \\
& =\sum_{\gamma \in \Gamma} \theta\left(\gamma^{-1}\right) \gamma_{*} \int_{\hat{\Gamma}} \theta^{\prime}(\gamma) \gamma_{*}^{-1} f^{\theta^{\prime}}(y) \mathrm{d} \theta^{\prime} \\
& =\sum_{\gamma \in \Gamma} \theta\left(\gamma^{-1}\right) \int_{\hat{\Gamma}} \theta^{\prime}(\gamma) f^{\theta^{\prime}}(y) \mathrm{d} \theta^{\prime} \\
& =f^{\theta}(y) .
\end{aligned}
$$

We therefore have proven:

3.3.1. Theorem. The operator $U$ is unitary with adjoint $U^{*}$.

We now start with our analysis of periodic operators. For details see e.g. [RS78, section XIII.16].

3.3.2. Definition. A bounded operator $A$ in $L_{2}\left(E^{\text {per }}\right)$ is called periodic (with respect to $\Gamma$ ) if $A$ commutes with the unitary translation operator $T_{\gamma}$ for all $\gamma \in \Gamma$.

3.3.3. Definition. A bounded operator $\tilde{A}$ in $\int_{\hat{\Gamma}}^{\oplus} L_{2}(E) \mathrm{d} \theta$ is called decomposable if there exists a measurable family $\left(A^{\theta}\right)_{\theta}$ of bounded operators in $L_{2}(E)$ such that $(\tilde{A} f)^{\theta}=A^{\theta} f^{\theta}$ for every element $f \in \int_{\hat{\Gamma}}^{\oplus} L_{2}(E) \mathrm{d} \theta$. We write $\tilde{A}=\int_{\hat{\Gamma}}^{\oplus} A^{\theta} \mathrm{d} \theta$.

We have the following characterisation of decomposable operators:

3.3.4. Theorem. The decomposable bounded operators are exactly the operators commuting with each element of the algebra

$$
\mathscr{A}:=\left\{\int_{\hat{\Gamma}}^{\oplus} f(\theta) \mathbb{1} \mathrm{d} \theta \mid f \in L_{\infty}(\hat{\Gamma})\right\}
$$

consisting of those operators whose fibres are multiples of the identity operator $\mathbb{1}$ on $L_{2}(E)$.

Proof. A proof can be found in [RS78, Theorem XIII.84].

A consequence is:

3.3.5. Corollary. Let $A$ be a bounded operator in $L_{2}\left(E^{\text {per }}\right)$ and set $\widetilde{A}:=U A U^{*}$. Then $A$ is periodic if and only if $\widetilde{A}$ is decomposable. 


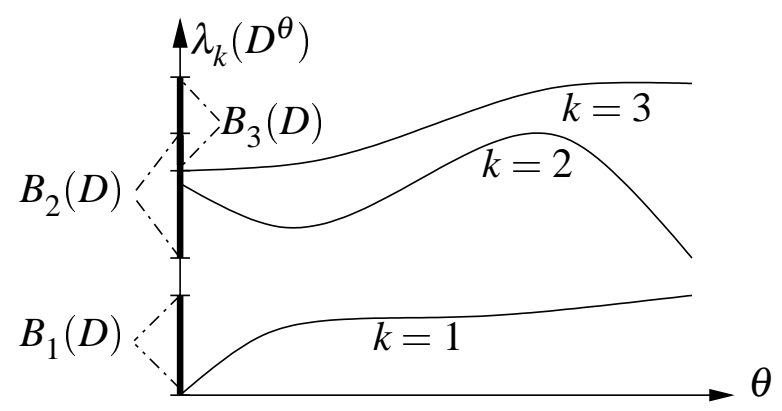

Figure 3.3: Band structure of the spectrum of a $\Gamma$-periodic operator $D$. Here, the second and third band overlap.

Proof. By Theorem 3.3.4, the set of all decomposable operators is the commutant $\mathscr{A}^{\prime}$ of $\mathscr{A}$. The subalgebra of $C(\hat{\Gamma})$, generated by $\{\tilde{\gamma} \mid \gamma \in \Gamma\}$, is dense in $C(\hat{\Gamma})$ by the Stone-Weierstrass Theorem. Here, we have set $\widetilde{\gamma}(\theta):=\theta(\gamma)$. Furthermore $C(\hat{\Gamma})$ is weakly dense in $L_{\infty}(\hat{\Gamma})$, therefore the subalgebra of $\mathscr{A}$ generated by $\widetilde{\gamma} \mathbb{1}$ is weakly dense in $\mathscr{A}$. Since commutants are weakly closed, an operator $\widetilde{A}$ lies in $\mathscr{A}^{\prime}$ if and only if $[\widetilde{A}, \widetilde{\gamma}]=0$ for all $\gamma \in \Gamma$. Finally, a calculation similar to (3.10) shows that

$$
U T_{\gamma} U^{*} f=\tilde{\gamma} f
$$

i.e., $\Gamma$ acts on $\int_{\hat{\Gamma}}^{\oplus} L_{2}(E) \mathrm{d} \theta$ as multiplication operator, i.e., $(\tilde{\gamma} f)^{\theta}=\theta(\gamma) f^{\theta}$. Therefore $[\widetilde{A}, \tilde{\gamma}]=$ 0 for all $\gamma \in \Gamma$ if and only if $\left[A, T_{\gamma}\right]=0$ for all $\gamma \in \Gamma$. The latter statement is the periodicity of A.

Since we are dealing with self-adjoint unbounded operators $D$ we apply our definitions to the (bounded) resolvent $(D+i)^{-1}$ (again see [RS78]).

The following nice characterisation of the spectrum of a decomposable operator is one reason why one is interested in Floquet theory:

3.3.6. Theorem. Suppose that $\left(D^{\theta}\right)_{\theta}$ is a family of positive operators on $L_{2}(E)$ such that $D^{\theta}$ has purely discrete spectrum $\lambda_{k}\left(D^{\theta}\right)$ written in increasing order and repeated according to multiplicity. Suppose further that $\lambda_{k}\left(D^{\theta}\right)$ depends continuously on $\theta$ for each $k \in \mathbb{N}$. Then the spectrum of $\int_{\hat{\Gamma}}^{\oplus} D^{\theta} \mathrm{d} \theta$ is given by

$$
\operatorname{spec} \int_{\hat{\Gamma}}^{\oplus} D^{\theta} \mathrm{d} \theta=\bigcup_{\theta \in \hat{\Gamma}} \operatorname{spec} D^{\theta}=\bigcup_{k \in \mathbb{N}}\left\{\lambda_{k}\left(D^{\theta}\right) \mid \theta \in \hat{\Gamma}\right\} .
$$

In particular, the spectrum of $\int_{\hat{\Gamma}}^{\oplus} D^{\theta} \mathrm{d} \theta$ consists of the union of compact intervals $B_{k}:=$ $\left\{\lambda_{k}\left(D^{\theta}\right) \mid \theta \in \hat{\Gamma}\right\}$, called $k$-th band.

Proof. For a proof see [RS78]. Since the eigenvalues depend continuously on $\theta$, and $\hat{\Gamma}$ is compact, the $k$-th band $B_{k}$ is indeed a compact interval.

Therefore the spectrum of a $\Gamma$-periodic operator $D$ with decomposition $\int_{\hat{\Gamma}}^{\oplus} D^{\theta} \mathrm{d} \theta$ (by Corollary 3.3.5) satisfying the assumptions of Theorem 3.3.6 has band structure, i.e., the spectrum 
is the locally finite union of compact intervals

$$
\operatorname{spec} D=\bigcup_{k \in \mathbb{N}} B_{k}(D)
$$

with $B_{k}(D)=\left\{\lambda_{k}\left(D^{\theta}\right) \mid \theta \in \hat{\Gamma}\right\}$, the $k$-th band of $D$. Note that in general we do not know if we have gaps or not, i.e., if $B_{k}(D) \cap B_{k+1}(D)=\emptyset$ for some $k \in \mathbb{N}$ (see Figure 3.3).

\subsection{Periodic Laplacian on a manifold}

Let $M^{\text {per }}$ be a periodic manifold as in Section 3.2. For simplicity, suppose that $M^{\text {per }}$ has no boundary. Denote by $\mathbf{A}^{p} T M$ the restriction of $\mathbf{A}^{p} T M^{\text {per }}$ to a period cell $M$ of $M^{\text {per }}$.

3.4.1. Definition. Let $\theta \in \hat{\Gamma}$. We denote by $\mathscr{H}_{\theta}^{1}\left(\mathbf{A}^{p} T M\right)$ the completion of $C_{\theta}^{\infty}\left(\mathbf{A}^{p} T M\right)$, the space of all $\theta$-periodic differential forms (see (3.9) and (3.5)), under the form norm of $q_{\mathbf{A}^{p} T M}^{\theta}$ or under the equivalent norm $\|\cdot\|_{\mathscr{H}^{1}\left(\mathbf{A}^{p} T M\right)}$. Here, $q_{\mathbf{A}^{p} T M}^{\theta}(u)$ is defined as in (2.13) for $u \in$ $C_{\theta}^{\infty}\left(\mathbf{A}^{p} T M\right)$ and can be extended to $\operatorname{dom} q_{\mathbf{A}^{p} T M}=\mathscr{H}_{\theta}^{1}\left(\mathbf{A}^{p} T M\right)$. Clearly, this defines a closed, positive form. We denote the corresponding operator by $\Delta_{\mathbf{A}^{p} T M}^{\theta}$ resp. $\Delta_{M}^{\theta}$ if $p=0$, the $\theta$ periodic Laplacian on $\mathbf{A}^{p}$ TM resp. $M$.

We have the following equivalent description of $\mathscr{H}_{\theta}^{1}(M)$ : We decompose the boundary $\partial M$ into $2 r^{\prime}$ components $Z^{i}$ as in Lemma 3.2.2 and obtain isometries $\varphi_{i}: Z^{2 i-1} \longrightarrow Z^{2 i}$. The restriction $u \uparrow_{\partial M}$ is well-defined for a $\theta$-periodic element $u \in \mathscr{H}_{\theta}^{1}(M)$ by Theorem 2.2.5. Therefore $u\left(\varphi_{i}(x)\right)=\theta\left(\gamma_{i}\right) u(x)$ for all $x \in Z^{2 i-1}$ and all $i=1, \ldots, r^{\prime}$, i.e., the value of $u$ on $Z^{2 i}$ is just the value of $u$ on $Z^{2 i-1}$ multiplied by a complex number of norm 1 .

After the completion procedure, $\mathscr{H}_{\theta}^{1}\left(\mathbf{A}^{p} T M\right)$ still depends on $\theta$ in contrast to the corresponding $L_{2}$-space (see Section 3.3). Note that if $u$ is smooth enough, similar remarks hold for higher derivatives like $\mathrm{d} u$.

3.4.2. Lemma. Let $M$ be a period cell of a periodic manifold $M^{\mathrm{per}}$. Suppose further that $\theta \in \hat{\Gamma}$. Then the spectrum of the $\theta$-periodic Laplacian $\Delta_{M}^{\theta}$ is purely discrete and satisfies

$$
\lambda_{k}^{\mathrm{N}}(M) \leq \lambda_{k}^{\theta}(M) \leq \lambda_{k}^{\mathrm{D}}(M) .
$$

This inequality is sometimes called Dirichlet-Neumann enclosure.

Furthermore, $\Delta_{\mathbf{A}^{p} T M}^{\theta}$ has purely discrete spectrum denoted by $\lambda_{k}^{\theta}\left(\mathbf{A}^{p} T M\right)$.

Proof. By Lemma 2.3.4, $\Delta_{M}^{\mathrm{N}}$ has purely discrete spectrum. We have the following inclusion

$$
C_{c}^{\infty}(M) \subset C_{\theta}^{\infty}(M) \subset C^{\infty}(M) .
$$

If we complete these spaces the inclusions remain true. Therefore we have

$$
\stackrel{\mathscr{H}}{ }^{1}(M) \subset \mathscr{H}_{\theta}^{1}(M) \subset \mathscr{H}^{1}(M) .
$$

In particular, the Min-max Principle (see Corollary 1.3.5) implies that $\Delta_{M}^{\theta}$ has purely discrete spectrum and the Inequality (3.12) is satisfied.

In the same way as in Lemma 2.3.4 we prove that $\Delta_{\mathbf{A}^{p} T M}^{\theta}$ has purely discrete spectrum denoted by $\lambda_{k}^{\theta}\left(\mathbf{A}^{p} T M\right)$. It is important that $\mathscr{H}_{\theta}^{1}\left(\mathbf{A}^{p} T M\right)$ and dom $q_{\mathbf{A}^{p} T M}^{\theta}$ have equivalent norms. This can be shown since the boundary term in the Gauss-Green Formula (2.17) vanishes for $\theta$-periodic forms (see e.g., [Hör85, Chapter 20.1] or [AC93]). 
Note that we still have an eigenvalue enclosure like (3.12) for the "Neumann" Laplacian on $\mathbf{A}^{p} T M$ (defined in the same way as for functions, i.e., the form domain is the completion of $C^{\infty}\left(\mathbf{A}^{p} T M\right)$ under the form norm of the quadratic form defined in (2.13)). But the "Neumann" Laplacian has infinite dimensional kernel hence it has no longer purely discrete spectrum. Thus we only obtain the trivial lower bound $\lambda_{k}^{\mathrm{N}}\left(\mathbf{A}^{p} T M\right)=0$ for all $k \in \mathbb{N}$. Here, the norms on $\mathscr{H}^{1}\left(\mathbf{A}^{p} T M\right)$ and $\operatorname{dom} q_{\mathbf{A}^{p} T M}^{\mathrm{N}}$ are no longer equivalent (see e.g., [AC93]).

Next, we note that the eigenvalues $\lambda_{k}\left(\mathbf{A}^{p} T M\right)$ of $\Delta_{\mathbf{A}^{p} T M}^{\theta}$ depend continuously on $\theta$ :

3.4.3. Theorem. Denote the eigenvalues of $\Delta_{\mathbf{A}^{p} T M}^{\theta}$ by $\lambda_{k}^{\theta}\left(\mathbf{A}^{p} T M\right)$. Then the function $\theta \mapsto$ $\lambda_{k}^{\theta}\left(\mathbf{A}^{p} T M\right)$ is continuous for each $k \in \mathbb{N}$.

Proof. (see Lemma 2.2 of [BJR99] or Theorem XIII.89 of [RS78] for the special case $M=\mathbb{R}^{d}$ and $\left.\Gamma=\mathbb{Z}^{d}\right)$

We apply Floquet theory to prove the following fundamental result:

3.4.4. Theorem. The spectrum of the Laplacian on $p$-forms on a periodic manifold $M^{\text {per }}$ has band structure, i.e.,

$$
\operatorname{spec} \Delta_{\mathbf{A}^{p} T M^{\mathrm{per}}}=\bigcup_{k \in \mathbb{N}} B_{k}\left(\Delta_{\mathbf{A}^{p} T M^{\mathrm{per}}}\right)
$$

with $B_{k}\left(\Delta_{\mathbf{A}^{p} T M^{\mathrm{per}}}\right)=\left\{\lambda_{k}^{\theta}\left(\mathbf{A}^{p} T M\right) \mid \theta \in \hat{\Gamma}\right\}$ being a compact interval, called the $k$-th band of $\Delta_{\mathbf{A}^{p} T M^{\mathrm{per}}}$.

Proof. Note that $\Delta_{\mathbf{A}^{p} T M^{\text {per }}}$ is $\Gamma$-periodic since the corresponding quadratic form $q_{\mathbf{A}^{p} T M^{\text {per }}}$ satisfies

$$
q_{\mathbf{A}^{p} T M^{\mathrm{per}}}\left(T_{\gamma} u\right)=\left\|\mathrm{d} T_{\gamma} u\right\|^{2}+\left\|\mathrm{d}^{*} T_{\gamma} u\right\|^{2}=q_{\mathbf{A}^{p} T M^{\mathrm{per}}}(u)
$$

for all $u \in \mathscr{H}^{1}\left(\mathbf{A}^{p} T M^{\text {per }}\right)$. Here, we have used the fact that the translation operators $T_{\gamma}$ commutes with the exterior derivative d, i.e.,

$$
\mathrm{d} T_{\gamma}=T_{\gamma} \mathrm{d}
$$

and that $T_{\gamma}$ acts unitarily on $L_{2}\left(\mathbf{A}^{p+1} T M^{\text {per }}\right)$ resp. $L_{2}\left(\mathbf{A}^{p-1} T M^{\text {per }}\right)$. The result follows from Floquet Theory (cf. Section 3.3).

We conclude some easy facts about the first band of $\Delta_{M^{\text {per }}}$.

3.4.5. Lemma. Suppose that $M^{\mathrm{per}}$ is connected. In the nonperiodic case $\theta \neq 1$ we have $\lambda_{k}^{\theta}(M)>0$ for all $k \in \mathbb{N}$. In particular, the first band of $\Delta_{M^{\mathrm{per}}}$ has non-empty interior.

Proof. Note that the eigenvalue 0 corresponds to functions constant on every component. If $M^{\text {per }}$ is connected we can choose a connected period cell $M$. Therefore the eigenfunction must be constant. But the constant lies only in the domain of the periodic Laplacian (quadratic form). The second statement follows from the preceding theorem.

We can apply the Hodge $*$-operator to obtain the following result: 
3.4.6. Theorem. The eigenvalues of $\Delta_{\mathbf{A}^{p} T M}^{\theta}$ and $\Delta_{\mathbf{A}^{d-p} T M}^{\theta}$ are the same, i.e., $\lambda_{k}^{\theta}\left(\mathbf{A}^{p} T M\right)=$ $\lambda_{k}^{\theta}\left(\mathbf{A}^{d-p} T M\right)$ for all $k \in \mathbb{N}$ and $0 \leq p \leq d$. In particular,

$$
\operatorname{spec} \Delta_{\mathbf{A}^{p} T M^{\text {per }}}=\operatorname{spec} \Delta_{\mathbf{A}^{p-d} T M^{\text {per }}} .
$$

Proof. The proof is essentially the same as the proof of Theorem 2.3.8. Note that $u$ is $\theta$ periodic if and only if $* u$ is $\theta$-periodic. The last statement follows by Theorem 3.4.4.

In the same way as in Theorem 2.3.9 we use supersymmetry to prove the following $\left(\mathbf{A}^{ \pm} T M\right.$ denotes the bundle of even resp. odd differential forms, see the notation in Theorem 2.3.9):

3.4.7. Theorem. We have $\operatorname{spec} \Delta_{\mathbf{A}^{+} T M}^{\theta} \backslash\{0\}=\operatorname{spec} \Delta_{\mathbf{A}^{-} T M}^{\theta} \backslash\{0\}$.

In dimension 2 the spectrum of the $\theta$-periodic Laplacian on forms is completely determined by the spectrum of the Laplacian on functions:

3.4.8. Corollary. Suppose that $M^{\mathrm{per}}$ is a connected 2-dimensional periodic manifold without boundary. Then

$$
\operatorname{spec} \Delta_{M^{\mathrm{per}}}=\operatorname{spec} \Delta_{\mathbf{A}^{1} T M^{\mathrm{per}}}=\operatorname{spec} \Delta_{\mathbf{A}^{2} T M^{\mathrm{per}}} \cdot
$$

Proof. This follows from the preceding two theorems and Theorem 3.4.4 except the fact that 0 lies in all spectra. The constant function is an eigenfunction of $\Delta_{M}^{\theta=1}$, i.e., $0 \in \operatorname{spec} \Delta_{M}^{\theta=1}$ and therefore $0 \in \operatorname{spec} \Delta_{\mathbf{A}^{2} T M}^{\theta=1}$ by Theorem 3.4.6. Furthermore, we have $0 \in \operatorname{spec} \Delta_{\mathbf{A}^{1} T M}^{\theta=1}$ since $\operatorname{ker} \Delta_{\mathbf{A}^{1} T M}^{\theta=1} \cong \operatorname{ker} \Delta_{\mathbf{A}^{1} T\left(M^{\mathrm{per}} / \Gamma\right)}$ is not trivial by the following arguments: The kernel of the Laplacian on 1-forms is isomorphic to the first cohomology group $H_{1}\left(M^{\mathrm{per}} / \Gamma\right)$ by de Rham's Theorem (cf. [Mas80, Appendix A]) and by the Hodge Theorem (cf. [Ros97, Theorem 1.45]). Since $M^{\text {per }}$ is connected and orientable by our global assumption, $M^{\text {per }} / \Gamma$ is a compact orientable surface and therefore homeomorphic to a sphere or to a connected sum of tori (see [Mas77, Theorem I.5.1]). Since the 2-sphere is simply connected the canonical projection $\pi: M^{\text {per }} \longrightarrow M^{\text {per }} / \Gamma$ which is also a covering map would have to be a homeomorphism (see [Mas77, Exercise V.6.1]. This is impossible since $M^{\text {per }}$ is non-compact. Therefore, $M^{\text {per }} / \Gamma$ is homeomorphic to a connected sum of $n$ tori and $\operatorname{dim} H_{1}\left(M^{\text {per }} / \Gamma\right)=2 n>0$ by [Mas80, Example III.4.2].

Theorem 3.4.6 allows us to prove the existence of gaps in the spectrum of $\Delta_{\mathbf{A}^{d} T M^{\text {per }}}$ provided $\Delta_{M^{\mathrm{per}}}$ has gaps in its spectrum. Furthermore, if $d=2$ then a gap in the spectrum of $\Delta_{M^{\mathrm{per}}}$ is automatically a gap in the spectrum of $\Delta_{\mathbf{A}^{p} T M^{\mathrm{per}}}$ for $p=1$ and $p=2$ by the preceding corollary. Therefore, our results on spectral gaps of the Laplacian on functions in Chapters 4, 5, 6 and 7 remain true in the case of $d$-forms resp. 1- and 2-forms if $d=2$.

\subsection{Harmonic extension}

We need some facts about harmonic functions on a manifold and the $\theta$-periodic harmonic extension of a function given on $X \subset M$ onto a connected period cell $M$.

First, we note that the boundary term of the Gauss-Green Formula vanishes for $\theta$-periodic functions. 
3.5.1. Lemma. Let $u \in \mathscr{H}_{\theta}^{2}(M)$ and $v \in \mathscr{H}_{\theta}^{1}(M)$. Then $\int_{\partial M} \partial_{\mathrm{n}} u \bar{v}=0$.

Proof. As in Lemma 3.2.2 we split $\partial M$ into $2 r^{\prime}$ smooth components $Z^{i}$ with the isometries $\varphi_{i}: Z^{2 i-1} \longrightarrow Z^{2 i}$ such that $\varphi_{i}(x)=\gamma_{i} \cdot x$. The function $f=\partial_{\mathrm{n}} u \bar{v}$ is well defined on $\partial M$ by Theorem 2.2.5. Furthermore, $\left(f \circ \varphi_{i}\right)(x)=f\left(\gamma_{i} \cdot x\right)=-\theta\left(\gamma_{i}\right) \overline{\theta\left(\gamma_{i}\right)} f(x)=-f(x)$ for $x \in Z^{2 i-1}$ since the normal derivatives on $Z^{2 i-1}$ and $Z^{2 i}$ have opposite sign. Note that $Z^{2 i-1}$ and $Z^{2 i}$ have opposite orientation. But $\varphi_{i}$ changes the orientation (see Figure 3.2), so we have

$$
\int_{\partial M} f=\sum_{i=1}^{r^{\prime}} \int_{Z^{2 i-1}}\left(f-f \circ \varphi_{i}\right)=0
$$

since $\int_{Z^{2 i}} f=-\int_{Z^{2 i-1}} f \circ \varphi_{i}$.

3.5.2. Lemma. Let $M^{\text {per }}$ be a periodic manifold with period cell $M$. Suppose that $X \subset M$ is closed and such that $M \backslash X$ has piecewise smooth boundary. Suppose further that $\gamma X \cap X \neq \emptyset$ implies $\gamma=1$. Let $h \in \mathscr{H}_{\theta}^{1}(M)$ be $\theta$-periodic. Then the following statements are equivalent:

1. $\langle\mathrm{d} h, \mathrm{~d} v\rangle_{L_{2}\left(T^{*} M\right)}=0$ for all $v \in C_{\theta}^{\infty}(M)$ with $\left.v\right|_{X}=0$.

2. The function $h$ is smooth and harmonic on $M \backslash X$, i.e., $\Delta h \uparrow_{M \backslash X}=0$ and $\mathrm{d} h$ is $\theta$-periodic on $M \backslash X$.

Proof. $(1) \Rightarrow(2)$ : By definition of $\mathscr{H}_{\theta}^{1}(M)$ as the closure of $C_{\theta}^{\infty}(M) \cong C^{\infty}\left(M^{\text {per }}\right)^{\Gamma, \theta}$ (see (3.5)) we can assume that $h$ extends $\theta$-periodically onto $M^{\text {per }}$ to an $\mathscr{H}^{1}$-function. Then we have

$$
0=\langle\mathrm{d} h, \mathrm{~d} v\rangle_{L_{2}\left(T^{*} M^{\prime}\right)}=\int_{M^{\prime} \backslash X}\langle\mathrm{~d} h, \mathrm{~d} v\rangle_{T^{*} M^{\prime}}=\int_{M^{\prime} \backslash X} h \overline{\Delta v}
$$

for all $v \in C_{c}^{\infty}\left(M^{\prime} \backslash X\right)$ and all period cells $M^{\prime}$. Note that the assumption is only made on $M$ but it remains true for any period cell $M^{\prime}$ via (3.7). The Weyl lemma yields $h \in C^{\infty}\left(\stackrel{\circ}{ }^{\prime} \backslash X\right)$ and $\Delta h=0$. By choosing different period cells $M^{\prime}$ such that $M^{\prime}$ covers some parts of $\partial M$ we can show that $h$ is smooth up to the boundary $\partial M$, i.e., $h \in C^{\infty}(M \backslash X)$.

$(2) \Rightarrow(1)$ : Without loss of generality we may assume that $X \subset \stackrel{M}{\text {. Then }} \partial(M \backslash X)=$ $\partial M \cup \dot{\partial} X$. The Gauss-Green Formula yields

$$
\int_{\partial(M \backslash X)} \partial_{\mathrm{n}} h \bar{v}=\int_{\partial M} \partial_{\mathrm{n}} h \bar{v}
$$

for all $v \in C_{\theta}^{\infty}(M)$ such that $v \Upsilon_{X}=0$. The latter integral vanishes since $\mathrm{d} h$ and $v$ are $\theta$-periodic (cf. Lemma 3.5.1).

3.5.3. Theorem. Suppose that the closed set $X \subset M$ has piecewise smooth boundary. Suppose further that $\gamma X \cap X \neq \emptyset$ implies $\gamma=1$. Then every $u \in \mathscr{H}^{1}(X)$ can be extended harmonically in a unique way to an element $h=\Phi u \in \mathscr{H}_{\theta}^{1}(M)$, i.e., $h$ is harmonic on $M \backslash X$ with $\theta$-periodic derivative $\mathrm{d} h$ on $M \backslash X$.

Proof. By the assumptions on $X$ there exists an open set $U$ containing $X$ such that $U$ has strictly positive distance from $\partial M$ (if necessary we have to choose another period cell $M$ which is possible by the assumption on $X$ ). 
Suppose first that we are in the nonperiodic case, i.e., $\theta \neq 1$. Then 0 is not an eigenvalue for $\Delta_{M}^{\theta}$. Therefore we can use $q_{M}^{\theta}$ as norm on $\widetilde{\mathscr{H}}:=\mathscr{H}_{\theta}^{1}(M)$ (see Section 1.5). For $u \in \mathscr{H}^{1}(X)$ let $v^{\prime} \in \mathscr{H}^{1}\left(M^{\text {per }}\right)$ be an arbitrary extension of $u$ with $\operatorname{supp} v^{\prime} \subset U$ (such extensions exist, see [Ste70]). Set $v:=\Theta^{\theta} v^{\prime}$ (see (3.8)). Then

$$
v(x)=\sum_{\gamma \in \Gamma} \theta(\gamma) v^{\prime}(\gamma \cdot x)=u(x)
$$

for all $x \in X$ since this sum consists only of the term for $\gamma=1$. Let $w:=P v$ be the orthogonal projection of $v$ onto the closed subspace

$$
\stackrel{\mathscr{H}}{\theta}_{\theta}^{1}(M \backslash X):=\left\{f \in \mathscr{H}_{\theta}^{1}(M) \mid f \uparrow_{X}=0\right\}
$$

of the Hilbert space $\widetilde{\mathscr{H}}$. We set $h:=v-w$. Then we have $h \uparrow_{X}=v \uparrow_{X}=u \uparrow_{X}$ and $h=v-P v \in$ $\left(\mathscr{H}_{\theta}^{1}(M \backslash X)\right)^{\perp}=\widetilde{\mathscr{H}}_{0}$. Note that elements of $\widetilde{\mathscr{H}}_{0}$ are harmonic on $M \backslash X$ and have $\theta$-periodic derivatives on $M \backslash X$ by Lemma 3.5.2. This proves the existence of a harmonic extension.

For the uniqueness we show that $0 \in \mathscr{H}^{1}(X)$ can only be extended to $0 \in \widetilde{\mathscr{H}}_{0}$ : Let $h$ be an extension of 0 . Then we have $h \in \dot{\mathscr{H}}_{\theta}^{1}(M \backslash X)$ because $h \uparrow_{X}=0$ and $h$ is $\theta$-periodic. By construction we have $h \in \widetilde{\mathscr{H}_{0}}=\left(\stackrel{\mathscr{H}}{\theta}_{\theta}^{1}(M \backslash X)\right)^{\perp}$ thus $h=0$.

In the periodic case we have to exclude the constant function $\mathbb{1}$ because its $\widetilde{\mathscr{H}}$-norm would be 0 . Therefore we set $\widetilde{\mathscr{H}}:=\mathscr{H}_{\theta=1}^{1}(M) / \mathbb{C} \mathbb{1}$. We denote the elements of this quotient space by $\widetilde{u}=u+\mathbb{C} \mathbb{1}$. As above let $v$ be a $\theta$-periodic extension of $u \in \mathscr{H}^{1}(X)$. Let $P$ be the orthogonal projection onto $\stackrel{\mathscr{H}}{\theta=1}_{\theta=1}^{1}(M \backslash X) / \mathbb{C} \mathbb{1}=\left\{\widetilde{u} \mid u \in \stackrel{\mathscr{H}}{\theta=1}_{\theta=1}(M \backslash X)\right\}$. Set $\widetilde{h}=\widetilde{v}-P \widetilde{v}$. Let $h$ be the representative of $\tilde{h}$ such that $h \uparrow_{X}=u \uparrow_{X}$. Again, by Lemma 3.5.2, $h$ is harmonic on $M \backslash X$ with periodic derivative $\mathrm{d} h$ on $M \backslash X$. The uniqueness follows from the choice of the representative $h$.

\subsection{Periodic coverings}

In this section, we prove that a $C^{k}$-bounded atlas $\mathscr{A}$ with finite maximal number of neighbours and a $C^{k}$-bounded partition of unity with strictly positive distance from the boundary exist. Furthermore, we construct uniformly elliptic and $C^{k-1}$-bounded metrics with respect to the atlas $\mathscr{A}$.

First, we construct a cover of $M^{\text {per }}$ adapted to the periodic structure. Fix a period cell $M$ of $M^{\text {per }}$ and choose a cover of $M$, i.e., finitely many sets $U_{\alpha}$ open in $M^{\text {per }}$ such that $M \subset \bigcup_{\alpha \in A} U_{\alpha}$. This is possible since $M$ is compact. Suppose that $\gamma U_{\alpha} \cap U_{\alpha} \neq \emptyset$ implies $\gamma=1$, i.e., $U_{\alpha}$ is smaller than some period cell (not necessarily $M$ ). Suppose further that $\varphi_{\alpha}: U_{\alpha} \longrightarrow V_{\alpha} \subset \mathbb{R}^{d}$ are charts. Without loss of generality we can assume that the transition maps $\varphi_{\alpha_{2}} \circ \varphi_{\alpha_{1}}^{-1}$ are bounded in $C^{k}$, i.e., all components and derivatives up to the order $k$ are supposed to be bounded functions, provided $U_{\alpha_{1}} \cap U_{\alpha_{2}} \neq \emptyset$. Then

$$
\varphi_{\alpha, \gamma}: U_{\alpha, \gamma} \longrightarrow V_{\alpha}
$$

with

$$
U_{\alpha, \gamma}:=\gamma U_{\alpha} \quad \text { and } \quad \varphi_{\alpha, \gamma}(\gamma \cdot x):=\varphi_{\alpha}(x)
$$


are also charts and $\left(U_{\alpha, \gamma}\right)_{\alpha \in A, \gamma \in \Gamma}$ form a covering of $M^{\text {per }}$. Coverings of $M^{\text {per }}$ arising in this way are called periodic covers.

Note that we have $\varphi_{\alpha_{2}, \gamma_{2}} \circ \varphi_{\alpha_{1}, \gamma_{1}}^{-1}=\varphi_{\alpha_{2}} \circ \varphi_{\alpha_{1}}^{-1}$ for all $\gamma_{1}, \gamma_{2} \in \Gamma^{\prime}$. Therefore the $C^{k}$-bound of the transition maps for the atlas $\mathscr{A}^{\text {per }}=\left(\varphi_{\alpha, \gamma}: U_{\alpha, \gamma} \longrightarrow V_{\alpha, \gamma}\right)_{\alpha}$ is the same as the $C^{k}$-bound of the transition maps for the atlas $\mathscr{A}=\left(\varphi_{\alpha}: U_{\alpha} \longrightarrow V_{\alpha}\right)_{\alpha}$ of the period cell $M$, i.e., on $M^{\text {per }}$ there exist $C^{k}$-bounded atlases (see Definition 2.2.1). This is one of the reasons why we choose a periodic cover; periodic manifolds can be treated essentially like compact manifolds.

To shorten the notation we write $\beta=(\alpha, \gamma) \in A \times \Gamma$.

3.6.1. Lemma. The number of neighbours of a given set $U_{\beta}$ is bounded, i.e., there exists a number $N \in \mathbb{N}$ such that

$$
\operatorname{card}\left\{\beta^{\prime} \in A \times \Gamma \mid U_{\beta} \cap U_{\beta^{\prime}} \neq \emptyset\right\} \leq N
$$

for all $\beta \in A \times \Gamma$.

Proof. Let $r$ denotes the number of generators $e_{1}, \ldots, e_{r}$ of $\Gamma$. For each $e_{i}$ we have two neighbours $e_{i} M$ and $e_{i}^{-1} M$ of $M$. Therefore $M$ has $3^{r}-1$ neighbours. Occasionally, a chart set $U_{\beta}$ could maximally have $N=3^{r}(\operatorname{card} A)$ neighbours.

Next, we construct a partition of unity adapted to the periodic structure.

3.6.2. Lemma. There exists a partition of unity $\left(\chi_{\alpha, \gamma}\right)$ subordinate to the covering $\left(U_{\alpha, \gamma}\right)$ such that $\chi_{\alpha, \gamma}=T_{\gamma} \chi_{\alpha, 1}$ for all $\alpha \in A, \gamma \in \Gamma$. Such a partition of unity is called periodic. In particular, the partition of unity is $C^{k}$ bounded and can be chosen such that a strictly positive distance from the boundary exists (see Definition 2.2.1).

Proof. Let $\pi: M^{\text {per }} \longrightarrow M^{\text {per }} / \Gamma$ be the projection onto the orbit space. Then $\widetilde{U}_{\alpha}:=\pi U_{\alpha}$ form an open covering of $M^{\text {per }} / \Gamma$. Suppose $\left(\widetilde{\chi}_{\alpha}\right)$ is a partition of unity subordinate to the atlas $\widetilde{\mathscr{A}}=\left(\widetilde{\varphi}_{\alpha}: \widetilde{U}_{\alpha} \longrightarrow V_{\alpha}\right)_{\alpha \in A}$. Without loss of generality we can assume that $\operatorname{dist}\left(\operatorname{supp} \tilde{\chi}, V_{\alpha} \backslash\right.$ $\left.\partial \mathbb{R}_{+}^{d}\right) \geq d_{0}$ for all $\alpha \in A$ and some $d_{0}>0$ since $A$ is finite.

We can lift any smooth function on the quotient space to the full space, i.e., we set $\chi_{\alpha}(x):=$ $\tilde{\chi}_{\alpha}(\pi x)$. Since $\gamma U_{\alpha} \cap U_{\alpha} \neq \emptyset$ implies $\gamma=1$, the function $\chi_{\alpha, \gamma}:=\chi_{\alpha} \uparrow_{U_{\alpha, \gamma}}$ is smooth and has compact support in $U_{\alpha, \gamma}$. Furthermore, if $x \in M^{\text {per }}$ then there exist $\alpha \in A$ and $\gamma \in \Gamma^{\prime}$ such that $x \in U_{\alpha, \gamma}$ and $\left(\sum_{\alpha, \gamma} \chi_{\alpha, \gamma}\right)(x)=\sum_{\alpha} \chi_{\alpha}(x)=\sum_{\alpha} \widetilde{\chi}_{\alpha}(\pi x)$. The boundedness of $\sup _{\alpha, \gamma}\left\|\chi_{\alpha, \gamma}\right\|_{C^{k}\left(V_{\alpha}\right)}$ and the strictly positive distance from the boundary follow from the periodicity of $\chi_{\alpha}$.

Finally, we state the following result:

3.6.3. Lemma. The periodic metric $\mathrm{g}^{\mathrm{per}}$ is uniformly elliptic with respect to $\mathscr{A}^{\mathrm{per}}$. Furthermore, $g^{\text {per }}$ is $C^{k}$-bounded if $\mathscr{A}^{\text {per }}$ is $C^{k+1}$-bounded.

Proof. Cleary, by the periodicity of $g^{\text {per }}$ and $\mathscr{A}^{\text {per }}$, we can pass to the compact quotient Riemannian manifold $M^{\text {per }} / \Gamma$. Here, every metric is uniformly elliptic, provided the metric components can be extended continuously onto the closure of $\widetilde{U}_{\alpha}$. The $C^{k+1}$-boundedness of $g^{\text {per }}$ follows in the same way. 


\section{Construction of a periodic manifold}

In this chapter we present a class of examples of periodic manifolds with gaps in the spectrum of the corresponding Laplacian. In the simplest case the periodic manifold is obtained by glueing together $\mathbb{Z}$ copies of a fixed compact manifold $X$ modified in the neighbourhood of two distinct points such that we have two small cylindrical ends. We obtain gaps in the spectrum if we shrink the radius of the cylindrical ends.

\subsection{Metric estimates}

First, we collect some facts about Riemannian manifolds. For details see e.g. [BGM71], [Cha93], [dC92] or [GHL87].

Let $X$ be a compact Riemannian manifold of dimension $d \geq 2$ (possibly with boundary $\partial X \neq \emptyset)$ and metric $g$. Let $B_{X}(x, \varepsilon)$ be the open geodesic ball at $x$ with radius $\varepsilon>0$. The injectivity radius $\operatorname{inj}_{X} X$ in $x$ is the maximal radius such that the exponential map is defined on $B_{X}(x, \varepsilon)$. The (global) injectivity radius inj $X$ is the infimum of $\operatorname{inj}_{x} X$ taken over all $x \in X$. On a compact manifold we always have $\varepsilon_{0}:=\operatorname{inj} X>0$.

The exponential map defines a chart map

$$
\exp _{x_{0}}: B_{\varepsilon_{0}} \longrightarrow B_{X}\left(x, \varepsilon_{0}\right) \subset X
$$

(called normal coordinates at $x_{0}$ ) where $B_{\varepsilon_{0}}$ denotes the centered open ball of radius $\varepsilon_{0}$ in the tangent space $T_{x_{0}} X$ at $x_{0}$. We identify this vector space with $\mathbb{R}^{d}$ by fixing the basis $\partial_{1}, \ldots, \partial_{d}$ of $T_{x_{0}} X$. If we choose polar coordinates $v=s \sigma \in \mathbb{R}^{d}$ with $0<s<\varepsilon_{0}$ and $\sigma \in \mathbb{S}^{d-1}=: \mathbb{S}$ to describe points $B_{\varepsilon_{0}} \backslash\{0\}$ we obtain a diffeomorphism

$$
\varphi:] 0, \varepsilon_{0}\left[\times \mathbb{S} \longrightarrow B_{X}\left(x, \varepsilon_{0}\right) \backslash\left\{x_{0}\right\} \subset X .\right.
$$

The Gauss lemma implies that the metric in polar coordinates (the induced metric on $] 0, \varepsilon_{0}[\times \mathbb{S}$ by $\varphi$ ) splits into a radial part and a spherical part, i.e.,

$$
\left(\varphi^{*} g\right)_{(s, \sigma)}=\mathrm{d} s_{s}^{2}+h_{(s, \sigma)}
$$

(see e.g. [GHL87, Lemma 2.93]) where $h_{(s, \cdot)}$ denotes a parameter-dependent metric on the $(d-1)$-dimensional unit sphere $\mathbb{S}=\mathbb{S}^{d-1}$.

Our glueing procedure requires that we flatten the manifold in the neighbourhood of some points. We need the following estimate: 
4.1.1. Lemma. Suppose the metric is given in polar coordinates at $x_{0}$ by (4.1). Denote the standard metric on $\mathbb{S}$ by $\mathrm{d} \sigma^{2}$. Then there exist constants $c>1$ and $0<s_{0}<\operatorname{inj} X$ such that

$$
\frac{1}{c^{2}} s^{2} \mathrm{~d} \sigma^{2} \leq h_{(s, \cdot)} \leq c^{2} s^{2} \mathrm{~d} \sigma^{2}
$$

in the sense that

$$
\frac{1}{c^{2}} s^{2} \mathrm{~d} \sigma_{\sigma}^{2}\left(u_{\sigma}, u_{\sigma}\right) \leq h_{(s, \sigma)}\left(u_{\sigma}, u_{\sigma}\right) \leq c^{2} s^{2} \mathrm{~d} \sigma_{\sigma}^{2}\left(u_{\sigma}, u_{\sigma}\right)
$$

for all $u_{\sigma} \in T_{\sigma} \mathbb{S}, \sigma \in \mathbb{S}$ and $0<s<s_{0}$.

Proof. Denote by $g_{i j}(v):=g_{\exp _{x_{0}} v}\left(\partial_{i}, \partial_{j}\right)$ the metric components in normal coordinates. Let $\lambda_{\min }(v)$ resp. $\lambda_{\max }(v)$ be the smallest resp. greatest eigenvalue of the corresponding matrix. Since this matrix is strictly positive we have $0<\lambda_{\min }(v)$. Furthermore the eigenvalues depend continuously on $v$ (since $\left(g_{i j}(v)\right)$ does, see e.g., [Kat66, Theorem II.5.1] or Lemma 1.4.5). Thus if we restrict ourselves to the smaller chart given by $|v| \leq s_{0}<\operatorname{inj} X$ for some $0<s_{0}<\operatorname{inj} X$ we can choose $c>0$ such that

$$
\frac{1}{c^{2}} \leq \lambda_{\min }(v) \leq \lambda_{\max }(v) \leq c^{2}
$$

We conclude

$$
\frac{1}{c^{2}}|u|^{2} \leq \sum_{i, j=1}^{d} g_{i j} u^{i} u^{j} \leq c^{2}|u|^{2}
$$

for any $u \in \mathbb{R}^{d}$. In polar coordinates the standard metric at $v=s \sigma$ is

$$
|u|^{2}=\mathrm{d} s_{s}^{2}\left(u_{s}, u_{s}\right)+s^{2} \mathrm{~d} \sigma_{\sigma}^{2}\left(u_{\sigma}, u_{\sigma}\right)
$$

with $u=u_{s}+u_{\sigma}$, where $u_{s}$ is the vector tangent to the radial direction and where $u_{\sigma}$ is the vector tangent to the sphere $\mathbb{S}$ at $v=s \sigma$. From (4.1) and (4.3) we conclude

$$
\frac{1}{c^{2}}\left(\mathrm{~d} s^{2}+s^{2} \mathrm{~d} \sigma^{2}\right) \leq\left(\varphi^{*} g\right)_{(s, \sigma)}=\mathrm{d} s^{2}+h_{(s, \sigma)} \leq c^{2}\left(\mathrm{~d} s^{2}+s^{2} \mathrm{~d} \sigma^{2}\right) .
$$

Therefore we have proven (4.2).

We need another type of estimates on the metric:

4.1.2. Lemma. Suppose we are given two positive bilinear forms $h_{1}$ and $h_{2}$ on a finite dimensional Hilbert space $V$ with orthonormal basis $e_{1}, \ldots, e_{d}$ which satisfy $h_{1} \leq h_{2}$, i.e., $h_{1}(u, u) \leq h_{2}(u, u)$ for all $u \in V$.

1. Denote by det $h_{k}$ the determinant of the matrix $\left(h_{k}\left(e_{i}, e_{j}\right)\right)_{i j}$. Then we have the inequality $\operatorname{det} h_{1} \leq \operatorname{det} h_{2}$.

2. Suppose $\alpha_{1}, \alpha_{2} \geq 0$ with $\alpha_{1}+\alpha_{2}=1$. Then the convex combination lies between the given forms, $i$. e. $h_{1} \leq \alpha_{1} h_{1}+\alpha_{2} h_{2} \leq h_{2}$. In particular, if $h_{1}$ is strictly positive so is the convex combination.

Proof. The first statement is a simple consequence of the Min-max Principle (Corollary 1.3.5): From the assumption we get $0 \leq \lambda_{k}\left(h_{1}\right) \leq \lambda_{k}\left(h_{2}\right)$ and thus $\operatorname{det} h_{1}=\lambda_{1}\left(h_{1}\right) \cdot \ldots \cdot \lambda_{d}\left(h_{1}\right) \leq$ $\lambda_{1}\left(h_{2}\right) \cdot \ldots \cdot \lambda_{d}\left(h_{2}\right)=\operatorname{det} h_{2}$.

The second statement is true in any ordered vector space. 

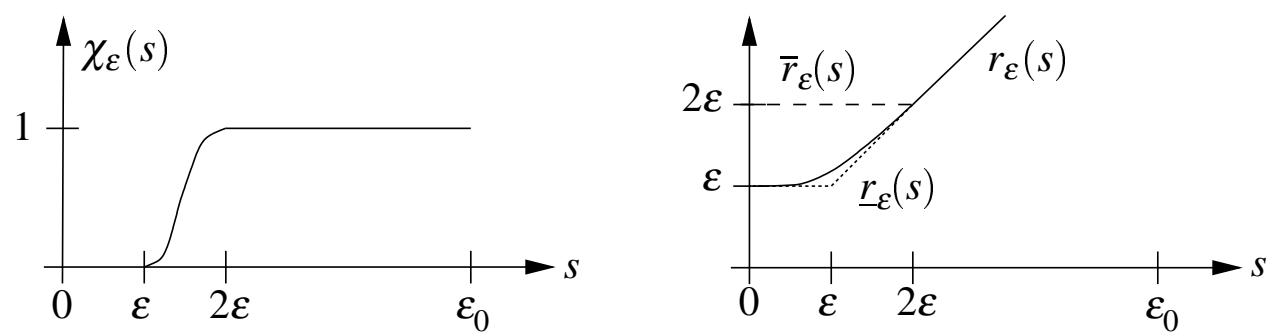

Figure 4.1: Graphs of the cut-off function $\chi_{\varepsilon}$ and of the radii functions $\underline{r}_{\varepsilon}, r_{\varepsilon}, \bar{r}_{\varepsilon}$.

\subsection{Construction of the period cell}

As above, let $X$ be a compact Riemannian manifold of dimension $d \geq 2$ (possibly with boundary $\partial X \neq \emptyset$ ). Let $r$ be the number of generators of a given abelian group $\Gamma$. We choose $2 r$ distinct points $x_{1}, \ldots, x_{2 r}$. For each point $x_{i}$ we set $B_{\varepsilon}^{i}:=B\left(x_{i}, \varepsilon\right)$. Suppose that the radius $\varepsilon_{0}>0$ of the geodesic ball around $x_{i}$ is smaller than the injectivity radius $\operatorname{inj} X$. Suppose further that $B_{\varepsilon_{0}}^{i}$ are pairwise disjoint and that $B_{\varepsilon_{0}}^{i}$ does not intersect the boundary of $X$ for all $i=1, \ldots, 2 r$. Denote by $B_{\varepsilon}$ the union of all these balls. Let $X_{\varepsilon}:=X \backslash B_{2 \varepsilon}$ for $0<2 \varepsilon<\varepsilon_{0}$ with metric inherited from $X$. Finally, let $Y_{\varepsilon}^{i}:=\partial B_{2 \varepsilon}^{i}$ be the component of the boundary of $X_{\varepsilon}$ near $x_{i}$ (see Figure 4.2).

We start now with the construction of the modified metric on $X$. Let $\chi_{\varepsilon}$ be a smooth function with values between 0 and $1, \chi_{\varepsilon}(s)=0$ for $s \leq \varepsilon$ and $\chi_{\varepsilon}(s)=1$ for $s \geq 2 \varepsilon$. Let $r_{\varepsilon}$ be a smooth function with $r_{\varepsilon}(s)=\varepsilon$ in a neighbourhood of 0 and $r_{\varepsilon}(s)=s$ for $s \geq 2 \varepsilon$. Finally, let $\underline{r}_{\varepsilon}(s)=\varepsilon$ for $s \leq \varepsilon$ resp. $\underline{r}_{\varepsilon}(s)=s$ for $s \geq \varepsilon$ and $\bar{r}_{\varepsilon}:=\underline{r}_{2 \varepsilon}$. Suppose $\underline{r}_{\varepsilon}$ and $\bar{r}_{\varepsilon}$ enclose $r_{\varepsilon}$ (see Figure 4.1).

From the orthogonal decomposition in polar coordinates (4.1) we obtain a metric $h_{(s, \cdot)}^{i}$ on the sphere $\mathbb{S}$ for each point $x_{i}$. We replace this metric by a convex combination of the standard metric on the sphere with radius $r_{\varepsilon}(s)$ and the original metric $h_{(s, \cdot)}^{i}$, i.e., we obtain a metric on $\mathbb{S}$ by

$$
h_{(\varepsilon, s, \sigma)}^{i}:=\left(1-\chi_{\varepsilon}(s)\right) r_{\varepsilon}^{2}(s) \mathrm{d} \sigma_{\sigma}^{2}+\chi_{\varepsilon}(s) h_{(s, \sigma)}^{i},
$$

depending on the two parameters $s$ and $\varepsilon$. By Lemma 4.1.2, $h_{(\varepsilon, s, \cdot)}^{i}$ is indeed strictly positive, thus a metric. Lemma 4.1.1 yields

$$
\begin{aligned}
\frac{1}{c^{2}} \underline{r}_{\varepsilon}^{2}(s) \mathrm{d} \sigma^{2} \leq\left(\left(1-\chi_{\varepsilon}(s)\right) r_{\varepsilon}^{2}(s)\right. & \left.+\frac{1}{c^{2}} \chi_{\varepsilon}(s) s^{2}\right) \mathrm{d} \sigma^{2} \leq h_{(\varepsilon, s, \cdot)}^{i} \leq \\
& \leq\left(\left(1-\chi_{\varepsilon}(s)\right) r_{\varepsilon}^{2}(s)+c^{2} \chi_{\varepsilon}(s) s^{2}\right) \mathrm{d} \sigma^{2} \leq c^{2} \bar{r}_{\varepsilon}^{2}(s) \mathrm{d} \sigma^{2}
\end{aligned}
$$

which implies the existence of another constant $c^{\prime}$ (again by Lemma 4.1.2) such that

$$
\frac{1}{c^{\prime}} \underline{r}_{\varepsilon}^{d-1}(s) \leq\left(\operatorname{det} h_{(\varepsilon, s, \cdot)}^{i}\right)^{\frac{1}{2}} \leq c^{\prime} \bar{r}_{\varepsilon}^{d-1}(s) .
$$

We denote the completion of $X \backslash\left\{x_{1}, \ldots, x_{2 r}\right\}$ together with the modified metrics $g_{(\varepsilon, s, \sigma)}^{i}=$ $\mathrm{d} s_{s}^{2}+h_{(\varepsilon, s, \sigma)}$ near $x^{i}$ by $M_{\varepsilon}$. Note that $X_{\varepsilon}$ is embedded in $M_{\varepsilon}$ and that the boundary of $M_{\varepsilon}$ has 


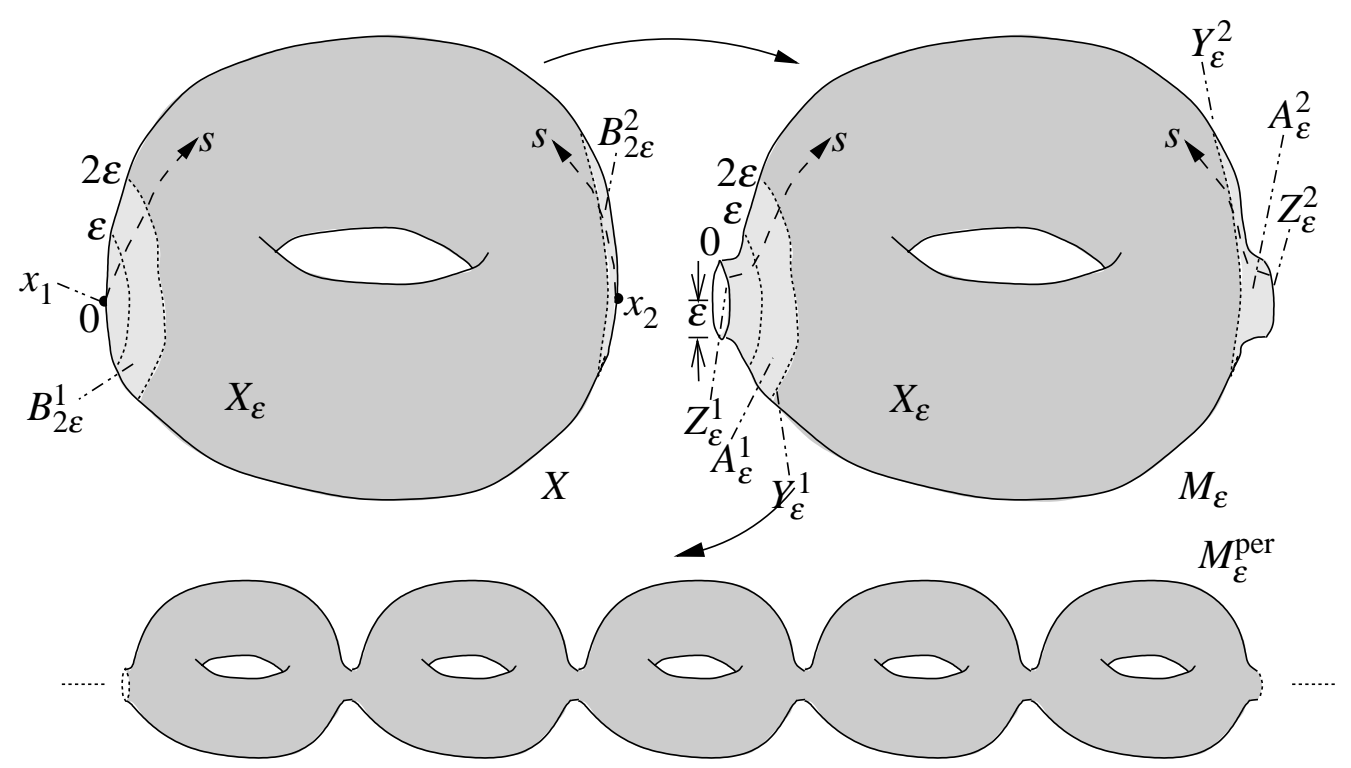

Figure 4.2: Construction of the periodic manifold $M_{\varepsilon}^{\text {per }}$. We start with the compact manifold $X$, modify $X$ in a neighbourhood of $x_{1}, \ldots, x_{2 r}$. Here, the period cell $M_{\varepsilon}$ has only $r=1$ pairs of cylindrical ends. Finally we glue the periodic manifold $M_{\varepsilon}^{\text {per }}$ from copies of the period cell.

$2 r$ new disjoint components $Z_{\varepsilon}^{1}, \ldots Z_{\varepsilon}^{2 r}$, each of them isometric to the sphere of radius $\varepsilon$. Let $A_{\varepsilon}^{i}$ be the part of the manifold $M_{\varepsilon}$ near $x_{i}$ given in polar coordinates by $[0,2 \varepsilon] \times \mathbb{S}$. Finally, we set $A_{\varepsilon}=A_{\varepsilon}^{1} \cup \cdots \cup A_{\varepsilon}^{2 r}, Y_{\varepsilon}=Y_{\varepsilon}^{1} \cup \cdots \cup Y_{\mathcal{\varepsilon}}^{2 r}$ and $Z_{\varepsilon}=Z_{\varepsilon}^{1} \cup \cdots \cup Z_{\mathcal{\varepsilon}}^{2 r}$ (see Figure 4.2).

Remember that $\Gamma$ is a finitely generated abelian group with $r$ generators $e_{1}, \ldots, e_{r}$. We suppose that $\Gamma$ has at least one infinite generator. Furthermore let $\gamma M_{\varepsilon}$ be an isometric copy of $M_{\varepsilon}$ with identification $x \mapsto \gamma \cdot x$ for each $\gamma \in \Gamma$. We construct a new manifold $M_{\varepsilon}^{\text {per }}$ by identifying $\gamma Z_{\varepsilon}^{2 i-1}$ with $e_{i} \gamma Z_{\varepsilon}^{2 i}$ for each $\gamma \in \Gamma$ and $i=1, \ldots, r$ (see Figure 4.2). Since in a neighbourhood of $Z_{\varepsilon}^{i}$ the manifold is isometric to a cylinder of radius $\varepsilon$, we can choose a smooth atlas and a smooth metric of the glued manifold $M_{\varepsilon}^{\text {per }}$. We therefore obtain a (non-compact) manifold which admits a cocompact $\Gamma$-action as in Chapter 3, so Floquet theory applies. The manifold $M_{\varepsilon}$ is a period cell for $M_{\varepsilon}^{\text {per }}$.

Note that or a $d$-dimensional manifold $M_{\varepsilon}^{\text {per }}$ a finitely generated group $\Gamma$ with $r$ generators (e. g. $\Gamma=\mathbb{Z}^{r}$ ) exists which acts cocompactly on $M_{\varepsilon}^{\text {per }}$. Thus the dimension of periodicity is independent of the dimension of the manifold. This is in contrast to the flat periodic case $M_{\varepsilon}^{\text {per }}=\mathbb{R}^{d}$ where the group action must have $r=d$ generators to obtain a cocompact action.

\subsection{Convergence of the eigenvalues}

Floquet theory allows us to analyse the spectrum of the periodic manifold $M_{\varepsilon}^{\text {per }}$ by analysing the spectrum of the $\theta$-periodic Laplacian on the period cell $M_{\varepsilon}$. We impose $\theta$-periodic boundary conditions between $Z_{\varepsilon}^{2 i-1}$ and $Z_{\mathcal{\varepsilon}}^{2 i}$ for each $i=1, \ldots, r$. If $\partial M_{\varepsilon}$ contains more components (thus components of $\partial X$ ) we leave the boundary conditions on $X$ unchanged.

Now we are able to state our first main result: 
4.3.1. Theorem. Let $\Gamma$ be an abelian group with $r$ generators. Let $M_{\varepsilon}^{\mathrm{per}}$ be constructed as above by glueing together $\Gamma$ copies of a compact manifold $M_{\mathcal{\varepsilon}}$. Here, $M_{\varepsilon}$ is obtained by taking away $2 r$ points from $X$ and changing the metric such that the ends look like small cylinders.

Then the $k$-th Dirichlet as well as the $k$-th Neumann eigenvalue (denoted by $\lambda_{k}^{\mathrm{D} / \mathrm{N}}\left(M_{\varepsilon}\right)$ ) converges to the $k$-th eigenvalue $\lambda_{k}(X)$ of $X$ as $\varepsilon \rightarrow 0$.

In particular, the $k$-th $\theta$-periodic eigenvalue $\lambda_{k}^{\theta}\left(M_{\varepsilon}\right)$ converges uniformly in $\theta \in \hat{\Gamma}$ to the $k$-th eigenvalue $\lambda_{k}(X)$ of $X$ as $\varepsilon \rightarrow 0$.

Applying the Floquet theory we obtain the first example of a periodic manifold with gaps in its spectrum:

4.3.2. Corollary. For $\varepsilon$ small enough the $k$-th band $B_{k}\left(\Delta_{M_{\varepsilon}^{\text {per }}}\right)$ and the $(k+1)$-st band $B_{k+1}\left(\Delta_{M_{\varepsilon}^{\text {per }}}\right)$ do not overlap, provided $\lambda_{k}(X)<\lambda_{k+1}(X)$. In particular, for given $N \in \mathbb{N}$ there exists $\varepsilon_{0}>0$ such that the periodic operator $\Delta_{M_{\varepsilon}^{\text {per }}}$ has at least $N$ gaps for $0<\varepsilon<\varepsilon_{0}$.

To show the convergence of the Dirichlet resp. Neumann eigenvalues $\lambda_{k}^{\mathrm{D} / \mathrm{N}}\left(M_{\varepsilon}\right)$ on $M_{\varepsilon}$ for $\varepsilon \rightarrow 0$ we will enclose them by eigenvalues of Laplacians with Dirichlet or Neumann boundary conditions on $X_{\varepsilon}$. Therefore we need the convergence of the spectrum of these operators. Fortunately, these results are already in the literature, so we will only quote the results.

4.3.3. Theorem. The spectrum of the Dirichlet Laplacian on $X_{\varepsilon}$ converges to the spectrum of the Laplacian on $X$, i.e., $\lim _{\mathcal{\varepsilon} \rightarrow 0} \lambda_{k}^{\mathrm{D}}\left(X_{\mathcal{\varepsilon}}\right)=\lambda_{k}(X)$.

Proof. (see [CF78])

4.3.4. Theorem. The spectrum of the Neumann Laplacian on $X_{\varepsilon}$ converges to the spectrum of the Laplacian on $X$, i.e., $\lim _{\mathcal{\varepsilon} \rightarrow 0} \lambda_{k}^{\mathrm{N}}\left(X_{\mathcal{\varepsilon}}\right)=\lambda_{k}(X)$.

Proof. (see [Ann87])

Proof of Theorem 4.3.1. First we give an estimate from above for the Dirichlet eigenvalues $\lambda_{k}^{\mathrm{D}}\left(M_{\varepsilon}\right)$ of the quadratic form $q_{M_{\varepsilon}}^{\mathrm{D}}$. This is easily done by the embedding

$$
\operatorname{dom}\left(q_{X_{\varepsilon}}^{\mathrm{D}}\right)=\stackrel{\mathscr{H}}{ }^{1}\left(X_{\varepsilon}\right) \hookrightarrow \stackrel{\mathscr{H}}{ }^{1}\left(M_{\varepsilon}\right)=\operatorname{dom}\left(q_{M_{\varepsilon}}^{\mathrm{D}}\right)
$$

for the domains of the quadratic forms. The Min-max Principle yields

$$
\lambda_{k}^{\mathrm{D}}\left(X_{\varepsilon}\right) \geq \lambda_{k}^{\mathrm{D}}\left(M_{\varepsilon}\right)
$$

For the estimate from below we need our Main Lemma (formally, the Main Lemma (Theorem 1.4.2) can also be applied to obtain the estimate from above). We set

$$
\begin{array}{lll}
\mathscr{H}_{\varepsilon}:=L_{2}\left(M_{\varepsilon}\right), & \operatorname{dom} q_{\varepsilon}:=\mathscr{H}^{1}\left(M_{\varepsilon}\right), & q_{\varepsilon}:=q_{M_{\varepsilon}}^{\mathrm{N}}, \\
\mathscr{H}_{\varepsilon}^{\prime}:=L_{2}\left(X_{\varepsilon}\right), & \operatorname{dom} q_{\varepsilon}^{\prime}:=\mathscr{H}^{1}\left(X_{\varepsilon}\right), & q_{\varepsilon}^{\prime}:=q_{X_{\varepsilon}}^{\mathrm{N}}
\end{array}
$$

and the operator $\Phi_{\varepsilon}$ is just the restriction of $u_{\varepsilon}$ to $X_{\varepsilon}$, e.g. $\Phi_{\varepsilon} u_{\varepsilon}:=\left.u_{\varepsilon}\right|_{X_{\varepsilon}}$.

We have to verify the conditions of Theorem 1.4.2 resp. of Corollary 1.4.4. 
Condition 1 resp. (1.15) is satisfied if

$$
\left|\left\|\Phi_{\varepsilon} u_{\varepsilon}\right\|^{2}-\left\|u_{\varepsilon}\right\|^{2}\right|=\int_{M_{\varepsilon} \backslash X_{\varepsilon}}\left|u_{\varepsilon}\right|^{2}=\int_{A_{\varepsilon}}\left|u_{\varepsilon}\right|^{2} \leq \omega(\varepsilon)\left\|u_{\varepsilon}\right\|_{q_{M_{\varepsilon}}^{\mathrm{N}}}^{2}
$$

with a positive function $\omega(\varepsilon) \rightarrow 0$ as $\varepsilon \rightarrow 0$. The existence of $\omega(\varepsilon)$ will be shown Theorem 4.4.1.

Condition 2 resp. (1.16) is trivially satisfied because of

$$
q_{M_{\varepsilon}}^{\mathrm{N}}\left(u_{\varepsilon}\right)=\int_{M_{\varepsilon}}\left|\mathrm{d} u_{\varepsilon}\right|^{2} \geq \int_{X_{\varepsilon}}\left|\mathrm{d} u_{\varepsilon}\right|^{2}=q_{X_{\varepsilon}}^{\mathrm{N}}\left(\Phi_{\varepsilon} u_{\varepsilon}\right) .
$$

Note the advantage of not having to prove the convergence of the quadratic forms.

Condition 3 is satisfied by Estimate (4.5) and Theorem 4.3.3.

Finally, Theorem 1.4.2 implies

$$
\lambda_{k}^{\mathrm{N}}\left(X_{\varepsilon}\right)-\delta_{k}(\varepsilon) \leq \lambda_{k}^{\mathrm{N}}\left(M_{\varepsilon}\right)
$$

with $\delta_{k}(\varepsilon) \rightarrow 0$ as $\varepsilon \rightarrow 0$.

Estimates (4.5) and (4.6) together with the Dirichlet-Neumann enclosure (see (3.12)) show

$$
\lambda_{k}^{\mathrm{N}}\left(X_{\varepsilon}\right)-\delta_{k}(\varepsilon) \leq \lambda_{k}^{\mathrm{N}}\left(M_{\varepsilon}\right) \leq \lambda_{k}^{\theta}\left(M_{\varepsilon}\right) \leq \lambda_{k}^{\mathrm{D}}\left(M_{\varepsilon}\right) \leq \lambda_{k}^{\mathrm{D}}\left(X_{\varepsilon}\right) .
$$

Therefore Theorem 4.3.3 and Theorem 4.3.4 imply the convergence of the Dirichlet, Neumann and $\theta$-periodic $k$-th eigenvalue on $M_{\varepsilon}$ to the $k$-th eigenvalue on $X$. Note that the convergence is uniform in $\theta \in \hat{\Gamma}$ since $\delta_{k}(\varepsilon)$ does not depend on $\theta$.

\subsection{Estimate on the cylindrical ends}

The following theorem, based on articles by C. Anné (cf. [Ann87], [Ann94, Lemme A] and [Ann99]), gives us two important estimates on the boundary $Z_{\varepsilon}$ resp. the junction $A_{\varepsilon}$. The estimate over the boundary is an intermediate result which will be needed in Chapter 5 (when glueing a long thin cylinder to $M_{\varepsilon}$ ).

4.4.1. Theorem. There exists a positive function $\omega(\varepsilon)$ converging to 0 as $\varepsilon \rightarrow 0$ such that

$$
\int_{Z_{\varepsilon}}\left|u_{\mathcal{\varepsilon}}\right|^{2}, \int_{A_{\varepsilon}}\left|u_{\mathcal{\varepsilon}}\right|^{2} \leq \omega(\varepsilon) \int_{M_{\varepsilon}}\left(\left|u_{\varepsilon}\right|^{2}+\left|\mathrm{d} u_{\varepsilon}\right|^{2}\right)
$$

for all $u_{\varepsilon} \in \mathscr{H}^{1}\left(M_{\varepsilon}\right)$.

Note that $\omega(\varepsilon)$ only depends on the geometry of $X$ near $x_{i}$.

Proof. We prove the result for the components $Z_{\varepsilon}^{i}$ of $Z_{\varepsilon}$ resp. $A_{\varepsilon}^{i}$ of $A_{\varepsilon}$ separatly. Suppose $u_{\varepsilon} \in C^{\infty}\left(M_{\varepsilon}\right)$ with $u_{\varepsilon}\left(\varepsilon_{0}, \sigma\right)=0$ for all $\sigma \in \mathbb{S}$. First we show an $L_{2}$-estimate over $A_{\varepsilon, s}^{i}:=$ $\{s\} \times \mathbb{S} \subset A_{\varepsilon}^{i}$ with its induced metric $h_{(\varepsilon, s, \cdot)}$.

Applying the Cauchy-Schwarz Inequality together with Estimate (4.4) yields

$$
\begin{aligned}
\left|u_{\varepsilon}(s, \sigma)\right|^{2} & =\left|\int_{s}^{\varepsilon_{0}} \partial_{t} u_{\varepsilon}(t, \sigma) \mathrm{d} t\right|^{2} \\
& \leq c^{\prime} \int_{s}^{\varepsilon_{0}}\left(\frac{c}{\underline{r}_{\varepsilon}(t)}\right)^{d-1} \mathrm{~d} t \int_{s}^{\varepsilon_{0}}\left|\partial_{t} u_{\varepsilon}(t, \sigma)\right|^{2}\left(\operatorname{det} h_{(\varepsilon, t, \sigma)}\right)^{\frac{1}{2}} \mathrm{~d} t .
\end{aligned}
$$


If we integrate over $\sigma \in \mathbb{S}^{d-1}$ we obtain

$$
\begin{aligned}
\int_{A_{\varepsilon, s}^{i}}\left|u_{\varepsilon}\right|^{2} & =\int_{\mathbb{S}}\left|u_{\varepsilon}(s, \sigma)\right|^{2}\left(\operatorname{det} h_{(\varepsilon, s, \sigma)}\right)^{\frac{1}{2}} \mathrm{~d} \sigma \\
& \leq \underbrace{c^{\prime}\left(\bar{r}_{\varepsilon}(s)\right)^{d-1} \int_{s}^{\varepsilon_{0}}\left(\frac{c}{\underline{r}_{\varepsilon}(t)}\right)^{d-1} \mathrm{~d} t}_{=: \omega_{s}(\varepsilon)} \int_{M_{\varepsilon}}\left|\mathrm{d} u_{\varepsilon}\right|^{2} .
\end{aligned}
$$

We have constructed $\underline{r}_{\varepsilon}$ resp. $\bar{r}_{\varepsilon}$ such that $\underline{r}_{\varepsilon}(t)=\varepsilon$ for $0 \leq t \leq \varepsilon$ and $\underline{r}_{\varepsilon}(t)=t$ for $\varepsilon \leq t \leq \varepsilon_{0}$ resp. $\bar{r}_{\varepsilon}(s) \leq 2 \varepsilon$ for $0 \leq s \leq \varepsilon_{0}$. Therefore

$$
\omega_{s}(\varepsilon) \leq c^{\prime}(2 \varepsilon)^{d-1}\left(\int_{0}^{\varepsilon} \frac{1}{\varepsilon^{d-1}} \mathrm{~d} t+\int_{\varepsilon}^{\varepsilon_{0}} \frac{1}{t^{d-1}} \mathrm{~d} t\right)=: \omega(\varepsilon)
$$

where $\omega(\varepsilon)$ converges to 0 as $\varepsilon \rightarrow 0$. If we set $s=0$ we obtain the statement for $Z_{\varepsilon}^{i}=A_{\varepsilon, 0}^{i}$. Furthermore,

$$
\int_{A_{\varepsilon}^{i}}\left|u_{\varepsilon}\right|^{2}=\int_{0}^{2 \varepsilon} \int_{A_{\varepsilon, s}^{i}}\left|u_{\varepsilon}\right|^{2} \leq 2 \varepsilon \omega(\varepsilon) \int_{M \varepsilon}\left|\mathrm{d} u_{\varepsilon}\right|^{2} .
$$

If $u\left(\varepsilon_{0}, \sigma\right) \neq 0$ we choose a cut-off function (which is independent of $\varepsilon$ !). If $u \in \mathscr{H}^{1}\left(M_{\varepsilon}\right)$ we apply an approximation argument. 


\section{Periodic manifold joined by cylinders}

In this chapter we construct another class of periodic manifolds with gaps in the spectrum. This time, we admit long thin cylinders of fixed length between each modified manifold. Roughly speaking, we join $\Gamma$ copies of a compact manifold $X$ by long thin cylinders. As before, we obtain gaps in the spectrum if we let the radius of these cylinders tend to 0 .

\subsection{Construction of the period cell}

Let $\Gamma$ be an abelian group with $r$ generators. Suppose that $M_{\varepsilon}$ is the period cell constructed in the previous chapter, i.e., $M_{\varepsilon}$ is obtained by taking away $2 r$ points $x_{i}$ from $X$ and modifying the metric in a neighbourhood of each point $x_{i}$ such that small cylindrical ends of radius $\varepsilon$ arise at each point $x_{i}$ (see Section 4.2). Suppose that $L_{i}>0$ for $i=1, \ldots, r$ and set $I^{i}:=\left[0, L_{i}\right]$. Now for each $i=1, \ldots, r$ we glue one end of the cylinder $C_{\varepsilon}^{i}:=I^{i} \times \mathbb{S}$ with metric $\mathrm{d} s^{2}+\varepsilon^{2} \mathrm{~d} \sigma^{2}$ to the component $Z_{\varepsilon}^{2 i-1}$ of the boundary of $M_{\mathcal{\varepsilon}}$. We call the resulting compact manifold $\widetilde{M}_{\varepsilon}$ (see Figure 5.1). Let $C_{\varepsilon}$ be the (disjoint) union of $C_{\varepsilon}^{1}, \ldots, C_{\varepsilon}^{r}$ and let $I$ be the (disjoint) union of $I^{1}, \ldots, I^{r}$.

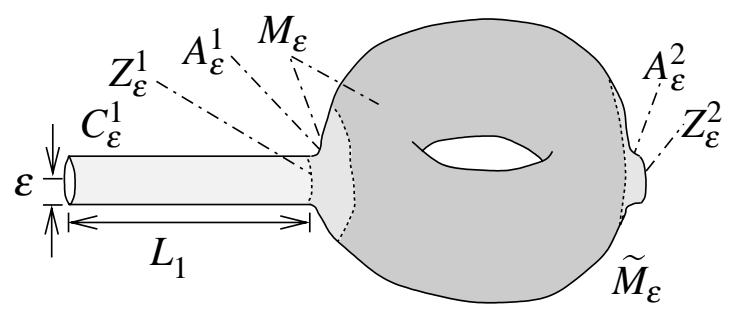

Figure 5.1: Here, the period cell $\widetilde{M}_{\varepsilon}$ is obtained from glueing the cylinder $C_{\varepsilon}^{1}$ of lenght $L_{1}>0$ and radius $\varepsilon>0$ to the period cell $M_{\varepsilon}$ constructed in Figure 4.2.

We obtain the periodic manifold $\widetilde{M}_{\varepsilon}^{\text {per }}$ in the same way as in the last chapter by glueing together $\Gamma$ copies of the period cell $\widetilde{M}_{\varepsilon}$ (see Figure 0.3 on page 5).

\subsection{Convergence of the eigenvalues}

As before we use Floquet theory to analyse the spectrum of the periodic manifold $\widetilde{M}_{\varepsilon}^{\text {per }}$ by analysing the spectrum of the $\theta$-periodic Laplacian on the period cell $\widetilde{M}_{\mathcal{\varepsilon}}$. We impose $\theta$ periodic boundary conditions between $Z_{\mathcal{\varepsilon}}^{2 i}$ and the free end of $C_{\mathcal{\varepsilon}}^{i}$ for each $i=1, \ldots, r$. If $\partial X \neq \emptyset$ we leave the boundary conditions on $X$ unchanged. 
We denote by $\lambda_{k}^{\mathrm{D}}(X \dot{\cup} I)=\lambda_{k}^{\mathrm{D}}\left(X \dot{\cup} I^{1} \dot{\cup} \ldots \dot{\cup} I^{r}\right)$ the spectrum of the Dirichlet Laplacian on $X \dot{\cup} I$ (note that $X \dot{\cup} I$ consists of $r+1$ components). The spectrum of this manifold is the union of the spectra of its components, thus $\lambda_{k}^{\mathrm{D}}(X \cup \dot{\cup} I)$ is just a rearrangement in increasing order and repeated according to multiplicity of all eigenvalues $\lambda_{m}(X), \lambda_{m_{1}}^{\mathrm{D}}\left(I^{1}\right), \ldots, \lambda_{m_{r}}^{\mathrm{D}}\left(I^{r}\right)$.

In the same way, we denote by $\lambda_{k}^{\mathrm{DN}}(X \dot{\cup} I)$ the spectrum of the Laplacian on $X$ and the spectrum of the Dirichlet-Neumann Laplacian on $I$, i.e., we pose Dirichlet boundary conditions at 0 and Neumann boundary condions at $L_{i}$ on each interval $I^{i}$.

Our second main result is the following:

5.2.1. Theorem. Let $\Gamma$ be an abelian group with $r$ generators. Let $\widetilde{M}_{\varepsilon}^{\text {per }}$ be constructed as above by glueing together $\Gamma$ copies of a compact manifold $\widetilde{M}_{\mathcal{\varepsilon}}$. Here, $\widetilde{M}_{\mathcal{\varepsilon}}$ is obtained by glueing $r$ cylinders of lenght $L_{i}$ and radius $\varepsilon$ to the component $Z_{\mathcal{\varepsilon}}^{2 i-1}$ of $\partial M_{\mathcal{\varepsilon}}$.

Then the $k$-th Dirichlet resp. $\theta$-periodic eigenvalue $\lambda_{k}^{\mathrm{D}}\left(\widetilde{M}_{\varepsilon}\right)$ resp. $\lambda_{k}^{\theta}\left(\widetilde{M}_{\varepsilon}\right)$ converges (uniformly in $\theta \in \hat{\Gamma})$ to the $k$-th Dirichlet eigenvalue $\lambda_{k}^{\mathrm{D}}(X \dot{\cup} I)$ of $X \cup \dot{\cup} I$ as $\varepsilon \rightarrow 0$. The $k$-th Neumann eigenvalue $\lambda_{k}^{\mathrm{N}}\left(\widetilde{M}_{\varepsilon}\right)$ converges to the $k$-th Dirichlet-Neumann eigenvalue $\lambda_{k}^{\mathrm{ND}}(X \dot{\cup} I)$ of $X \dot{\cup} I$ as $\varepsilon \rightarrow 0$.

Again, applying Floquet theory we obtain:

5.2.2. Corollary. For $\varepsilon$ small enough the $k$-th band $B_{k}\left(\Delta_{\widetilde{M}_{\varepsilon}^{\text {per }}}\right)$ and the $(k+1)$-st band $B_{k+1}\left(\Delta_{\widetilde{M}_{\varepsilon}^{\text {per }}}\right)$ do not overlap, provided that $\lambda_{k}(X \cup \dot{\cup} I)<\lambda_{k+1}(X \cup \dot{\cup} I)$. In particular, for given $N \in \mathbb{N}$ there exists $\varepsilon>0$ such that the periodic operator $\Delta_{\widetilde{M}_{\varepsilon}^{\mathrm{per}}}$ has at least $N$ gaps.

Note that this result is in some sense less satisfactory than the result of Chapter 4 because the limit spectrum contains more points. If one is interested in gaps it would be better to have a limit spectrum with few points.

Nevertheless, this example shows another phenomena. The volume of the $\varepsilon$-depending part $C_{\varepsilon} \cup A_{\varepsilon}$ is of the order $O\left(\varepsilon^{d-1}\right)$ in contrast to the example of the preceding chapter. There, we had $\operatorname{vol}\left(A_{\varepsilon}\right)=O\left(\varepsilon^{d}\right)$. Therefore, the periodic manifold $\widetilde{M}_{\varepsilon}^{\text {per }}$ leads to the conjecture that the separation procedure producing gaps only needs a surface term (i.e., a submanifold of dimension $d-1$ ) whose volume converges to 0 .

To show the convergence of the $\theta$-periodic resp. Dirichlet eigenvalues we will enclose them by Laplacians with Dirichlet or Neumann boundary conditions on $X_{\varepsilon}$ and Dirichlet boundary condition on $C_{\varepsilon}$. We further need the convergence of the spectrum of the cylinders. One would expect that the spectrum of the cylinder $C_{\varepsilon}^{i}$ converges to the spectrum of the interval $I^{i}$ as $\varepsilon \rightarrow 0$ since $C_{\varepsilon}^{i}$ collapses to $I^{i}$. This is indeed the case:

5.2.3. Theorem. The spectrum of the Dirichlet resp. Dirichlet-Neumann Laplacian on $C_{\varepsilon}^{i}$ converges to the spectrum of the Dirichlet resp. Dirichlet-Neumann Laplacian on $I^{i}=\left[0, L_{i}\right]$, e.g., $\lim _{\varepsilon \rightarrow 0} \lambda_{k}^{\mathrm{D}}\left(C_{\varepsilon}^{i}\right)=\lambda_{k}^{\mathrm{D}}\left(I^{i}\right)$ resp. $\lim _{\varepsilon \rightarrow 0} \lambda_{k}^{\mathrm{DN}}\left(C_{\varepsilon}^{i}\right)=\lambda_{k}^{\mathrm{DN}}\left(I^{i}\right)$.

Proof. We have

$$
\lambda_{k_{1}, k_{2}}^{\mathrm{D}}\left(C_{\varepsilon}^{i}\right)=\frac{1}{\varepsilon^{2}} \lambda_{k_{1}}(\mathbb{S})+\lambda_{k_{2}}^{\mathrm{D}}\left(I^{i}\right)
$$

The result follows by rearranging these eigenvalues as $\varepsilon \rightarrow 0$. 
In Chapter 8 we need estimates on the limit spectrum:

5.2.4. Lemma. We have $\lambda_{k}^{\mathrm{D}}\left(I^{i}\right)=\frac{\pi^{2}}{\left(L_{i}\right)^{2}} k^{2}$ and $\lambda_{k}^{\mathrm{DN}}\left(I^{i}\right)=\frac{\pi^{2}}{\left(L_{i}\right)^{2}}\left(k-\frac{1}{2}\right)^{2}, k \in \mathbb{N}$. In particular, $\lambda_{k}^{\mathrm{D}}\left(I^{i}\right)<\lambda_{k+1}^{\mathrm{DN}}\left(I^{i}\right)$ and $\lambda_{k}^{\mathrm{D}}(X \dot{\cup} I) \leq \lambda_{k+1}^{\mathrm{DN}}(X \dot{\cup} I)$ with strict inequality if $\lambda_{k}^{\mathrm{D}}(X \dot{\cup} I)$ and $\lambda \lambda_{k+1}^{\mathrm{DN}}(X \dot{\cup} I)$ are not in the spectrum of $\Delta_{X}$.

Now we prove the main result of this chapter.

Proof of Theorem 5.2.1. We call $\lambda_{k}^{\mathrm{DD}}\left(X_{\varepsilon} \dot{\cup} C_{\varepsilon}\right)$ resp. $\lambda_{k}^{\mathrm{ND}}\left(X_{\varepsilon} \dot{\cup} C_{\varepsilon}\right)$ the eigenvalues, ordered according to multiplicity, of the quadratic form $q_{X_{\varepsilon}}^{\mathrm{D}} \oplus q_{C_{\varepsilon}}^{\mathrm{D}}$ resp. $q_{X_{\varepsilon}}^{\mathrm{N}} \oplus q_{C_{\varepsilon}}^{\mathrm{D}}$ corresponding to the Laplacian on $X_{\mathcal{E}} \cup C_{\varepsilon}$. Note that these eigenvalues converge to points of the union of the spectra of $q_{X}$ and $q_{I}^{\mathrm{D}}$ (see Theorems 4.3.3, 4.3.4 and 5.2.3).

We first give an estimate from above for the $\theta$-periodic and Dirichlet eigenvalues. The embedding

$$
\mathscr{\mathscr { H }}^{1}\left(X_{\varepsilon}\right) \oplus \mathscr{\mathscr { H }}^{1}\left(C_{\varepsilon}\right) \hookrightarrow \stackrel{\mathscr{H}}{1}^{1}\left(\widetilde{M}_{\varepsilon}\right) \hookrightarrow \mathscr{H}_{\theta}^{1}\left(\widetilde{M}_{\varepsilon}\right)
$$

for the domains of the quadratic forms with Dirichlet resp. $\theta$-periodic boundary conditions yields the inequality

$$
\lambda_{k}^{\mathrm{DD}}\left(X_{\varepsilon} \dot{\cup} C_{\varepsilon}\right) \geq \lambda_{k}^{\mathrm{D}}\left(\widetilde{M}_{\varepsilon}\right) \geq \lambda_{k}^{\theta}\left(\widetilde{M}_{\varepsilon}\right)
$$

via the Min-max Principle.

For the estimate from below we also apply our Main Lemma with

$$
\begin{array}{lll}
\mathscr{H}_{\varepsilon}:=L_{2}\left(\widetilde{M}_{\varepsilon}\right), & \operatorname{dom} q_{\varepsilon}:=\mathscr{H}_{\theta}^{1}\left(\widetilde{M}_{\varepsilon}\right), & q_{\varepsilon}:=q_{\widetilde{M}_{\varepsilon}}^{\theta}, \\
\mathscr{H}_{\varepsilon}^{\prime}:=L_{2}\left(X_{\varepsilon}\right) \oplus L_{2}\left(C_{\varepsilon}\right), & \operatorname{dom} q_{\varepsilon}^{\prime}:=\mathscr{H}^{1}\left(X_{\varepsilon}\right) \oplus \mathscr{H}^{1}\left(C_{\varepsilon}\right), & q_{\varepsilon}^{\prime}:=q_{X_{\varepsilon}}^{\mathrm{N}} \oplus q_{C_{\varepsilon}}^{\mathrm{D}} .
\end{array}
$$

We further set $u_{\varepsilon}=v_{\varepsilon} \oplus w_{\varepsilon}$ and $\Phi_{\varepsilon} u_{\varepsilon}:=v_{\varepsilon} \oplus\left(w_{\varepsilon}-H_{\varepsilon} u_{\varepsilon}\right)$ where $h_{\varepsilon}:=H_{\varepsilon} u_{\varepsilon}$ is the harmonic function $\left(\Delta_{C_{\varepsilon}} h_{\varepsilon}=0\right)$ with boundary condition $h_{\varepsilon} \uparrow_{\partial C_{\varepsilon}}=u_{\varepsilon} \uparrow_{\partial C_{\varepsilon}}$. By construction we have $f_{\varepsilon}=w_{\varepsilon}-h_{\varepsilon} \in \mathscr{\mathscr { H }}^{1}\left(C_{\varepsilon}\right)$. Note that $h_{\varepsilon}$ exists and that $h_{\varepsilon}$ is unique by Theorem 3.5.3. The idea of using the harmonic extension on the cylinder is due to [Ann87].

Now, we have to verify the conditions of the Main Lemma (Theorem 1.4.2). In order to show Condition 1 we estimate

$$
\begin{aligned}
\left|\left\|\Phi_{\varepsilon} u_{\varepsilon}\right\|^{2}-\left\|u_{\varepsilon}\right\|^{2}\right| & \leq \int_{A_{\varepsilon}}\left|u_{\varepsilon}\right|^{2}+\int_{C_{\varepsilon}}\left(\left|w_{\varepsilon}-h_{\varepsilon}\right|^{2}-\left|w_{\varepsilon}\right|^{2}\right) \\
& \leq\left\|u_{\varepsilon}\right\|_{L_{2}\left(A_{\varepsilon}\right)}^{2}+2\left\|u_{\varepsilon}\right\|_{L_{2}\left(C_{\varepsilon}\right)}\left\|h_{\varepsilon}\right\|_{L_{2}\left(C_{\varepsilon}\right)}+\left\|h_{\varepsilon}\right\|_{L_{2}\left(C_{\varepsilon}\right)}^{2} .
\end{aligned}
$$

Since we already have proven $\left\|u_{\varepsilon}\right\|_{L_{2}\left(A_{\varepsilon}\right)}^{2} \rightarrow 0$ for every $q_{M_{\varepsilon}}^{\theta}$-bounded family $\left(u_{\varepsilon}\right)_{\varepsilon}$ as $\varepsilon \rightarrow 0$ in Theorem 4.4.1 it is sufficient to show the convergence $\left\|h_{\varepsilon}\right\|_{L_{2}\left(C_{\varepsilon}\right)}^{2} \rightarrow 0$ uniformly in $\theta$. This will be done in the next section.

In order to verify Condition 2 we need Lemma 2.3.11 which is one reason why we have chosen the harmonic extension on the cylinder. In short, it says that Dirichlet functions minimize the energy integral when adding harmonic functions, i.e.,

$$
\left\|\mathrm{d} w_{\varepsilon}-\mathrm{d} h_{\varepsilon}\right\|_{L_{2}\left(T^{*} C_{\varepsilon}\right)}^{2} \leq\left\|\mathrm{d} w_{\varepsilon}\right\|_{L_{2}\left(T^{*} C_{\varepsilon}\right)}^{2} .
$$


This yields a proof of condition 2:

$$
q_{\varepsilon}^{\prime}\left(\Phi_{\varepsilon} u_{\varepsilon}\right)=q_{X_{\varepsilon}}^{\mathrm{N}}\left(v_{\varepsilon}\right)+q_{C_{\varepsilon}}^{\mathrm{D}}\left(w_{\varepsilon}-h_{\varepsilon}\right) \leq q_{X_{\varepsilon}}^{\mathrm{N}}\left(v_{\varepsilon}\right)+q_{C_{\varepsilon}}^{\mathrm{D}}\left(w_{\varepsilon}\right)=q_{\widetilde{M}_{\varepsilon}}^{\theta}\left(u_{\varepsilon}\right)=q_{\varepsilon}\left(u_{\varepsilon}\right) .
$$

Condition 3 is satisfied because of Estimate (5.1) and the convergence of the Dirichlet eigenvalues (see Theorem 4.3.3 and Theorem 5.2.3). The constant in condition 3 is also independent of $\theta$.

Theorem 1.4.2 yields

$$
\lambda_{k}^{\mathrm{ND}}\left(X_{\varepsilon} \dot{\cup} C_{\varepsilon}\right)-\delta_{k}(\varepsilon) \leq \lambda_{k}^{\theta}\left(\widetilde{M}_{\varepsilon}\right)
$$

for some $\delta_{k}(\varepsilon) \rightarrow 0$ as $\varepsilon \rightarrow 0$ uniformly in $\theta$.

Estimates (5.1) and (5.2) together with Theorem 4.3.3, Theorem 4.3.4 and Theorem 5.2.3 imply the convergence of the $k$-th $\theta$-periodic resp. Dirichlet eigenvalue to the $k$-th eigenvalue of $X \dot{\cup} I$ with Dirichlet boundary conditions on the interval $I$.

We will not prove the convergence $\lambda_{k}^{\mathrm{N}}\left(\widetilde{M}_{\varepsilon}\right) \rightarrow \lambda_{k}^{\mathrm{DN}}(X \cup \dot{\cup} I)$ since it can be done in essentially the same way.

\subsection{Estimate of the harmonic extension}

For the proof of the $L_{2}$-convergence of the harmonic extension on the cylinders we follow C. Anné [Ann87] and [Ann99].

In this context it is useful to work in an $\varepsilon$-independent Hilbert space. This will be achieved by the transformation

$$
\begin{aligned}
T_{\varepsilon}: L_{2}\left(C_{\varepsilon}\right) & \longrightarrow L_{2}(C) \\
u & \longmapsto \varepsilon^{\frac{d-1}{2}} u
\end{aligned}
$$

with $C=C_{1}$ being the cylinder $I \times \mathbb{S}$ (resp. the disjoint union of the cylinders $I^{i} \times \mathbb{S}$ ) with standard metric. Denote by $\partial_{t}$ and $d_{\mathbb{S}}$ the derivative with respect to $t \in I$ and $\sigma \in \mathbb{S}=\mathbb{S}^{d-1}$. The transformed quadratic form of the Laplacian on $C_{\varepsilon}$ is

$$
T_{*} q_{C_{\varepsilon}}(u)=q_{C_{\varepsilon}}\left(T_{\varepsilon}^{-1} u\right)=\int_{C}\left(\left|\partial_{t} u\right|^{2}+\frac{1}{\varepsilon^{2}}\left|\mathrm{~d}_{\mathbb{S}} u\right|^{2}\right)
$$

with the same domain $\operatorname{dom} T_{*} q_{C_{\varepsilon}}^{\mathrm{D}}=\operatorname{dom} q_{C_{\varepsilon}}^{\mathrm{D}}=\ddot{\mathscr{H}}^{1}(C)$ for each $\varepsilon$ if we have Dirichlet boundary conditions (the same statement holds for Dirichlet-Neumann boundary conditions). In the sequel we will not in general distinguish between the form $q_{C_{\varepsilon}}$ on $L_{2}\left(C_{\varepsilon}\right)$ and $T_{*} q_{C_{\varepsilon}}$ on $L_{2}(C)$.

We will prove the following theorem in several steps (see [Ann99]).

5.3.1. Theorem. The integral $\int_{C_{\varepsilon}}\left|H_{\varepsilon} u_{\varepsilon}^{\theta}\right|^{2}$ converges to 0 uniformly in $\theta$ for all $q_{C_{\varepsilon}}^{\theta}$-bounded families $\left(u_{\varepsilon}^{\theta}\right)_{\varepsilon, \theta}$ (also bounded in $\theta$ !).

We first show that a limit $h$ exists for subsequences of the bounded family.

5.3.2. Lemma. Let $\left(u_{\varepsilon}^{\theta}\right)_{\varepsilon, \theta}$ be a $q_{\widetilde{M}_{\varepsilon}}^{\theta}$-bounded family and $h_{\varepsilon}^{\theta}:=H_{\varepsilon} u_{\varepsilon}^{\theta}$. Then the families $\left(\left\|h_{\varepsilon}^{\theta}\right\|_{\mathscr{H}^{1}(C)}\right)_{\varepsilon, \theta}$ and $\left(q_{C_{\varepsilon}}\left(h_{\varepsilon}^{\theta}\right)\right)_{\varepsilon, \theta}$ are bounded (in $\varepsilon$ and $\theta$ ).

In particular, for all sequences $\left(\varepsilon_{m}\right)_{m}$ and $\left(\theta_{m}\right)_{m}$ with $\varepsilon_{m} \rightarrow 0$ we can find subsequences $\left(\varepsilon_{m_{n}}\right)_{n}$ and $\left(\theta_{m_{n}}\right)_{n}$ such that $h_{n}:=h_{\varepsilon_{m_{n}}}^{\theta_{m_{n}}}$ converges weakly in $\mathscr{H}^{1}(C)$ and strongly in $L_{2}(C)$ to an element $h \in \mathscr{H}^{1}(C)$. We will denote the subsequences also by $\left(\varepsilon_{n}\right)$ and $\left(\theta_{n}\right)$. 
Proof. Let $\left(u_{\varepsilon}^{\theta}\right)_{\varepsilon, \theta}$ be a family bounded by some constant $c>0$. Furthermore, let $\lambda_{1}^{\mathrm{D}}\left(C_{1}\right)>0$ be the first Dirichlet eigenvalue of the quadratic form $q_{C_{1}}^{\mathrm{D}}$. Set $w_{\varepsilon}^{\theta}=u_{\varepsilon}^{\theta} \uparrow_{C_{\varepsilon}}$. By construction of $h_{\varepsilon}^{\theta}$ we have $w_{\varepsilon}^{\theta}-h_{\varepsilon}^{\theta} \in \dot{\mathscr{H}}^{1}(C)$ and therefore

$$
\begin{aligned}
& \lambda_{1}^{\mathrm{D}}\left(C_{1}\right)\left\|w_{\varepsilon}^{\theta}-h_{\varepsilon}^{\theta}\right\|_{L_{2}(C)}^{2} \leq q_{C_{1}}^{\mathrm{D}}\left(w_{\varepsilon}^{\theta}-h_{\varepsilon}^{\theta}\right) \leq q_{C_{\varepsilon}}^{\mathrm{D}}\left(w_{\varepsilon}^{\theta}-h_{\varepsilon}^{\theta}\right)= \\
& \quad=\left\|\mathrm{d} w_{\varepsilon}^{\theta}-\mathrm{d} h_{\varepsilon}^{\theta}\right\|_{L_{2} T^{*} C_{\varepsilon}}^{2} \leq\left\|\mathrm{d} w_{\varepsilon}^{\theta}\right\|_{L_{2} T^{*} C_{\varepsilon}}^{2} \leq\left\|\mathrm{d} u_{\varepsilon}^{\theta}\right\|_{L_{2} T^{*} \widetilde{M}_{\varepsilon}}^{2}=q_{\widetilde{M}_{\varepsilon}}^{\theta}\left(u_{\varepsilon}^{\theta}\right) \leq c .
\end{aligned}
$$

where we have used the Min-max Principle in the first, $1 \leq 1 / \varepsilon^{2}$ in the second and Lemma 2.3.11 in the third inequality. It follows that

$$
\left\|h_{\varepsilon}^{\theta}\right\|_{L_{2}(C)} \leq\left\|w_{\varepsilon}^{\theta}-h_{\varepsilon}^{\theta}\right\|_{L_{2}(C)}+\left\|w_{\varepsilon}^{\theta}\right\|_{L_{2}(C)} \leq \sqrt{\frac{c}{\lambda_{1}^{\mathrm{D}}\left(C_{1}\right)}}+\sqrt{c} .
$$

In the same way we prove

$$
q_{C_{1}}\left(h_{\varepsilon}^{\theta}\right) \leq q_{C_{\varepsilon}}\left(h_{\varepsilon}^{\theta}\right)=\left\|\mathrm{d} h_{\varepsilon}^{\theta}\right\|_{L_{2} T^{*} C_{\varepsilon}}^{2} \leq\left\|\mathrm{d} w_{\varepsilon}^{\theta}\right\|_{L_{2} T^{*} C_{\varepsilon}}^{2} \leq\left\|\mathrm{d} u_{\varepsilon}^{\theta}\right\|_{L_{2} T^{*} \widetilde{M}_{\varepsilon}}^{2}=q_{\widetilde{M}_{\varepsilon}}^{\theta}\left(u_{\varepsilon}^{\theta}\right) \leq c .
$$

Again, we have used $1 \leq 1 / \varepsilon^{2}$ in the first and Lemma 2.3.11 in the second inequality. Since the $\mathscr{H}^{1}$-norm is equivalent to the quadratic form norm of the Laplacian there exists a constant $c^{\prime}>0$ such that

$$
\|h\|_{\mathscr{H}^{1}(C)}^{2} \leq c^{\prime}\left(\|h\|_{L_{2}(C)}^{2}+q_{C_{1}}(h)\right)
$$

for all $h \in \mathscr{H}^{1}(C)$. Therefore, the first part of the lemma follows.

Furthermore, the sequence $\left(h_{\varepsilon_{m}}^{\theta_{m}}\right)_{m}$ is bounded for any sequences $\left(\varepsilon_{m}\right)_{m}$ and $\left(\theta_{m}\right)_{m}$. Thus the weak sequential compactness of bounded subsets of the Hilbert space $\mathscr{H}^{1}(C)$ (see Theorem 1.1.1) implies the existence of a subsequence $\left(h_{n}\right)_{n}$ converging weakly to an element $h \in \mathscr{H}^{1}(C)$. The strong convergence is just a consequence of the Rellich-Kondrachov Theorem 2.2.4.

Note that the element $h$ in general depends on the sequences $\left(\varepsilon_{m}\right)_{m}$ and $\left(\theta_{m}\right)_{m}$. We will show in the next lemmas that this is not the case. First we prove that the limit $h$ is constant in spherical direction, secondly we prove that it is harmonic.

5.3.3. Lemma. The function $h: I \times \mathbb{S} \longrightarrow \mathbb{C}$ is independent of the second variable.

Proof. Let $P$ be the projection onto the space of functions in $L_{2}(C)=L_{2}(I) \otimes L_{2}(\mathbb{S})$ constant in the second variable. Note that this is a closed subspace. Furthermore, let $P^{\perp} w:=w-P w$ be the projection onto the orthogonal complement. The smallest eigenvalue of the quadratic form $q_{\mathbb{S}}(w):=\int_{C}\left|\mathrm{~d}_{\mathbb{S}} w\right|^{2}$ in $L_{2}(C)$ is 0 . The corresponding eigenspace is the range of the projection $P$. The first non-zero eigenvalue of $q_{\mathbb{S}}$ is $\lambda_{2}(\mathbb{S})>0$. Therefore

$$
\begin{aligned}
\lambda_{2}(\mathbb{S})\left\|P^{\perp} h_{\varepsilon}\right\|_{L_{2}(C)}^{2} \leq q_{\mathbb{S}}\left(P^{\perp} h_{\varepsilon}\right)=\int_{C}\left|\mathrm{~d}_{\mathbb{S}} h_{\varepsilon}-\mathrm{d}_{\mathbb{S}} P h_{\varepsilon}\right|^{2} \leq \\
\quad \leq \varepsilon^{2} \int_{C}\left(\left|\partial_{t} h_{\varepsilon}\right|^{2}+\frac{1}{\varepsilon^{2}}\left|\mathrm{~d}_{\mathbb{S}} h_{\varepsilon}\right|^{2}\right)=\varepsilon^{2} q_{C_{\varepsilon}}\left(h_{\varepsilon}\right)
\end{aligned}
$$

where we have used $\mathrm{d}_{\mathbb{S}} P h_{\varepsilon}=0$ since functions in the range of $P$ are constant in $\sigma \in \mathbb{S}$. By Lemma 5.3.2 we have $\left\|P^{\perp} h_{\varepsilon}\right\| \leq \varepsilon^{2} c / \lambda_{2}(\mathbb{S})$. Finally, $P^{\perp} h=0$ as $\varepsilon=\varepsilon_{n} \rightarrow 0$, and $P h=h$ since $h_{n} \rightarrow h$ strongly. 
In the sequel we sometimes do not distinguish between functions on $I$ and functions on $C$ constant in spherical direction.

5.3.4. Lemma. The function $h$ is harmonic on I, i.e., $\partial_{t t} h=0$.

Proof. Let $w \in C_{c}^{\infty}(C)$ be constant in the second variable (i.e., $w \in C_{c}^{\infty}(I)$ ). Then

$$
\left\langle-\partial_{t t} h, w\right\rangle=\left\langle\partial_{t} h, \partial_{t} w\right\rangle=\lim _{n \rightarrow \infty}\left\langle\partial_{t} h_{n}, \partial_{t} w\right\rangle=\lim _{n \rightarrow \infty} q_{C_{\varepsilon_{n}}}\left(h_{n}, w\right)=\lim _{n \rightarrow \infty}\left\langle\Delta_{C_{\varepsilon_{n}}} h_{n}, w\right\rangle=0
$$

due to Lemma 5.3.2 and 5.3.3. Thus $\partial_{t t} h$ is orthogonal to the space of functions constant in the second variable but also constant in the second variable by Lemma 5.3.3 so we have $\partial_{t t} h=0$.

5.3.5. Lemma. We have $h=0$.

Proof. It is sufficient to prove $h \uparrow_{\partial I}=0$ as a result of the maximum principle for harmonic functions. Here, we could argue even simplier: a harmonic function in one dimension is affine linear, therefore $h=0$ is equivalent to $h \uparrow_{\partial I}=0$.

Let $f \in C^{\infty}(I)$. By the Gauss-Green Formula (2.18) we have

$$
\begin{aligned}
{\left[\left\langle h, \partial_{t} f\right\rangle\right]_{\partial I}-\int_{I}\left\langle h, \partial_{t t} f\right\rangle=\int_{I}\left\langle\partial_{t} h, \partial_{t} f\right\rangle } & = \\
& =\lim _{n \rightarrow \infty} \int_{C}\left\langle\mathrm{~d} h_{n}, \mathrm{~d} f\right\rangle=\lim _{n \rightarrow \infty} \int_{\partial C}\left\langle h_{n}, \partial_{\mathrm{n}} f\right\rangle-\int_{I}\left\langle h, \partial_{t t} f\right\rangle
\end{aligned}
$$

due to the weak convergence of $\left(h_{n}\right)$ shown in Lemma 5.3.2. The $\theta$-periodicity implies the equality of the integrals over $\partial C$ and $Z_{\varepsilon}$. Together with Theorem 4.4.1 we obtain

$$
\int_{\partial C}\left|h_{\varepsilon}^{\theta}\right|^{2}=\int_{Z_{\varepsilon}}\left|u_{\varepsilon}^{\theta}\right|^{2} \leq \omega(\varepsilon) \int_{\widetilde{M}_{\varepsilon}}\left(\left|\mathrm{d} u_{\varepsilon}^{\theta}\right|^{2}+\left|u_{\varepsilon}^{\theta}\right|^{2}\right) \leq \omega(\varepsilon) c
$$

Therefore we have shown $\left[\left\langle h, \partial_{t} f\right\rangle\right]_{\partial I}=0$ for all $f \in C_{c}^{\infty}(I)$, i.e., $h=0$.

Proof of thereom 5.3.1. Let $\left(\varepsilon_{l}\right)_{l}$ be a sequence converging to 0. By Lemmas 5.3.2 to 5.3.5 every subsequence of $\left(h_{\varepsilon_{l}}^{\theta}\right)_{l}$ has a convergent sub-subsequence converging to 0 , i.e., $h_{\varepsilon_{l}}^{\theta} \rightarrow 0$ as $l \rightarrow \infty$ and every $\theta$.

Suppose this convergence is not uniform in $\theta$. Then we can find a number $\eta>0$ and sequences $\left(\varepsilon_{m}\right)_{m}$ and $\left(\theta_{m}\right)_{m}$ with $\varepsilon_{m} \rightarrow 0$ and $d\left(h_{\varepsilon_{m}}^{\theta_{m}}, 0\right) \geq \eta$ where $d$ denotes a metric defining the weak topology on a bounded subset of $\mathscr{H}^{1}(C)$. Lemma 5.3.2 implies the existence of a subsequence and a limit $\tilde{h}$ which must be different from 0. But Lemmas 5.3.3 to 5.3.5 imply $\tilde{h}=0$ which is a contradiction. 


\section{Conformal deformation}

In this chapter we do in some sense the converse of the work done by now. We start with a given periodic manifold $M^{\text {per }}$ of dimension $d \geq 2$ and deform the metric by a conformal factor $\rho_{\varepsilon}$ to obtain spectral gaps in the spectrum of the Laplacian. The idea is to let the conformal factor converge to the indicator function of a set $X$ with positive distance to the boundary of a period cell $M$ of $M^{\text {per }}$. This convergence is of course not uniform because of the discontinuity of the indicator function.

\subsection{Conformal deformation}

First, we state some facts about conformal deformations of a given metric $g$ of a $d$-dimensional Riemannian manifold $M$. Let $\rho_{\varepsilon}$ be a smooth, strictly positve function on $M$. Then we denote by $M_{\varepsilon}$ the manifold $M$ with metric $g_{\varepsilon}=\rho_{\varepsilon}^{2} g$.

We give some simple formulas how the conformal factor enters in the inner product and in the quadratic form of the Laplacian. The formulas in the case of differential forms will be needed in Section 7.4.

6.1.1. Lemma. The spaces $L_{2}\left(\mathbf{A}^{p} T M_{\varepsilon}\right)$ and $L_{2}\left(\mathbf{A}^{p} T M\right)$ are identical as vector spaces. Furthermore, for $u, v \in L_{2}\left(\mathbf{A}^{p} T M\right)$ we have

$$
\langle u, v\rangle_{L_{2}\left(\mathbf{A}^{p} T M_{\varepsilon}\right)}=\int_{M}\langle u(x), v(x)\rangle_{\mathbf{A}^{p} T_{x} M} \rho_{\varepsilon}^{d-2 p}(x) \mathrm{d} x .
$$

Denote by $\mathrm{d}^{*}$ and $\mathrm{d}_{\varepsilon}^{*}$ the formal adjoint of $\mathrm{d}: C_{c}^{\infty}\left(\mathbf{A}^{p-1} T M\right) \longrightarrow C_{c}^{\infty}\left(\mathbf{A}^{p} T M\right)$ with respect to the inner product of $L_{2}\left(\mathbf{A}^{\bullet} T M\right)$ and $L_{2}\left(\mathbf{A}^{\bullet} T M_{\varepsilon}\right)$. Then we have

$$
\mathrm{d}_{\varepsilon}^{*} u=\rho_{\varepsilon}^{2 p-d-2} \mathrm{~d}^{*}\left(\rho_{\varepsilon}^{d-2 p} u\right)
$$

for $u \in C_{c}^{\infty}\left(\mathbf{A}^{p} T M\right)$. Finally the quadratic form of the Laplacian on $\mathbf{A}^{p} T M_{\varepsilon}$ is given by

$$
q_{\mathbf{A}^{p} T M}(u)=\int_{M}\left(|d u|^{2} \rho_{\varepsilon}^{d-2 p}+\left|\mathrm{d}^{*}\left(\rho_{\varepsilon}^{d-2 p} u\right)\right|^{2} \rho_{\varepsilon}^{2 p-d}\right) \rho_{\varepsilon}^{-2}
$$

for $u \in \mathscr{H}^{1}\left(\mathbf{A}^{p} T M\right)$.

Proof. The volume form yields a factor $\rho_{\varepsilon}^{d}$. We further have $|u(x)|_{\mathbf{A}^{p} T M_{\varepsilon}}^{2}=\rho_{\varepsilon}^{-2 p}(x)|u(x)|_{\mathbf{A}^{p} T M}^{2}$ by (2.1). Note that the minus in the exponent occurs since we are dealing with the inverse matrix $\left(g_{\varepsilon}^{i j}\right)$ of $\left(g_{\varepsilon, i j}\right)$. From these remarks we easily conclude the three stated equations. 


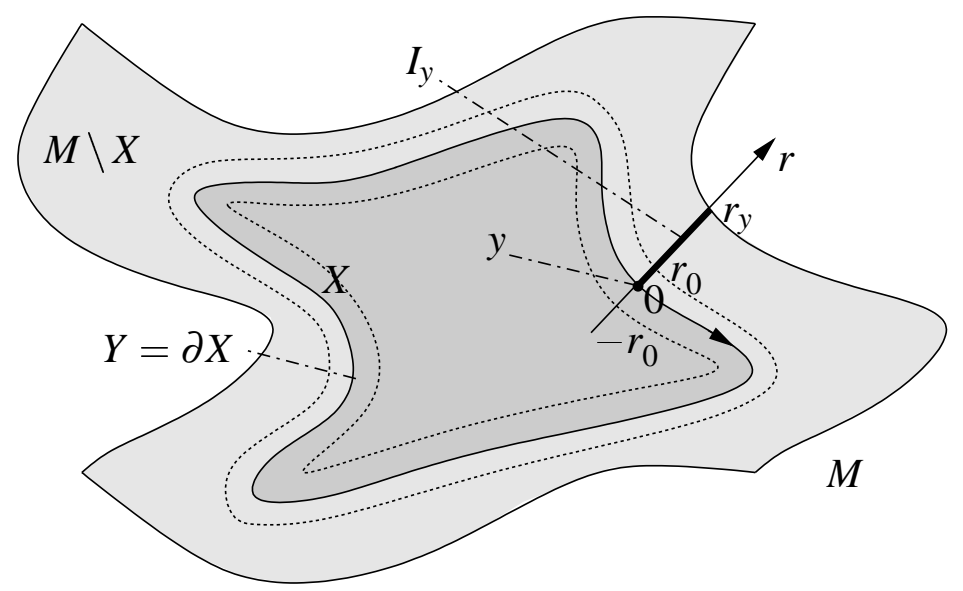

Figure 6.1: Normal coordinates $(r, y)$ parametrising a neighbourhood of $Y=\partial X$ and $M \backslash X$.

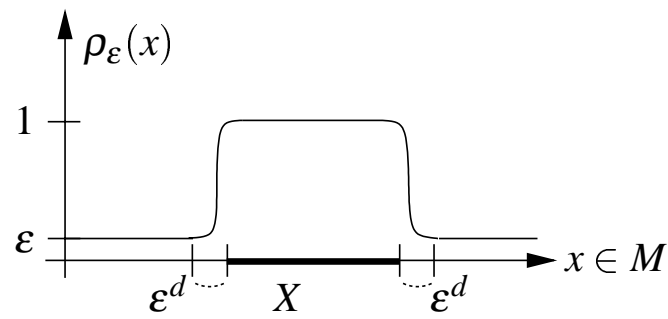

Figure 6.2: The conformal factor $\rho_{\varepsilon}$ leaving $X$ undeformed.

Now we deform a periodic manifold conformally. Let $M^{\text {per }}$ be a periodic Riemannian manifold with metric $g^{\text {per }}$ and cocompact abelian $\Gamma$-action (for details see Section 3.2). Let $X \subset M^{\text {per }}$ be a compact subset with smooth boundary such that $\gamma X \cap X \neq \emptyset$ implies $\gamma=1$. Then a period cell $M$ with $\operatorname{dist}(\partial X, \partial M)>0$ exists (see Figure 0.4 on page 6 or Figure 6.1).

We introduce normal or Fermi coordinates $(r, y)$ with respect to $Y:=\partial X$ (for details cf. [Cha93, Section 3.6]). Here, $r \in]-r_{0}, r_{0}[$ parametrises the normal direction and $y \in Y$ parametrises the tangential direction; $r<0$ corresponds to the interior of $X$ and $r=0$ corresponds to $Y$ (see Figure 6.1).

Furthermore, we assume that normal coordinates also exist on $\overline{M \backslash X}$, i.e., we suppose that $\overline{M \backslash X}$ can be parametrised by $(r, y)$ with $r \in I_{y}$ and $y \in Y$. Here, $I_{y}$ is a compact subset of $\mathbb{R}$ containing $\left[0, r_{0}\right]$ (see Figure 6.1). The existence of normal coordinates on $\overline{M \backslash X}$ is a geometrical restriction on $X$. For example, this condition is satisfied for a centered ball in a cube.

Suppose that, for each $\left.\left.\varepsilon>0, \rho_{\varepsilon}: M^{\text {per }} \longrightarrow\right] 0,1\right]$ is a smooth $\Gamma$-periodic function with the following properties (see Figure 6.2):

$$
\begin{array}{lr}
\rho_{\varepsilon}(x)=1 & \text { for all } x \in X, \\
\rho_{\varepsilon}(x)=\varepsilon & \text { for all } x \in M \text { with } \operatorname{dist}(x, X) \geq \varepsilon^{d} .
\end{array}
$$

Note that the function $\rho_{\varepsilon}$ converges pointwise to the indicator function of the set $X$. We define

$$
g_{\varepsilon}^{\text {per }}:=\rho_{\varepsilon}^{2} g^{\text {per }} \quad \text { resp. } \quad g_{\varepsilon}:=g^{\text {per }} \uparrow_{M}
$$


and call the resulting Riemannian manifolds $M_{\varepsilon}^{\text {per }}$ resp. $M_{\mathcal{\varepsilon}}$. We therefore obtain a conformally deformed $\Gamma$-periodic manifold $M_{\varepsilon}^{\text {per }}$ with periodic metric $g_{\varepsilon}^{\text {per }}$ and periodic Laplace operator $\Delta_{M_{\varepsilon}^{\text {per }}}$. The spectrum of this operator will be analysed by applying Floquet theory. We only have to analyse the behaviour of the $\theta$-periodic eigenvalues $\lambda_{k}^{\theta}\left(M_{\varepsilon}\right)$ of the corresponding Laplacian on $M_{\varepsilon}$.

In the rest of this chapter we show that the Dirichlet and Neumann eigenvalues on $M_{\varepsilon}$ (denoted by $\lambda_{k}^{\mathrm{D} / \mathrm{N}}\left(M_{\varepsilon}\right)$ ) converge to the eigenvalues of the Neumann boundary problem on $X$, provided the dimension $d$ of the manifold $M^{\text {per }}$ is greater than 2. The two dimensional case will be treated later.

6.1.2. Theorem. Suppose $M^{\text {per }}$ is of dimension $d \geq 3$. Suppose further that $X$ is a subset of a periodic cell $M$ with smooth boundary $\partial X$ and with $\operatorname{dist}(\partial X, \partial M)>0$ such that normal coordinates on $\overline{M \backslash X}$ exist (see Figure 6.1). We assume that $\left.\left.\left(\rho_{\varepsilon}: M \longrightarrow\right] 0,1\right]\right)_{\varepsilon}$ is a family of conformal factors satisfying Conditions (6.4) and (6.5). Then

$$
\lim _{\varepsilon \rightarrow 0} \lambda_{k}^{\mathrm{D} / \mathrm{N}}\left(M_{\varepsilon}\right)=\lambda_{k}^{\mathrm{N}}(X)
$$

where $M_{\varepsilon}$ denotes the conformally deformed period cell with metric given by (6.6). In particular, the $k$-th $\theta$-periodic eigenvalue $\lambda_{k}^{\theta}\left(M_{\varepsilon}\right)$ on $M_{\varepsilon}$ converges to $\lambda_{k}^{\mathrm{N}}(X)$.

Again, applying Floquet theory we obtain:

6.1.3. Corollary. For $\varepsilon$ small enough the $k$-th band $B_{k}\left(\Delta_{M_{\varepsilon}^{\text {per }}}\right)$ and the $(k+1)$-st band $B_{k+1}\left(\Delta_{M_{\varepsilon}^{\text {per }}}\right)$ do not overlap, provided that $\lambda_{k}(X)<\lambda_{k+1}(X)$. In particular, for given $N \in \mathbb{N}$ there exists $\varepsilon>0$ such that the periodic operator $\Delta_{M_{\varepsilon}^{\text {per }}}$ on the conformally deformed manifold $M$ has at least $N$ gaps.

We split the proof of the theorem in two parts: a lower bound for $\lambda_{k}^{\mathrm{N}}\left(M_{\varepsilon}\right)$ and an upper bound for $\lambda_{k}^{\mathrm{D}}\left(M_{\varepsilon}\right)$.

\subsection{Lower bounds for the eigenvalues}

We first prove lower bounds for the Neumann eigenvalues $\lambda_{k}^{\mathrm{N}}\left(M_{\varepsilon}\right)$. We need the following estimate on $M_{\mathcal{\varepsilon}} \backslash X$. Note that the next theorem is also true in dimension $d=2$. The idea is motivated by articles of C. Anné (cf. [Ann87], [Ann94, Lemme A] and [Ann99]).

6.2.1. Theorem. Suppose that normal coordinates exist on $\overline{M \backslash X}$. Let $\rho_{\varepsilon}(x)=\rho_{\varepsilon}(r)$ be a function satisfying conditions (6.4) and (6.5), i.e., $\rho_{\varepsilon}(r)=1$ for all $r<0$ and $\rho_{\varepsilon}(r)=\varepsilon$ for all $r \geq \varepsilon^{d}$.

Then we can find a function $\omega(\varepsilon) \rightarrow 0$ as $\varepsilon \rightarrow 0$ such that

$$
\int_{M_{\varepsilon} \backslash X}\left|u_{\varepsilon}\right|^{2} \leq \omega(\varepsilon)\left(\int_{M_{\varepsilon}}\left|u_{\varepsilon}\right|^{2}+\int_{M_{\varepsilon}}\left|\mathrm{d} u_{\varepsilon}\right|^{2}\right)
$$

for all $u_{\varepsilon} \in \mathscr{H}^{1}\left(M_{\varepsilon}\right)$. 
Proof. We proceed in the same way as in the proof of Theorem 4.4.1. We introduce normal coordinates as in Figure 6.1. For notational simplicity only, we assume that $I_{y}=\left[0, r_{y}\right]$ for some number $r_{0} \leq r_{y}$. Suppose that $u_{\varepsilon} \in C^{\infty}\left(M_{\varepsilon}\right)$ with $u_{\varepsilon}(r, y)=0$ for all $y \in Y$ and $r \leq-r_{0}$. As in (4.1) we have the orthogonal splitting

$$
g_{\varepsilon}=\rho_{\varepsilon}^{2}\left(\mathrm{~d} r^{2}+h_{(r, y)}\right)
$$

in normal coordinates where $h_{(r, \cdot)}$ is a parameter-dependent metric on $Y$. From (6.1) and (6.3) we obtain

$$
\int_{M_{\varepsilon}}\left|u_{\varepsilon}\right|^{2}=\int_{M}\left|u_{\varepsilon}\right|^{2} \rho_{\varepsilon}^{d} \quad \text { and } \quad \int_{M_{\varepsilon}}\left|\mathrm{d} u_{\varepsilon}\right|_{T^{*} M_{\varepsilon}}^{2}=\int_{M}\left|u_{\varepsilon}\right|_{T^{*} M}^{2} \rho_{\varepsilon}^{d-2} .
$$

By the Cauchy-Schwarz Inequality we have

$$
\begin{aligned}
\left|u_{\varepsilon}(s, y)\right|^{2} & =\left|\int_{-r_{0}}^{s} \partial_{r} u_{\varepsilon}(r, y) \mathrm{d} r\right|^{2} \\
& \leq \int_{-r_{0}}^{s}(\operatorname{det} g(r, y))^{-\frac{1}{2}} \mathrm{~d} r \cdot \int_{-r_{0}}^{s}\left(\left|\partial_{r} u_{\varepsilon}\right|^{2}(\operatorname{det} g)^{\frac{1}{2}}\right)(r, y) \mathrm{d} r
\end{aligned}
$$

for $0 \leq s \leq r_{y}$. Since $Y$ is compact we can estimate the first integral by $c>0$. Therefore integrating over $s \in I_{y}$ and $y \in Y$ yields

$$
\begin{aligned}
\int_{M_{\varepsilon} \backslash X}\left|u_{\varepsilon}\right|^{2} & =\int_{y \in Y} \int_{s=0}^{r_{y}}\left(\left|u_{\varepsilon}\right|^{2}(\operatorname{det} g)^{\frac{1}{2}} \rho_{\varepsilon}^{d}\right)(s, y) \mathrm{d} s \mathrm{~d} y \\
& \leq c \int_{y \in Y} \int_{s=0}^{r_{y}}(\operatorname{det} g)^{\frac{1}{2}}(s, y) \rho_{\varepsilon}^{d}(s) \int_{r=-r_{0}}^{s}\left(\left|\partial_{r} u_{\varepsilon}\right|^{2}(\operatorname{det} g)^{\frac{1}{2}}\right)(r, y) \mathrm{d} r \mathrm{~d} s \mathrm{~d} y .
\end{aligned}
$$

We can estimate the $s$-dependent terms as follows: for $0 \leq s \leq \varepsilon^{d}$ we have $\rho_{\varepsilon}(s)=\varepsilon$ by Assumption (6.5). Furthermore, there exists a constant $c^{\prime}>0$ such that $(\operatorname{det} g)^{\frac{1}{2}}(s, y) \leq c^{\prime}$ for all $y \in Y$ and $0 \leq s \leq r_{y}$ since $\overline{M \backslash X}$ is compact. Therefore the integral over $0 \leq s \leq \varepsilon^{d}$ and $\varepsilon^{d} \leq s \leq r_{y}$ can be estimated by $c^{\prime} \varepsilon^{d}$. We conclude

$$
\begin{aligned}
\int_{M_{\varepsilon} \backslash X}\left|u_{\varepsilon}\right|^{2} & \leq c c^{\prime} \varepsilon^{d} \int_{y \in Y} \int_{r=-r_{0}}^{r_{y}}\left(\left|\partial_{r} u_{\varepsilon}\right|^{2}(\operatorname{det} g)^{\frac{1}{2}}\right)(r, y) \mathrm{d} r \mathrm{~d} y \\
& \leq c c^{\prime} \varepsilon^{2} \int_{y \in Y} \int_{r=-r_{0}}^{r_{y}}\left(\left|\partial_{r} u_{\varepsilon}\right|^{2}(\operatorname{det} g)^{\frac{1}{2}} \rho_{\varepsilon}^{d-2}\right)(r, y) \mathrm{d} r \mathrm{~d} y \\
& \leq c c^{\prime} \varepsilon^{2} \int_{M_{\varepsilon}}\left|\mathrm{d} u_{\varepsilon}\right|^{2}
\end{aligned}
$$

where we have used $\rho_{\varepsilon} \geq \varepsilon$ in the second line.

If $u_{\varepsilon}(r, y) \neq 0$ for some $y \in Y$ and $r<-r_{0}$ we multiply $u_{\varepsilon}$ with a cut-off function $\chi$ such that $\chi(r)=1$ for $r \geq-r_{0} / 2$ and $\chi(r)=0$ for $r \leq-r_{0}$. Note that supp $\chi \subset X$, i.e., on supp $\chi$, there is no conformal deformation. If $u_{\varepsilon} \in \mathscr{H}^{1}\left(M_{\varepsilon}\right)$ we apply an approximation argument.

We emphasize that the function $\omega(\varepsilon)$ only depends on the geometry of $X$ and $M$. Again we apply the Main Lemma to prove a lower bound on the Neumann eigenvalue $\lambda_{k}^{\mathrm{N}}\left(M_{\varepsilon}\right)$, this time with

$$
\begin{array}{rlrl}
\mathscr{H}_{\varepsilon} & :=L_{2}\left(M_{\varepsilon}\right), & \operatorname{dom} q_{\varepsilon}:=\mathscr{H}^{1}\left(M_{\varepsilon}\right), & q_{\varepsilon}:=q_{M_{\varepsilon}}^{\mathrm{N}} \\
\mathscr{H}^{\prime}:=L_{2}(X), & \operatorname{dom} q^{\prime}:=\mathscr{H}^{1}(X), & q^{\prime}:=q_{X}^{\mathrm{N}}
\end{array}
$$


Here the operator $\Phi_{\varepsilon}$ is just the restriction of $u_{\varepsilon}$ to $X$, i.e., $\Phi_{\varepsilon} u_{\varepsilon}:=\left.u_{\varepsilon}\right|_{X}$. We verify the conditions of Corollary 1.4.4. Condition 1 resp. (1.15) is satisfied by Theorem 6.2.1. Condition 2 resp. (1.16) is trivially satisfied because of

$$
q_{\varepsilon}\left(u_{\varepsilon}\right)=\int_{M_{\varepsilon}}\left|\mathrm{d} u_{\varepsilon}\right|^{2} \geq \int_{X}\left|\mathrm{~d} u_{\varepsilon}\right|^{2}=q^{\prime}\left(\Phi_{\varepsilon} u_{\varepsilon}\right) .
$$

Condition 3 is satisfied by the embedding $\mathscr{H}^{1}(X) \subset \mathscr{H}_{\theta}^{1}\left(M_{\varepsilon}\right)$ which implies $\lambda_{k}^{\mathrm{N}}\left(M_{\varepsilon}\right) \leq$ $\lambda_{k}^{\mathrm{D}}(X):=c_{k}$ by the Min-max Principle. Therefore Theorem 1.4.2 yields

$$
\lambda_{k}^{\mathrm{N}}(X)-\underline{\delta}_{k}(\varepsilon) \leq \lambda_{k}^{\mathrm{N}}\left(M_{\varepsilon}\right)
$$

with $\underline{\delta}_{k}(\varepsilon) \rightarrow 0$ as $\varepsilon \rightarrow 0$.

\subsection{Upper bounds for the eigenvalues}

Here we use our assumption that the dimension satisfies $d \geq 3$. We apply our Main Lemma with

$$
\begin{aligned}
& \mathscr{H}:=L_{2}(X), \\
& \operatorname{dom} q:=\mathscr{H}^{1}(X), \\
& q:=q_{X}^{\mathrm{N}} \\
& \mathscr{H}_{\varepsilon}^{\prime}:=L_{2}\left(M_{\varepsilon}\right) \text {, } \\
& \operatorname{dom} q_{\varepsilon}^{\prime}:=\mathscr{H}^{1}\left(M_{\varepsilon}\right) \text {, } \\
& q_{\varepsilon}^{\prime}:=q_{M_{\varepsilon}}^{\mathrm{D}} \text {. }
\end{aligned}
$$

Let $\Phi u$ be an extension of $u$ which lies in $\mathscr{H}^{1}\left(M_{\varepsilon}\right)$.

Again we have to verify the conditions of Theorem 1.4.2. Condition 1 is satisfied because of

$$
\|u\|^{2}=\int_{X}|u|^{2} \leq \int_{M_{\varepsilon}}|\Phi u|^{2}=\|\Phi u\|^{2}
$$

In the case $d \geq 3$ Condition 2 is satisfied because of

$$
\left|q_{\varepsilon}^{\prime}(\Phi u)-q(u)\right|=\int_{M_{\varepsilon} \backslash X}|\mathrm{~d} \Phi u|_{T^{*} M_{\varepsilon}}^{2}=\int_{M \backslash X}|\mathrm{~d} \Phi u|_{T^{*} M}^{2} \rho_{\varepsilon}^{d-2} \rightarrow 0
$$

by the Lebesgue convergence theorem and the requirement (6.5) which implies that $\rho_{\varepsilon}(x) \rightarrow 0$ for all $x \in M \backslash X$. Note that the convergence depends on $u$ (see Remark 1.4.3). Condition 3 is not necessary because $\mathscr{H}$ is independent of $\varepsilon$. Theorem 1.4 .2 yields

$$
\lambda_{k}^{\mathrm{D}}\left(M_{\varepsilon}\right) \leq \lambda_{k}^{\mathrm{N}}(X)+\bar{\delta}_{k}(\varepsilon)
$$

with $\bar{\delta}_{k}(\varepsilon) \rightarrow 0$ as $\varepsilon \rightarrow 0$. From (6.9), (6.11) and the Dirichlet-Neumann enclosure (3.12) we obtain

$$
\lambda_{k}^{\mathrm{N}}(X)-\underline{\delta}_{k}(\varepsilon) \leq \lambda_{k}^{\mathrm{N}}\left(M_{\varepsilon}\right) \leq \lambda_{k}^{\theta}\left(M_{\varepsilon}\right) \leq \lambda_{k}^{\mathrm{D}}\left(M_{\varepsilon}\right) \leq \lambda_{k}^{\mathrm{N}}(X)+\bar{\delta}_{k}(\varepsilon) .
$$

We therefore have proven Theorem 6.1.2. 


\section{The two-dimensional case}

In dimension 2, the special form of the Raleigh quotient of the Laplacian on the conformally perturbed manifold $M_{\varepsilon}^{\text {per }}$ causes a different behaviour. The $\theta$-periodic eigenvalues of the Laplacian on a period cell $M_{\varepsilon}$ still converge but the limit depends on $\theta$. Since the corresponding limit operator is quite complicated, we are only able to construct a simple example of a conformally perturbed 2-dimensional manifold with an arbitrary number of gaps in the spectrum of its Laplacian.

\subsection{What is different in the two-dimensional case?}

In the preceding chapter we have shown that the $k$-th band $B_{k}\left(M_{\varepsilon}^{\text {per }}\right)$ of $\Delta_{M_{\varepsilon}^{\text {per }}}$ converges to the $k$-th Neumann eigenvalue $\lambda_{k}^{\mathrm{N}}(X)$ provided that $X$ satisfies certain geometrical conditions, that the conformal factors $\rho_{\varepsilon}(\varepsilon>0)$ satisfy Conditons (6.4) and (6.5), and that the dimension is at least 3 . The following lemma shows that in dimension 2 , the behaviour is completely different:

7.1.1. Lemma. Suppose that $M^{\text {per }}$ is connected. Suppose further that the conformal factors $\rho_{\varepsilon}$ satisfy Conditons (6.4) and (6.5). Suppose in addition that $\rho_{\varepsilon}$ are monotonically decreasing if $\varepsilon \searrow 0$. Then we always have the inclusion

$$
B_{1}\left(M_{\varepsilon_{0}}^{\text {per }}\right) \subset B_{1}\left(M_{\mathcal{\varepsilon}}^{\text {per }}\right), \quad 0<\varepsilon \leq \varepsilon_{0},
$$

for the first band of the periodic Laplacian $\Delta_{M_{\varepsilon_{0}}^{\text {per }}}$, where $B_{1}\left(M_{\varepsilon_{0}}^{\text {per }}\right)$ has non-empty interior.

In particular, the first band cannot collapse to the point 0 , the first Neumann eigenvalue, as in the higher dimensional case.

Proof. As a result of the Min-max Principle (Theorem 1.3.3), the first eigenvalue is given by

$$
\lambda_{1}^{\theta}\left(M_{\varepsilon}\right)=\inf _{u \in \mathscr{H}_{\theta}^{1}\left(M_{\varepsilon}\right)} \frac{\int_{M}|\mathrm{~d} u|^{2}}{\int_{M}|u|^{2} \rho_{\varepsilon}^{2}},
$$

and therefore monotonically increasing as $\varepsilon \searrow 0$. Note that $\mathscr{H}_{\theta}^{1}\left(M_{\varepsilon}\right)$ is independent of $\varepsilon$ as vector space. We further have $\lambda_{1}^{\theta=1}\left(M_{\varepsilon}\right)=0$ since in the periodic case the constant function is an eigenfunction. By the continuous dependence of the eigenvalues on $\theta$ (see Theorem 3.4.3) we have $\left[0, \lambda_{1}^{\theta_{0}}\left(M_{\varepsilon_{0}}\right)\right]=B_{1}\left(M_{\varepsilon_{0}}^{\text {per }}\right)$ for some $\theta_{0}$. Therefore

$$
B_{1}\left(M_{\varepsilon_{0}}^{\text {per }}\right)=\left[0, \lambda_{1}^{\theta_{0}}\left(M_{\varepsilon_{0}}\right)\right] \subset\left[0, \lambda_{1}^{\theta_{0}}\left(M_{\varepsilon}\right)\right] \subset B_{1}\left(M_{\varepsilon}^{\text {per }}\right) .
$$

By Lemma 3.4.5, $B_{1}\left(M_{\varepsilon_{0}}^{\text {per }}\right)$ has non-empty interior. 
In arbitrary dimension the Raleigh quotient is of the form

$$
\lambda_{1}^{\theta}\left(M_{\varepsilon}\right)=\inf _{u \in \mathscr{H}_{\theta}^{1}\left(M_{\varepsilon}\right)} \frac{\int_{M}|\mathrm{~d} u|^{2} \rho_{\varepsilon}^{d-2}}{\int_{M}|u|^{2} \rho_{\varepsilon}^{d}} .
$$

As before, the space $\mathscr{H}_{\theta}^{1}\left(M_{\mathcal{\varepsilon}}\right)$ is independent of $\varepsilon$ as vector space which we denote by $\mathscr{H}_{\theta}^{1}(M)$. But for dimension $d \geq 3$ this quotient can be minimized by functions constant on $X$. These functions can still be $\theta$-periodic since their behaviour on $M_{\mathcal{\varepsilon}} \backslash X$ is not important because of the smallness of $\rho_{\varepsilon}$ there.

\subsection{Limit form in two dimensions}

To motivate which is the right candidate for the limit quadratic form in two dimensions, we use the special structure here. The quadratic form does not depend any more on $\varepsilon$, only the norm does. By interchanging their rôles we can prove a monotonicity result for the eigenvalues and specify the limit operator. The abstract background is given in Section 1.5.

We apply Lemma 1.5 .1 to the nonperiodic case since then we have $0 \notin \operatorname{spec} \Delta_{M_{\varepsilon}}^{\theta}$ : any eigenfunction with eigenvalue 0 would have to be constant but the nonperiodicity forces the constant to be 0 . In particular, we set

$$
\mathscr{H}_{\varepsilon}=L_{2}\left(M_{\varepsilon}\right), \quad Q_{\varepsilon}=\Delta_{M_{\varepsilon}}^{\theta} \quad \text { and } \quad q(u)=\int_{M}|\mathrm{~d} u|^{2}=\|u\|_{\mathscr{\mathscr { H }}^{\circ}}^{2}
$$

With the notation of Section 1.5 we obtain

$$
\widetilde{\mathscr{H}}=\operatorname{dom} q=\mathscr{H}_{\theta}^{1}(M), \quad \lambda_{k}(\varepsilon)=\lambda_{k}^{\theta}\left(M_{\varepsilon}\right) \quad \text { and } \quad \widetilde{q}_{\varepsilon}(u)=\int_{M}|u|^{2} \rho_{\varepsilon}^{2}=\|u\|_{\mathscr{H}_{\varepsilon}}^{2} .
$$

We suppose the conformal factor $\rho_{\varepsilon}$ to be monotone. Therefore

$$
\widetilde{q}_{\varepsilon}(u)=\int_{M}|u|^{2} \rho_{\varepsilon}^{2} \searrow \int_{X}|u|^{2}=: \widetilde{q}_{0}(u) .
$$

The limit form $\widetilde{q}_{0}$ corresponds to an operator $\widetilde{Q}_{0}$ wich is also compact (by the monotonicity and the Min-max Principle for example). Since the eigenvalues of $\widetilde{Q}_{\varepsilon}$, the operator corresponding to the quadratic form $\widetilde{q}_{\varepsilon}$, are exactly the reciprocals of $\lambda_{k}^{\theta}\left(M_{\varepsilon}\right)$ by Lemma 1.5 .1 , all we have to do is to analyse the eigenvalues $\widetilde{\lambda}_{k}$ of $\widetilde{Q}_{0}$ (denoted in decreasing order $\widetilde{\lambda}_{1} \geq \widetilde{\lambda}_{2} \geq \cdots \geq \tilde{\lambda}_{k} \geq 0$ ). The eigenvalues $\lambda_{k}^{\theta}\left(M_{\varepsilon}\right)$ are dominated by the Dirichlet eigenvalues $\lambda_{k}^{\mathrm{D}}(X)$, therefore we have the minoration

$$
\tilde{\lambda}_{k}=1 / \lambda_{k}^{\theta}\left(M_{\varepsilon}\right) \geq 1 / \lambda_{k}^{\mathrm{D}}(X)>0 \text {. }
$$

Therefore we are only interested in strictly positive eigenvalues $\tilde{\lambda}_{k}>0$. We compute the orthogonal complement (in $\widetilde{\mathscr{H}}$ ) of

$$
\operatorname{ker} \widetilde{Q}_{0}=\left\{u \in \mathscr{H}_{\theta}^{1}(M) \mid u \uparrow_{X}=0\right\}=: \stackrel{\mathscr{H}}{\theta}_{\theta}^{1}(M \backslash X) .
$$


In Lemma 3.5.2 we have shown that

$$
(\operatorname{ker} \widetilde{Q})^{\perp}=\left\{h \in \mathscr{H}_{\theta}^{1}(M) \mid \Delta h \uparrow_{M \backslash X}=0, \mathrm{~d} h \text { is } \theta \text {-periodic on } M \backslash X\right\} .
$$

Since we do not really want to analyse the limit operator $\left.\widetilde{Q}_{0}\right\rceil_{\mathscr{H}_{0}}$ in the inversed situation we try to bring it back in the usual context, i.e., we try to construct a quadratic form $q_{0}^{\theta}$ in the Hilbert space $L_{2}(X)$. In Theorem 3.5.3 we have shown that every $u \in \mathscr{H}^{1}(X)$ can be extended in a unique way to an element $h=\Phi u \in \widetilde{\mathscr{H}_{0}}$ (even in the periodic case).

Therefore we can define the limit quadratic form in the Hilbert space $L_{2}(X)$. For $u \in$ $\operatorname{dom} q_{0}^{\theta}:=\mathscr{H}^{1}(X)$ we set

$$
q_{0}^{\theta}(u):=\int_{M}|\mathrm{~d} \Phi u|^{2},
$$

i.e., we extend $u$ harmonically onto $M \backslash X$. Since we have

$$
q_{X}^{\mathrm{N}} \leq q_{0}^{\theta} \leq q_{X}^{\mathrm{D}}
$$

in the sense of quadratic forms (see Definition 1.3.4), $q_{0}^{\theta}$ has also purely discrete spectrum denoted by $\lambda_{k}\left(q_{0}^{\theta}\right)$ satisfying

$$
\lambda_{k}^{\mathrm{N}}(X) \leq \lambda_{k}\left(q_{0}^{\theta}\right) \leq \lambda_{k}^{\mathrm{D}}(X)
$$

7.2.1. Theorem. Suppose $M^{\text {per }}$ is of dimension $d=2$. Suppose further that $X$ is a subset of a periodic cell $M$ with smooth boundary $\partial X$ and with $\operatorname{dist}(\partial X, \partial M)>0$ such that normal coordinates on $\overline{M \backslash X}$ exist (see Figure 6.1). We assume that $\left.\left.\left(\rho_{\varepsilon}: M \longrightarrow\right] 0,1\right]\right)_{\varepsilon}$ is a family of conformal factors satisfying conditions (6.4), (6.5) and which is, in addition, monotonically decreasing as $\varepsilon \searrow 0$. Then

$$
\lim _{\varepsilon \rightarrow 0} \lambda_{k}^{\theta}\left(M_{\varepsilon}\right)=\lambda_{k}\left(q_{0}^{\theta}\right)
$$

uniformly in $\theta \in \hat{\Gamma}$ where $M_{\varepsilon}$ denotes the conformally deformed period cell $M$ with metric given by (6.6).

Proof. Again we apply our Main Lemma (Theorem 1.4.2) with

$$
\begin{aligned}
& \mathscr{H}_{\varepsilon}:=L_{2}\left(M_{\varepsilon}\right), \\
& \operatorname{dom} q_{\varepsilon}:=\mathscr{H}_{\theta}^{1}\left(M_{\varepsilon}\right), \\
& q_{\varepsilon}:=q_{M_{\varepsilon}}^{\theta} \\
& \mathscr{H}^{\prime}:=L_{2}(X) \text {, } \\
& \operatorname{dom} q^{\prime}:=\mathscr{H}^{1}(X), \\
& q^{\prime}:=q_{0}^{\theta}
\end{aligned}
$$

where $\Phi_{\varepsilon}:=\left.u_{\varepsilon}\right|_{X}$. Condition 1 is satisfied because

$$
\left|\left\|\Phi_{\varepsilon} u_{\varepsilon}\right\|^{2}-\left\|u_{\varepsilon}\right\|^{2}\right|=\int_{M_{\varepsilon} \backslash X}\left|u_{\varepsilon}\right|^{2} \leq \omega(\varepsilon)\left(\int_{M_{\varepsilon}}\left|u_{\varepsilon}\right|^{2}+\int_{M_{\varepsilon}}\left|\mathrm{d} u_{\varepsilon}\right|^{2}\right)
$$

converges to 0 for every $q_{M_{\varepsilon}}^{\theta}$-bounded family $\left(u_{\varepsilon}\right)_{\varepsilon}$ by Theorem 6.2.1. Since $\omega$ does not depend on $\theta$ this convergence is even uniform in $\theta$. Condition 2 is satisfied as a result of Lemma 2.3.11 which says that harmonic functions minimize the energy integral:

$$
\begin{aligned}
q_{\varepsilon}\left(u_{\varepsilon}\right)=\int_{M_{\varepsilon}}\left|\mathrm{d} u_{\varepsilon}\right|^{2} & =\int_{X}\left|\mathrm{~d} u_{\varepsilon}\right|^{2}+\int_{M \backslash X}\left|\mathrm{~d} u_{\varepsilon}\right|^{2} \\
& \geq \int_{X}\left|\mathrm{~d} u_{\varepsilon}\right|^{2}+\int_{M \backslash X}\left|\mathrm{~d} h_{\varepsilon}\right|^{2}=q_{0}^{\theta}\left(\Phi_{\varepsilon} u_{\varepsilon}\right) .
\end{aligned}
$$


Here, $h_{\varepsilon}$ is the harmonic extension of $u_{\varepsilon}$ restricted to $M \backslash X$. It is important to note that this estimate only works in dimension 2 since then there is no $\rho_{\varepsilon}$-factor in the energy integral. As usual, condition 3 is satisfied by the embedding $\mathscr{H}^{1}(X) \subset \mathscr{H}_{\theta}^{1}\left(M_{\varepsilon}\right)$ which implies $\lambda_{k}^{\theta}\left(M_{\varepsilon}\right) \leq$ $\lambda_{k}^{\mathrm{D}}(X):=c_{k}$ via the Min-max Principle. Therefore, the Main Lemma yields

$$
\lambda_{k}\left(q_{0}^{\theta}\right)-\delta_{k}(\varepsilon) \leq \lambda_{k}^{\theta}\left(M_{\varepsilon}\right)
$$

with $\delta_{k}(\varepsilon) \rightarrow 0$ as $\varepsilon \rightarrow 0$ uniformly in $\theta$.

For the other inequality we apply the Main Lemma with

$$
\begin{aligned}
& \mathscr{H}:=L_{2}(X), \\
& \operatorname{dom} q:=\mathscr{H}^{1}(X), \\
& q:=q_{0}^{\theta} \\
& \mathscr{H}_{\varepsilon}^{\prime}:=L_{2}\left(M_{\varepsilon}\right) \text {, } \\
& \operatorname{dom} q_{\varepsilon}^{\prime}:=\mathscr{H}_{\theta}^{1}\left(M_{\varepsilon}\right) \text {, } \\
& q_{\varepsilon}^{\prime}:=q_{M_{\varepsilon}}^{\theta}
\end{aligned}
$$

where $\Phi u$ is the harmonic extension as in Theorem 3.5.3. Again we have to verify the conditions of Theorem 1.4.2. Condition 1 is satisfied because of

$$
\|u\|^{2}=\int_{X}|u|^{2} \leq \int_{M_{\varepsilon}}|\Phi u|^{2}=\|\Phi u\|^{2} .
$$

Condition 2 is trivially satisfied because of

$$
q_{0}^{\theta}(u)=\int_{X}|\mathrm{~d} u|^{2}+\int_{M \backslash X}|\mathrm{~d} \Phi u|^{2}=\int_{M}|\mathrm{~d} \Phi u|^{2}=q_{\varepsilon}^{\prime}(\Phi u) .
$$

Condition 3 is not necessary because $\mathscr{H}$ is independent of $\varepsilon$. Therefore, by Theorem 1.4 .2 we obtain

$$
\lambda_{k}^{\theta}\left(M_{\varepsilon}\right) \leq \lambda_{k}\left(q_{0}^{\theta}\right)
$$

Note that we do not have an error term since in both conditions we have used the inequalities in Theorem 1.4.2.

7.2.2. Remark. In the same way we can show the convergence $\lambda_{k}^{\mathrm{D} / \mathrm{N}}\left(M_{\varepsilon}\right) \rightarrow \lambda_{k}^{\mathrm{D} / \mathrm{N}}(0)$ where the limit form $q_{0}^{\mathrm{D} / \mathrm{N}}$ is defined similarly (here we use the harmonic extension with Dirichlet resp. Neumann boundary conditions on $\partial M$ ).

We want to prove the existence of gaps for $\varepsilon$ small enough at least in the special situation given in the next section. By Theorem 7.2.1 we only need to calculate the $\theta$-dependent eigenvalues $\lambda_{k}^{\theta}(0)$ of the limit operator $Q_{0}^{\theta}$. If we can find bounds $0<\bar{\lambda}_{k}<\underline{\lambda}_{k+1}$ independent of $\theta$ with

$$
\lambda_{k}^{\theta}(0) \leq \bar{\lambda}_{k}<\underline{\lambda}_{k+1} \leq \lambda_{k+1}^{\theta}(0)
$$

for all $\theta \in \hat{\Gamma}$, we have established the existence of a gap between the $k$-th and the $(k+1)$-st band.

For the concrete example in the next section we need some information about the domain of the operator corresponding to the quadratic form $q_{0}^{\theta}$ via (1.4). Again, $\Phi^{\theta} u$ denotes the ( $\theta$ periodic) harmonic extension of $u$ onto $M \backslash X$. Furthermore, $\partial_{\mathrm{n}}$ denotes the normal derivative on $\partial X$. 


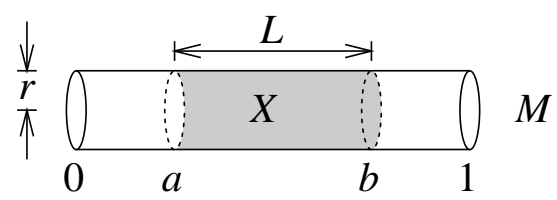

Figure 7.1: The period cell $M$ of the cylinder $M^{\text {per }}=\mathbb{R} \times \mathbb{S}^{1}$.

7.2.3. Lemma. The domain of the operator $Q_{0}^{\theta}$ corresponding to the limit quadratic form $q_{0}^{\theta}$ is given by

$$
\operatorname{dom} Q_{0}^{\theta}=\left\{u \in \mathscr{H}^{2}(X) \mid \partial_{\mathrm{n}} u=\partial_{\mathrm{n}} \Phi^{\theta} u \quad \text { on } \partial X\right\}
$$

Furthermore, $Q_{0}^{\theta} u=\Delta_{X} u$ for $u \in \operatorname{dom} Q_{0}^{\theta}$.

Proof. The lemma follows from the Gauss-Green formula (2.18) and Theorem 1.2.2. Note that the integral over $\partial M$ vanishes by Lemma 3.5.1. Furthermore, $u, \Delta u \in L_{2}(X)$ imply $u \in \mathscr{H}^{2}(X)$ by Theorem 2.4.10.

\subsection{Example}

We give an example of a two-dimensional periodic manifold which can be conformally deformed in such a way that spectral gaps occur. Let $M^{\text {per }}:=\mathbb{R} \times \mathbb{S}^{1}$ be a cylinder with $\Gamma=\mathbb{Z}$ acting on $M^{\text {per }}$ by $\gamma \cdot(x, \sigma)=(\gamma+x, \sigma)$. The periodic metric is given by $g^{\text {per }}=\mathrm{d} x^{2}+r^{2} \mathrm{~d} \sigma^{2}$ for some fixed $r>0$. We choose $M=[0,1] \times \mathbb{S}^{1}$ as period cell.

Let $0<a<b<1$ and let $X=[a, b] \times \mathbb{S}^{1}$ be the undisturbed region of $M$. Note that normal coordinates exist on $\overline{M \backslash X}$ (see Section 6.1). (see Figure 7.1). Suppose that the conformal factors $\rho_{\varepsilon}(\varepsilon>0)$ are monotonically decreasing as $\varepsilon \searrow 0$, and that $\rho_{\varepsilon}$ satisfy Conditions (6.4) and (6.5).

Let $\theta \in \hat{\Gamma}$. In this context we prefer to view $\theta$ as $\mathrm{e}^{\mathrm{i} \theta} \in \mathbb{S}^{1}$. Furthermore we identify functions $w(\sigma)$ on $\sigma \in \mathbb{S}^{1}$ with $2 \pi$-periodic functions on $\mathbb{R}$ which will be denoted again by $w(\sigma)$.

We first calculate the $\theta$-periodic harmonic extension $h=\Phi^{\theta} u$ of a function $u \in C^{\infty}(X)$ given by $u(x, \sigma)=v(x) \mathrm{e}^{\mathrm{i} n \sigma}$ for some $n \in \mathbb{Z}$, i.e., we have to solve the boundary value problem

$$
\begin{aligned}
& h(a, \cdot)=u(a, \cdot) \\
& \Delta_{M \backslash X} h=-\partial_{x x} h-\frac{1}{r^{2}} \partial_{\sigma \sigma} h=0, \quad \text { with } \quad \begin{array}{l}
h(b, \cdot)=u(b, \cdot) \\
h(1, \cdot)=\mathrm{e}^{i \theta} h(0, \cdot)
\end{array} \\
& \partial_{x} h(1, \cdot)=\mathrm{e}^{i \theta} \partial_{x} h(0, \cdot)
\end{aligned}
$$

which has a unique solution by Theorem 3.5.3. Separating the variables $h(x, \sigma)=f(x) g(\sigma)$ yields the two ODEs

$$
f^{\prime \prime}(x)=c f(x) \quad \text { and } \quad g^{\prime \prime}(\sigma)=-c r^{2} g(\sigma)
$$


Since $g$ has period $2 \pi$ we have $c=n^{2} / r^{2}$ with $n \in \mathbb{Z}$ and $g(\sigma)=\mathrm{e}^{ \pm \mathrm{i} n \sigma}$. Furthermore we have two fundamental solutions for the ODE in $f$ given by

$$
f_{1}(x)=\left\{\begin{array}{ll}
\mathrm{e}^{n x / r} & \text { if } n \neq 0, \\
x & \text { if } n=0
\end{array} \text { and } \quad f_{2}(x)= \begin{cases}\mathrm{e}^{-n x / r} & \text { if } n \neq 0 \\
1 & \text { if } n=0\end{cases}\right.
$$

Since $x$ varies over two disjoint intervals we have four independent constants in the general solution:

$$
f(x)= \begin{cases}c_{1} f_{1}(x)+c_{2} f_{2}(x) & \text { if } 0 \leq x \leq a, \\ d_{1} f_{1}(x)+d_{2} f_{2}(x) & \text { if } b \leq x \leq 1\end{cases}
$$

The boundary conditions in (7.8) now require that $g(\sigma)=\mathrm{e}^{\mathrm{i} n \sigma}$ and

$$
\begin{aligned}
f_{1}(a) c_{1}+f_{2}(a) c_{2} & =v(a) \\
& f_{1}(b) d_{1}+f_{2}(b) d_{2}=v(b) \\
\mathrm{e}^{\mathrm{i} \theta} f_{1}(0) c_{1}+\mathrm{e}^{\mathrm{i} \theta} f_{2}(0) c_{2}-f_{1}(1) d_{1}-f_{2}(1) d_{2} & =0 \\
\mathrm{e}^{\mathrm{i} \theta} f_{1}^{\prime}(0) c_{1}+\mathrm{e}^{\mathrm{i} \theta} f_{2}^{\prime}(0) c_{2}-f_{1}^{\prime}(1) d_{1}-f_{2}^{\prime}(1) d_{2} & =0 .
\end{aligned}
$$

We obtain the solution

$$
\begin{aligned}
& c_{1}=\gamma\left(+\mathrm{e}^{(1-b) \beta} v(a)-\mathrm{e}^{-\mathrm{i} \theta-a \beta} v(b)\right) \\
& c_{2}=\gamma\left(-\mathrm{e}^{-(1-b) \beta} v(a)+\mathrm{e}^{-\mathrm{i} \theta+a \beta} v(b)\right) \\
& d_{1}=\gamma\left(+\mathrm{e}^{\mathrm{i} \theta-b \beta} v(a)-\mathrm{e}^{-(1+a) \beta} v(b)\right) \\
& d_{2}=\gamma\left(-\mathrm{e}^{\mathrm{i} \theta+b \beta} v(a)+\mathrm{e}^{(1+a) \beta} v(b)\right)
\end{aligned}
$$

if $n \neq 0$ where we have set

$$
\begin{array}{llrl}
L & =b-a>0, & l & =1-L>0, \\
\beta & =\frac{n}{r} \quad \text { and } & \gamma & =\frac{1}{\mathrm{e}^{l \beta}-\mathrm{e}^{-l \beta}}=\frac{1}{2 \sinh (l \beta)},
\end{array}
$$

resp.

$$
\begin{aligned}
& c_{1}=\frac{1}{l}\left(v(a)-\mathrm{e}^{-\mathrm{i} \theta} v(b)\right) \\
& c_{2}=v(a)-\frac{a}{l}\left(v(a)-\mathrm{e}^{-\mathrm{i} \theta} v(b)\right) \\
& d_{1}=\frac{1}{l}\left(\mathrm{e}^{\mathrm{i} \theta} v(a)-v(b)\right) \\
& d_{2}=v(b)-\frac{b}{l}\left(\mathrm{e}^{\mathrm{i} \theta} v(a)-v(b)\right)
\end{aligned}
$$

for $n=0$. Note that $c_{i}=c_{i}(v)$ and $d_{i}=d_{i}(v)$ depend lineary on $v$.

Now we search for eigenvalues $\lambda=\lambda_{k}\left(q_{0}^{\theta}\right) \geq 0$ and eigenfunctions $u=u^{\theta} \neq 0$ of $Q_{0}^{\theta}$. By Lemma 7.2.3 $u$ satisfies

$$
\Delta_{X} u=-\partial_{x x} u-\frac{1}{r^{2}} \partial_{\sigma \sigma} u=\lambda u
$$

Again, by separating the variables $u(x, \sigma)=v(x) w(\sigma)$ we obtain the formal solutions

$$
v(x)=A_{1} v_{1}(x)+A_{2} v_{2}(x) \quad \text { and } \quad w(\sigma)=\mathrm{e}^{\mathrm{i} n \sigma}
$$


with $A_{1}, A_{2} \in \mathbb{R}$ where $v_{1}, v_{2}$ are fundamental solutions of $-v^{\prime \prime}=\mu v$, i.e.,

$$
v_{1}(x)=\left\{\begin{array}{ll}
\mathrm{e}^{\mathrm{i} \omega x} & \text { if } \mu>0, \\
x & \text { if } \mu=0, \\
\mathrm{e}^{\omega x} & \text { if } \mu<0
\end{array} \text { and } \quad v_{2}(x)= \begin{cases}\mathrm{e}^{-\mathrm{i} \omega x} & \text { if } \mu>0, \\
1 & \text { if } \mu=0, \\
\mathrm{e}^{-\omega x} & \text { if } \mu<0\end{cases}\right.
$$

with $\omega=\sqrt{|\mu|}$. Therefore the eigenvalue of the full problem is given by $\lambda=\mu+n^{2} / r^{2} \geq 0$ for $n \in \mathbb{Z}$ and $\mu \geq-n^{2} / r^{2}$. The eigenfunction lies in the domain of $Q_{0}^{\theta}$, i.e., the normal derivatives of $u$ and $h$ agree on $\partial X$. Therefore we require

$$
v^{\prime}(a)=f^{\prime}(a) \quad \text { and } \quad v^{\prime}(b)=f^{\prime}(b) .
$$

This becomes a linear system for the unknown $A_{1}$ and $A_{2}$ with the coefficient matrix

$$
A=\left(\begin{array}{cc}
v_{1}^{\prime}(a)-c_{1}\left(v_{1}\right) f_{1}^{\prime}(a)-c_{2}\left(v_{1}\right) f_{2}^{\prime}(a) & v_{2}^{\prime}(a)-c_{1}\left(v_{2}\right) f_{1}^{\prime}(a)-c_{2}\left(v_{2}\right) f_{2}^{\prime}(a) \\
v_{1}^{\prime}(b)-d_{1}\left(v_{1}\right) f_{1}^{\prime}(b)-d_{2}\left(v_{1}\right) f_{2}^{\prime}(b) & v_{2}^{\prime}(b)-d_{1}\left(v_{2}\right) f_{1}^{\prime}(b)-d_{2}\left(v_{2}\right) f_{2}^{\prime}(b)
\end{array}\right) .
$$

A non-trivial solution $v$ exists if the determinant of $A$ vanishes. This condition determines our possible values of $\lambda$ in dependence upon $\theta$ (and $n$ ).

We have to distinguish five cases:

Case $A: n=0$ and $\mu=0$. A simple calculation shows that

$$
\operatorname{det} A=\frac{2}{l}(1-\cos \theta)
$$

The only solution of $\operatorname{det} A=0$ is $\theta=0$. The corresponding eigenfunction is the constant function (i.e., $A_{1}=0$ ).

Case $B: n=0$ and $\mu>0$. A longer but straightforward calculation yields

$$
\operatorname{det} A=2 i \omega\left(\frac{2}{l}(\cos (L \omega)-\cos \theta)-\omega \sin (L \omega)\right) .
$$

The solutions of $\operatorname{det} A=0$ are analysed later on.

Case $C: n \neq 0$ and $-n^{2} / r^{2} \leq \mu=-\omega^{2}<0$ i.e., $\beta^{2} \geq \omega^{2}>0$. We obtain

$$
\operatorname{det} A=2\left(\left(\beta^{2}+\omega^{2}\right) \sinh (L \omega)+2 \beta \omega \frac{\cosh (l \beta) \cosh (L \omega)-\cos \theta}{\sinh (l \beta)}\right) .
$$

Here we have no solution since $\omega>0$ and therefore $\operatorname{det} A>0$.

Case $D: n \neq 0$ and $\mu=0$. Here, we have

$$
\operatorname{det} A=L \beta^{2}+2 \beta \frac{\cosh (l \beta)-\cos \theta}{\sinh (l \beta)}>0
$$

since $\beta \neq 0$. Again, we have no solution.

Case $E: n \neq 0$ and $\omega^{2}=\mu>0$. We obtain

$$
\operatorname{det} A=2 \mathrm{i}\left(\left(\beta^{2}-\omega^{2}\right) \sin (L \omega)+2 \beta \omega \frac{\cosh (l \beta) \cos (L \omega)-\cos \theta}{\sinh (l \beta)}\right) .
$$




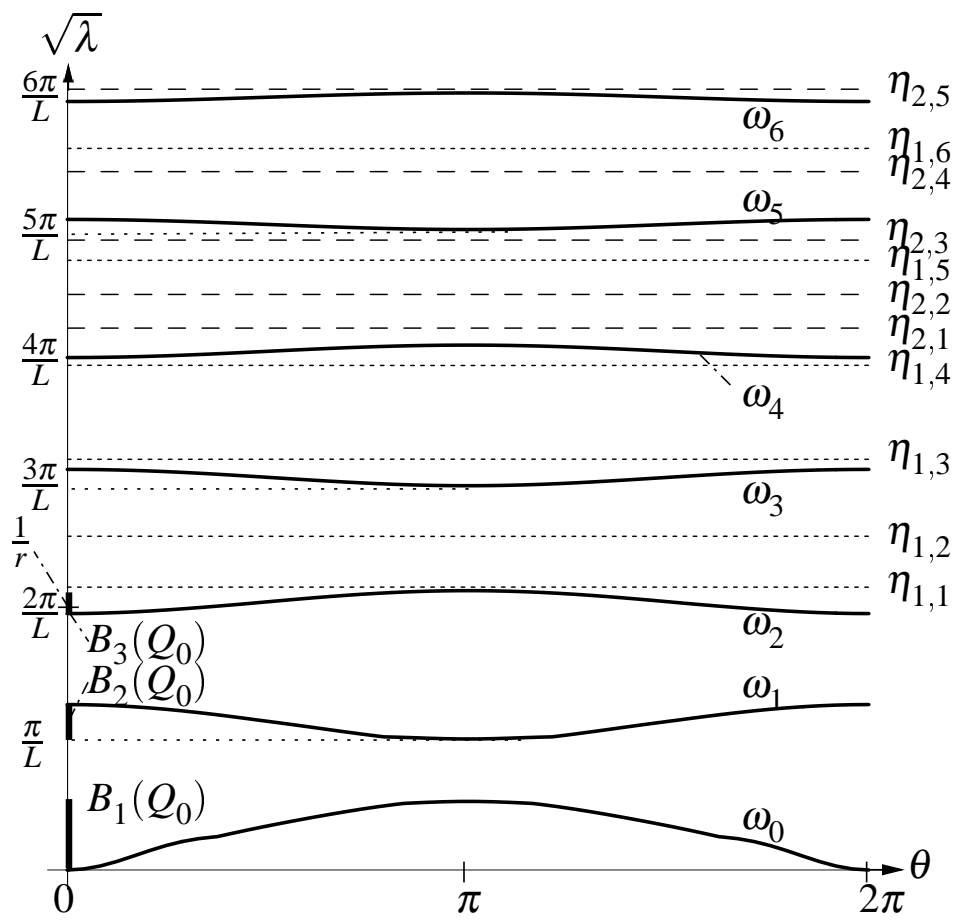

Figure 7.2: The square root of the eigenvalues of the limit operator $Q_{0}^{\theta}$ in Case A, B (thick line) and E (dotted and dashed line) plotted for $L=0.5$ and $r=1 / 13$. Here, at least $m=2$ gaps occur.

The equation $\operatorname{det} A=0$ has solutions.

From Case A and Case B we obtain smooth functions $\theta \mapsto \omega_{m}(\theta)$ for each $m \in \mathbb{N}_{0}$ solving the equation $\operatorname{det} A=0$. Note that $\omega_{m}(\theta)$ is the square root of an eigenvalue $\lambda=\lambda_{k}\left(q_{0}^{\theta}\right)$ of the limit operator (see Figure 7.2). Furthermore, note that the compact intervals $B_{m}:=$ $\left\{\omega_{m}^{2}(\theta) \mid 0 \leq \theta \leq 2 \pi\right\}$ are all disjoint: One can prove that

$$
](m \pi / L)^{2}-\varepsilon_{0},(m \pi / L)^{2}\left[\cap B_{m^{\prime}}=\emptyset\right.
$$

for all $m, m^{\prime} \in \mathbb{N}_{0}$ if $\varepsilon_{0}=\varepsilon_{0}(L)$ is small enough. Finally note that $(m \pi / L)^{2}$ are the Neumann eigenvalues of the interval $[0, L]$.

In Case $\mathrm{E}$ we denote the square root of the eigenvalue by $\eta$, i.e., $\eta=\sqrt{\lambda}=\sqrt{\omega^{2}+\beta^{2}}$. We always have

$$
\eta \geq \beta=\beta_{n}=\frac{n}{r} \geq \frac{1}{r}
$$

If we replace $\omega$ by $\sqrt{\eta^{2}-\beta_{n}^{2}}$ in (7.19) we obtain solutions $\theta \mapsto \eta_{n, p}(\theta)$ of $\operatorname{det} A=0$ for $n, p \in$ $\mathbb{N}$ (see Figure 7.2). Note that we do not expect that the intervals $B_{n, p}:=\left\{\eta_{n, p}^{2}(\theta) \mid 0 \leq \theta \leq 2 \pi\right\}$ are disjoint, we rather expect that the intervals $B_{n, p}$ cover the gaps between the intervals $B_{m}$ when $m, n$ or $p$ are large. But we still have $B_{n, p} \geq \beta_{1}=1 / r$, i.e., if $r \leq \frac{L}{m \pi}$, the intervals $B_{0}, \ldots, B_{m}$ remain disjoint. Therefore we have proven the following:

7.3.1. Theorem. Let $Q_{0}^{\theta}$ be the limit operator on the cylinder $M=[0,1] \times \mathbb{S}^{1}$ with radius $r>0$, with $X=[a, b] \times \mathbb{S}^{1}$ such that $0<a<b<1$ and with eigenvalues denoted by $\lambda_{k}\left(q_{0}^{\theta}\right)$. 
Set $L=b-a$. Then we have at least $m$ gaps between the intervals

$$
B_{k}\left(Q_{0}\right):=\left\{\lambda_{k}\left(q_{0}^{\theta}\right) \mid 0 \leq \theta \leq 2 \pi\right\}
$$

for $1 \leq k \leq m+1$, provided $r \leq \frac{L}{m \pi}$.

In particular, if we denote the conformally perturbed periodic Laplacian on $M_{\varepsilon}^{\mathrm{per}}=\mathbb{R} \times$ $\mathbb{S}^{1}$ with metric $g_{\varepsilon}^{\text {per }}=\rho_{\varepsilon}^{2}\left(\mathrm{~d} x^{2}+r^{2} \mathrm{~d} \sigma^{2}\right)$ and conformal factor $\rho_{\varepsilon}$ satisfying the conditons of Theorem 7.2.1 by $\Delta_{M_{\varepsilon}^{\mathrm{per}}}$ then the spectrum of $\Delta_{M_{\varepsilon}^{\mathrm{per}}}$ has at least $m$ gaps if $r \leq \frac{L}{m \pi}$ and if $\varepsilon>0$ is small enough.

\subsection{Mid-degree forms}

In this section we consider how the Laplacian on differential forms behaves in some special cases under conformal deformation. Again, denote by $M$ a period cell of a periodic manifold $M^{\text {per }}$. Let $X$ be a subset of $M$ with smooth boundary and with strictly positive distance from the boundary of $M$ (see Section 6.1). Suppose that $\rho_{\varepsilon}(\varepsilon>0)$ are conformal factors satisfying Conditons (6.4) and (6.5). Suppose in addition that $\rho_{\varepsilon}$ are monotonically decreasing if $\varepsilon \searrow 0$.

First, we note that for mid-degree forms, i.e., $p$-forms on a manifold of dimension $d=2 p$, we have

$$
\|u\|_{L_{2}\left(\mathbf{A}^{p} T M_{\varepsilon}\right)}=\|u\|_{L_{2}\left(\mathbf{A}^{p} T M\right)}
$$

by (6.1), i.e., the norm is independent of the conformal factor $\rho_{\varepsilon}$. By the assumptions made on $\rho_{\varepsilon}$, the quadratic form

$$
q_{\mathbf{A}^{p} T M_{\varepsilon}}(u)=\int_{M}\left(|\mathrm{~d} u|^{2}+\left|\mathrm{d}^{*} u\right|^{2}\right) \rho_{\varepsilon}^{-2}
$$

is monotonically increasing for $u \in \mathscr{H}^{1}\left(\mathbf{A}^{p} T M\right)$ and converges to the quadratic form

$$
q_{0}^{\theta}(u)=\int_{X}\left(|\mathrm{~d} u|^{2}+\left|\mathrm{d}^{*} u\right|^{2}\right)
$$

for all

$$
u \in \operatorname{dom} q_{0}^{\theta}=\left\{u \in \mathscr{H}_{\theta}^{1}\left(\mathbf{A}^{p} T M\right) \mid \mathrm{d} u \uparrow_{M \backslash X}=0, \quad \mathrm{~d}^{*} u \uparrow_{M \backslash X}=0\right\} .
$$

By the monotone convergence of quadratic forms (see [RS80, Theorem S.14]), the operator $\Delta_{\mathbf{A}^{p} T M_{\varepsilon}}^{\theta}$ converges to $Q_{0}^{\theta}$ in strong resolvent sense, i.e.,

$$
\left(\Delta_{\mathbf{A}^{p} T M_{\varepsilon}}^{\theta}+1\right)^{-1} u \rightarrow\left(\left(Q_{0}^{\theta}+1\right)^{-1} \oplus 0\right) u
$$

as $\varepsilon \rightarrow 0$ for all $u \in L_{2}\left(\mathbf{A}^{p} T M\right)$. Here, $Q_{0}^{\theta}$ denotes the operator corresponding to the form $q_{0}^{\theta}$ in $L_{2}\left(\mathbf{A}^{p} T X\right)$ and the direct sum refers to the splitting into $L_{2}$-sections on $X$ and $M \backslash X$. Since the spectrum of $\Delta_{\mathbf{A}^{p} T M_{\varepsilon}}^{\theta}$ is discrete we conclude from Theorem VII.24 of [RS80] that the $\lambda_{k}^{\theta}\left(\mathbf{A}^{p} T M_{\varepsilon}\right)$ converge to some point $\lambda \in \operatorname{spec} Q_{0}^{\theta}$ as $\varepsilon \rightarrow 0$ for every $k \in \mathbb{N}$. But we do not know anything about the limit operator $Q_{0}^{\theta}$. Note that not all elements $u \in \mathscr{H}^{1}\left(\mathbf{A}^{p} T X\right)$ can be 
extended to an element $\widetilde{u} \in \operatorname{dom} q_{0}^{\theta}$. Furthermore, the boundary condition satisfied by elements of $\operatorname{dom} q_{0}^{\theta}$ still depends on the behaviour of the "harmonic" extension $\widetilde{u}$ on $M \backslash X$.

In dimension 2, it is much easier to apply Theorem 3.4.8: the spectrum of $\Delta_{\mathbf{A}^{p} T M_{\varepsilon}^{\text {per }}}$ is the same for $p=0, p=1$ and $p=2$. Therefore, if $\Delta_{M_{\varepsilon}^{\text {per }}}$ has a gap in the essential spectrum, then this is true for $\Delta_{\mathbf{A}^{1} T M_{\varepsilon}^{\text {per }}}$ and $\Delta_{\mathbf{A}^{2} T M_{\varepsilon}^{\text {per }}}$. 


\section{Eigenvalues in spectral gaps}

In the sequel, we follow the ideas of [AADH94] and [HB00]. Let $M^{\text {per }}$ be one of the periodic manifolds with gaps in the spectrum constructed in the previous chapters. We first analyse an approximating problem, i.e., we consider the Dirichlet Laplacian on a compact subset $M^{n} \nearrow M^{\text {per }}$. Then the spectrum is purely discrete and we can count eigenvalues arising from a perturbation on a fixed subset $M^{n_{0}}$ of $M^{n}$. The main difficulty is to prove that eigenfunctions corresponding to a fixed eigenvalue $\lambda$ in a gap converge to eigenfunctions of the whole problem as $n \rightarrow \infty$.

Note that the Dirichlet boundary condition on $M^{n}$ produces no eigenvalues in the gap $] a, b[$ since the boundary of $M^{n}$ is small in some sense. This fact simplifies our proof and we do not need the more complicated construction in [AADH94].

Furthermore, note that by Theorem 2.3.8 the following results on eigenvalues in gaps of the Laplacian on functions remain true for the Laplacian on $d$-forms where $d$ denotes the dimension of the manifold. Furthermore, if $d=2$ then we can even prove the existence of eigenvalues for the Laplacian on 1- and 2-forms by Corollary 2.3.10.

\subsection{Approximating problem}

Here we consider one of the $\Gamma$-periodic manifolds $M_{\varepsilon}^{\text {per }}$ resp. $\widetilde{M}_{\varepsilon}^{\text {per }}$ constructed in Chapters 4, 5, 6 and 7. To avoid problems arising from regularity theory we assume that there exists a period cell $M_{\varepsilon}$ of $M_{\varepsilon}^{\text {per }}$ with smooth boundary, i.e., $\partial M_{\varepsilon}$ splits into $2 r$ disjoint smooth manifolds $Z^{i}$ if $r$ denotes the number of generators of $\Gamma$ (see Figure 8.1). Hence, we exclude the cases in which $M_{\mathcal{E}}$ has singularities like corners etc.

Let $\Gamma^{\prime}$ be a subset of the group $\Gamma$. Then we set

$$
\Gamma^{\prime} M_{\varepsilon}:=\bigcup_{\gamma \in \Gamma^{\prime}} \gamma M_{\mathcal{\varepsilon}}
$$

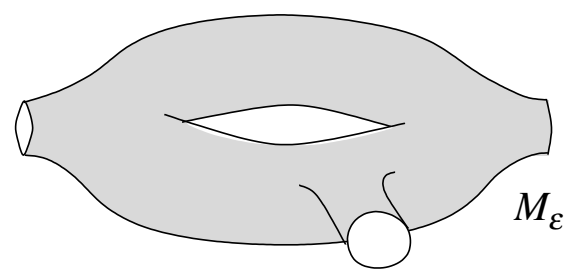

Figure 8.1: A period cell $M_{\varepsilon}$ with smooth boundary $\partial M_{\varepsilon}$. The corresponding periodic manifold $M_{\varepsilon}^{\text {per }}$ is periodic with respect to a group with $r=2$ generators. 
Note that by our smoothness assumption, $\partial \Gamma^{\prime} M_{\varepsilon}$ is also smooth.

For a subset $\Gamma^{n}$ of $\Gamma$ with $n$ elements we denote by $\Delta_{\Gamma^{n} M_{\varepsilon}}^{\mathrm{D} / \mathrm{N}}$ either the Laplacian with Dirichlet or Neumann or mixed boundary conditions on $\partial \Gamma^{n} M_{\varepsilon}$ and by $\lambda_{k}^{\mathrm{D} / \mathrm{N}}\left(\Gamma^{n} M_{\varepsilon}\right)$ its eigenvalues.

The following theorem and its corollary ensure that the spectrum of $\Delta_{M_{\varepsilon}^{\mathrm{per}}}$ and $\Delta_{\Gamma^{n} M_{\varepsilon}}^{\mathrm{D} / \mathrm{N}}$ have common gaps:

8.1.1. Theorem. Let $k \in \mathbb{N}$. Suppose that $\lambda_{k}^{\mathrm{N}}\left(M_{\mathcal{\varepsilon}}\right) \rightarrow \underline{\lambda}_{k}$ and $\lambda_{k}^{\mathrm{D}}\left(M_{\varepsilon}\right) \rightarrow \bar{\lambda}_{k}$ as $\varepsilon \rightarrow 0$ for some positive numbers $0 \leq \underline{\lambda}_{k} \leq \bar{\lambda}_{k}$.

Then both $\lambda_{k}^{\theta}\left(M_{\varepsilon}\right)$ and $\lambda_{m}^{\mathrm{D} / \mathrm{N}}\left(\Gamma^{n} M_{\varepsilon}\right)$ have the same error estimates for all $m=(k-1) n+$ $1, \ldots, k n$, i.e., there exist positive functions $\underline{\delta}_{k}(\varepsilon)$ and $\bar{\delta}_{k}(\varepsilon)$ converging to 0 as $\varepsilon \rightarrow 0$ such that

$$
\underline{\lambda}_{k}-\underline{\delta}_{k}(\varepsilon) \leq \lambda_{k}^{\theta}\left(M_{\varepsilon}\right), \lambda_{m}^{\mathrm{D} / \mathrm{N}}\left(\Gamma^{n} M_{\varepsilon}\right) \leq \bar{\lambda}_{k}+\bar{\delta}_{k}(\varepsilon) \text {. }
$$

Proof. Denote by $\Sigma^{n} M_{\varepsilon}$ the disjoint union of $n$ copies of $M_{\varepsilon}$. Then $\lambda_{m}^{\mathrm{D}}\left(\sum^{n} M_{\varepsilon}\right)=\lambda_{k}^{\mathrm{D}}\left(M_{\varepsilon}\right)$ and $\lambda_{m}^{\mathrm{N}}\left(\Sigma^{n} M_{\varepsilon}\right)=\lambda_{k}^{\mathrm{N}}\left(M_{\varepsilon}\right)$ for all $m=(k-1) n+1, \ldots, k n$ since every eigenvalue on the disjoint union has multiplicity $j n$ if the corresponding eigenvalue on $M_{\mathcal{E}}$ has multiplicity $j$. By Dirichlet-Neumann bracketing (see (2.16)) we obtain

$$
\lambda_{k}^{\mathrm{N}}\left(M_{\varepsilon}\right)=\lambda_{m}^{\mathrm{N}}\left(\Sigma^{n} M_{\varepsilon}\right) \leq \lambda_{m}^{\mathrm{D} / \mathrm{N}}\left(\Gamma^{n} M_{\varepsilon}\right) \leq \lambda_{m}^{\mathrm{D}}\left(\Sigma^{n} M_{\varepsilon}\right)=\lambda_{k}^{\mathrm{D}}\left(M_{\varepsilon}\right)
$$

while the Dirichlet-Neumann enclosure (see (3.12)) implies

$$
\lambda_{k}^{\mathrm{N}}\left(M_{\varepsilon}\right) \leq \lambda_{k}^{\theta}\left(M_{\varepsilon}\right) \leq \lambda_{k}^{\mathrm{D}}\left(M_{\varepsilon}\right)
$$

Thus the assumption on $\lambda_{k}^{\mathrm{D}}\left(M_{\varepsilon}\right)$ and $\lambda_{k}^{\mathrm{N}}\left(M_{\varepsilon}\right)$ implies the existence of $\underline{\delta}_{k}(\varepsilon)$ and $\bar{\delta}_{k}(\varepsilon)$.

8.1.2. Corollary. If $\bar{\lambda}_{k}<\underline{\lambda}_{k+1}$ then there exist numbers $a, b$ independent of $n$ such that $\bar{\lambda}_{k}<$ $a<b<\underline{\lambda}_{k+1}$, and the interval $\left.I:=\right] a, b[$ is a common gap in the spectrum of the Laplacians, provided $\varepsilon$ is small enough, i.e.,

$$
I \cap \operatorname{spec} \Delta_{M_{\varepsilon}^{\mathrm{per}}}=\emptyset \quad \text { and } \quad I \cap \operatorname{spec} \Delta_{\Gamma^{n} M_{\varepsilon}}^{\mathrm{D} / \mathrm{N}}=\emptyset .
$$

The next corollary will be used in Section 8.2. For the definition of the eigenvalue counting function $\operatorname{dim}_{\lambda}\left(\Delta_{\Gamma^{n} M_{\varepsilon}}^{\mathrm{D} / \mathrm{N}}\right)$ see Definition 1.3.6.

8.1.3. Corollary. Suppose that the assumptions of the previous corollary are fulfilled. If $\lambda \in I$ lies in the gap between the $k$-th and the $(k+1)$-st band, then $\operatorname{dim}_{\lambda}\left(\Delta_{\Gamma^{n} M_{\varepsilon}}^{\mathrm{D} / \mathrm{N}}\right)=k n$ independently of the boundary conditions on $\partial \Gamma^{n} M_{\mathcal{\varepsilon}}$.

For the rest of this chapter, we fix $k \in \mathbb{N}$ such that $\bar{\lambda}_{k}<a<b<\underline{\lambda}_{k+1}$ and $\left.\lambda \in I=\right] a, b[$. Furthermore, we fix $\varepsilon>0$ such that $I$ is a common gap as in Corollary 8.1.2 and therefore omit $\varepsilon$ in the notation, e.g., $M=M_{\varepsilon}$.

Let $\Gamma^{n} \nearrow \Gamma$ be an exhaustive sequence, i.e., a monotone sequence with $\bigcup_{n} \Gamma^{n}=\Gamma$. Suppose further that $M^{n}:=\Gamma^{n} M, R^{n}:=\left(\Gamma \backslash \Gamma^{n}\right) M$ and $R^{n_{0}, n}:=\left(\Gamma^{n} \backslash \Gamma^{n_{0}}\right) M$ for $n_{0}<n$ are all connected (see Figure 8.2). Note that $M^{n} \nearrow M^{\text {per }}$ and $R^{n_{0}, n} \nearrow R^{n_{0}}$ as $n \rightarrow \infty$ and that $M^{\text {per }}=M^{n} \cup R^{n}$ and $M^{n}=M^{n_{0}} \cup R^{n_{0}, n}$. 


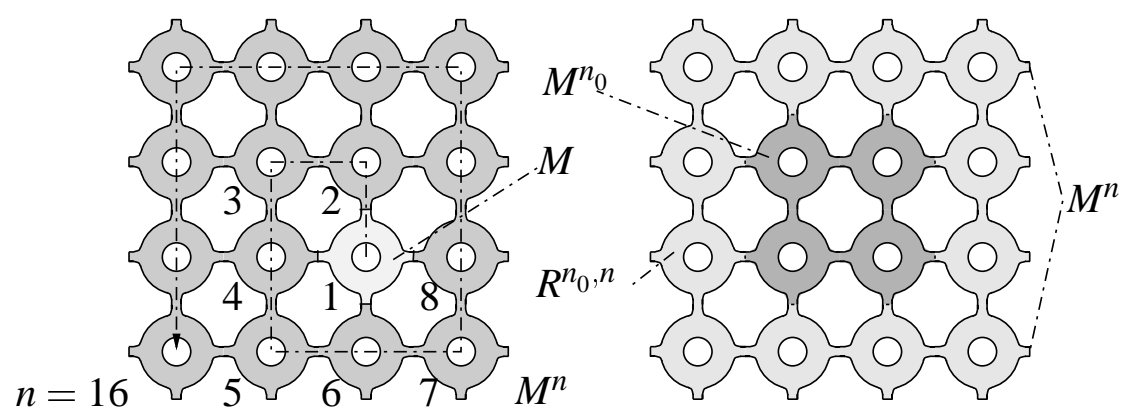

Figure 8.2: The approximating manifold $M^{n}$ on the right hand side and the manifolds $M^{n_{0}}, M^{n}$ and $R^{n_{0}, n}$ on the left hand side $\left(n_{0}=4\right.$ and $\left.n=16\right)$.

Now we consider perturbations of the periodic metric $g^{\text {per }}$ on $M^{\text {per }}$. Let $(g(\tau))_{\tau}$ be a family of Riemannian metrics on $M^{\text {per }}$. The Riemannian manifold $\left(M^{\text {per }}, g(\tau)\right)$ will be written briefly as $M(\tau)$. It is quite obvious how to understand notations like $M^{n}(\tau), R^{n}(\tau)$ and so on.

Now we make our assumptions on the family of metrics $(g(\tau))_{\tau}$ :

$$
\begin{aligned}
& g(0)=g^{\text {per }} \\
& \left\|g(\tau)-g\left(\tau_{0}\right)\right\|_{C^{1}\left(M^{\text {per }}\right)} \rightarrow 0 \quad \text { as } \quad \tau \rightarrow \tau_{0} \\
& \sup _{\tau>0}\|g(\tau)-g(0)\|_{C^{1}\left(R^{n} 0\right)} \rightarrow 0 \quad \text { as } \quad n_{0} \rightarrow \infty
\end{aligned}
$$

for all $\tau_{0} \geq 0$.

The first assumption assures that we start with the periodic manifold. The second one is a kind of continuity and the third one tells us, roughly speaking, that outside of a bounded region there is more or less no perturbation.

Next we need some arguments used in Theorem 8.1.6 to prove that the limits of the eigenfunctions are still lineary independent.

8.1.4. Lemma. Let $\tau_{0}>0$. Then there exists $c=c\left(\tau_{0}\right)>1$ such that

$$
\frac{1}{c}\|u\|_{L_{2}\left(M^{n}\left(\tau^{\prime}\right)\right)} \leq\|u\|_{L_{2}\left(M^{n}(\tau)\right)} \leq c\|u\|_{L_{2}\left(M^{n}\left(\tau^{\prime}\right)\right)}
$$

for all $u \in L_{2}\left(M^{n}\right)$ and $n \in \mathbb{N}$ and $0 \leq \tau, \tau^{\prime} \leq \tau_{0}$.

Proof. We apply Lemma 2.4.6 twice with $g=g(\tau)$ and $\widetilde{g}=g\left(\tau^{\prime}\right)$ and vice versa. By Assumption (8.2), $g(\tau)$ depends continuously on $\tau$. Therefore there exists $c^{\prime}>0$ such that $\eta\left(\left\|g(\tau)-g\left(\tau^{\prime}\right)\right\|_{C^{1}\left(M^{n}\right)}\right) \leq c^{\prime}$ for all $0 \leq \tau, \tau^{\prime} \leq \tau_{0}$ and $n \in \mathbb{N}$. Estimate (8.4) now follows from (2.24) with $c:=\left(1+c^{\prime}\right)^{1 / 2}$.

Let $n_{+}(n)$ be the smallest integer such that $M^{n} \subset \stackrel{\circ}{ }^{n_{+}}(n)$. Similarly, let $n_{-}(n)$ be the greatest integer such that $M^{n_{-}(n)} \subset \stackrel{\circ}{M}^{n}$ (see Figure 8.3). Note that $n_{-}(n)<n<n_{+}(n)$ and $n_{-}(n) \rightarrow \infty$ as $n \rightarrow \infty$.

8.1.5. Lemma. Let $n_{+}:=n_{+}\left(n_{0}\right)$ and $n_{-}:=n_{-}\left(n_{0}\right)$. Suppose that $\tau_{n} \rightarrow \tau$. Then there exists $c>0$ such that

$$
\left\|\Delta_{M^{n}}^{\mathrm{D}} u_{n}\right\|_{L_{2}\left(M^{n} 0\right)}^{2} \leq c^{2} \cdot\left(\left\|u_{n}\right\|_{L_{2}\left(M^{n}+(\tau)\right)}^{2}+\left\|\Delta_{M^{n}\left(\tau_{n}\right)}^{\mathrm{D}} u_{n}\right\|_{L_{2}\left(M^{n}+(\tau)\right)}^{2}\right)
$$




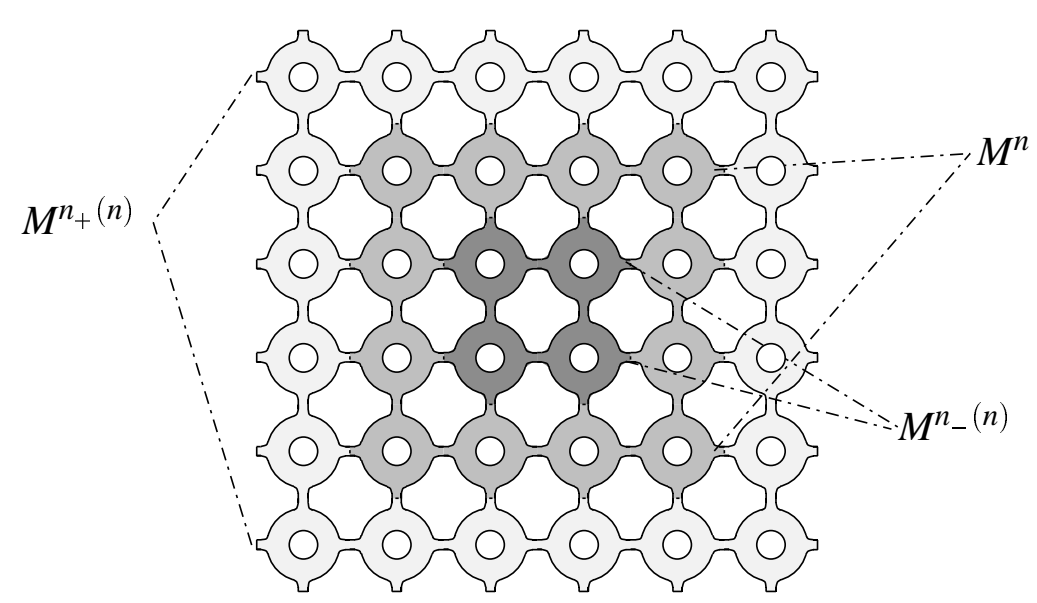

Figure 8.3: Definition of the integers $n_{+}(n)$ and $n_{-}(n)$.

for all $n>n_{++}\left(n_{0}\right):=n_{+}\left(n_{+}\left(n_{0}\right)\right)$ and all $u_{n} \in \mathscr{H}^{2}\left(M^{n}\right) \cap \dot{\mathscr{H}}^{1}\left(M^{n}\right)$. Furthermore, there exists $\delta_{n} \rightarrow 0$ as $n \rightarrow \infty$ such that

$$
\left\|\left(\Delta_{M^{n}}^{\mathrm{D}}-\Delta_{M^{n}\left(\tau_{n}\right)}^{\mathrm{D}}\right) u_{n}\right\|_{L_{2}\left(R^{n_{0}, n}\right)}^{2} \leq \delta_{n_{-}}^{2} \cdot\left(\left\|u_{n}\right\|_{L_{2}\left(M^{n}\left(\tau_{n}\right)\right)}^{2}+\left\|\Delta_{M^{n}\left(\tau_{n}\right)}^{\mathrm{D}} u_{n}\right\|_{L_{2}\left(M^{n}\left(\tau_{n}\right)\right)}^{2}\right)
$$

for all $n>n_{0}$ and all $u \in \mathscr{H}^{2}\left(M^{n}\right) \cap \mathscr{\mathscr { H }}^{1}\left(M^{n}\right)$.

Proof. We choose a function $\chi \in C_{c}^{\infty}\left(M^{n_{+}}\right)$such that $\chi\left\lceil_{M^{n_{0}}}=1\right.$. Applying Lemma 2.4.7, Theorem 2.4.10 and Lemma 2.4.9 we obtain

$$
\begin{aligned}
\left\|\Delta_{M^{n}}^{\mathrm{D}} u_{n}\right\|_{L_{2}\left(M^{n} 0\right)}^{2} & \leq\left\|\Delta_{M^{n}}^{\mathrm{D}} \chi u_{n}\right\|_{L_{2}\left(M^{n}+\right)}^{2} \\
& \leq c_{1} \cdot c_{3} \cdot\left(\left\|\chi u_{n}\right\|_{L_{2}\left(M^{n}+\left(\tau_{n}\right)\right)}^{2}+\left\|\Delta_{M^{n}\left(\tau_{n}\right)}^{\mathrm{D}} \chi u_{n}\right\|_{L_{2}\left(M^{n}+\left(\tau_{n}\right)\right)}^{2}\right) \\
& \leq c_{1} \cdot c_{3} \cdot\left(1+c_{2}\|\chi\|_{C^{1}\left(M^{\mathrm{per}}\right)}\right)\left(\left\|u_{n}\right\|_{L_{2}\left(M^{n}+\left(\tau_{n}\right)\right)}^{2}+\left\|\Delta_{M^{n}\left(\tau_{n}\right)}^{\mathrm{D}} u_{n}\right\|_{L_{2}\left(M^{n}+\left(\tau_{n}\right)\right)}^{2}\right) .
\end{aligned}
$$

We apply Lemma 8.1.4 to obtain an estimate in which the $L_{2}$-norm over $M(\tau)$ occurs. By the periodicity of $g^{\text {per }}$ and by Assumption (8.2) we can estimate

$$
c\left(\tau_{0}\right) c_{1}\left(\left\|g^{\mathrm{per}}\right\|_{C^{1}}\right) c_{3}\left(\left\|g\left(\tau_{n}\right)\right\|_{C^{1}}\right)\left(1+c_{2}\left(\left\|g\left(\tau_{n}\right)\right\|_{C^{1}}\right)\|\chi\|_{C^{1}}\right) \leq c^{2}
$$

for some constant $c>0$.

To prove the second estimate use $\chi \in C_{c}^{\infty}\left(R^{n_{-}}\right)$such that $\chi \uparrow_{R_{0}, n}=1$. The proof is essentially the same if we replace $c_{1}\left(\left\|g^{\text {per }}\right\|_{C^{1}}\right)$ by the expression $\eta_{1}\left(\left\|g^{\text {per }}-g\left(\tau_{n}\right)\right\|_{C^{1}}\right)$ which converges to 0 uniformly in $n$ as $n_{0} \rightarrow \infty$ by Assumption (8.3). Note that it is important here to apply regularity theory up to the boundary $\partial M^{n}$.

The following theorem now shows that the eigenfunctions of the approximating problem converge to eigenfunctions of the original problem without loss of multiplicity.

8.1.6. Theorem. Suppose that $\tau_{n} \rightarrow \tau$ and $\left.\lambda_{n, i} \rightarrow \lambda \in I=\right] a, b[$ for $i=1, \ldots, k$. Suppose further that $\varphi_{n, i} \in L_{2}\left(M^{n}\left(\tau_{n}\right)\right)=: \mathscr{H}_{n}, i=1, \ldots, k$, are orthonormal for all $n$ and

$$
\Delta_{M^{n}\left(\tau_{n}\right)}^{\mathrm{D}} \varphi_{n, i}=\lambda_{n, i} \varphi_{n, i}
$$


Then for every $i$ there exists a subsequence $\left(\varphi_{n_{m}, i}\right)_{m}$ converging weakly to $\varphi_{0, i} \in \operatorname{dom} \Delta_{M(\tau)}$ in $\mathscr{H}^{1}(M(\tau))$ and strongly in $L_{2, \mathrm{loc}}(M(\tau))$. Furthermore,

$$
\Delta_{M(\tau)} \varphi_{0, i}=\lambda \varphi_{0, i}
$$

for $i=1, \ldots, k$, and $\left(\varphi_{0, i}\right)_{i=1, \ldots, k}$ are orthogonal.

Proof. For every $i$ we apply Lemma 2.4 .6 with $g=g\left(\tau_{n}\right)$ and $\widetilde{g}=g(\tau)$. By Assumption (8.2) the conditions of Lemma 1.4.7 and therefore the conditions of Theorem 1.4.6 of Chapter 1 (with $\mathscr{H}:=L_{2}(M(\tau)), q_{n}:=q_{M^{n}\left(\tau_{n}\right)}^{\mathrm{D}}, q:=q_{M(\tau)}$ and $\mathscr{D}:=C_{c}^{\infty}\left(M^{\text {per }}\right)$ ) are satisfied. Note that we really need Dirichlet boundary conditions on $M^{n}\left(\tau_{n}\right)$ here since then $\operatorname{dom} q_{n}=\stackrel{\mathscr{H}}{ }^{1}\left(M^{n}\left(\tau_{n}\right)\right) \subset \mathscr{H}^{1}(M(\tau))=\operatorname{dom} q$.

Therefore (by passing to a subsequence) $\varphi_{n, i} \rightarrow \varphi_{0, i}$ weakly in $\mathscr{H}^{1}(M(\tau))$ and strongly in $L_{2, \text { loc }}(M(\tau))$ by the Rellich-Kondrachov compactness Theorem as $n \rightarrow \infty$ and $\varphi_{0, i} \in \operatorname{dom} \Delta_{M(\tau)}$. Furthermore, (8.8) is satisfied.

The main difficulty is to prove that $\varphi_{0, i}$ are lineary independent. Suppose that there exist $\alpha_{1}, \ldots, \alpha_{k}$, not all equal to 0 , such that $u_{n}:=\sum_{i} \alpha_{i} \varphi_{n, i}$ converges to $\sum_{i} \alpha_{i} \varphi_{0, i}=0$ in $L_{2, \text { loc }}(M(\tau))$. Since $\left(\tau_{n}\right)$ is bounded there exists $c_{1}>1$ such that

$$
\left\|u_{n}\right\|_{L_{2}\left(M^{n}\right)} \geq \frac{1}{c_{1}}\left\|u_{n}\right\|_{L_{2}\left(M^{n}\left(\tau_{n}\right)\right)}=\frac{1}{c_{1}} \sum_{i}\left|\alpha_{i}\right|^{2}=: c_{2}>0
$$

for all $n \in \mathbb{N}$ by Lemma 8.1.4. By Corollary 8.1.2, I is a spectral gap of $\Delta_{M^{n}}^{\mathrm{D}}$. The spectral theorem implies

$$
\left\|\left(\Delta_{M^{n}}^{\mathrm{D}}-\lambda\right) u_{n}\right\|_{L_{2}\left(M^{n}\right)} \geq(b-a)\left\|u_{n}\right\|_{L_{2}\left(M^{n}\right)} \geq \frac{b-a}{c_{2}}=: c_{3}>0
$$

for all $n \in \mathbb{N}$. On the other side we have

$$
\begin{aligned}
\left\|\left(\Delta_{M^{n}}^{\mathrm{D}}-\lambda\right) u_{n}\right\|_{L_{2}\left(M^{n}\right)} & \leq \\
& \leq\left\|\sum_{j=1}^{k}\left(\Delta_{M^{n}}^{\mathrm{D}}-\lambda_{n, j}\right) \alpha_{j} \varphi_{n, j}\right\|_{L_{2}\left(M^{n}\right)}+\sum_{j=1}^{k}\left|\alpha_{j}\right| \cdot\left|\lambda_{n, j}-\lambda\right| \cdot\left\|\varphi_{n, j}\right\|_{L_{2}\left(M^{n}\right)^{n}} .
\end{aligned}
$$

The last term converges to 0 since $\left\|\varphi_{n, j}\right\|_{L_{2}\left(M^{n}\right)} \leq c$ by Lemma 8.1.4. The first term can be split into an integral over $M^{n_{0}}$ and $R^{n_{0}, n}$. By using (8.6) and (8.7) we can estimate the integral over $R^{n_{0}, n}$ by

$$
\begin{aligned}
& \|\left(\Delta_{\left.M^{n}-\Delta_{M^{n}\left(\tau_{n}\right)}^{\mathrm{D}}\right) u_{n} \|_{L_{2}\left(R^{n_{0}, n}\right)}}\right. \\
& \quad \leq \delta_{n_{-}} \cdot\left(\left\|u_{n}\right\|_{L_{2}\left(M^{n}\left(\tau_{n}\right)\right)}^{2}+\left\|\Delta_{M^{n}\left(\tau_{n}\right)}^{\mathrm{D}} u_{n}\right\|_{L_{2}\left(M^{n}\left(\tau_{n}\right)\right)}^{2}\right)^{\frac{1}{2}} \\
& \quad \leq \delta_{n_{-}\left(n_{0}\right)} \cdot\left(\sum_{j=1}^{k}\left|\alpha_{j}\right|^{2}\left(1+\max _{j} \lambda_{n, j}^{2}\right)^{\frac{1}{2}}\right.
\end{aligned}
$$

tending to 0 as $n_{0} \rightarrow \infty$. The integral over $M^{n_{0}}$ can be estimated by

$$
\left\|\Delta_{M^{n}}^{\mathrm{D}} u_{n}\right\|_{L_{2}\left(M^{n} 0\right)}+\max _{j} \lambda_{n, j}\left\|u_{n}\right\|_{L_{2}\left(M^{n} 0\right)} \cdot
$$


Furthermore, applying (8.5) yields the estimate

$$
\begin{aligned}
\left\|\Delta_{M^{n}}^{\mathrm{D}} u_{n}\right\|_{L_{2}\left(M^{n} 0\right)} \leq c \cdot\left(\left\|u_{n}\right\|_{L_{2}\left(M^{n}+(\tau)\right)}^{2}+\left\|\Delta_{M^{n}\left(\tau_{n}\right)}^{\mathrm{D}} u_{n}\right\|_{L_{2}\left(M^{n}+(\tau)\right)}^{2}\right)^{\frac{1}{2}} & \\
& \leq c \cdot\left((1+\lambda)\left\|u_{n}\right\|_{L_{2}\left(M^{n}+(\tau)\right)}^{2}+\left\|\left(\Delta_{M^{n}\left(\tau_{n}\right)}^{\mathrm{D}}-\lambda\right) u_{n}\right\|_{L_{2}\left(M^{n}+(\tau)\right)}^{2}\right)^{\frac{1}{2}}
\end{aligned}
$$

for all $n>n_{++}\left(n_{0}\right)$. The first term under the square root converges to 0 since $u_{n} \rightarrow 0$ in $L_{2, \text { loc }}$. The second term can be estimated by

$$
\max _{j}\left|\lambda_{n, j}-\lambda\right|^{2}\left\|\varphi_{n, j}\right\|_{L_{2}\left(M^{n}\left(\tau_{0}\right)\right)}^{2} \sum_{j=1}^{k}\left|\alpha_{j}\right|^{2}
$$

which also converges to 0 . This contradicts (8.9).

Finally, we prove that the essential spectrum of the periodic and the perturbed Laplacian are the same. For the definition of the essential spectrum and singular sequences see Section 1.3.

8.1.7. Theorem. We have ess $\operatorname{spec} \Delta_{M^{\mathrm{per}}}=\operatorname{ess} \operatorname{spec} \Delta_{M(\tau)}$ for all $\tau>0$.

Proof. Let $\lambda \in$ ess $\operatorname{spec} \Delta_{M \text { per }}$ and let $\varepsilon>0$. By Lemma 2.4.7, Theorem 2.4.10 and Assumption (8.3) there exists $n_{0} \in \mathbb{N}$ such that

$$
\left\|\left(\Delta_{M(\tau)}-\Delta_{\left.M^{\mathrm{per}}\right)}\right) u\right\|_{L_{2}\left(R^{n_{0}}\right)}^{2} \leq \frac{\varepsilon}{c_{1}}\left(\|u\|_{L_{2}\left(R^{n} 0\right)}^{2}+\left\|\Delta_{M^{\mathrm{per}}} u\right\|_{L_{2}\left(R^{n_{0}}\right)}^{2}\right)
$$

for all $u \in \mathscr{H}^{2}\left(R^{n_{0}}\right) \cap \mathscr{\mathscr { H }}^{1}\left(R^{n_{0}}\right)$. Here, $c_{1}>0$ is some constant specified later on.

Let $\left(u_{n}\right)$ be a singular sequence for $\lambda$ and $\Delta_{M^{\text {per }}}$, i.e., $u_{n} \rightarrow 0$ weakly in $L_{2}\left(M^{\text {per }}\right),\left\|u_{n}\right\|=1$ and $\left\|\left(\Delta_{M \text { per }}-\lambda\right) u_{n}\right\| \rightarrow 0$ as $n \rightarrow \infty$. By the Decomposition Principle (Theorem 2.5.1) we can assume that $u_{n}$ has support away from $M^{n_{0}}$. By Lemma 8.1.4 there exists $c>0$ such that

$$
\left\|u_{n}\right\|_{L_{2}(M(\tau))} \geq \frac{1}{c}\left\|u_{n}\right\|_{L_{2}\left(M^{\mathrm{per}}\right)}=\frac{1}{c}>0
$$

for all $n$.

Now we want to show that $v_{n}:=u_{n} /\left\|u_{n}\right\|_{L_{2}(M(\tau))}$ is a singular sequence for $\lambda$ and $\Delta_{M(\tau)}$. Since the norm topology in $L_{2}\left(M^{\text {per }}\right)$ and $L_{2}(M(\tau))$ are the same, this is also true for the weak topology (see Lemma 1.2.1). By (8.11) we conclude that $v_{n} \rightarrow 0$ weakly in $L_{2}(M(\tau))$. Since $u_{n} \uparrow_{M^{n} 0}=0$ we have

$$
\begin{aligned}
\left\|\left(\Delta_{M(\tau)}-\lambda\right) v_{n}\right\|_{L_{2}(M(\tau))}^{2} & \leq 2 c^{4}\left(\left\|\left(\Delta_{M(\tau)}-\Delta_{M^{\mathrm{per}}}\right) u_{n}\right\|_{L_{2}\left(R^{n} 0\right)}^{2}+\left\|\left(\Delta_{M^{\mathrm{per}}}-\lambda\right) u_{n}\right\|_{L_{2}\left(R^{n}\right)}^{2}\right) \\
& \leq 2 c^{4} \frac{\varepsilon}{c_{1}}\left(\left\|u_{n}\right\|_{L_{2}\left(R^{n} 0\right)}^{2}+\left\|\Delta_{M^{\mathrm{per}}} u_{n}\right\|_{L_{2}\left(R^{n} 0\right)}^{2}\right)+2 c^{4}\left\|\left(\Delta_{M^{\mathrm{per}}}-\lambda\right) u_{n}\right\|_{L_{2}\left(R^{n} 0\right)}^{2} \\
& \leq 2 c^{4} \frac{\varepsilon}{c_{1}}(1+2 \lambda)+2 c^{4}\left(1+\frac{2 \varepsilon}{c_{1}}\right)\left\|\left(\Delta_{M^{\mathrm{per}}}-\lambda\right) u_{n}\right\|_{L_{2}\left(R^{n} 0\right)}^{2}
\end{aligned}
$$

by (8.10), Lemma 8.1 .4 and (8.11). Finally set $c_{1}:=4 c^{4}(1+2 \lambda)$. Then the first summand in the last line is $\varepsilon / 2$ and the second converges to 0 as $n \rightarrow \infty$. Therefore we have proven ess $\operatorname{spec} \Delta_{M^{\text {per }}} \subset$ ess $\operatorname{spec} \Delta_{M(\tau)}$. The opposite inclusion can be shown in the same way.

From the preceding Theorem and Corollary 8.1.2 we conclude:

8.1.8. Corollary. The interval $I=] a, b\left[\right.$ is an essential spectral gap for $\Delta_{M(\tau)}$ for all $\tau \geq 0$. 


\subsection{Eigenvalue counting functions}

Here we give a lower bound on the number of eigenvalues in the gap of the unperturbed operator. Again, we fix $k \in \mathbb{N}$ and $\lambda$ such that $\bar{\lambda}_{k}<a<\lambda<b<\underline{\lambda}_{k+1}$. Here, $\left.I:=\right] a, b[$ is a common gap as in Corollary 8.1.2.

First, we show that the boundary conditions on $R^{n_{0}, n}(\tau)$ are not important for the eigenvalue counting function.

8.2.1. Lemma. Denote by $\Delta_{R^{n_{0}, n}(\tau)}^{\mathrm{D} / \mathrm{N}}$ the Laplacian on $R^{n_{0}, n}(\tau)$ either with Dirichlet or Neumann or mixed boundary conditions. Then there exists $n_{0} \in \mathbb{N}$ such that

$$
\operatorname{dim}_{\lambda}\left(\Delta_{R^{n} n^{, n}(\tau)}^{\mathrm{D} / \mathrm{N}}\right)=k\left(n-n_{0}\right)
$$

for all $\tau \geq 0$ and $n \geq n_{0}$.

Proof. Choose $\eta>0$ so small such that

$$
\bar{\lambda}_{k}<\bar{\lambda}_{k}+8 \eta<a<b<\underline{\lambda}_{k+1}-8 \eta<\underline{\lambda}_{k+1} .
$$

We use Lemma 1.4 .5 with $\mathscr{H}=L_{2}\left(R^{n_{0}, n}(\tau)\right)$ and $\mathscr{H}^{\prime}=L_{2}\left(R^{n_{0}, n}\right)$. The assumptions of Lemma 1.4.5 are fulfilled by Lemma 2.4.6 if

$$
\eta\left(\sup _{\tau \geq 0}\|g(\tau)-g(0)\|_{C^{1}\left(R^{n} 0\right)}\right) \leq \eta \text {. }
$$

This can be achieved by Assumption (8.3), provided $n_{0}$ is large enough. Then we have

$$
\left|\lambda_{m}^{\mathrm{D} / \mathrm{N}}\left(R^{n_{0}, n}\right)-\lambda_{m}^{\mathrm{D} / \mathrm{N}}\left(R^{n_{0}, n}(\tau)\right)\right| \leq 4 \eta
$$

for all $\tau \geq 0$. Therefore $\lambda_{m}^{\mathrm{D} / \mathrm{N}}\left(R^{n_{0}, n}(\tau)\right)$ is so close to $\lambda_{m}^{\mathrm{D} / \mathrm{N}}\left(R^{n_{0}, n}\right)$ such that no eigenvalue of $\Delta_{R^{n_{0}, n}(\tau)}^{\mathrm{D} / \mathrm{N}}$ lies in $\left.I=\right] a, b[$ and the eigenvalue counting functions agree for $\lambda \in I$. The result follows by Corollary 8.1.3. Remember that $R^{n_{0}, n}$ consists of $n-n_{0}$ copies of the period cell M.

Next, we give lower bounds on the eigenvalue counting function of the approximating problem independent of $n$ :

8.2.2. Lemma. We have

$$
\begin{aligned}
& \operatorname{dim}_{\lambda}\left(\Delta_{M^{n}(\tau)}^{\mathrm{D}}\right)-\operatorname{dim}_{\lambda}\left(\Delta_{M^{n}}^{\mathrm{D}}\right) \geq \operatorname{dim}_{\lambda}\left(\Delta_{M^{n} 0(\tau)}^{\mathrm{D}}\right)-\operatorname{dim}_{\lambda}\left(\Delta_{M^{n_{0}}}^{\mathrm{D}}\right) \\
& \operatorname{dim}_{\lambda}\left(\Delta_{M^{n}}^{\mathrm{D}}\right)-\operatorname{dim}_{\lambda}\left(\Delta_{M^{n}(\tau)}^{\mathrm{D}}\right) \geq \operatorname{dim}_{\lambda}\left(\Delta_{M^{n} 0}^{\mathrm{N}}\right)-\operatorname{dim}_{\lambda}\left(\Delta_{M^{n}(\tau)}^{\mathrm{N}}\right)
\end{aligned}
$$

for all $\tau \geq 0$ and $n \geq n_{0}$.

Proof. By $\Delta_{R^{n}, n}^{\mathrm{ND}}$ we denote the Laplacian with Dirichlet boundary conditions on $\partial M^{n}$ and Neumann boundary conditions on $\partial R^{n_{0}, n} \backslash \partial M^{n}$. Furthermore, as a result of Lemma 1.3.7 we have the following estimate

$$
\begin{aligned}
\operatorname{dim}_{\lambda}\left(\Delta_{M^{n}(\tau)}^{\mathrm{D}}\right)-\operatorname{dim}_{\lambda}\left(\Delta_{M^{n}}^{\mathrm{D}}\right) & \\
& \geq \operatorname{dim}_{\lambda}\left(\Delta_{M^{n_{0}}(\tau)}^{\mathrm{D}}\right)+\operatorname{dim}_{\lambda}\left(\Delta_{R^{n_{0}, n}(\tau)}^{\mathrm{D}}\right)-\left(\operatorname{dim}_{\lambda}\left(\Delta_{M^{n_{0}}}^{\mathrm{N}}\right)+\operatorname{dim}_{\lambda}\left(\Delta_{R^{n_{0}, n}}^{\mathrm{ND}}\right)\right) \\
& =\operatorname{dim}_{\lambda}\left(\Delta_{M^{n_{0}(\tau)}}^{\mathrm{D}}\right)-k n_{0} .
\end{aligned}
$$

Here, we have used Corollary 8.1.3 and Lemma 8.2.1 to show the equality. Estimate (8.12) follows by applying Corollary 8.1 .3 once more. Estimate (8.13) can be shown similarly. 


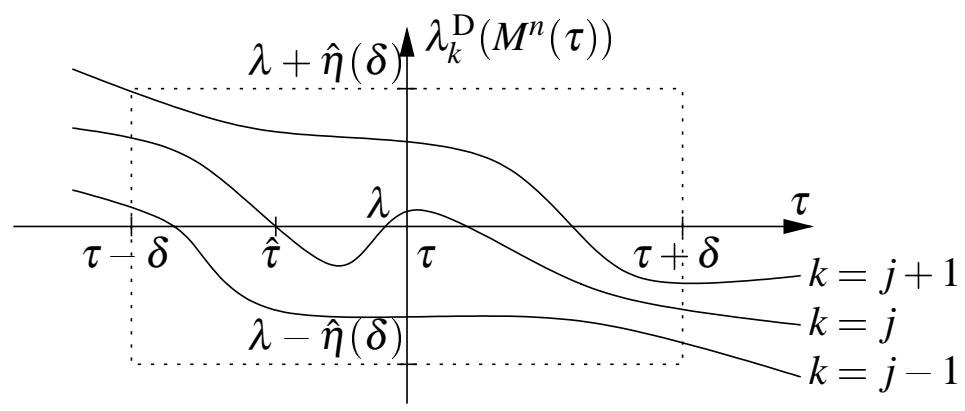

Figure 8.4: The number of eigenvalue branches $\lambda_{k}^{\mathrm{D}}\left(M^{n}(\cdot)\right)$ crossing the $\lambda$-level in $[\tau-\delta, \tau+\delta]$ can be estimated by the number of eigenvalue branches in the interval $[\lambda-\hat{\eta}(\delta), \lambda+\hat{\eta}(\delta)]$ at the fixed parameter $\tau$.

Now we show the continuity of the eigenvalue branches (for a more general result concerning the continuity of the eigenvalue branches of the Laplacian on forms see [BD97]):

8.2.3. Lemma. The function $\tau \mapsto \lambda_{k}^{\mathrm{D}}\left(M^{n_{0}}(\tau)\right)$ is continuous, in particular

$$
\left|\lambda_{m}^{\mathrm{D}}\left(M^{n_{0}}\left(\tau_{0}\right)\right)-\lambda_{m}^{\mathrm{D}}\left(M^{n_{0}}(\tau)\right)\right| \leq 4 \eta\left(\left\|g(\tau)-g\left(\tau_{0}\right)\right\|_{C^{1}\left(M^{\left.n_{0}\right)}\right.}\right) .
$$

Proof. Again, we use Lemma 1.4.5, now with $\mathscr{H}=L_{2}\left(M^{n_{0}}(\tau)\right), \mathscr{H}^{\prime}=L_{2}\left(M^{n_{0}}\left(\tau_{0}\right)\right)$ and $\eta=$ $\eta\left(\|g(\tau)-g(0)\|_{C^{1}\left(M^{n} 0\right)}\right)$.

The next result allows us to estimate the eigenvalue counting functions for different parameters $\tau$ and fixed $\lambda$ by the eigenvalue counting function for a fixed $\tau$ and an interval containing $\lambda$ (see [HB00, Section 4]). We need this lemma since we do not know whether eigenfunctions corresponding to different parameters $\tau$ are orthogonal. We do not even know that they are different! But since we want to show that the multiplicity of eigenvalues is conserved as $n \rightarrow \infty$ we need the orthogonality of the approximating eigenfunctions (see the proof of Theorem 8.2.6).

8.2.4. Lemma. For all $\tau_{0}>0$ there exists a monotone increasing function $\hat{\eta}(\delta) \rightarrow 0$ as $\delta \rightarrow 0$ such that

$$
\left|\operatorname{dim}_{\lambda}\left(\Delta_{M^{n}(\tau+\delta)}^{\mathrm{D}}\right)-\operatorname{dim}_{\lambda}\left(\Delta_{M^{n}(\tau-\delta)}^{\mathrm{D}}\right)\right| \leq \operatorname{dim}_{[\lambda-\hat{\eta}(\delta), \lambda+\hat{\eta}(\delta)]}\left(\Delta_{M^{n}(\tau)}^{\mathrm{D}}\right)
$$

for all $\lambda \geq 0, \delta>0$ and all $\tau+\delta \leq \tau_{0}$.

Proof. Since every eigenvalue branche $\lambda_{j}^{\mathrm{D}}\left(M^{n}(\cdot)\right)$ is continuous by the preceding lemma, there exists a parameter $\hat{\tau} \in[\tau-\delta, \tau+\delta]$ such that $\lambda=\lambda_{j}^{\mathrm{D}}\left(M^{n}(\hat{\tau})\right)$ by the intermediate value theorem (see Figure 8.4). Again, Lemma 8.2.3 yields

$$
\left|\lambda-\lambda_{j}^{\mathrm{D}}\left(M^{n}(\tau)\right)\right|=\left|\lambda_{j}^{\mathrm{D}}\left(M^{n}(\hat{\tau})\right)-\lambda_{j}^{\mathrm{D}}\left(M^{n}(\tau)\right)\right| \leq 4 \eta\left(\|g(\hat{\tau})-g(\tau)\|_{C^{1}\left(M^{n}\right)}\right)=: R(\tau, \hat{\tau}) .
$$

By Assumption (8.2), $R$ is uniformly continuous on $\left[0, \tau_{0}\right]^{2}$. By Lemma 2.4.3 there exists a monotone increasing function $\hat{\eta}(\delta) \rightarrow 0$ as $\delta \rightarrow 0$ such that

$$
\left|\lambda-\lambda_{j}^{\mathrm{D}}\left(M^{n}(\tau)\right)\right| \leq \hat{\eta}(|\tau-\hat{\tau}|) \leq \hat{\eta}(\delta)
$$

i.e., $\Delta_{M^{n}(\tau)}^{\mathrm{D}}$ has an eigenvalue in $[\lambda-\hat{\eta}(\delta), \lambda+\hat{\eta}(\delta)]$ 


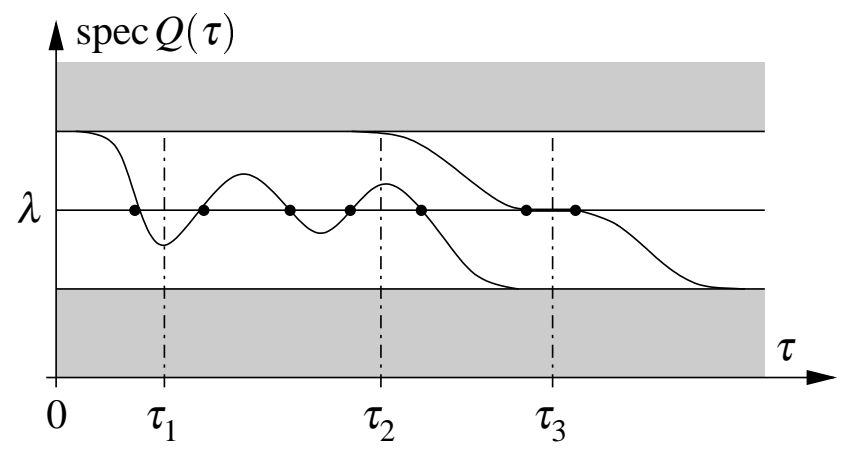

Figure 8.5: Two Eigenvalue branches in a gap of the operator $Q(0)$. Here, the eigenvalue counting function of $(Q(\tau))_{\tau}$ satisfies $\mathscr{N}_{\tau_{1}}(Q(\cdot), \lambda)=1, \mathscr{N}_{\tau_{2}}(Q(\cdot), \lambda)=4$ and $\mathscr{N}_{\tau_{3}}(Q(\cdot), \lambda)=\infty$. Note that the eigenvalue counting function does not count eigenvalue branches but the number of intersection points of the branches with the level $\lambda$.

8.2.5. Definition. For a parameter-dependent operator $Q(\tau)$ we define

$$
\mathscr{N}_{\tau}(Q(\cdot), \lambda):=\sum_{0 \leq \tau^{\prime} \leq \tau} \operatorname{dim} \operatorname{ker}\left(Q\left(\tau^{\prime}\right)-\lambda\right),
$$

the eigenvalue counting function of $(Q(\tau))_{\tau}$. The function $\mathscr{N}_{\tau}(\cdot, \lambda)$ counts the number of eigenvalues $\lambda$ (with multiplicity) of the family $(Q(\tau))_{\tau}$ (see Figure 8.5).

Note the difference to the eigenvalue counting function of a single operator $Q$ (see Definition 1.3.6), i.e.,

$$
\operatorname{dim}_{\lambda}(Q)=\sum_{0 \leq \lambda^{\prime} \leq \lambda} \operatorname{ker}\left(Q-\lambda^{\prime}\right)
$$

Finally, we prove the main result of this section (see [HB00, Section 4]):

8.2.6. Theorem. We have

$$
\mathscr{N}_{\tau}\left(\Delta_{M(\cdot)}, \lambda\right) \geq \limsup _{n \rightarrow \infty}\left|\operatorname{dim}_{\lambda}\left(\Delta_{M^{n}(\tau)}^{\mathrm{D}}\right)-\operatorname{dim}_{\lambda}\left(\Delta_{M^{n}}^{\mathrm{D}}\right)\right|
$$

Proof. Denote by

$$
T_{n}:=T_{n}(\lambda):=\left\{\tau^{\prime} \in\left[0, \tau_{0}\right] \mid \lambda \in \operatorname{spec} \Delta_{M^{n}(\tau)}^{\mathrm{D}}\right\}
$$

the set of parameters $\tau^{\prime}$ that produce an eigenvalue $\lambda$. Let $T_{\infty}$ be the set of limit points, i.e., $\hat{\tau} \in T_{\infty}$ if and only if $\hat{\tau} \in\left[0, \tau_{0}\right]$ and if there exist sequences $\left(n_{m}\right)_{m} \subset \mathbb{N}$ and $\tau_{m}^{\prime} \in T_{n_{m}}$ such that $\tau_{m}^{\prime} \rightarrow \hat{\tau}$.

We have to distinguish two cases. If the cardinality of $T_{\infty}$ is greater or equal to $N_{\lambda}$, the right hand side of (8.15), then we apply Theorem 8.1.6 with fixed eigenvalue $\lambda=\lambda_{n}=\lambda_{0}$ and with multiplicity $k=1$ for each limit point $\hat{\tau} \in T_{\infty}$. As a consequence, there are at least card $T_{\infty}$ parameters $\hat{\tau}$ such that $\lambda$ is an eigenvalue of $\Delta_{M(\hat{\tau})}$. This proves (8.15). 
If card $T_{\infty}<N_{\lambda}$ then $T_{\infty}$ consists in a finite number of points $\hat{\tau}_{1}, \ldots, \hat{\tau}_{l}$, and $T_{n} \rightarrow\left\{\hat{\tau}_{1}, \ldots, \hat{\tau}_{l}\right\}$. Furthermore, there exists a sequence $\delta_{n} \rightarrow 0$ such that

$$
\left.T_{n} \subset \bigcup_{j=1}^{k}\right] \hat{\tau}_{j}-\delta_{n}, \hat{\tau}_{j}+\delta_{n}\left[=: \hat{T}_{n}\right.
$$

for all $n \in \mathbb{N}$. If $n$ is large enough, all these intervals are mutually disjoint. As a consequence, $d_{\lambda, n}\left(\tau^{\prime}\right):=\operatorname{dim}_{\lambda}\left(\Delta_{M^{n}\left(\tau^{\prime}\right)}^{\mathrm{D}}\right)$ is constant on each component of $[0, \tau] \backslash \hat{T}_{n}$. Therefore

$$
\left|d_{\lambda, n}(\tau)-d_{\lambda, n}(0)\right| \leq \sum_{j=1}^{l}\left|d_{\lambda, n}\left(\hat{\tau}_{j}-\delta_{n}\right)-d_{\lambda, n}\left(\hat{\tau}_{j}+\delta_{n}\right)\right| .
$$

By Lemma 8.2.4 there exist $\hat{\eta}(\delta) \rightarrow 0$ as $\delta \rightarrow 0$ such that

$$
\left|d_{\lambda, n}(\tau)-d_{\lambda, n}(0)\right| \leq \sum_{j=1}^{l} \operatorname{dim}_{\left[\lambda-\hat{\eta}\left(\delta_{n}\right), \lambda+\hat{\eta}\left(\delta_{n}\right)\right]}\left(\Delta_{M^{n}\left(\hat{\tau}_{j}\right)}^{\mathrm{D}}\right) .
$$

By passing to a subsequence we can assume that $\left|d_{\lambda, n}(\tau)-d_{\lambda, n}(0)\right| \rightarrow N_{\lambda}$. We have even equality if $n$ is large enough since the sequence consists of integers. Now we select a subsequence $\left(n_{m}\right)_{m}$ such that

$$
k_{j}:=\operatorname{dim}_{\left[\lambda-\hat{\eta}\left(\delta_{n}\right), \lambda+\hat{\eta}\left(\delta_{n}\right)\right]}\left(\Delta_{M^{n}\left(\tau_{j}^{\prime}\right)}^{\mathrm{D}}\right)
$$

is independent of $m$. This is possible by choosing a convergent subsequence. Again, a converging sequence consisting of integers is constant up to finitely many exceptions. Therefore, for each $n \in \mathbb{N}$, we have $k_{j}$ orthonormal eigenfunctions $\varphi_{n, 1}, \ldots, \varphi_{n, k_{j}}$ with eigenvalues $\lambda_{n, i} \rightarrow \lambda$. Hence we can apply Theorem 8.1.6 with fixed $\hat{\tau}_{j}=\tau_{n}=\tau_{0}$ and conclude that the limit problem $\Delta_{M(\tau)}$ has $\lambda$ as eigenvalue of multiplicity $k_{j}$. This proves the theorem since $\lambda$ hat multiplicity at least $\sum_{j=1}^{l} k_{j} \geq N_{\lambda}$.

8.2.7. Remark. Note that the same result for the approximating problem is quite obvious to see: By Lemma 8.2.3, the eigenvalue branches are continuous. Therefore the eigenvalue counting function of $\Delta_{M^{n}(\cdot)}^{\mathrm{D}}$ has as a lower bound the number of eigenvalue branches passing the level $\lambda$, i.e.,

$$
\mathscr{N}_{\tau}\left(\Delta_{M^{n}(\cdot)}^{\mathrm{D}}, \lambda\right) \geq\left|\operatorname{dim}_{\lambda}\left(\Delta_{M^{n}(\tau)}^{\mathrm{D}}\right)-\operatorname{dim}_{\lambda}\left(\Delta_{M^{n}}^{\mathrm{D}}\right)\right|
$$

\subsection{Examples}

In this section we construct a perturbation of the metric such that eigenvalues in the gap of the periodic problem occur. Suppose that $I=] a, b\left[\right.$ is a common gap of $\Delta_{M^{n}}^{\mathrm{D}}$ and $\Delta_{M^{\text {per }}}$ as in Corollary 8.1.2. Finally, suppose that $\lambda \in I$. Combining Lemma 8.2.2 and Theorem 8.2.6 we obtain

$$
\begin{aligned}
& \mathscr{N}_{\tau}\left(\Delta_{M(\cdot)}, \lambda\right) \geq \operatorname{dim}_{\lambda}\left(\Delta_{M^{n_{0}(\tau)}}^{\mathrm{D}}\right)-\operatorname{dim}_{\lambda}\left(\Delta_{M^{n_{0}}}^{\mathrm{D}}\right) \\
& \mathscr{N}_{\tau}\left(\Delta_{M(\cdot)}, \lambda\right) \geq \operatorname{dim}_{\lambda}\left(\Delta_{M^{n_{0}}}^{\mathrm{N}}\right)-\operatorname{dim}_{\lambda}\left(\Delta_{M^{n_{0}}(\tau)}^{\mathrm{N}}\right) .
\end{aligned}
$$


By the Weyl asymptotics (Theorem 2.3.13) we have

$$
\operatorname{dim}_{\lambda}\left(\Delta_{M^{n} 0(\tau)}^{\mathrm{D} / \mathrm{N}}\right)-\operatorname{dim}_{\lambda}\left(\Delta_{M^{n_{0}}}^{\mathrm{D} / \mathrm{N}}\right) \approx \frac{\operatorname{vol}\left(\mathbb{B}^{d}\right)}{(2 \pi)^{d}}\left(\operatorname{vol}\left(M^{n_{0}}(\tau)\right)-\operatorname{vol}\left(M^{n_{0}}\right)\right) \lambda^{\frac{d}{2}}
$$

if $\lambda$ is large enough. This means geometrically that the number of eigenvalues in a gap has an asymptotic lower bound controlled by the volume difference of the perturbed and the unperturbed manifold.

Note that we do not know whether the counting function $\mathscr{N}_{\tau}\left(\Delta_{M(\cdot)}, \lambda\right)$ is finite or not. For example, if $g(\tau)$ is equal to a metric $g\left(\tau_{3}\right)$ for $\tau$ near $\tau_{3}$ and if $\lambda$ is an eigenvalue of $\Delta_{M\left(\tau_{3}\right)}$ then $\mathscr{N}_{\tau}\left(\Delta_{M(\cdot)}, \lambda\right)=\infty$ if $\tau \geq \tau_{3}$ (see Figure 8.5). Furthermore, the counting function $\mathscr{N}_{\tau}\left(\Delta_{M(\cdot)}, \lambda\right)$ could be much larger than the differences in the right hand side of (8.16) and (8.17): think of one eigenvalue branch passing the level $\lambda$ from above (or from below) and oszillating around $\lambda$ several times both in the approximating problem on $M^{n}(\tau)$ and in the full problem on $M(\tau)$ (again see Figure 8.5).

Finally we consider conformal deformations of the periodic metric $g^{\text {per }}$, i.e., $g(\tau)=$ $\rho^{2}(\tau) g^{\text {per }}$. Here, $\rho(\tau)$ is a smooth function on $M^{\text {per }}$ for every $\tau \geq 0$ with the following properties:

$$
\begin{aligned}
& \rho(0)=1 \\
& \left\|\rho(\tau)-\rho\left(\tau_{0}\right)\right\|_{C^{1}\left(M^{\mathrm{per}}\right)} \rightarrow 0 \quad \text { as } \quad \tau \rightarrow \tau_{0} \\
& \sup _{\tau \geq 0}\|\rho(\tau)-1\|_{C^{1}\left(R^{n} 0\right)} \rightarrow 0 \quad \text { as } \quad n_{0} \rightarrow \infty \text {. }
\end{aligned}
$$

Clearly, the family $(g(\tau))_{\tau}$ of metrics satisfies Conditions (8.1) to (8.3).

8.3.1. Eigenvalues from above. Let $K \subset \stackrel{\circ}{M}^{n_{0}}$ be a compact set with non-empty interior. Suppose further that $\rho(\tau)$ is constant on $K$ with constant $c_{\tau}$ tending to $\infty$ as $\tau \rightarrow \infty$. Then we have

$$
0 \leq \lambda_{k}^{\mathrm{D}}\left(M^{n_{0}}(\tau)\right) \leq \lambda_{k}^{\mathrm{D}}(K(\tau))=\frac{1}{c_{\tau}^{2}} \lambda_{k}^{\mathrm{D}}(K)
$$

by (2.15). Here, $K(\tau)$ denotes $K$ with metric $g(\tau)=c_{\tau}^{2} g^{\text {per }}$. Therefore, $\lambda_{k}^{\mathrm{D}}\left(M^{n_{0}}(\tau)\right) \rightarrow 0$ as $\tau \rightarrow \infty$ for all $k \in \mathbb{N}$ and $\operatorname{dim}_{\lambda}\left(\Delta_{M^{n_{0}(\tau)}}^{\mathrm{D}}\right) \rightarrow \infty$ as $\tau \rightarrow \infty$. This means that arbitrarily many eigenvalue branches cross the level $\lambda$ from above as $\tau \rightarrow \infty$.

Therefore, by Theorem 8.2.6 and Lemma 8.2.2 we have

$$
\mathscr{N}_{\tau}\left(\Delta_{M(\cdot)}, \lambda\right) \geq \operatorname{dim}_{\lambda}\left(\Delta_{M^{n} 0(\tau)}^{\mathrm{D}}\right)-\operatorname{dim}_{\lambda}\left(\Delta_{M^{n_{0}}}^{\mathrm{D}}\right) \rightarrow \infty
$$

as $\tau \rightarrow \infty$. Geometrically, we blow up the manifold in all directions with the same factor $c_{\tau}$ on $K$.

8.3.2. Eigenvalues from below. Again, let $K \subset \stackrel{\circ}{ }^{n_{0}}$ be a compact set with non-empty interior. Furthermore, let $K^{\prime}$ be the closure of $M^{n_{0}} \backslash K$. Suppose that $\rho(\tau)$ is constant on $K$ with constant $c_{\tau} \rightarrow 0$ as $\tau \rightarrow \infty$. Then we have

$$
0 \leq \lambda_{k}^{\mathrm{N}}\left(K(\tau) \dot{\cup} K^{\prime}(\tau)\right) \leq \lambda_{k}^{\mathrm{N}}\left(M^{n_{0}}(\tau)\right)
$$


by the Dirichlet-Neumann bracketing (2.16). Furthermore,

$$
\lambda_{k}^{\mathrm{N}}(K(\tau))=\frac{1}{c_{\tau}^{2}} \lambda_{k}^{\mathrm{N}}(K) \rightarrow \infty
$$

as $\tau \rightarrow \infty$ for all $k \geq 2$ and therefore $\operatorname{dim}_{\lambda}\left(\Delta_{M^{n_{0}(\tau)}}^{\mathrm{N}}\right) \rightarrow 1$ as $\tau \rightarrow \infty$ since 0 is always an eigenvalue of the Neumann problem.

By Lemma 1.3.7 we have

$$
\operatorname{dim}_{\lambda}\left(\Delta_{M^{n} 0(\tau)}^{\mathrm{N}}\right) \leq \operatorname{dim}_{\lambda}\left(\Delta_{K(\tau) \dot{\cup} K^{\prime}(\tau)}^{\mathrm{N}}\right)=\operatorname{dim}_{\lambda}\left(\Delta_{K(\tau)}^{\mathrm{N}}\right)+\operatorname{dim}_{\lambda}\left(\Delta_{K^{\prime}(\tau)}^{\mathrm{N}}\right) .
$$

Again, by Theorem 8.2.6 and Lemma 8.2.2 we conclude

$$
\mathscr{N}_{\tau}\left(\Delta_{M(\cdot)}, \lambda\right) \geq \operatorname{dim}_{\lambda}\left(\Delta_{M^{n_{0}}}^{\mathrm{N}}\right)-\operatorname{dim}_{\lambda}\left(\Delta_{M^{n_{0}(\tau)}}^{\mathrm{N}}\right) \rightarrow k n_{0}-1-\liminf _{\tau \rightarrow \infty} \operatorname{dim}_{\lambda}\left(\Delta_{K^{\prime}(\tau)}^{\mathrm{N}}\right)
$$

Here, $\lambda$ lies in the gap between the $k$-th and the $(k+1)$-st band. By the Weyl asymptotic (Theorem 2.3.13) we have

$$
\operatorname{dim}_{\lambda}\left(\Delta_{K^{\prime}(\tau)}^{\mathrm{N}}\right) \approx \frac{\operatorname{vol}\left(\mathbb{B}^{d}\right)}{(2 \pi)^{d}} \operatorname{vol}\left(K^{\prime}(\tau)\right) \lambda^{\frac{d}{2}}
$$

Now we have to choose $n_{0}, K^{\prime}$ and $\rho(\tau) \uparrow_{K^{\prime}}$ such that $\lim _{\tau \rightarrow \infty} \operatorname{vol}\left(K^{\prime}(\tau)\right)$ exists and such that the right hand side of (8.21) is strictly positive. Note that $K$ and $K^{\prime}$ also depend on $n_{0}$. If such a choice is possible, we have shown that a finite number of eigenvalues occur in the gap. Geometrically, we shrink the manifold in all directions with the same factor $c_{\tau}$ on $K$.

Note that we have not only constructed spectral gaps for the Laplacian on a periodic manifold (see Chapters 6 and 7) but also eigenvalues in a gap only by perturbing the metric conformally. 


\section{Bibliography}

[AADH94] S. Alama, M. Avellaneda, P.A. Deift, and R. Hempel, On the existence of eigenvalues of a divergence-form operator $A+\lambda B$ in a gap of $\sigma(A)$, Asymptotic Anal. 8 (1994), 311-344.

[AC93] C. Anné and B. Colbois, Opérateur de Hodge-Laplace sur des variétés compactes privées d'un nombre fini de boules, J. Funct. Anal. 115 (1993), 190-211.

[AG96] M. A. Akivis and V. V. Goldberg, Conformal differential geometry and its generalizations, John Wiley \& Sons, New York, 1996.

[Ann87] C. Anné, Spectre du Laplacien et écrasement d'anses, Ann. Sci. Éc. Norm. Super., IV. Sér. 20 (1987), 271-280.

[Ann94] C. Anné, Laplaciens en interaction, Manuscr. Math. 83 (1994), no. 1, 59-74.

[Ann99] C. Anné, Private Communication, 1999.

[BD97] G. A. Baker and J. Dodziuk, Stability of spectra of Hodge-de Rham Laplacian, Math. Zeitschrift 224 (1997), no. 3, 327-345.

[Bea83] A. F. Beardon, The geometry of discrete groups, Graduate Texts in Mathematics, Vol. 91, Springer-Verlag, New York, 1983.

[BGM71] M. Berger, P. Gauduchon, and E. Mazet, Le spectre d'une variété riemannienne, Lecture Notes in Mathematics. Vol. 194, Springer-Verlag, Berlin, 1971.

[BGV92] N. Berline, E. Getzler, and M. Vergne, Heat kernels and Dirac operators, Springer-Verlag, Berlin, 1992.

[BJR99] O. Bratteli, P. E. T. Jørgensen, and D. W. Robinson, Spectral asymptotics of periodic elliptic operators, Mathematische Zeitschrift 232 (1999), 621 - 650.

[BS92] J. Brüning and T. Sunada, On the spectrum of periodic elliptic operators, Nagoya Math. J. 126 (1992), 159-171.

[Bye70] W. P. Byers, On a theorem of Preissmann, Proc. Am. Math. Soc. 24 (1970), 50-51.

[CF78] I. Chavel and E. A. Feldman, Spectra of domains in compact manifolds, Journal of Functional Analysis 30 (1978), 198 - 222.

[CF81] I. Chavel and E.A. Feldman, Spectra of manifolds with small handles, Comment. Math. Helvetici 56 (1981), 83-102. 
[CFKS87] H.L. Cycon, R.G. Froese, W. Kirsch, and B. Simon, Schroedinger operators, with application to quantum mechanics and global geometry, Springer-Verlag, Berlin, 1987.

[Cha84] I. Chavel, Eigenvalues in Riemannian geometry, Academic Press, Orlando, 1984.

[Cha93] I. Chavel, Riemannian geometry, Cambridge University Press, Cambridge, 1993.

[Dav96] E.B. Davies, Spectral theory and differential operators, Cambridge Univerity Press, Cambridge, 1996.

[dC92] M. P. do Carmo, Riemannian geometry, Birkhäuser, Boston, 1992.

[DH87] E. B. Davies and Evans M. II Harrell, Conformally flat Riemannian metrics, Schroedinger operators, and semiclassical approximation, J. Differ. Equations 66 (1987), 165-188.

[DL79] H. Donnelly and P. Li, Pure point spectrum and negative curvature for noncompact manifolds, Duke Math. J. 46 (1979), 497-503.

[Don81] H. Donnelly, On $L^{2}$-Betti numbers for Abelian groups, Can. Math. Bull. 24 (1981), 91-95.

[DS99] E. B. Davies and Y. Safarov, Spectral theory and geometry. Proceedings of the ICMS instructional conference, Edinburgh, UK, 30 March-9 April, 1998, Cambridge Univerity Press, Cambridge, 1999.

[EŠ89] P. Exner and Šeba (eds.), Applications of self-adjoint extensions in quantum physics, Proc. Conf., Dubna/USSR 1987, Lect. Notes Phys. Vol. 324, Springer, 1989.

[Fuk87] K. Fukaya, Collapsing of Riemannian manifolds and eigenvalues of Laplace operator, Invent. Math. 87 (1987), 517-547.

[GHL87] S. Gallot, D. Hulin, and J. Lafontaine, Riemannian geometry, Springer-Verlag, Berlin, 1987.

[Gi195] P. B. Gilkey, Invariance theory, the heat equation and the Atiyah-Singer index theorem, CRC Press, Boca Raton, 1995.

[Gre97] E. L. Green, Spectral theory of Laplace-Beltrami operators with periodic metrics, J. Differ. Equations 133 (1997), no. 1, 15-29.

[Gru98] M. Gruber, Nichtkommutative Blochtheorie, Ph.D. thesis, Humboldt-Universität Berlin, 1998.

[GT77] D. Gilbarg and N. S. Trudinger, Elliptic partial differential equations of second order, Springer-Verlag, Berlin, 1977.

[HB00] R. Hempel and A. Besch, Magnetic barriers of compact support and eigenvalues in spectral gaps, Preprint (2000). 
[Heb96] E. Hebey, Sobolev spaces on Riemannian manifolds, Lecture Notes in Mathematics. Vol. 1635, Springer-Verlag, Berlin, 1996.

[Hem92] R. Hempel, Second order perturbations of divergence type operators with a spectral gap, Operator Theory: Advances and Applications 57 (1992), 117-126.

[HH95a] R. Hempel and I. Herbst, Bands and gaps for periodic magnetic Hamiltonians., Operator Theory: Advances and Applications 78 (1995), 175-184.

[HH95b] R. Hempel and I. Herbst, Strong magnetic fields, Dirichlet boundaries, and spectral gaps., Commun. Math. Phys. 169 (1995), no. 2, 237-259.

[HL99] R. Hempel and K. Lienau, Spectral properties of periodic media in the large coupling limit, To appear in Communications in Partial Differential Equations (Preprint 1999).

[Hör85] L. Hörmander, The analysis of linear partial differential operators III: Pseudodifferential operators, Springer-Verlag, Berlin, 1985.

[Jau67] W. Jaunzemis, Continuum mechanics, The MacMillan Company, New York, 1967.

[Jau72] W. Jaunzemis, Differential geometry and continuum mechanics, Tensor, n. Ser. 25 (1972), 118-124.

[Kat66] T. Kato, Perturbation theory for linear operators, Springer-Verlag, Berlin, 1966.

[LCM99] J. T. Londergan, J. P. Carini, and D. P. Murdock, Binding and scattering in two-dimensional systems. Applications to quantum wires, waveguides and photonic crystals, Lecture Notes in Physics. New Series m: Monographs. Vol. m60, Springer-Verlag, Berlin, 1999.

[Lot99] J. Lott, On the spectrum of a finite-volume negatively-curved manifold, Preprint (1999).

[Mas77] W. S. Massey, Algebraic topology: An introduction, Springer-Verlag, New York, 1977.

[Mas80] W. S. Massey, Singular homology theory, Springer-Verlag, New York, 1980.

[Pon57] L. S. Pontrjagin, Topologische Gruppen, Teubner-Verlag, Leibzig, 1957.

[Pre42] A. Preissmann, Quelques proprietes globales des espaces de Riemann, Comment. Math. Helv. 15 (1942), 175-216.

[Ros97] S. Rosenberg, The Laplacian on a Riemannian manifold, London Mathematical Society Student Texts. 31, Cambridge University Press, Cambridge, 1997.

[RS78] M. Reed and B. Simon, Methods of modern mathematical physics. IV: Analysis of operators, Academic Press, New York, 1978.

[RS80] M. Reed and B. Simon, Methods of modern mathematical physics. I: Functional analysis. Rev. and enl. ed., Academic Press, New York, 1980. 
[Rud62] W. Rudin, Fourier analysis on groups, John Wiley and Sons, New York, 1962.

[Ste70] E. M. Stein, Singular integrals and differentiability properties of functions, Princeton University Press, Princeton, 1970.

[SV98] Yu. Safarov and D. Vassiliev, The asymptotic distribution of eigenvalues of partial differential operators, Translations of Mathematical Monographs 155, Providence, 1998.

[SY94] R. Schoen and S.-T. Yau, Lectures on differential geometry, International Press, Cambridge, MA, 1994.

[Tay96] M. E. Taylor, Partial differential equations. Basic theory, Springer-Verlag, New York, 1996.

[Yos98] K. Yoshitomi, Band gap of the spectrum in periodically curved quantum waveguides, J. Diff. Equations 142 (1998), no. 1, 123-166. 


\section{Zusammenfassung}

Die vorliegende Arbeit befasst sich mit spektralen Eigenschaften des Laplace-Operators auf nichtkompakten, periodischen Mannigfaltigkeiten der Dimension $d \geq 2$. Unter einer periodischen Mannigfaltigkeit $M^{\text {per }}$ verstehen wir eine Riemannsche Mannigfaltigkeit, auf der eine abelsche Gruppe $\Gamma$ eigentlich diskontinuierlich und isometrisch operiert. Außerdem nehmen wir an, dass der Quotientenraum $M^{\text {per }} / \Gamma$ kompakt ist. Unter Zuhilfenahme der Floquet-Theorie kann man zeigen, dass das Spektrum des Laplace-Operators $\Delta_{M^{\text {per }}}$ Bandstruktur besitzt, d. h. das Spektrum ist lokal endliche Vereinigung abzählbar vieler kompakter Intervalle. Sind zwei benachbarte Intervalle disjunkt, so sprechen wir von einer Lücke im Spektrum. Unser Interesse richtet sich auf die Frage, ob es Beispiele von periodischen Mannigfaltigkeiten mit Lücken im Spektrum gibt. Im Falle von Schrödinger-Operatoren im $\mathbb{R}^{d}$ sind solche Fragen bereits vielfach untersucht worden.

Wir geben zwei Beispielklassen von periodischen Mannigfaltigkeiten an, bei denen Lücken im Spektrum entstehen. In beiden Fällen betrachten wir eine Familie von periodischen Mannigfaltigkeiten $\left(M_{\mathcal{\varepsilon}}^{\text {per }}\right)_{\mathcal{\varepsilon}}$, die in einem gewissen Sinn im Limes $\varepsilon \rightarrow 0$ entkoppeln, d. h., bei der die Verbindung zwischen den Periodenzellen im geometrischen Sinn klein wird (z. B. konvergiert das Volumen dieser Verbindung gegen 0). Unser Hauptergebnis besteht im Nachweis, dass zu jedem $N \in \mathbb{N}$ mindestens $N$ Lücken im Spektrum von $\Delta_{M_{\varepsilon}^{\text {per }}}$ in beiden Beispielklassen existieren, wenn $\varepsilon$ klein genug gewählt wird.

Abschließend stören wir die Mannigfaltigkeit lokal und zeigen die Existenz von Eigenwerten in einer solchen Lücke im Spektrum.

Der Effekt, der zur Entkopplung führt, ist hier viel subtiler und technisch schwerer zu beherrschen als bei Schrödinger-Operatoren. Da wir mit parameterabhängigen Mannigfaltigkeiten arbeiten, hängt insbesondere der zugrundeliegende Hilbertraum über die Metrik von diesem Parameter ab. Ein Teil der Arbeit widmet sich diesem Problem.

Die vorliegende Arbeit zeigt, dass bereits bekannte spektrale Aussagen über Divergenztypund Schrödinger-Operatoren auch für Laplace-(Beltrami)-Operatoren auf gewissen Mannigfaltigkeit gelten, und verbindet somit Spektraltheorie und Differentialgeometrie. 